\title{
On the quark-mass dependence of baryon ground-state masses
}

\author{
Vom Fachbereich Physik \\ der Technischen Universität Darmstadt \\ zur Erlangung des Grades \\ eines Doktors der Naturwissenschaften \\ (Dr. rer. nat.)
}

genehmigte Dissertation von Dipl.-Phys. Alexander Semke aus Nikopol (Ukraine)

Referent: PD. Dr. M. F. M. Lutz Koreferent: Prof. Dr. Ch. Fischer Tag der Einreichung: 26.01.2010 Tag der Prüfung: 17.02.2010

Darmstadt 2010

D17 



\section{Zusammenfassung}

Die vorliegende Arbeit beschäftigt sich mit der Physik der starken Wechselwirkung. Quantenchromodynamik (QCD), die allgemein anerkannte Theorie der starken Wechselwirkung, ist eine asymptotisch freie Theorie und kann nur bei hohen Energien störungstheoretisch behandelt werden. Es existieren mehrere Ansätze zur Beschreibung der in der Natur beobachteten Hadronen bei niedrigen Energien. Drei dieser Ansätze - effektive Feldtheorie der QCD, QCD im Grenzwert unendlicher Anzahl der Farben und die Gittereichtheorie der QCD - sind Gegenstand dieser Arbeit.

Im Rahmen einer effektiven Feldtheorie, die im Kontext der starken Wechselwirkung auch als Chirale Störungstheorie bezeichnet wird, wird eine störungstheoretische Behandlung der Eigenschaften von Hadronen ermöglicht. Die Entwicklung in der Kopplungskonstante der QCD wird dabei durch eine Entwicklung in kleinen Impulsen der Hadronen und in kleinen Quarkmassen ersetzt.

Die Untersuchung einer physikalischen Theorie in verschiedenen Grenzwerten ist eine gängige Methode in der Physik, um zu einem besseren und tieferen Verständnis der Theorie zu gelangen. Einer der Grenzwerte, in dem die Quantenchromodynamik untersucht werden kann, ist der Grenzwert, bei dem die Anzahl der Farben $N_{c}$ unendlich wird (oder einen großen Wert annimmt). Ergebnisse dieser Formulierung, wenn extrapoliert zum physikalischen Fall mit $N_{c}=3$, beschreiben die Eigenschaften der Hadronen in guter Übereinstimmung mit den empirischen Beobachtungen. Im Lichte dieser Ergebnisse muss der Grenzwert unendlicher Anzahl der Farben als eine viel versprechende Methode für weitere Untersuchungen in der Hadronenphysik angesehen werden.

Die Gittereichtheorie ist eine Formulierung der Eichfeldtheorie, bei der die Regularisierung durch eine Diskretisierung der Raumzeit erreicht wird. Im Rahmen dieser Formulierung für QCD können Eigenschaften der Hadronen durch aufwändige nummerische Simulationen berechnet werden.

Es ist das Ziel dieser Arbeit, das Zusammenspiel von den drei erwähnten Ansätzen am Beispiel der Baryonmassen zu untersuchen. Die für die Berechnung benötigte effektive Wechselwirkung wird diskutiert. Eine Analyse dieser Wechselwirkung im Grenzwert unendlicher Anzahl der Farben in QCD und die daraus resultierenden Relationen und Einschränkungen für die effektiven Kopplungen werden ausgearbeitet. Ein gutes Konvergenzverhalten der chiralen Entwicklung für die Baryonmassen und eine gute Übereinstimmung der chiralen Extrapolation zu höheren Quarkmassen mit den Ergebnissen aktueller Gittereich-Rechnungen wird erreicht. 



\section{Contents}

$\begin{array}{ll}\text { Introduction } & 1\end{array}$

1. QCD at low energy 5

1.1. QCD and its symmetries ..................... 5

1.2. Green functions of QCD and their low-energy expansion . . . . . . . 8

1.3. Effective field theory of QCD . . . . . . . . . . . . . . . 11

1.4. Chiral Lagrangian in the presence of external fields . . . . . . . . . . . . 14

1.4.1. $Q^{1}$ chiral Lagrangian . . . . . . . . . . . . . . . . 16

1.4.2. $Q^{2}$ chiral Lagrangian . . . . . . . . . . . . . . . . . . . . 18

1.4.3. Explicit symmetry breaking . . . . . . . . . . . . 22

2. QCD in the large- $N_{c}$ limit 25

2.1. Introduction . . . . . . . . . . . . . . . . . 25

2.2. Meson and baryon couplings . . . . . . . . . . . . . . . 27

2.3. Baryons in $1 / N_{c}$-expansion . . . . . . . . . . . . . . . . . . 28

2.3.1. Matrix elements of baryon operators . . . . . . . . . . . 29

2.3.2. Operator identities . . . . . . . . . . . . . . 35

2.4. Chiral Lagrangian in the large- $N_{c}$ limit . . . . . . . . . . . . . . . 38

2.4.1. Baryon masses . . . . . . . . . . . . . . . . . . 39

2.4.2. Axial-vector-couplings . . . . . . . . . . . . . . . . . 44

2.4.3. Meson baryon four-point interaction . . . . . . . . . . . 46

3. Quark-mass dependence of the baryon masses 53

3.1. Baryon masses in the chiral loop-expansion . . . . . . . . . . . . . 54

3.2. Chiral extrapolation for the light hadrons . . . . . . . . . . . . . 59

3.2.1. Meson masses . . . . . . . . . . . . . . . . . . . . . 59

3.2.2. Baryon masses . . . . . . . . . . . . . . . . . . . 62

$\begin{array}{ll}\text { Conclusions } & 71\end{array}$

A. One-loop corrections of the baryon masses $\quad 73$

A.1. $Q^{4}$-contributions to the baryon self-energy . . . . . . . . . . . 73

A.2. Scale dependence of the chiral parameters . . . . . . . . . . 75

A.3. Finite terms . . . . . . . . . . . . . . . . . . . . . 78

A.4. Redundancy of the off-shell parameter $Z \ldots \ldots \ldots$. . . . . . 80

B. $S U(3)$ group theory $\quad 85$ 
$\begin{array}{lr}\text { C. Interaction } & \mathbf{8 9}\end{array}$

C.1. SU(3)-invariants . . . . . . . . . . . . . . . . . . . . 89

C.2. Charge conjugation . . . . . . . . . . . . . . . 95

$\begin{array}{ll}\text { D. Baryon states } & 99\end{array}$

D.1. Flavour part . . . . . . . . . . . . . . . . . . . . . . . . . 99

D.2. Spin part . . . . . . . . . . . . . . . . . . 102

E. Matrix elements of spin-flavour operators 105

E.1. Fermionic and bosonic algebras for quarks . . . . . . . . . . . . . . 105

E.2. Normalisation of baryon states . . . . . . . . . . . . . . . 107

E.3. One-body operators . . . . . . . . . . . . . . . . . . . . 108

E.4. Two-body operators I . . . . . . . . . . . . . . . . . . . . . . 112

E.5. Two-body operators, II . . . . . . . . . . . . . . . . . . . . . 114

$\begin{array}{ll}\text { F. Pauli- and spin transition matrices } & 119\end{array}$

G. Operator Identities $\quad 121$

$\begin{array}{ll}\text { H. Spinors } & 129\end{array}$

$\begin{array}{ll}\text { Bibliography } & 141\end{array}$ 


\section{Introduction}

Quantum Chromodynamics (QCD), the theory of strong interaction, is an asymptotically free theory and can be treated perturbatively only at high energies. At low energies the coupling constant of the strong interaction, $g_{s}$, increases rapidly and gives rise to confinement.

The running of the coupling constant invalidates the perturbative expansion at small energies. This problem is overcome in the framework of effective field theory. The concept of effective field theory is a very powerful tool in quantum field theory. In the modern language, the main ideas of this concept can be summarized by the decoupling theorem $[3,73]$ and by Wilson's approach to the program of renormalization in quantum field theory [94]. Given a characteristic energy scale, they show how to separate the physical degrees of freedom below and above this scale and how to incorporate the influence of the physics above the energy scale on the "low-energy" physics. Due to confinement of quarks a perturbative matching of the hadronic and the quark-gluon degrees of freedom at the separation scale is not possible. Therefore, the construction of the effective field theory of QCD, which in this context is referred to as Chiral Perturbation Theory $(\chi \mathrm{PT})$, relies on the principles of quantum field theory and on the symmetries of QCD only. One of the most important ingredient in this approach is the spontaneously and explicitly broken chiral symmetry of quarks, leading to the concept of pseudo-Goldstone bosons in QCD. Those are identified with the lightest pseudo-scalar mesons observed in nature.

At low energies the interaction between the Goldstone bosons is weak in contrast to the strongly interacting quarks and gluons at that energies. This qualitative difference allows a perturbative treatment of the physics of strongly interacting particles. Perturbative expansion in the coupling constant $g_{s}$ is replaced by an expansion in small hadron momenta and quark masses, commonly denoted by the expansion parameter $Q$. This provides a systematic method to describe the hadron interaction at low energy, which, on the other hand, can be related to the matrix elements of quark operators.

To look at the theory in the various limits is a proven tool in physics to extract the information out of it. There are field theories where the inverse power of the number of degrees of freedom $N$ can serve as an expansion parameter. The perturbative expansion in the weak coupling constant is replaced by a topological expansion, where only a certain class of diagrams which survive the large $N$ limit needs to be considered. Some of them become even exactly solvable in the limit where this number $N$ tends to infinity. These ideas were first applied by Stanley in statistical physics [81]. Wilson extended these ideas to quantum field theories [93]. t'Hooft was first to point out, that QCD also possess such an expansion parameter - the number of colours $N_{c}[82,83]$. 
Quantum Chromodynamics greatly simplifies in the limit $N_{c} \rightarrow \infty$ and a systematic expansion of physical observables in powers of $1 / N_{c}$ is possible. One hopes that the theory in this limit still bears the same (or very similar) features as in the physical case $N_{c}=3$. That means that the extension of QCD to the large values of $N_{c}$ can only be predictive if the physical observables have well-defined and physical meaningful behaviour in this limit and if the values of these observables do not change strongly as $N_{c}$ decreases down to the physical value 3 .

Doing the expansion one considers the corrections $\mathcal{O}\left(1 / N_{c}\right)$ to be small (or negligible) for large values of $N_{c}$ and one assumes, that this approximation is also a good approximation for $1 / N_{c}=1 / 3$. The obvious question arising in this context is whether the "coupling" $1 / 3$ should be regarded as "small" or "big". As in many other cases in physics, the quality of an ansatz can be judged only a posteriori. Looking at the results of large- $N_{c}$ calculations and comparing them with the known experimental facts can show, whether the truncation after few leading term in the $1 / N_{c}$-expansion for $N_{c}=3$ is an appropriate approximation to the exact solution or not.

Another framework, making currently huge progress, is the lattice formulation of QCD. In this approach the properties of the gauge theory are determined by large-scale numerical calculations carried out on a discretized Euclidean time-space. There are two types of errors in lattice QCD - statistical and systematical. While the former can be arbitrarily reduced by simply increasing the number of configurations, the later require conceptual efforts. Systematical errors include finite lattice spacing and finite volume effects, fermion formulation on the lattice and chiral extrapolation. The impact of the first two errors on the results can be diminished by making the lattice larger and finer or by "improving" the QCD action, making it less sensitive to the lattice parameters.

In the path-integral formalism, used in the lattice approach to QCD, it is very time consuming to calculate the fermion determinant for the light quarks. Since the first calculation of the hadron spectrum on the lattice [46], for almost two decades the properties of hadrons were calculated in the so-called quenched approximation, where the fermion determinant is set to one. This strongly reduces the run time of numerical calculations. However, it is hard to determine the systematical error of this approximation. An estimation of the error can be provided by the comparison with the lattice calculation carried out with the dynamical (or unquenched) quarks, where the effect of the quarks is taken completely (or partially) into account.

Due to run-time costs, numerical calculations with dynamical quarks have been (and still are in many cases) long time limited to the quark masses artificially set to higher unphysical values. An extrapolation down to the physical point was required in order to compare the results of lattice calculations with the experimental values. Chiral perturbation theory suggests itself as the tool for such kind of extrapolation. Given the quark mass dependence of hadron properties calculated in $\chi \mathrm{PT}$, the extrapolation to the unphysical region is simply obtained by raising the quark masses. Though the validness of $\chi \mathrm{PT}$ in the unphysical region is questionable at the first sight, the range of validity is sometimes claimed to be defined by the region in the quark masses, where $\chi \mathrm{PT}$ is able to describe the lattice data. 
Owing to the constant improvement of the algorithms and the increase of the computational power during the last years, the lower limit of the quark masses available in lattice simulations was strongly moved closer to the physical values. Nowadays even simulations with the dynamical quarks at the physical point become available [2], so that chiral extrapolations - for a long time a compromise due to the lack of computational power - may become obsolete for the lattice community in the near feature. Nevertheless, correct chiral extrapolations, at least in a small region around the physical point, are necessary from the conceptual point of view and allow, furthermore, the determination of the unknown chiral parameters.

It is the aim of this work to confront the chiral extrapolation for the baryon masses with the current lattice calculations using dynamical quarks. Chiral expansion for the baryon masses is calculated within the relativistic formulation of Chiral Perturbation Theory and is supplemented by constraints from the formulation of QCD in the limit of the infinite number of colours. 


\section{Chapter 1.}

\section{QCD at low energy}

Though the formulation of the effective field theory (EFT) approach to the low energy physics of the strong interaction is by now a well established formalism and can be given in almost a canonical form, the description of all details and subtleties is rather lengthy. For a lucid introduction to Chiral Perturbation Theory we reffer to [78], which also containts many references to the original literature.

In the first three sections of this chapter the symmetry properties of QCD are summarised and only the main principles entering the formulation and the construction of the EFT of QCD are outlined. The last section of this chapter contains the effective interaction required for the calculation of the baryon masses up to the forth chiral order.

\subsection{QCD and its symmetries}

The interaction of quarks and gluons is governed by the QCD Lagrangian

$$
\mathcal{L}_{Q C D}=\bar{q}\left(i \gamma^{\mu} D_{\mu}-\mathcal{M}_{q}\right) q-\frac{1}{4} G_{\mu \nu}^{a} G_{a}^{\mu \nu}
$$

with

$$
\begin{aligned}
D_{\mu} & =\partial_{\mu}-g_{s} G_{\mu}^{a} \frac{\lambda^{(a)}}{2} \\
G_{\mu \nu}^{a} & =\partial_{\mu} G_{\nu}^{a}-\partial_{\nu} G_{\mu}^{a}+g_{s} f^{a b c} G_{\mu}^{b} G_{\nu}^{c}
\end{aligned}
$$

Here, $q$ is the quark spin-1/2 field operator, which is in addition a vector in colour and flavour spaces. $G_{\mu}^{a}$ are the eight Yang-Mills gluon fields. The strength of the quark-gluon and gluon-gluon interaction is given by $g_{s}$ and the masses of six known quark flavours up, down, strange, charm, bottom and top - are contained in the mass matrix $\mathcal{M}_{q}$

$$
\mathcal{M}_{q}=\operatorname{diag}\left(m_{u}, m_{d}, m_{s}, m_{c}, m_{b}, m_{t}\right)
$$


usualy, the current quark masses are stated in terms of $\overline{M S}$ renormalised values at $\mu=1$ $\mathrm{GeV}$ (see [78] and the references therein) ${ }^{1}$ :

$$
\left(\begin{array}{c}
m_{u}=0.005 \mathrm{GeV} \\
m_{d}=0.009 \mathrm{GeV} \\
m_{s}=0.175 \mathrm{GeV}
\end{array}\right) \ll 1 \mathrm{GeV} \leq\left(\begin{array}{c}
m_{c}=(1.15-1.35) \mathrm{GeV} \\
m_{b}=(4.0-4.4) \mathrm{GeV} \\
m_{t}=174 \mathrm{GeV}
\end{array}\right) .
$$

In this work only the physics of the three lightest quarks is relevant. In all what follows, talking about quarks only the three lightest flavours are meant, the quark mass matrix is $\mathcal{M}_{q}=\operatorname{diag}\left(m_{u}, m_{d}, m_{s}\right)$ and the quark operator is a three-component vector in the flavour space.

The QCD-Lagrangian respects the discrete $C, P$ and $T$ symmetries separately and is per construction invariant under the $S U(3)$-gauge transformation in the colour space. Furthermore, the strong interaction possesses different (exact, approximate and spontaneously broken) symmetries in the flavour space.

Compared to the masses of the observed hadrons, which QCD is supposed to describe, the running quark masses are very small. Thus, it is reasonable to consider QCD in the limit of vanishing quark masses. In this limit the QCD Lagrange density is, in addition to the already mentioned discrete and gauge symmetries, invariant under the global $U(1)_{V} \otimes S U(3)_{V} \otimes U(1)_{A} \otimes S U(3)_{A}$ flavour transformations of quarks:

$$
q \rightarrow \exp \left(i \theta_{V}+i \theta_{V}^{a} \frac{\lambda^{a}}{2}\right) q, \quad q \rightarrow \exp \left(i \gamma_{5} \theta_{A}+i \gamma_{5} \theta_{A}^{a} \frac{\lambda^{a}}{2}\right) q .
$$

Conserved Noether currents resulting from these transformations are

$$
\begin{aligned}
\mathcal{V}^{\mu} & =\bar{q} \gamma^{\mu} q, & \partial_{\mu} \mathcal{V}^{\mu} & =0, \\
\mathcal{V}^{\mu, a} & =\bar{q} \gamma^{\mu} \frac{\lambda^{a}}{2} q, & \partial_{\mu} \mathcal{V}^{\mu, a} & =0, \\
\mathcal{A}^{\mu, a} & =\bar{q} \gamma^{\mu} \gamma_{5} \frac{\lambda^{a}}{2} q, & \partial_{\mu} \mathcal{A}^{\mu, a} & =0,
\end{aligned}
$$

whereas the $U(1)_{A}$ current $\mathcal{A}^{\mu}=\bar{q} \gamma^{\mu} \gamma_{5} q$ is subject to the Adler-Bell-Jackiw anomaly:

$$
\partial_{\mu} \mathcal{A}^{\mu}=\frac{3 g_{s}^{2}}{32 \pi^{2}} \epsilon_{\mu \nu \rho \sigma} G^{a, \mu \nu} G^{a, \rho \sigma}, \quad \epsilon_{0123}=-\epsilon^{0123}=1 .
$$

The conservation of $\mathcal{A}^{\mu}$ in quantum field theory is broken by instanton effects [84].

\footnotetext{
${ }^{1}$ Due to the confinement of quarks, it is not possible to give the same physical meaning to the mass parameters in (1.1) as in the case of observable particles, say, electron etc. The quark masses are rather regarded as another coupling constants and are treated on the same footing as $g_{s}$. Therefore, the absolute values of the quark masses can only be stated upon having specified the renormalisation scheme and the renormalisation scale. See [34], the contribution of Manohar in [29] and the recent review of Leutwyler [61] for further details and for the current state of the determination of the quark masses.
} 
Conserved currents in (1.6) lead to the associated charge operators

$$
\begin{aligned}
Q_{V}(t) & =\int d^{3} x q^{\dagger}(\vec{x}, t) q(\vec{x}, t), \\
Q_{V}^{a}(t) & =\int d^{3} x q^{\dagger}(\vec{x}, t) \frac{\lambda^{a}}{2} q(\vec{x}, t), \\
Q_{A}^{a}(t) & =\int d^{3} x q^{\dagger}(\vec{x}, t) \gamma_{5} \frac{\lambda^{a}}{2} q(\vec{x}, t),
\end{aligned}
$$

with

$$
\left[Q_{V}^{a}, H_{Q C D}\right]=\left[Q_{A}^{a}, H_{Q C D}\right]=\left[Q_{V}, H_{Q C D}\right]=0 .
$$

The Lie-algebra $S U(3)_{V} \otimes S U(3)_{A}$ in (1.5) is isomorph to the chiral algebra $S U(3)_{R} \otimes$ $S U(3)_{L}$ which corresponds to separate flavour rotations of the right- and left-handed quark components, $q_{R}=\frac{1}{2}\left(1+\gamma_{5}\right) q$ and $q_{L}=\frac{1}{2}\left(1-\gamma_{5}\right) q$, respectively ${ }^{2}$. In the limit of vanishing quark masses, QCD is said to possess a chiral symmetry.

Charge operators are generators of infinitesimal transformations of the symmetry group on the Hilbert space of the theory. Naively one would expect, that the eigenstates of the Hamiltonian should organise themselves in multiplets which are degenerate in quantum numbers associated with the charges. As it can be shown (see e.g. [78]), this statement is only true if the charge operators leave the ground state of the theory invariant ${ }^{3}$.

Concerning the charge operators $Q_{V}^{a}$ and $Q_{A}^{a}$ in (1.8), no chiral doublets - particles equal in mass, spin, baryon and flavour quantum numbers and opposite in parity - are observed in the hadron spectrum. On the other hand, the observed low-energy baryons with positive parity can be approximately organised in different $S U(3)$ multiplets. This suggests, that only the $S U(3)_{V}$ subgroup of $S U(3)_{V} \otimes S U(3)_{A}$ is a symmetry group of the states.

A symmetry of the Hamiltonian, that is not realised in the Hilbert space, is said to be spontaneously broken. From the observation in the hadron spectrum one concludes, that in the limit $\mathcal{M}_{q}=0$ the full chiral symmetry group of QCD is spontaneously broken to its vector subgroup:

$$
S U(3)_{R} \otimes S U(3)_{L}=S U(3)_{V} \otimes S U(3)_{A} \rightarrow S U(3)_{V} .
$$

This fact can equivalently be stated as

$$
Q_{A}^{a}|0\rangle \neq 0 \text {. }
$$

${ }^{2}$ The chiral operators $P_{R}=\frac{1}{2}\left(1+\gamma_{5}\right)$ and $P_{L}=\frac{1}{2}\left(1-\gamma_{5}\right)$ are projectors:

$$
P_{R}+P_{L}=1, \quad P_{R}^{2}=P_{R}, \quad P_{L}^{2}=P_{L}, \quad P_{R} P_{L}=P_{L} P_{R}=0 .
$$

They project onto the field components of different chirality: $\gamma_{5} q_{R, L}= \pm q_{R, L}$. In the zero-mass limit of the free quarks, the chiral operators also project onto the eigenstates of the helicity operator $h=$ $\vec{\sigma} \cdot \vec{p} / \sqrt{\vec{p}^{2}}: \quad h q_{R, L}= \pm q_{R, L}$. I.e., chirality is equal to helicity in this limit. The terminology right- and left-handed used here is based on this fact.

${ }^{3}$ The opposite case is always true - the invariance of the vacuum under transformations of some symmetry group implies always the invariance of the Hamiltonian under the same symmetry group, but not vice versa [17]. 
According to Goldstone's theorem, for each generator in the broken subgroup there is a massless boson carrying the quantum numbers of the broken generator. For the spontaneously broken $S U(3)_{A}$ with 8 generators one expects to find eight massless mesons in the hadron spectrum. These Goldstone bosons are identified with the eight lightest pseudoscalar mesons $\pi^{+}, \pi^{-}, \pi^{0}, K^{+}, K^{-}, K^{0}, \bar{K}^{0}, \eta$. The key assumption which lead to the idea of Goldstone bosons in QCD was the chiral limit of the theory, which is only approximately realised in nature. Therefore, the pseudoscalar mesons mentioned above, acquire small (as compared to other observed pseudoscalar mesons) but non-vanishing masses and are often called pseudo-Goldstone bosons.

The $U(1)_{V}$ symmetry of QCD is identified with the baryon number conservation. The eigenstates of $Q_{V}$ are mesons and baryons. The corresponding $U(1)_{V}$ "charge", the baryon number $B$, is $B=0$ and $B=1$ for mesons and baryons, respectively.

In the presence of the quark mass term in the QCD Lagrangian the divergences of the currents in (1.6) are modified as follows:

$$
\begin{aligned}
\partial_{\mu} V^{\mu} & =0 \\
\partial_{\mu} \mathcal{A}^{\mu} & =2 i \bar{q} \mathcal{M}_{q} \gamma_{5} q+\frac{3 g_{s}^{2}}{32 \pi^{2}} \epsilon_{\mu \nu \rho \sigma} G^{a, \mu \nu} G^{a, \rho \sigma} \\
\partial_{\mu} V^{\mu, a} & =i \bar{q}\left[\mathcal{M}_{q}, \frac{\lambda^{a}}{2}\right] q \\
\partial_{\mu} A^{\mu, a} & =i \bar{q}\left\{\mathcal{M}_{q}, \frac{\lambda^{a}}{2}\right\} \gamma_{5} q .
\end{aligned}
$$

The $U(1)_{V}$ symmetry of QCD is not affected by the quark masses. Assuming the masses of the three lightest quarks to have the same value, $m_{u}=m_{d}=m_{s}$, the quark mass matrix $\mathcal{M}_{q}$ commutes with all generators of $S U(3)$. The conservation of the octet vector current in this limit corresponds to the approximate $S U(3)$ flavour symmetry of baryons which was suggested by Gell-Mann on the ground of phenomenological observations and which lead to the idea of quarks as the building elements of hadrons [42].

Furthermore, from the last line of (1.13) it follows, that the divergence of the axial-vector current is proportional to a pseudoscalar quantity. This is the modern explanation of the PCAC relation - partially conservation of the axial-vector current.

\subsection{Green functions of QCD and their low-energy expansion}

The objects of main interest in QCD are the vacuum matrix elements of time ordered quark operators, the quark Green functions. On the level of Green functions, the symmetry properties of the QCD Lagrangian are expressed by a set of Ward identities. The expansion of Green functions in powers of the external momenta and of the quark masses is parametrised in terms of some coefficients. These coefficients are functions of the strong coupling constant $\alpha_{s}$ and the quark masses only (the only free parameters of QCD) and 
are interrelated to each other by the Ward identities. The expansion coefficients may be identified with the coupling constants of an unique effective Lagrangian. Starting with such an effective Lagrangian, governed by the general principles of quantum field theory and by the symmetry constraints of the underlying theory, the low-energy representation of the Green function can be calculated more easily. The connection of QCD Green functions and their low-energy expansion to the effective Lagrangian was systematically worked out by Gasser and Leutwyler in a series of papers [36, 35, 37, 38].

The technique of external fields provides a systematic method to derive Green functions and to study relations between them. Quark currents in question - vector, axial vector, scalar and pseudoscalar - are coupled to external c-number sources as in

$$
\begin{aligned}
\mathcal{L}_{Q C D}(G, \bar{q}, q ; v, a, s, p) & \equiv \mathcal{L}_{Q C D}^{0}+\mathcal{L}_{Q C D}^{\mathrm{ext}} \\
& \equiv \mathcal{L}_{Q C D}^{0}+\bar{q}\left(\gamma_{\mu}\left(v^{\mu}+\gamma_{5} a^{\mu}\right)-\left(s-i p \gamma_{5}\right)\right) q,
\end{aligned}
$$

with $\mathcal{L}_{Q C D}^{0}$ given by the Lagrangian in (1.1) in the chiral limit. The vector field $v_{\mu}$, the axial-vector field $a_{\mu}$ and the scalar and pseudo-scalar fields $s$ and $p$, respectively, are hermitian matrix-valued fields in flavour space ${ }^{4}$ :

$$
v_{\mu}=\sum_{a=1}^{8} \frac{\lambda^{a}}{2} v_{\mu}^{a}, \quad a_{\mu}=\sum_{a=1}^{8} \frac{\lambda^{a}}{2} a_{\mu}^{a}, \quad s=s^{0} \mathbb{1}+\sum_{a=1}^{8} \lambda^{a} s^{a}, \quad p=p^{0} \mathbb{1}+\sum_{a=1}^{8} \lambda^{a} p^{a} .
$$

The original $\mathcal{L}_{Q C D}$ is restored by setting $v_{\mu}=a_{\mu}=p=0$ and $s=\mathcal{M}_{q}$ in (1.14). The generating functional (vacuum-to-vacuum transition amplitude) in the presence of external fields is given by $^{5}$

$$
\mathcal{Z}[v, a, s, p]=e^{i W[v, a, s, p]}=\left\langle 0_{\text {out }} \mid 0_{\text {in }}\right\rangle_{v, a, s, p},
$$

and is calculated on the basis of (1.14). Green functions are obtained by taking functional derivatives of the generating functional with respect to external fields:

$$
\begin{aligned}
\langle 0|\bar{q}(x) q(x)| 0\rangle & =\left.i \frac{\delta}{\delta s^{0}(x)} \mathcal{Z}[v, a, s, p]\right|_{v=a=p=0, s=\mathcal{M}_{q}}, \\
\left\langle 0\left|\bar{q}(x) \gamma^{\mu} \gamma_{5} \frac{\lambda^{a}}{2} q(x)\right| 0\right\rangle & =-\left.i \frac{\delta}{\delta a_{\mu}^{a}(x)} \mathcal{Z}[v, a, s, p]\right|_{v=a=p=0, s=\mathcal{M}_{q}}, \\
\left\langle 0\left|\mathcal{T} \bar{q}(x) \gamma^{\mu} \gamma_{5} \frac{\lambda^{a}}{2} q(x) \bar{q}(y) \gamma^{\nu} \gamma_{5} \frac{\lambda^{b}}{2} q(y)\right| 0\right\rangle & =-\left.\frac{\delta}{\delta a_{\mu}^{a}(x)} \frac{\delta}{\delta a_{\nu}^{b}(y)} \mathcal{Z}[v, a, s, p]\right|_{v=a=p=0, s=\mathcal{M}_{q}}, \\
\text { etc. } &
\end{aligned}
$$

In the presence of external fields, the QCD Lagrange density is invariant under the local $S U(3)_{R} \otimes S U(3)_{L}$ transformations of right- and left-handed quarks,

$$
q_{R}(x) \mapsto V_{R}(x) q_{R}(x), \quad q_{L}(x) \mapsto V_{L}(x) q_{L}(x),
$$

\footnotetext{
${ }^{4}$ As long as we want to study $S U(3)$ vector and axial-vector currents, the flavour singlet components in $v_{\mu}$ and $a_{\mu}$ can be disregarded.

${ }^{5}$ In opposite to the works [36, 35, 37], the generating functional of connected Green functions is denoted in this work by $W$, whereas $\mathcal{Z}$ is reserved for the generating functional of all Green functions as in many textbooks (e.g. [47, 75, 91, 77, 18]).
} 
provided the external fields transform as

$$
\begin{aligned}
r_{\mu}(x) & \mapsto V_{R}(x) r_{\mu}(x) V_{R}^{\dagger}(x)+i V_{R}(x) \partial_{\mu} V_{R}^{\dagger}(x), \\
l_{\mu}(x) & \mapsto V_{L}(x) r_{\mu}(x) V_{L}^{\dagger}(x)+i V_{L}(x) \partial_{\mu} V_{L}^{\dagger}(x), \\
s(x)+i p(x) & \mapsto V_{R}(x)(s(x)+i p(x)) V_{L}^{\dagger}(x),
\end{aligned}
$$

with $r_{\mu}=v_{\mu}+a_{\mu}$ und $l_{\mu}=v_{\mu}-a_{\mu}$.

The information, carried by the chiral quark Ward identities, is embodied in the transformation properties of the generating functional under the gauge transformations in (1.18) and (1.19). In the absence of anomalies, the conservation of quark currents is expressed by the gauge invariance of $\mathcal{Z}$. Due to anomalies the generating functional is not invariant under the full chiral transformations stated above. The non-vanishing change in $\mathcal{Z}$ as the result of chiral gauge transformation can be worked out explicitly explicitly [5, 92, 37]. At the formal level, the generating functional can be splitted in two parts:

$$
\mathcal{Z}[v, a, s, p]=\mathcal{Z}[v, a, s, p]_{\mathrm{inv}}+\mathcal{Z}[v, a, s, p]_{\mathrm{anom}} .
$$

All Green functions obtained form $\mathcal{Z}$ automatically satisfy the corresponding (anomalous and non-anomalous) Ward identities.

Of special interest are quark operators taken between baryon states instead of vacuum. Following [39], we denote the baryon-baryon transition amplitude in the presence of external fields by

$$
\mathcal{F}\left(\vec{p}^{\prime}, \vec{p} ; v, a, s, p\right)=\left\langle\bar{p}_{\text {out }} \mid \vec{p}_{\text {in }}\right\rangle_{v, a, s, p}^{\text {connected }}, \quad \vec{p}^{\prime} \neq \vec{p},
$$

where $\left|\vec{p}_{\text {in }}\right\rangle\left(\left|\vec{p}_{\text {out }}^{\prime}\right\rangle\right)$ is the incoming (outgoing) one-baryon state with the momentum $\vec{p}\left(\vec{p}^{\prime}\right)$. Similar to (1.16), this amplitude serves as a generating functional for the baryonic matrix elements of various quark operators. Analogous to (1.17), the low-energy expansion of them is obtained by taking functional derivatives of $\mathcal{F}$ calculated with the help of an effective Lagrangian.

In this work we are solely concerned with the baryon states which are members of the flavour $S U(3)$ octet and decuplet multiplets. These states are completely specified by the four-momentum $p$, the spin-polarization index $\chi$ and by the flavour indices $a$ and $i, j, k$, respectively:

$$
|p, \chi, a\rangle, \quad \text { and } \quad|p, \chi, i j k\rangle \text {. }
$$

Here, the spin-polarization index takes the values $\chi=1,2$ for the spin- $1 / 2$ baryons of the octet and $\chi=1, \cdots, 4$ for the spin-3/2 baryons of the decuplet and $a=1, \cdots, 8$ and $i, j, k=1,2,3$, respectively.

The low-energy expansion of the Green functions and of the baryonic matrix elements of quark currents corresponds to an expansion in small quark masses and small external momenta. This amounts to an expansion of the corresponding generating functionals, 
$\mathcal{Z}$ or $\mathcal{F}$, in powers of the external fields and derivatives of them. An effective way to determine such an expansion for the matrix elements of operators with given symmetry properties provide effective Lagrangians $[23,20,89,19,16,90,37]$. In the next section the effective Lagrangian for QCD at low energy will be discussed. In doing so, the discussion will be limited to the part of the effective Lagrangian which amounts for the invariant part of the generating functional in (1.20). The anomalous part can be given in an explicit form [92], but is of no relevance in this work.

\subsection{Effective field theory of QCD}

$\mathcal{L}_{Q C D}$ with the symmetry group $S U(3)_{R} \otimes S U(3)_{L} \otimes U(1)_{V}$, which is spontaneously broken to its subgroup $S U(3)_{V} \times U(1)_{V}$, and the discrete symmetries of QCD serve as the starting point of the construction of the effective field theory. Rewriting the theory in terms of the hadronic degrees of freedom makes the introduction of a non-linear realisation of the symmetry group for the Goldstone-bosons necessary. The case of the spontaneous breakdown of the chiral $S U(2)_{R} \otimes S U(2)_{L}$ group to $S U(2)_{V}$ was originally discussed by Weinberg in [89]. The generalisation to arbitrary symmetry groups which are spontaneously broken to some subgroup and the transformation properties of Goldstone boson and matter fields ${ }^{6}$ was done by Callan, Colleman, Wess and Zumino (CCWZ) $[19,16]$.

The pseudo-scalar quantum fields for eight pseudo-Goldstone-bosons, which result from the approximate spontaneous breakdown of the chiral symmetry $S U(3)_{R} \otimes S U(3)_{L}$, will be denoted by $\phi_{a}(a=1, \ldots 8)$. According to the CCWZ-prescription, they can be parametrised non-linearly ${ }^{7}$ by a $S U(3)$-matrix $U$

$$
U=e^{i \Phi / f}, \quad U^{\dagger} U=1, \operatorname{det}(U)=1,
$$

with

$$
\Phi=\sum_{a=1}^{8} \phi_{a} \lambda^{(a)}=\left(\begin{array}{ccc}
\pi^{0}+\frac{1}{\sqrt{3}} \eta & \sqrt{2} \pi^{+} & \sqrt{2} K^{+} \\
\sqrt{2} \pi^{-} & -\pi^{0}+\frac{1}{\sqrt{3}} \eta & \sqrt{2} K^{0} \\
\sqrt{2} K^{-} & \sqrt{2} \bar{K}^{0} & -\frac{2}{\sqrt{3}} \eta
\end{array}\right) .
$$

Matrix elements of the hermitian matrix $\Phi$ are identified with the physical fields by comparing the isospin- and strangeness quantum numbers of the $S U(3)$ octet $\Phi$ with those of the observed pseudoscalar mesons (see e.g. [33]). The parameter $f$ with the mass dimension one, introduced in (1.23) to make the exponent dimensionless, is related with the weak decay constant of the Goldstone-bosons.

\footnotetext{
${ }^{6}$ In the CCWZ-formalism, the matter fields are fields which are not the Goldstone bosons.

${ }^{7}$ There are infinitely many possibilities to introduce fields, associated with the Goldstone bosons, non-linearly. The exponential representation in (1.23) is the most convenient one. Though, the different parametrisation lead to different Green functions, the S-matrix elements are invariant under parametrisation $[45,55]$.
} 
In contrast to the fields $\phi_{a}$, the matrix $U$ transforms linearly under the chiral group $S U(3)_{R} \otimes S U(3)_{L}:$

$$
U \rightarrow U^{\prime}=V_{R} U V_{L}^{\dagger}, \quad V_{R} \in S U(3)_{R}, V_{L} \in S U(3)_{L} .
$$

In this work, the relevant matter fields are the $\frac{1}{2}^{+}$and $\frac{3}{2}^{+}$baryons building a $S U(3)$ octet and decuplet, respectively:

$$
B=\frac{1}{\sqrt{2}} \sum_{a=1}^{8} b_{a} \lambda^{(a)}, \quad \Delta_{i j k}^{\mu}, \quad i, j, k=1,2,3 .
$$

The components of the flavour tensors $B$ and $\Delta$ are related to the physical particles as in $^{8}$

$$
B=\left(\begin{array}{ccc}
\frac{1}{\sqrt{2}} \Sigma^{0}+\frac{1}{\sqrt{6}} \Lambda & \Sigma^{+} & p \\
\Sigma^{-} & -\frac{1}{\sqrt{2}} \Sigma^{0}+\frac{1}{\sqrt{6}} \Lambda & n \\
-\Xi^{-} & \Xi^{0} & -\frac{2}{\sqrt{6}} \Lambda
\end{array}\right)
$$

and

$$
\begin{aligned}
& \Delta_{111}=\Delta^{++}, \quad \Delta_{112}=\Delta^{+} / \sqrt{3}, \quad \Delta_{122}=\Delta^{0} / \sqrt{3}, \quad \Delta_{222}=\Delta^{-}, \\
& \Delta_{113}=\Sigma^{+} / \sqrt{3}, \quad \Delta_{123}=\Sigma^{0} / \sqrt{6}, \quad \Delta_{223}=\Sigma^{-} / \sqrt{3}, \\
& \Delta_{133}=\Xi^{0} / \sqrt{3}, \quad \Delta_{233}=\Xi^{-} / \sqrt{3}, \\
& \Delta_{333}=\Omega^{-}
\end{aligned}
$$

Transformation of the baryon fields under the chiral symmetry group is not fixed. Baryon field operators with different transformation properties can be related to each other by field redefinitions ${ }^{9}$. A convenient way to set the transformation of baryons is

$$
B \rightarrow B^{\prime}=R B R^{\dagger}, \quad \Delta_{\mu}^{i j k} \rightarrow \Delta_{\mu}^{i j k \prime}=R_{l}^{i} R_{m}^{j} R_{n}^{k} \Delta_{\mu}^{l m n} .
$$

The transformation matrix $R$ is defined implicitly via

$$
u^{\prime}=V_{R} u R^{\dagger}=R u V_{L}^{\dagger}, \quad \text { with } \quad u^{2}=U, u=\exp \left(\frac{i}{2 f} \Phi\right) .
$$

The matrix $R$ depends on $V_{R}, V_{L}, U$ and on $x$ through the coordinate dependence of the meson fields in $U$. Though not obvious at the first sight, the transformation of baryons in (1.29) leads to a derivative coupling of the Goldstone bosons to the baryons. The fact, that the hadrons are non-interacting in the limit of vanishing momenta - one of the most

\footnotetext{
${ }^{8}$ The flavour matrix $B$ possess a mixed flavour symmetry, whereas the tensor $\Delta$ is completely symmetric under interchange of the flavour indices (s. a. Appendix D).

${ }^{9}$ The reparametrization invariance of the S-matrix permit the introduction of baryon fields, which transform in an appropriate and in a more convenient way. The physical predictions are independent of the specific form of the baryon field operator. The freedom to introduce the baryon fields with specific chiral transformation properties is analogous to the freedom to define a non-linear representation of the mesons as in (1.23).
} 
important consequences of the chiral limit - is more explicit in this form.

The invariance of the generating functional of the theory under the local chiral transformations makes the introduction of covariant derivatives necessary, which transform under local $S U(3)_{R} \otimes S U(3)_{L}$ group in the same way as the objects they act on. It holds:

$$
\begin{aligned}
\nabla_{\mu} U & =\partial_{\mu} U-i r_{\mu} U+i U l_{\mu} \\
\nabla_{\mu} U^{\dagger} & =\partial_{\mu} U^{\dagger}+i U^{\dagger} r_{\mu}-i l_{\mu} U^{\dagger} \\
D_{\mu} B & =\partial_{\mu} B+\Gamma_{\mu} B+B \Gamma_{\mu}^{\dagger}=\partial_{\mu} B+\left[\Gamma_{\mu}, B\right] \\
D_{\mu} \Delta_{\nu}^{i j k} & =\partial_{\mu} \Delta_{\nu}^{i j k}+\left(\Gamma_{\mu}\right)_{l}^{i} \Delta_{\nu}^{l j k}+\left(\Gamma_{\mu}\right)_{l}^{j} \Delta_{\nu}^{i l k}+\left(\Gamma_{\mu}\right)_{l}^{k} \Delta_{\nu}^{i j l} .
\end{aligned}
$$

The connection $\Gamma_{\mu}$, which enters the definition of the covariant derivative for the baryons, is given by

$$
\begin{aligned}
\Gamma_{\mu} & =\frac{1}{2}\left(u^{\dagger}\left(\partial_{\mu}-i r_{\mu}\right) u+u\left(\partial_{\mu}-i l_{\mu}\right) u^{\dagger}\right) \\
& =\frac{1}{2}\left[u^{\dagger}, \partial_{\mu} u\right]-\frac{i}{2}\left(u^{\dagger} r_{\mu} u+u l_{\mu} u^{\dagger}\right),
\end{aligned}
$$

with

$$
\Gamma_{\mu}^{\dagger}=-\Gamma_{\mu}, \quad \text { and } \quad \Gamma_{\mu} \stackrel{S U(3)_{R} \otimes S U(3)_{L}}{\longrightarrow} R \Gamma_{\mu} R^{\dagger}+R \partial_{\mu} R^{\dagger} .
$$

In the process of promoting a global symmetry to a local one, the interaction of the gauge fields is taken into account via the corresponding field stress tensors ${ }^{10}$ :

$$
f_{\mu \nu}^{R}=\partial_{\mu} r_{\nu}-\partial_{\nu} r_{\mu}-i\left[r_{\mu}, r_{\nu}\right], \quad f_{\mu \nu}^{L}=\partial_{\mu} l_{\nu}-\partial_{\nu} l_{\mu}-i\left[l_{\mu}, l_{\nu}\right],
$$

with

$$
f_{\mu \nu}^{R} \stackrel{S U(3)_{R} \otimes S U(3)_{L}}{\longrightarrow} V_{R} f_{\mu \nu}^{R} V_{R}^{\dagger}, \quad f_{\mu \nu}^{L} \stackrel{S U(3)_{R} \otimes S U(3)_{L}}{\longrightarrow} V_{L} f_{\mu \nu}^{R} V_{L}^{\dagger} .
$$

For the scalar and pseudoscalar fields one introduces ${ }^{11}$ :

$$
\chi_{0}=2 B_{0}(s+i p), \quad \text { with } \quad \chi_{0} \stackrel{S U(3)_{R} \otimes S U(3)_{L}}{\longrightarrow} V_{R} \chi_{0} V_{L}^{\dagger} .
$$

In the course of the construction of the effective interaction, it is more convenient to deal with objects, which transform all in the same way, e.g. as the baryons in (1.29). In order to achieve this, one introduces the following objects for the mesons and for the external fields:

$$
\begin{aligned}
U_{\mu} & =\frac{1}{2} u^{\dagger}\left(\nabla_{\mu} U\right) u^{\dagger}=-\frac{1}{2} u\left(\nabla_{\mu} U\right)^{\dagger} u, \\
f_{\mu \nu}^{ \pm} & =u^{\dagger} f_{\mu \nu}^{R} u \pm u f_{\mu \nu}^{L} u^{\dagger}, \\
\chi_{ \pm} & =\frac{1}{2}\left(u \chi_{0}^{\dagger} u \pm u^{\dagger} \chi_{0} u^{\dagger}\right) .
\end{aligned}
$$

\footnotetext{
${ }^{10}$ External fields, introduced here as a technical tool to access matrix elements of various quark operators, can be identified with the electroweak gauge fields of the Standard Model (see e.g. [78]). This allows to study electroweak properties of quarks and hadrons.

${ }^{11}$ The parameter $B_{0}$ used in the definition of $\chi_{0}$ is related to the order parameter of the chiral symmetry breaking in QCD: $3 f^{2} B_{0}=-\langle 0|\bar{q} q| 0\rangle$. The quark condensate can be determined e.g. by using lattice or QCD sum rule methods.
} 


\begin{tabular}{|c|c|c|c|c|}
\hline & $S U(3)_{R} \otimes S U(3)_{L}$ & $C$ & $\Lambda$ & h.c. \\
\hline$U_{\mu}$ & $R u_{\mu} R^{\dagger}$ & $u_{\mu}^{T}$ & $(\operatorname{det} \Lambda) \Lambda_{\mu}^{\nu} u_{\nu}$ & $-u_{\mu}$ \\
$\chi_{+}$ & $R \chi_{+} R^{\dagger}$ & $\chi_{+}^{T}$ & $\chi_{+}$ & $\chi_{+}$ \\
$\chi_{-}$ & $R \chi_{-} R^{\dagger}$ & $\chi_{-}^{T}$ & $(\operatorname{det} \Lambda) \chi_{-}$ & $-\chi_{-}$ \\
$f_{\mu \nu}^{+}$ & $R f_{\mu \nu}^{+} R^{\dagger}$ & $-f_{\mu \nu}^{+T}$ & $\Lambda_{\mu}^{\alpha} \Lambda_{\nu}^{\beta} f_{\alpha \beta}^{+}$ & $f_{\mu \nu}^{+}$ \\
$f_{\mu \nu}^{-}$ & $R f_{\mu \nu}^{-} R^{\dagger}$ & $f_{\mu \nu}^{-T}$ & $(\operatorname{det} \Lambda) \Lambda_{\mu}^{\alpha} \Lambda_{\nu}^{\beta} f_{\alpha \beta}^{-}$ & $f_{\mu \nu}^{-}$ \\
\hline
\end{tabular}

Table 1.1.: Transformation properties of $U_{\mu}, \chi_{ \pm}, f_{\mu \nu}^{ \pm}$.

The transformation properties of these building blocks under chiral transformation, charge conjugation, general Lorentz transformation $\Lambda$ as well as their hermitian conjugates are summarised in Table (1.1).

\subsection{Chiral Lagrangian in the presence of external fields}

The most general chiral Lagrangian is obtained with the help of the building blocks introduced in the previous section, with the derivatives on the meson and baryon fields replaced with the pertinent covariant expressions. Products built out of them transform non-linearly under $S U(3)_{R} \otimes S U(3)_{L}$ as $R \ldots R^{\dagger}$. To guarantee the invariance of such products, traces in flavour space have to be taken. The so obtained interaction terms are $S U(3)$-scalars. Furthermore, it has to be ensured, that they are also scalars in Lorentz- and Dirac-spaces and are invariant under the discrete $C, P, T$ symmetry transformations.

The symmetry constraints for the effective Lagrangian still allow an infinite number of interaction terms. To achieve a systematic low-energy expansion of the generating functionals discussed in Section 1.2, a power counting scheme needs to be established. The interaction terms in the chiral Lagrangian are grouped according to the low-energy expansion parameter $Q$, which stands either for small external momenta or for the quark masses. The building blocks are booked as

$$
U, u, B=\mathcal{O}\left(Q^{0}\right), \quad v_{\mu}, a_{\mu}=\mathcal{O}\left(Q^{0}\right), \quad U_{\mu}=\mathcal{O}\left(Q^{1}\right), \quad f_{\mu \nu}^{ \pm}, \chi_{ \pm}=\mathcal{O}\left(Q^{2}\right) .
$$

While the spatial three-momentum of the baryons in the low-energy region can be regarded as small, the four-momentum cannot be treated as a small quantity since the baryon mass doesn't vanish in the chiral limit. The derivatives and the Dirac-operator are counted as

$$
D_{\mu} B=\mathcal{O}\left(Q^{0}\right), \quad\left(i \gamma^{\mu} D_{\mu}-\stackrel{\circ}{M}\right) B=\mathcal{O}\left(Q^{1}\right) .
$$

Furthermore, the study of the spatial momentum dependence of the elements of the Clifford algebra leads to the following counting

$$
\mathbb{I}, \gamma_{\mu}, \gamma_{5} \gamma_{\mu}, \sigma_{\mu \nu}=\mathcal{O}\left(Q^{0}\right), \quad \gamma_{5}=\mathcal{O}\left(Q^{1}\right) .
$$


The minimal chiral order of a given diagram is $Q^{\nu}$ with

$$
\left.\nu=\sum_{i} V_{i}\left(d_{i}+2 m_{i}\right)-2 I_{M}-I_{B}+4 L\right) .
$$

Here, the summation is done over all types of vertices. $V_{i}$ is the number of vertices of type $i, d_{i}$ and the $m_{i}$ are the number of derivatives on the meson fields and the number of the matrices $f_{\mu \nu}$ and $\chi_{ \pm}$in that vertex, respectively. Furthermore, $I_{M}$ and $I_{B}$ stand for the number of mesonic and baryonic inner lines and $L$ counts the number of loops. Given a chiral order $\nu$, the relevant interaction terms and the diagramms are determined with the help of (1.41).

At every order of the low-energy expansion a new set of unknown constants appears. These constants are functions of QCD parameters $-g_{s}$ and the quark masses. For the lack of ability of a direct calculation of these constants, they have to be fitted to physical observables.

General rules for the construction of the chiral $S U(3)$ Lagrangian, transformation properties of the interaction terms containing an arbitrary number of meson fields and the interaction up to the chiral order $Q^{3}$ were discussed in detail by Krause in [57]. This work was recently reviewed, and completed in [71, 31, 72]. The analysis in these works was carried out for $S U(3)$ flavour octet baryons only.

The extension of the general results obtained in the cited works to the spin-3/2 decuplet baryons is, in principle, straightforward. The generalisation to the arbitrary number of mesons is complicated by the fact that all possible flavour contractions are not so obvious at the first sight. In the case of decuplet baryons, the determination of the $S U(3)$-singlets has to be done for every case of interest separately. Appendix $\mathrm{C}$ contains an extensive discussion of this topic.

In this section we state the chiral interaction required for the calculation of the baryon masses up to the fourth chiral order. To obtain the relevant terms, the interaction, stated in terms of the building blocks, is further expanded in mesonic and external fields and is truncated at the relevant order with the help of

$$
\begin{aligned}
U_{\mu} & =\frac{i}{2 f} \partial_{\mu} \Phi-\frac{1}{2 f}\left[\Phi, v_{\mu}\right]-i a_{\mu}+\mathcal{O}\left(\Phi^{2}\right), \\
\Gamma_{\mu} & =\frac{1}{8 f^{2}}\left[\Phi, \partial_{\mu} \Phi\right]-i v_{\mu}+\frac{1}{2 f}\left[a_{\mu}, \Phi\right]+\frac{i}{8 f^{2}}\left[\Phi,\left[\Phi, v_{\mu}\right]\right]+\mathcal{O}\left(\Phi^{3}\right), \\
f_{\mu \nu}^{ \pm} & =f_{\mu \nu}^{R} \pm f_{\mu \nu}^{L}+\frac{i}{2 f}\left[f_{\mu \nu}^{R} \mp f_{\mu \nu}^{L}, \Phi\right]-\frac{1}{8 f^{2}}\left[\Phi,\left[\Phi, f_{\mu \nu}^{R} \pm f_{\mu \nu}^{L}\right]\right]+\mathcal{O}\left(\Phi^{3}\right), \\
\chi_{+} & =\chi_{0}-\frac{1}{8 f^{2}}\left\{\Phi,\left\{\Phi, \chi_{0}\right\}\right\}+\mathcal{O}\left(\Phi^{3}\right), \\
\chi_{-} & =\frac{i}{2 f}\left\{\chi_{0}, \Phi\right\}+\mathcal{O}\left(\Phi^{3}\right),
\end{aligned}
$$


and

$$
\begin{aligned}
f_{\mu \nu}^{R}+f_{\mu \nu}^{L} & =2 f_{\mu \nu}^{V}+2 i\left[a_{\mu}, a_{\nu}\right] \\
f_{\mu \nu}^{R}-f_{\mu \nu}^{L} & =2\left(\partial_{\mu} a_{\nu}-\partial_{\nu} a_{\mu}\right)-2 i\left(\left[v_{\mu}, a_{\nu}\right]+\left[a_{\mu}, v_{\nu}\right]\right) .
\end{aligned}
$$

To cope with flavour indices in the products of $S U(3)$ tensors containing decuplet, we use following compact dot-notation introduced in [64]:

$$
(\bar{\Delta} \cdot \Delta)_{k}^{m} \equiv \bar{\Delta}_{i j k} \Delta^{i j m}, \quad(\bar{\Delta} \cdot \Phi)_{k}^{m} \equiv \bar{\Delta}_{i j k} \Phi_{l}^{i} \epsilon^{j l m}, \quad(\Phi \cdot \Delta)_{k}^{m} \equiv \Delta^{i j m} \Phi_{i}^{l} \epsilon_{j l k} .
$$

\subsection{1. $Q^{1}$ chiral Lagrangian}

Leading order chiral Lagrangian is of the order one and is given by

$$
\begin{aligned}
\mathcal{L}^{(1)} & =\operatorname{tr}\left(\bar{B}\left(i \not D-\stackrel{\circ}{M}_{[8]}\right) B\right) \\
& -\operatorname{tr}\left(\bar{\Delta}_{\mu} \cdot\left(\left(i \not D-\stackrel{\circ}{M}_{[10]}\right) g^{\mu \nu}-i\left(\gamma^{\mu} D^{\nu}+\gamma^{\nu} D^{\mu}\right)+\gamma^{\mu}\left(i \not D+\stackrel{\circ}{M}_{[10]}\right) \gamma^{\nu}\right) \Delta_{\nu}\right) \\
& +F \operatorname{tr}\left(\bar{B} \gamma^{\mu} \gamma_{5}\left[i U_{\mu}, B\right]\right)+D \operatorname{tr}\left(\bar{B} \gamma^{\mu} \gamma_{5}\left\{i U_{\mu}, B\right\}\right) \\
& +C\left(\operatorname{tr}\left(\left(\bar{\Delta}_{\mu} \cdot i U^{\mu}\right) B\right)+\text { h.c. }\right)+H \operatorname{tr}\left(\left(\bar{\Delta}^{\mu} \cdot \gamma_{\nu} \gamma_{5} \Delta_{\mu}\right) i U^{\nu}\right) .
\end{aligned}
$$

The first two lines are the covariant generalisation of the spin- $1 / 2$ and spin- $3 / 2$ kinetic and mass terms for objects carrying $S U(3)$ indices $^{12}$. The mass parameters $\stackrel{\circ}{M}_{[8]}$ and $\stackrel{\circ}{M}[10]$ are the masses of octet and decuplet baryons in the chiral limit, respectively.

The interaction stated in the last two lines of (1.45) describes axial properties of baryons and the coupling of the Goldstone bosons to them. Further meson-baryon interaction terms are obtained by using the expansion of the building blocks in (1.42). Putting the results for $\Gamma_{\mu}$ into the kinetic terms, one obtains for the octet baryons

$$
\begin{aligned}
\operatorname{tr}\left(\bar{B} i \gamma^{\mu} D_{\mu} B\right) & =\operatorname{tr}\left(\bar{B} i \gamma^{\mu} \partial_{\mu} B\right)+\frac{i}{8 f^{2}} \operatorname{tr}\left(\bar{B} \gamma^{\mu}\left[\left[\Phi, \partial_{\mu} \Phi\right], B\right]\right) \\
& +\operatorname{tr}\left(\bar{B} \gamma^{\mu}\left[v_{\mu}, B\right]\right)-\frac{1}{8 f^{2}} \operatorname{tr}\left(\bar{B} \gamma^{\mu}\left[\left[\Phi,\left[\Phi, v_{\mu}\right]\right], B\right]\right)+\mathcal{O}\left(\Phi^{3}\right)
\end{aligned}
$$

Analogous expressions hold for the spin-3/2 decuplet baryons.

Matrix elements of the axial-vector quark current,

$$
\mathcal{A}^{\mu a}(x)=\bar{q}(x) \gamma^{\mu} \gamma_{5} \frac{\lambda^{(a)}}{2} q(x),
$$

are obtained by taking the derivative of the baryon transition amplitude $\mathcal{F}$ with respect to the auxiliary external field $a_{\mu}$ :

$$
\left\langle\bar{p}\left|\mathcal{A}^{\mu a}(x)\right| p\right\rangle=-\left.i \frac{\delta}{\delta a_{\mu}^{a}(x)} \mathcal{F}(\bar{p}, p ; v, a, s, p)\right|_{v=0, a=0, s=\mathcal{M}_{q}, p=0} .
$$

\footnotetext{
${ }^{12}$ Kinetic and mass terms for mesons are $\mathcal{O}\left(Q^{2}\right)$.
} 
In the leading order chiral Lagrangian the external field $a_{\mu}$ is introduced via the building block $U_{\mu}$. Using (1.45) and the expansion for $U_{\mu}$ in (1.42), one derives the leading order terms in the chiral expansion of the r.h.s. of (1.48):

$$
\begin{array}{r}
\left\langle\bar{p}\left|\mathcal{A}^{\mu a}\right| p\right\rangle=\frac{1}{2}\langle\bar{p}| D \operatorname{tr}\left(\bar{B} \gamma^{\mu} \gamma_{5}\left\{\lambda^{(a)}, B\right\}\right)+F \operatorname{tr}\left(\bar{B} \gamma^{\mu} \gamma_{5}\left[\lambda^{(a)}, B\right]\right) \\
+C\left(\operatorname{tr}\left(\bar{\Delta}^{\mu} \lambda^{(a)} B\right)+\text { h.c. }\right)+H \bar{\Delta}^{\nu} \gamma^{\mu} \gamma_{5} \lambda^{(a)} \Delta_{\nu}|p\rangle
\end{array}
$$

Evaluation of (1.49) for the octet and decuplet baryon states leads directly to

$$
\begin{aligned}
\left\langle\bar{p}, \bar{\chi}, c\left|\mathcal{A}_{\mu}^{a}(0)\right| p, \chi, b\right\rangle & =\bar{u}(\bar{p}, \bar{\chi}) \gamma_{\mu} \gamma_{5} u(p, \chi)\left(D d^{a b c}+F i f^{a b c}\right) \\
\left\langle\bar{p}, \bar{\chi}, n o p\left|\mathcal{A}_{\mu}^{a}(0)\right| p, \chi, b\right\rangle & =\bar{u}_{\mu}(\bar{p}, \bar{\chi}) u(p, \chi) C \frac{1}{2 \sqrt{2}} \Lambda_{a b}^{n o p} \\
\left\langle\bar{p}, \bar{\chi}, n o p\left|\mathcal{A}_{\mu}^{a}(0)\right| p, \chi, k l m\right\rangle & =\bar{u}_{\lambda}(\bar{p}, \bar{\chi}) \gamma_{\mu} \gamma_{5} u^{\lambda}(p, \chi) H \frac{1}{2} \Lambda_{k l m}^{a, x y z} \delta_{x y z}^{n o p}
\end{aligned}
$$

with the spin- $1 / 2$ and spin- $3 / 2$ spinors $u(p, \chi)$ and $u_{\mu}(p, \chi)^{13}$, respectively, and the flavour transition tensors defined by

$$
\begin{aligned}
& \Lambda_{a b}^{k l m}=\left[\varepsilon_{i j k} \lambda_{l i}^{(a)} \lambda_{m j}^{(b)}\right]_{\mathrm{sym}(k l m)}, \quad \delta_{n o p}^{k l m}=\left[\delta_{k n} \delta_{l o} \delta_{m p}\right]_{\mathrm{sym}(n o p)}, \\
& \Lambda_{k l m}^{a b}=\left[\varepsilon_{i j k} \lambda_{i l}^{(a)} \lambda_{j m}^{(b)}\right]_{\operatorname{sym}(k l m)}, \quad \Lambda_{n o p}^{a, k l m}=\left[\lambda_{k n}^{(a)} \delta_{l o} \delta_{m p}\right]_{\operatorname{sym}(n o p)} .
\end{aligned}
$$

Here, the abbreviation $\operatorname{sym}(i j k)$ stands for the symmetrization of the indices $i, j, k$ :

$$
\operatorname{sym}(i j k)=\frac{1}{6}(i j k+\text { permutations })
$$

The flavour tensors, introduced in (1.51), are useful when dealing with different $S U(3)$ flavour contractions and will be frequently used in this work later on.

Baryon matrix elements of the axial vector currents, if coupled to the matrix elements of the leptonic weak current, govern the semi-leptonic decays of baryons, $B \rightarrow B^{\prime}+e+\bar{\nu}_{e}$. Such processes may serve to determine the parameters $F$ and $D$ in (1.45). Given the experimental values for all known semi-leptonic decays of the octet baryons, a good fit using the tree level results in (1.50) can be obtained with $D=0.80$ and $F=0.45$ (see e.g. $[14,49,52,10])^{14}$.

The value of the coupling constant $C$ can be extracted experimentally from the hadronic decays of the decuplet baryons to the octet baryons $\Delta \rightarrow N \pi, \Sigma_{[10]} \rightarrow \Sigma \pi$, etc. The

\footnotetext{
${ }^{13}$ The spinors depend on the baryon mass $M$ via the on-shell momentum $p=\left(\sqrt{M+\vec{p}^{2}}, \vec{p}\right)$ with $p^{2}=M^{2}$. See Appendix $\mathrm{H}$ for their definition and normalisation.

${ }^{14}$ One-loop corrected values for $D$ and $F$, obtained in the non-relativistic version of chiral perturbation theory, $\mathrm{HB} \chi \mathrm{PT}$, differ strongly from the tree-level result $[52,10]$. This signals a bad convergence of the strict chiral expansion. The situation is expected to be improved by partial summation of higher order terms as is most naturally done in the relativistic formulation of $\chi \mathrm{PT}$.
} 
parameter $H$ cannot be extracted from experiment directly. One-loop corrections to the axial form factors of the octet baryons or to the decuplet baryon decays provide an indirect way to determine this coupling. Estimates for $H$ obtained in one-loop HB $\chi \mathrm{PT}-$ calculations for these two, from the physical point of view very different, processes coincide within the uncertainties in both approaches $[50,15]$. Using large- $N_{c}$ sum rules, these parameters can also be correlated to the octet couplings $F$ and $D$ [21]. This correlation will also be discussed in the next chapter.

\subsection{2. $Q^{2}$ chiral Lagrangian}

Regarding the Lorentz-transformation properties of Dirac structures, the $Q^{2}$ chiral symmetry conserving interaction can be decomposed in scalar, vector, axial-vector and tensor parts:

$$
\mathcal{L}_{4-\text { point }}^{(2)}=\mathcal{L}^{(S)}+\mathcal{L}^{(V)}+\mathcal{L}^{(A)}+\mathcal{L}^{(T)} .
$$

In the following we specify these part separately. The Dirac scalar part of the interaction is given by

$$
\begin{aligned}
\mathcal{L}^{(S)} & =-\frac{1}{2} g_{0}^{(S)} \operatorname{tr}(\bar{B} B) \operatorname{tr}\left(U_{\mu} U^{\mu}\right)-\frac{1}{2} g_{1}^{(S)} \operatorname{tr}\left(\bar{B} U^{\mu}\right) \operatorname{tr}\left(U_{\mu} B\right) \\
& -\frac{1}{4} g_{D}^{(S)} \operatorname{tr}\left(\bar{B}\left\{\left\{U_{\mu}, U^{\mu}\right\}, B\right\}\right)-\frac{1}{4} g_{F}^{(S)} \operatorname{tr}\left(\bar{B}\left[\left\{U_{\mu}, U^{\mu}\right\}, B\right]\right) \\
& +\frac{1}{2} h_{1}^{(S)} \operatorname{tr}\left(\bar{\Delta}_{\mu} \cdot \Delta^{\mu}\right) \operatorname{tr}\left(U_{\nu} U^{\nu}\right)+\frac{1}{2} h_{2}^{(S)} \operatorname{tr}\left(\bar{\Delta}_{\mu} \cdot \Delta^{\nu}\right) \operatorname{tr}\left(U^{\mu} U_{\nu}\right) \\
& +h_{3}^{(S)} \operatorname{tr}\left(\left(\bar{\Delta}_{\mu} \cdot \Delta^{\mu}\right)\left(U_{\nu} U^{\nu}\right)\right)+\frac{1}{2} h_{4}^{(S)} \operatorname{tr}\left(\left(\bar{\Delta}_{\mu} \cdot \Delta^{\nu}\right)\left\{U^{\mu}, U_{\nu}\right\}\right) \\
& +h_{5}^{(S)} \operatorname{tr}\left(\left(\bar{\Delta}_{\mu} \cdot U_{\nu}\right)\left(U^{\nu} \cdot \Delta^{\mu}\right)\right) \\
& +\frac{1}{2} h_{6}^{(S)}\left(\operatorname{tr}\left(\left(\bar{\Delta}_{\mu} \cdot U^{\mu}\right)\left(U^{\nu} \cdot \Delta_{\nu}\right)\right)+\operatorname{tr}\left(\left(\bar{\Delta}_{\mu} \cdot U^{\nu}\right)\left(U^{\mu} \cdot \Delta_{\nu}\right)\right)\right) .
\end{aligned}
$$


The Dirac vector part is:

$$
\begin{aligned}
\mathcal{L}^{(V)} & =-\frac{1}{4} g_{0}^{(V)}\left(\operatorname{tr}\left(\bar{B} i \gamma^{\mu} \partial^{\nu} B\right) \operatorname{tr}\left(U_{\nu} U_{\mu}\right)+\text { h.c. }\right) \\
& -\frac{1}{8} g_{1}^{(V)}\left(\operatorname{tr}\left(\bar{B} U_{\mu}\right) i \gamma^{\mu} \operatorname{tr}\left(U_{\nu} \partial^{\nu} B\right)+\operatorname{tr}\left(\bar{B} U_{\nu}\right) i \gamma^{\mu} \operatorname{tr}\left(U_{\mu} \partial^{\nu} B\right)+\text { h.c. }\right) \\
& -\frac{1}{8} g_{D}^{(V)}\left(\operatorname{tr}\left(\bar{B} i \gamma^{\mu}\left\{\left\{U_{\mu}, U_{\nu}\right\}, \partial^{\nu} B\right\}\right)+\text { h.c. }\right) \\
& -\frac{1}{8} g_{F}^{(V)}\left(\operatorname{tr}\left(\bar{B} i \gamma^{\mu}\left[\left\{U_{\mu}, U_{\nu}\right\}, \partial^{\nu} B\right]\right)+\text { h.c. }\right) \\
& +\frac{1}{4} h_{1}^{(V)}\left(\operatorname{tr}\left(\bar{\Delta}_{\lambda} \cdot i \gamma^{\mu} \partial^{\nu} \Delta^{\lambda}\right) \operatorname{tr}\left(U_{\mu} U_{\nu}\right)+\text { h.c. }\right) \\
& +\frac{1}{4} h_{2}^{(V)}\left(\operatorname{tr}\left(\left(\bar{\Delta}_{\lambda} \cdot i \gamma^{\mu} \partial^{\nu} \Delta^{\lambda}\right)\left\{U_{\mu}, U_{\nu}\right\}\right)+\text { h.c. }\right) \\
& +\frac{1}{4} h_{3}^{(V)}\left(\operatorname{tr}\left(\left(\bar{\Delta}_{\lambda} \cdot U_{\mu}\right) i \gamma^{\mu}\left(U_{\nu} \cdot \partial^{\nu} \Delta^{\lambda}\right)\right)+\operatorname{tr}\left(\left(\bar{\Delta}_{\lambda} \cdot U_{\nu}\right) i \gamma^{\mu}\left(U_{\mu} \cdot \partial^{\nu} \Delta^{\lambda}\right)\right)+\text { h.c. }\right) .
\end{aligned}
$$

The decuplet-octet transition is described by the axial-vector part of the interaction:

$$
\begin{aligned}
\mathcal{L}^{(A)} & =-\frac{1}{4} f_{1}^{(A)}\left(\operatorname{tr}\left(\left(\bar{\Delta}^{\mu} \cdot \gamma^{\nu} \gamma_{5} B\right)\left\{U_{\mu}, U_{\nu}\right\}\right)+\text { h.c. }\right) \\
& -\frac{1}{4} f_{2}^{(A)}\left(\operatorname{tr}\left(\left(\bar{\Delta}^{\mu} \cdot \gamma^{\nu} \gamma_{5} B\right)\left[U_{\mu}, U_{\nu}\right]\right)+\text { h.c. }\right) \\
& -\frac{1}{4} f_{3}^{(A)}\left(\operatorname{tr}\left(\left(\bar{\Delta}^{\mu} \cdot U_{\nu}\right) \gamma^{\nu} \gamma_{5}\left(U_{\mu} \cdot B\right)\right)+\operatorname{tr}\left(\left(\bar{\Delta}^{\mu} \cdot U_{\mu}\right) \gamma^{\nu} \gamma_{5}\left(U_{\nu} \cdot B\right)\right)+\text { h.c. }\right) \\
& -\frac{1}{4} f_{4}^{(A)}\left(\operatorname{tr}\left(\left(\bar{\Delta}^{\mu} \cdot U_{\nu}\right) \gamma^{\nu} \gamma_{5}\left(U_{\mu} \cdot B\right)\right)-\operatorname{tr}\left(\left(\bar{\Delta}^{\mu} \cdot U_{\mu}\right) \gamma^{\nu} \gamma_{5}\left(U_{\nu} \cdot B\right)\right)+\text { h.c. }\right) .
\end{aligned}
$$

Finally, the Dirac tensor part is given by:

$$
\begin{aligned}
\mathcal{L}^{(T)} & =-\frac{1}{2} g_{1}^{(T)} \operatorname{tr}\left(\bar{B} U_{\mu}\right) i \sigma^{\mu \nu} \operatorname{tr}\left(U_{\nu} B\right)-\frac{1}{4} g_{D}^{(T)} \operatorname{tr}\left(\bar{B} i \sigma^{\mu \nu}\left\{\left[U_{\mu}, U_{\nu}\right], B\right\}\right) \\
& -\frac{1}{4} g_{F}^{(T)} \operatorname{tr}\left(\bar{B} i \sigma^{\mu \nu}\left[\left[U_{\mu}, U_{\nu}\right], B\right]\right)+\frac{1}{2} h_{1}^{(T)} \operatorname{tr}\left(\left(\bar{\Delta}_{\lambda} \cdot i \sigma^{\mu \nu} \Delta^{\lambda}\right)\left[U_{\mu}, U_{\nu}\right]\right) .
\end{aligned}
$$

Spin-3/2 fields introduce additional Lorentz structures allowing more Lorentz contractions as compared to the terms with spin- $1 / 2$ fields only. At the first sight, a lot of other contractions can be written down in $\mathcal{L}_{4-\text { point }}^{(2)}$ in terms containing the spin- $3 / 2$ fields. A closer look reveals all those terms to be vanishing, redundant or to be of higher chiral order. This can be shown with the help of the spin-3/2 constraints

$$
\gamma^{\mu} \Delta_{\mu}=\partial^{\mu} \Delta_{\mu}=0
$$

and by using partial integration and the equation of motion for baryons.

The list of $Q^{2}$ terms in the chiral Lagrangian is completed by the kinetic term for mesons and by terms describing the coupling of baryons to the external fields via the $f_{\mu \nu}^{ \pm}$tensors. We don't specify those terms since they are not relevant in this work. 
The interaction, introduced in this section, can be used to study the low-energy behavior of the product of two axial-vector currents of QCD in the baryon sector. The baryon matrix elements of this product are obtained via

$$
\left\langle\bar{p}\left|\mathcal{T} \mathcal{A}^{\mu a}(x) \mathcal{A}^{\nu b}(y)\right| p\right\rangle=-\left.\frac{\delta}{\delta a_{\mu}^{a}(x)} \frac{\delta}{\delta a_{\nu}^{b}(y)} \mathcal{F}(\bar{p}, p ; v, a, s, p)\right|_{v=0, a=0, s=M_{q}, p=0} .
$$

We discuss first the general form of the low-energy expansion of the right hand side of (1.59). Three- and four-point interaction terms, given in (1.45) and (1.53), enter the perturbative calculation of the baryon transition amplitude on the right hand side of (1.59). These terms in the chiral Lagrange density can be written as follows:

$$
\mathcal{L}(x)=F_{d c}^{a} \bar{b}^{d}(x) \gamma^{\mu} \gamma_{5} b^{c}(x) i U_{\mu}^{a}(x)+F_{d c}^{a b} \bar{b}^{d}(x) \Gamma^{\mu \nu} b^{c}(x) i U_{\mu}^{a}(x) i U_{\nu}^{b}(x) .
$$

Here, only the part of the interaction for the octet baryons is shown. Similar expressions can be written for the other vertices. The summation over different flavour structures $F_{d c}^{a}$, $F_{d c}^{a b}$ and over Cartesian flavour indices $(a, b, c, d=1, \ldots 8)$ appearing twice in a single term is implicit in (1.60). Furthermore, additional normalisation factors and chiral coupling constants are absorbed into the definition of the flavour structures in order to simplify the notation in the following. $\Gamma_{\mu \nu}$ is either the metric tensor $g_{\mu \nu}$ or an element of the Clifford algebra, eventually supplemented by an additional derivative on the baryon field in order to saturate the open Lorentz indices ${ }^{15}$.

Using (1.60), the leading order terms in the perturbative expansion of (1.59) are readily obtained:

$$
\begin{aligned}
\langle\bar{p}, \bar{\chi}, d| \mathcal{T} \mathcal{A}^{\mu a}(x) & \mathcal{A}^{\nu b}(y)|p, \chi, c\rangle \\
& =\sum_{\bar{d}=1}^{8} F_{d \bar{d}}^{a} F_{\bar{d} c}^{b} e^{i(\bar{p} x-p y)} \bar{u}(\bar{p}, \bar{\chi}) \gamma_{\mu} \gamma_{5} i S^{\bar{d}}(x-y) \gamma_{\nu} \gamma_{5} u(p, \chi) \\
& +\sum_{\bar{d}=1}^{8} F_{d \bar{d}}^{b} F_{\bar{d} c}^{a} e^{i(\bar{p} y-p x)} \bar{u}(\bar{p}, \bar{\chi}) \gamma_{\nu} \gamma_{5} i S^{\bar{d}}(y-x) \gamma_{\mu} \gamma_{5} u(p, \chi) \\
& -i e^{i(\bar{p}-p) y} \bar{u}(\bar{p}, \bar{\chi})\left(F_{d c}^{a b} \Gamma_{\mu \nu}+F_{d c}^{b a} \Gamma_{\nu \mu}\right) u(p, \chi) \delta^{4}(x-y),
\end{aligned}
$$

where the propagator for a baryon field with the flavour quantum number $d$ and the mass $M_{d}$ is given in the coordinate space by

$$
i S^{d}(x)=\int \frac{d^{4} k}{(2 \pi)^{4}} \frac{i\left(\not k+M_{d}\right)}{k^{2}-M_{d}^{2}+i \epsilon} e^{-i k x} .
$$

First two terms in (1.61) parametrise the $s$ - and $u$ - channel leading order contributions to the meson-baryon scattering and are related to each other by the interchange of the meson flavour components $a \leftrightarrow b$. The term proportional to $\delta^{4}(x-y)$ is the contact term contribution to the amplitude. These contributions can be represented by Feynman diagrams as in Figure 1.1.

\footnotetext{
${ }^{15}$ In the vertices containing $S U(3)$ decuplet baryons, the open Lorentz indices can be partially carried by spin-3/2 field operators.
} 


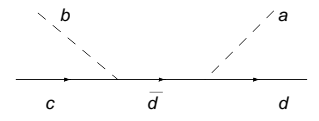

(a)

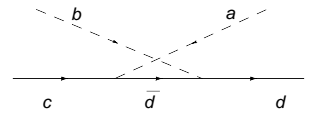

(b)

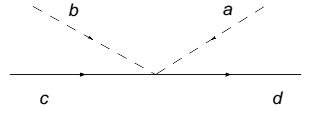

(c)

Figure 1.1.: s-, $u$-channel and contact term contribution to the meson baryon scattering.

In the following we provide an explicit form of the leading order contributions of the contact terms to the correlator

$$
C_{\mu \nu}^{a b}(q) \equiv i \int d^{4} x e^{-i q x} \mathcal{T} A_{\mu}^{a}(x) A_{\nu}^{b}(0)
$$

These contributions are obtained with the help of the $Q^{2}$-Lagrangian stated in this section. $C_{\mu \nu}^{a b}$ evaluated between the octet and decuplet leads to

$$
\begin{aligned}
&\left\langle\bar{p}, \bar{\chi}, d\left|C_{\mu \nu}^{a b}(\bar{p}-p)\right| p, \chi, c\right\rangle= \\
& \bar{u}(\bar{p}, \bar{\chi}) u(p, \chi) g_{\mu \nu} \times \frac{1}{2}\left(\left[g_{0}^{(S)}+\frac{2}{3} g_{D}^{(S)}\right] \delta_{c d} \delta_{a b}+\frac{1}{2} g_{1}^{(S)}\left[\delta_{a d} \delta_{b c}+\delta_{a c} \delta_{b d}\right]\right. \\
&\left.+g_{D}^{(S)} d_{a b e} d_{c d e}+g_{F}^{(S)} d_{a b e} i f_{c d e}\right)
\end{aligned}
$$

$$
\begin{aligned}
&\langle\bar{p}, \bar{\chi}, n o p| C_{\mu \nu}^{a b}(\bar{p}-p)|p, \chi, k l m\rangle= \\
&-\bar{u}_{\lambda}(\bar{p}, \bar{\chi}) u^{\lambda}(p, \chi) g_{\mu \nu} \times \frac{1}{4}\left(\left[h_{1}^{(S)}+\frac{2}{3} h_{3}^{(S)}+h_{5}^{(S)}\right] 2 \delta^{a b} \delta_{k l m}^{n o p}\right. \\
&\left.+\left[2 h_{3}^{(S)}+3 h_{5}^{(S)}\right] d_{a b e} \delta_{x y z}^{n o p} \Lambda_{k l m}^{e, x y z}-\frac{3}{2} h_{5}^{(S)} \delta_{r s t}^{n o p}\left(\Lambda_{x y z}^{a, r s t} \Lambda_{k l m}^{b, x y z}+\Lambda_{x y z}^{b, r s t} \Lambda_{k l m}^{a, x y z}\right)\right) \\
&-\left(\bar{u} \mu(\bar{p}, \bar{\chi}) u_{\nu}(p, \chi)+\bar{u}_{\nu}(\bar{p}, \bar{\chi}) u_{\mu}(p, \chi)\right) \times \frac{1}{8}\left(\left[h_{2}^{(S)}+\frac{2}{3} h_{4}^{(S)}+h_{6}^{(S)}\right] 2 \delta^{a b} \delta_{n o p}^{k l m}\right. \\
&\left.+\left[2 h_{4}^{(S)}+3 h_{6}^{(S)}\right] d_{a b e} \delta_{x y z}^{n o p} \Lambda_{k l m}^{e, x y z}-\frac{3}{2} h_{6}^{(S)} \delta_{r s t}^{n o p}\left(\Lambda_{x y z}^{a, r s t} \Lambda_{k l m}^{b, x y z}+\Lambda_{x y z}^{b, r s t} \Lambda_{k l m}^{a, x y z}\right)\right) \\
&-\bar{u}_{\lambda}(\bar{p}, \bar{\chi})\left(\gamma_{\mu}(p+\bar{p})_{\nu}+(p+\bar{p})_{\mu} \gamma_{\nu}\right) u^{\lambda}(p, \chi) \times \frac{1}{16}\left(\left[h_{1}^{(V)}+\frac{2}{3} h_{2}^{(V)}+h_{3}^{(V)}\right] 2 \delta^{a b} \delta_{n o p}^{k l m}\right. \\
&\left.+\left[2 h_{2}^{(V)}+3 h_{3}^{(V)}\right] d_{a b e} \delta_{x y z}^{n o p} \Lambda_{k l m}^{e, x y z}-\frac{3}{2} h_{3}^{(V)} \delta_{r s t}^{n o p}\left(\Lambda_{x y z}^{a, r s t} \Lambda_{k l m}^{b, x y z}+\Lambda_{x y z}^{b, r s t} \Lambda_{k l m}^{a, x y z}\right)\right) \\
&-\bar{u}_{\lambda}(\bar{p}, \bar{\chi}) i \sigma_{\mu \nu} u^{\lambda}(p, \chi) \times \frac{1}{2} h_{1}^{(T)} i f_{a b e} \delta_{x y z}^{n o p} \Lambda_{k l m}^{e, x y z},
\end{aligned}
$$


and

$$
\begin{aligned}
&\langle\bar{p}, \bar{\chi}, n o p C_{\mu \nu}^{a b}(\bar{p}-p)|p, \chi, c\rangle \\
&=\left(\bar{u}_{\mu}(\bar{p}, \bar{\chi}) \gamma_{\nu} \gamma_{5} u(p, \chi)+\bar{u}_{\nu}(\bar{p}, \bar{\chi}) \gamma_{\mu} \gamma_{5} u(p, \chi)\right) \\
& \quad \times \frac{1}{16 \sqrt{2}}\left(f_{1}^{(A)} 2 d_{a b e} \Lambda_{c e}^{n o p}+f_{3}^{(A)}\left[\Lambda_{a e}^{n o p}\left(d^{b c e}+i f^{b c e}\right)+\Lambda_{b e}^{\text {nop }}\left(d^{a c e}+i f^{a c e}\right)\right]\right) \\
&+\left(\bar{u}_{\mu}(\bar{p}, \bar{\chi}) \gamma_{\nu} \gamma_{5} u(p, \chi)-\bar{u}_{\nu}(\bar{p}, \bar{\chi}) \gamma_{\mu} \gamma_{5} u(p, \chi)\right) \\
& \quad \times \frac{1}{16 \sqrt{2}}\left(f_{2}^{(A)} 2 i f_{a b e} \Lambda_{c e}^{\text {nop }}+f_{4}^{(A)}\left[\Lambda_{a e}^{\text {nop }}\left(d^{b c e}+i f^{b c e}\right)-\Lambda_{b e}^{\text {nop }}\left(d^{a c e}+i f^{a c e}\right)\right]\right) .
\end{aligned}
$$

\subsubsection{Explicit symmetry breaking}

The explicit breaking of the chiral symmetry enters into the chiral Lagrangian through terms proportional to the matrices $\chi_{ \pm}$with the scalar density $s$ set equal to the quark mass matrix $\mathcal{M}_{q}$ and $p=0$. Such terms take the non-vanishment of the quark masses into account and allow a systematic expansion around the chiral limit. They give rise to the contribution to the matrix elements of the scalar and pseudo-scalar quark currents. Due to these terms the hadron masses take values different from their values in the chiral limit. In this section, terms leading and sub-leading in $\mathcal{M}_{q}$ are stated for baryons.

The symmetry breaking part of the effective Lagrange density linear in $\chi_{+}$contains $Q^{2}$ and $Q^{3}$ terms:

$$
\begin{aligned}
\mathcal{L}_{\chi}^{(2)} & =2 b_{0} \operatorname{tr}(\bar{B} B) \operatorname{tr}\left(\chi_{+}\right)+2 b_{D} \operatorname{tr}\left(\bar{B}\left\{\chi_{+}, B\right\}\right)+2 b_{F} \operatorname{tr}\left(\bar{B}\left[\chi_{+}, B\right]\right) \\
& -2 d_{0} \operatorname{tr}\left(\bar{\Delta}_{\mu} \cdot \Delta^{\mu}\right) \operatorname{tr}\left(\chi_{+}\right)-2 d_{D} \operatorname{tr}\left(\left(\bar{\Delta}_{\mu} \cdot \Delta^{\mu}\right) \chi_{+}\right), \\
\mathcal{L}_{\chi}^{(3)} & =\zeta_{0} \operatorname{tr}\left(\bar{B}\left(i \not D-\stackrel{\circ}{M}_{[8]}\right) B\right) \operatorname{tr}\left(\chi_{+}\right)+\zeta_{D} \operatorname{tr}\left(\bar{B}\left(i \not D-\stackrel{\circ}{M}_{[8]}\right)\left\{\chi_{+}, B\right\}\right) \\
& +\zeta_{F} \operatorname{tr}\left(\bar{B}\left(i \not D-\stackrel{\circ}{M}_{[8]}\right)\left[\chi_{+}, B\right]\right) \\
& -\xi_{0} \operatorname{tr}\left(\bar{\Delta}_{\mu} \cdot\left(i \not D-\stackrel{\circ}{M}_{[10]}\right) \Delta^{\mu}\right) \operatorname{tr}\left(\chi_{+}\right)-\xi_{D} \operatorname{tr}\left(\bar{\Delta}_{\mu} \cdot\left(i \not D-\stackrel{\circ}{M}_{[10]}\right) \Delta^{\mu} \chi_{+}\right) .
\end{aligned}
$$

$Q^{4}$ symmetry breaking terms are proportional to the squared matrix $\chi_{+}$:

$$
\begin{aligned}
\mathcal{L}_{\chi}^{(4)} & =c_{0} \operatorname{tr}(\bar{B} B) \operatorname{tr}\left(\chi_{+}^{2}\right)+c_{1} \operatorname{tr}\left(\bar{B} \chi_{+}\right) \operatorname{tr}\left(\chi_{+} B\right) \\
& +c_{2} \operatorname{tr}\left(\bar{B}\left\{\chi_{+}^{2}, B\right\}\right)+c_{3} \operatorname{tr}\left(\bar{B}\left[\chi_{+}^{2}, B\right]\right) \\
& +c_{4} \operatorname{tr}\left(\bar{B}\left\{\chi_{+}, B\right\}\right) \operatorname{tr}\left(\chi_{+}\right)+c_{5} \operatorname{tr}\left(\bar{B}\left[\chi_{+}, B\right]\right) \operatorname{tr}\left(\chi_{+}\right) \\
& +c_{6} \operatorname{tr}(\bar{B} B)\left(\operatorname{tr}\left(\chi_{+}\right)\right)^{2} \\
& -e_{0} \operatorname{tr}\left(\bar{\Delta}_{\mu} \cdot \Delta^{\mu}\right) \operatorname{tr}\left(\chi_{+}^{2}\right)-e_{1} \operatorname{tr}\left(\left(\bar{\Delta}_{\mu} \cdot \chi_{+}\right)\left(\chi_{+} \cdot \Delta^{\mu}\right)\right) \\
& -e_{2} \operatorname{tr}\left(\left(\bar{\Delta}_{\mu} \cdot \Delta^{\mu}\right) \cdot \chi_{+}^{2}\right)-e_{3} \operatorname{tr}\left(\left(\bar{\Delta}_{\mu} \cdot \Delta^{\mu}\right) \cdot \chi_{+}\right) \operatorname{tr}\left(\chi_{+}\right) \\
& -e_{4} \operatorname{tr}\left(\bar{\Delta}_{\mu} \cdot \Delta^{\mu}\right)\left(\operatorname{tr}\left(\chi_{+}\right)\right)^{2} .
\end{aligned}
$$


The baryon mass shifts up to the fourth chiral order, caused by the symmetry breaking part of the chiral Lagrangian, are readily obtained by evaluating the tree-level baryon self energies with $(1.67-1.69)$. We assume the perfect isospin symmetry throughout this work and write

$$
m_{u}=m_{d} \equiv m .
$$

Upon expanding the matrix $\chi_{+}$as in (1.42) and retaining only relevant terms in this expansion, the explicit expressions for the self energies of the octet baryons read

$$
\begin{aligned}
& \Sigma_{N}^{\text {tree-level }}=-4 B_{0}\left(b_{0}^{\text {eff }}\left(2 m+m_{s}\right)+b_{D}^{\text {eff }}\left(m+m_{s}\right)+b_{F}^{\text {eff }}\left(m-m_{s}\right)\right) \\
& -4 B_{0}^{2}\left(c_{0}\left(2 m^{2}+m_{s}^{2}\right)+c_{2}\left(m^{2}+m_{s}^{2}\right)+c_{3}\left(m^{2}-m_{s}^{2}\right)\right) \\
& -2 B_{0}\left(\zeta_{0}\left(2 m+m_{s}\right)+\zeta_{D}\left(m+m_{s}\right)+\zeta_{F}\left(m-m_{s}\right)\right)\left(M_{N}-\stackrel{\circ}{M}_{[8]}\right) \text {, } \\
& \Sigma_{\Lambda}^{\text {tree-level }}=-4 B_{0}\left(b_{0}^{\text {eff }}\left(2 m+m_{s}\right)+\frac{2}{3} b_{D}^{\text {eff }}\left(m+2 m_{s}\right)\right) \\
& -4 B_{0}^{2}\left(c_{0}\left(2 m^{2}+m_{s}^{2}\right)+\frac{2}{3} c_{1}\left(m-m_{s}\right)^{2}+\frac{2}{3} c_{2}\left(m^{2}+2 m_{s}^{2}\right)\right) \\
& -2 B_{0}\left(\zeta_{0}\left(2 m+m_{s}\right)+\frac{2}{3} \zeta_{D}\left(m+2 m_{s}\right)\right)\left(M_{\Lambda}-\stackrel{\circ}{M}_{[8]}\right), \\
& \Sigma_{\Sigma}^{\text {tree-level }}=-4 B_{0}\left(b_{0}^{\text {eff }}\left(2 m+m_{s}\right)+2 b_{D}^{\text {eff }} m\right)-4 B_{0}^{2}\left(c_{0}\left(2 m^{2}+m_{s}^{2}\right)+2 c_{2} m^{2}\right) \\
& -2 B_{0}\left(\zeta_{0}\left(2 m+m_{s}\right)+2 \zeta_{D} m\right)\left(M_{\Sigma}-\stackrel{\circ}{M}[8]\right), \\
& \Sigma_{\Xi}^{\text {tree-level }}=-4 B_{0}\left(b_{0}^{\text {eff }}\left(2 m+m_{s}\right)+b_{D}^{\text {eff }}\left(m+m_{s}\right)-b_{F}^{\text {eff }}\left(m-m_{s}\right)\right) \\
& -4 B_{0}^{2}\left(c_{0}\left(2 m^{2}+m_{s}^{2}\right)+c_{2}\left(m^{2}+m_{s}^{2}\right)-c_{3}\left(m^{2}-m_{s}^{2}\right)\right) \\
& -2 B_{0}\left(\zeta_{0}\left(2 m+m_{s}\right)+\zeta_{D}\left(m+m_{s}\right)-\zeta_{F}\left(m-m_{s}\right)\right)\left(M_{\Xi}-\stackrel{\circ}{M}_{[8]}\right) \text {, } \\
& \Sigma_{\Delta}^{\text {tree-level }}=-4 B_{0}\left(d_{0}^{\text {eff }}\left(2 m+m_{s}\right)+d_{D}^{\text {eff }} m\right)-4 B_{0}^{2}\left(e_{0}\left(2 m^{2}+m_{s}^{2}\right)+e_{2} m^{2}\right) \\
& -2 B_{0}\left(\xi_{0}\left(2 m+m_{s}\right)+\xi_{D} m\right)\left(M_{\Delta}-\stackrel{\circ}{M}_{[10]}\right) \text {, } \\
& \Sigma_{\Sigma^{*}}^{\text {tree-level }}=-4 B_{0}\left(d_{0}^{\text {eff }}\left(2 m+m_{s}\right)+\frac{1}{3} d_{D}^{\text {eff }}\left(2 m+m_{s}\right)\right) \\
& -4 B_{0}^{2}\left(e_{0}\left(2 m^{2}+m_{s}^{2}\right)+\frac{1}{3} e_{1}\left(m-m_{s}\right)^{2}+\frac{1}{3} e_{2}\left(2 m^{2}+m_{s}^{2}\right)\right) \\
& -2 B_{0}\left(\xi_{0}\left(2 m+m_{s}\right)+\frac{1}{3} \xi_{D}\left(2 m+m_{s}\right)\right)\left(M_{\Sigma^{*}}-\stackrel{\circ}{M}_{[10]}\right), \\
& \Sigma_{\Xi^{*}}^{\text {tree-level }}=-4 B_{0}\left(d_{0}^{\text {eff }}\left(2 m+m_{s}\right)+\frac{1}{3} d_{D}^{\text {eff }}\left(m+2 m_{s}\right)\right) \\
& -4 B_{0}^{2}\left(e_{0}\left(2 m^{2}+m_{s}^{2}\right)+\frac{1}{3} e_{1}\left(m-m_{s}\right)^{2}+\frac{1}{3} e_{2}\left(m^{2}+2 m_{s}^{2}\right)\right), \\
& -2 B_{0}\left(\xi_{0}\left(2 m+m_{s}\right)+\frac{1}{3} \xi_{D}\left(m+2 m_{s}\right)\right)\left(M_{\Xi^{*}}-\stackrel{\circ}{M}[10]\right), \\
& \Sigma_{\Omega}^{\text {tree-level }}=-4 B_{0}\left(d_{0}^{\text {eff }}\left(2 m+m_{s}\right)+d_{D}^{\text {eff }} m_{s}\right)-4 B_{0}\left(e_{0}\left(2 m^{2}+m_{s}^{2}\right)+e_{2} m_{s}^{2}\right)
\end{aligned}
$$




$$
-2 B_{0}\left(\xi_{0}\left(2 m+m_{s}\right)+\xi_{D} m_{s}\right)\left(M_{\Omega}-\stackrel{\circ}{M}_{[10]}\right),
$$

with

$$
\begin{array}{ll}
b_{0}^{\mathrm{eff}}=b_{0}+c_{6} B_{0}\left(2 m+m_{s}\right), & b_{D}^{\mathrm{eff}}=b_{D}+c_{4} B_{0}\left(2 m+m_{s}\right), \\
b_{F}^{\mathrm{eff}}=b_{F}+c_{5} B_{0}\left(2 m+m_{s}\right), & \\
d_{0}^{\mathrm{eff}}=d_{0}+e_{4} B_{0}\left(2 m+m_{s}\right), & d_{D}^{\mathrm{eff}}=d_{D}+e_{3} B_{0}\left(2 m+m_{s}\right) .
\end{array}
$$

Terms in (1.68) ask for an additional remark. Using the equation of motion for the baryons fields, the components in (1.68) can be eliminated. Therefore, in the presence of the complete chiral Lagrangian, the interaction part contained in $\mathcal{L}_{\chi}^{(3)}$ is redundant. Nevertheless, this part may turn to be relevant, if the chiral expansion is truncated at some order and the partial effect of higher order terms contained in $\mathcal{L}_{\chi}^{(3)}$ needs to be considered. One example for such situation is the chiral expansion of the baryon masses and its extrapolation to higher quark masses. This will be discussed in Section 3.1 in more details. Here, we consider the $Q^{4}$ effect of the terms in $\mathcal{L}_{\chi}^{(3)}$, which can be completely absorbed into the redefinition of the couplings in $\mathcal{L}_{\chi}^{(4)}$.

With the help of the equation of motion, the $Q^{4}$ effects of $\mathcal{L}_{\chi}^{(3)}$ are disentangled from the residual effects $\left(Q^{6}\right)$ via the following decomposition

$$
\begin{aligned}
c_{0} & =2\left(\zeta_{D} b_{D}-\zeta_{F} b_{F}\right)+c_{0}^{\chi}, & e_{0} & =e_{0}^{\chi}, \\
c_{1} & =\frac{4}{3}\left(\zeta_{D} b_{D}-3 \zeta_{F} b_{F}\right)+c_{1}^{\chi}, & e_{1} & =-\frac{4}{3} \xi_{D} d_{D}+e_{1}^{\chi}, \\
c_{2} & =-2\left(\zeta_{D} b_{D}-3 \zeta_{F} b_{F}\right)+c_{2}^{\chi}, & e_{2} & =2 \xi_{D} d_{D}+e_{2}^{\chi}, \\
c_{3} & =2\left(\zeta_{D} b_{F}+\zeta_{F} b_{D}\right)+c_{3}^{\chi}, & e_{3} & =2\left(\xi_{0} d_{D}+\xi_{D} d_{0}\right)+e_{3}^{\chi}, \\
c_{4} & =2\left(\zeta_{0} b_{D}+\zeta_{D} b_{0}+2 \zeta_{D} b_{D}-2 \zeta_{F} b_{F}\right)+c_{4}^{\chi}, & & e_{4}=2 \xi_{0} d_{0}+e_{4}^{\chi}, \\
c_{5} & =2\left(\zeta_{0} b_{F}+\zeta_{F} b_{0}\right)+c_{5}^{\chi}, & & \\
c_{6} & =2\left(\zeta_{0} b_{0}-\zeta_{D} b_{D}+\zeta_{F} b_{F}\right)+c_{6}^{\chi} . & &
\end{aligned}
$$

which implies that a chiral decomposition of the baryon masses in terms of the $c_{i}^{\chi}$ and $e_{i}^{\chi}$ parameters does not depend on $\zeta_{0}, \zeta_{D}, \zeta_{F}$ and $\xi_{0}, \xi_{D}$, if truncated at order $Q^{4}$. 


\section{Chapter 2.}

\section{QCD in the large- $N_{c}$ limit}

As already mentioned in the introduction, the formulation of QCD in the limit of an infinite number of colours provides a powerful approach to the strong interaction. The aim of this chapter will not be to give a comprehensive overview of this framework. The presentation in the next three sections will be rather targeted directly on the methods used for the description of the meson-baryon interaction and the static properties of the baryons in the large- $N_{c}$ limit. This knowledge allows to match the description of the interaction in both frameworks - EFT for mesons and baryons and QCD in the limit of large $N_{c}$ - and will provide several constraints for the coupling constants in the chiral Lagrangian.

In the next three sections it will be tried to give a closed and consistent overview of these methods. The last section contains some applications and shows the interplay of both approaches. The large- $N_{c}$ constraints for the chiral Lagrangian are worked out there.

There is extensive treatment of this formalism in the literature on the introductional level $([18,96,68,67,85])$. Some of the references to the original literature will be cited here during the development of the text. References to the works on the topics, that are not touched here, can be taken from the introductional texts mentioned above.

\subsection{Introduction}

QCD is readily generalised for arbitrary number of colours - the local gauge group is promoted to $U\left(N_{c}\right)^{1}$. A typical diagram in a non-abelian theory is a gauge boson vacuum polarisation, shown in Figure 2.1(a). After the summation over all possible colours in the loop is done, this diagram picks up an overall factor $N_{c}$. In order to make such class of diagrams well behaving in the limit $N_{c} \rightarrow \infty$, the product $g^{2} N_{c}$ needs to be kept fixed in this limit. This suggests a rescaling of the coupling constant:

$$
g \rightarrow \frac{g}{\sqrt{N_{c}}} .
$$

\footnotetext{
${ }^{1}$ The difference between $S U(N)$ and $U(N)$ in not relevant in the limit $N_{c} \rightarrow \infty$. In fact, the double line notation, to be described below, is, strictly speaking, valid for $U(N)$ only.
} 


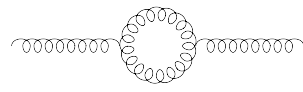

(a)

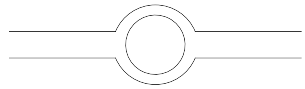

(b)

Figure 2.1.: Gluon propagator with gluon loop in usual and in double line notation.

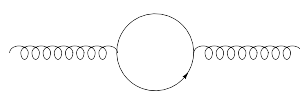

(a)

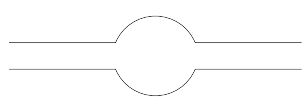

(b)

Figure 2.2.: Gluon propagator with quark loop in usual and in double line notation.

Therefore, in order to determine the overall $N_{c}$-factor of an arbitrary diagram one needs to count the closed colour loops, each of them producing a factor $N_{c}$, and the explicit occurrences of the coupling constant $g$ (factor $1 / \sqrt{N_{c}}$ ).

The number of closed colour loops is more economically obtained by using t'Hooft's double line notation, where the gluon line is visualised in the diagrams by a quark-antiquark pair [82]. Using the double-line notation, a closed colour loop corresponds to a closed quarkantiquark loop.

The gluon propagator with gluon- and quark-loop insertions is shown in the usual and in the double-line notations in Figures 2.1 and 2.2, respectively. The diagram with the virtual gluon loop involves two vertices and a closed colour loop. Its scaling is:

$$
\text { Figure } 2.1 \sim g^{2} N_{c} \sim\left(\frac{1}{\sqrt{N_{c}}}\right)^{2} N_{c}=1
$$

On the other hand, a virtual quark loop is not accompanied by a closed colour loop

$$
\text { Figure } 2.2 \sim g^{2} \sim\left(\frac{1}{\sqrt{N_{c}}}\right)^{2}=\frac{1}{N_{c}} \text {, }
$$

and is suppressed therefore by a factor $1 / N_{c}$ as compared to diagrams without virtual quark loops ${ }^{2}$. Having this, a carefull study of the $N_{c}$ counting in the diagrams leads to the conclusion that only a certain class of diagrams survives in the limit $N_{c} \rightarrow \infty$. These are the so-called planar diagrams which can be embedded in a single plane without having two propagators crossing each other, except at a vertex. The leading diagrams are planar diagrams where the single quark loop, if it exists, builds the edge of the diagram. Further internal quark loops are suppressed.

\footnotetext{
${ }^{2}$ One distinguishes between the 't Hooft limit, where the number of quark flavours is small and fixed and the Venenziano limit, where the the ratio $N_{F} / N_{c}$ is hold fixed as $N_{c} \rightarrow \infty$. An internal quark loop, containing the sum over all possible flavours, is not suppressed in the later case.
} 


\subsection{Meson and baryon couplings}

We sketch briefly the derivation of the scaling behaviour of the meson decay constants, which helps to become familiar with the $N_{c}$ counting in the diagrams. Consider first the quark bilinear $J=\bar{q} \Gamma q$. Choosing the appropriate Dirac-structure $\Gamma$, such a bilinear serves as an interpolating field for a meson. The leading order digram for the meson two-point function is shown in Figure 2.3(a). It is of the order $N_{c}$ because of the quark loop, which, as already mentioned, contains a sum over colour degrees of freedom. All the other planar diagrams with insertions of an arbitrary number of gluon lines are of the same order. An important observation of Witten in [95] was that in the limit $N_{c} \rightarrow \infty$, the quark bilinear $J$ interpolates between a one-meson state and vacuum only. That is, cutting of any planar diagram at an arbitrary place, the only colour-singlet contractions of quark and gluons one discovers are $\bar{q} q$-mesons - there are no multi-particle, no glue, no hybrid states at leading order in $N_{c}$. Thus, the spectral representation of $\langle 0|J(x) J(y)| 0\rangle$ is

$$
\langle 0|J(x) J(y)| 0\rangle=\sum_{n}\langle 0|J(x)| n\rangle\langle n|J(y)| 0\rangle=\sum_{n} e^{-i p_{n}(x-y)}|\langle 0|J(0)| n\rangle|^{2}=\mathcal{O}\left(N_{c}\right),
$$

where the sum runs over (continuous or discrete) single-meson states only. Since the intermidiate states doesn't have any $N_{c}$-dependence, the scaling behaviour of $\langle 0|J(x) J(y)| 0\rangle$ is fully determined by $|\langle 0|J(0)| n\rangle|^{2}$. From this we have for the amplitude to create a meson from the vacuum

$$
Z_{n}=|\langle 0|J(0)| n\rangle|^{2}=\mathcal{O}\left(N_{c}\right),
$$

and for the meson decay constant

$$
f_{n} \sim\langle 0|J(0)| n\rangle=\mathcal{O}\left(\sqrt{N_{c}}\right) .
$$

The $N_{c}$ behaviour of the scattering amplitude of $n$ mesons $m_{i}(i=1, \ldots, n)$ can be deduced from the LSZ reduction formula:

$$
\mathcal{M}_{m_{1} \ldots m_{k} \rightarrow m_{k+1} \ldots m_{n}} \sim \frac{1}{(\sqrt{Z})^{n}}\left\langle 0\left|\mathcal{T} J\left(x_{1}\right) \ldots J\left(x_{n}\right)\right| 0\right\rangle \sim \frac{1}{\sqrt{N_{c}}{ }^{n}} N_{c}=N_{c}^{1-n / 2},
$$

where the factor $N_{c}$ stems from the quark loop of the $n$-point function (see Figure 2.3(b)).

To derive the $N_{c}$-counting for graphs with baryons, consider first the meson-quark coupling. The leading diagram is shown in Figure 2.4(a). This vertex scales as $1 / \sqrt{N_{c}}$. To build a baryonic state one needs to combine $N_{c}$ quarks with the help of the $S U(N) \varepsilon$ tensor into a colourless antisymmetric state. So, the generalisation for the meson-baryon vertex is simple:

$$
\text { meson-baryon vertex } \sim \frac{1}{\sqrt{N_{c}}} N_{c}=\sqrt{N_{c}} \text {. }
$$




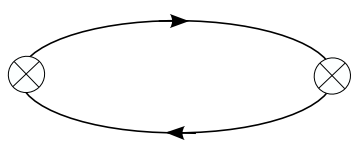

(a) Two-point function

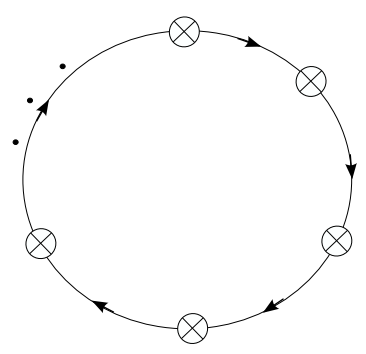

(b) $n$-point function

Figure 2.3.: A meson-meson correlation functions.

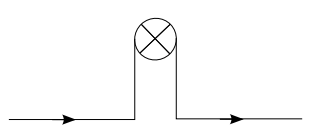

(a) single quark

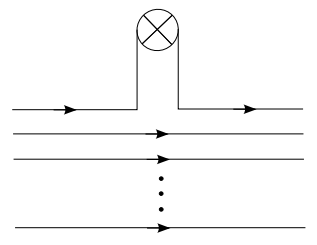

(b) $N_{c}$ quarks

Figure 2.4.: Quark-meson and baryon-meson vertices.

Multiplication with $N_{c}$ in (2.8) reflects the freedom of choice of one of the $N_{c}$ quarks in the baryon (Figure 2.4(b)).

Similar to the meson-meson scattering, the corresponding $N_{c^{-}}$scaling for the meson-baryon scattering amplitude is read off

$$
\mathcal{M}_{B m_{1} \ldots m_{k} \rightarrow B^{\prime} m_{k+1} \ldots m_{n}} \sim \frac{1}{(\sqrt{Z})^{n}}\left\langle\mathcal{B}^{\prime}\left|\mathcal{T} J\left(x_{1}\right) \ldots J\left(x_{n}\right)\right| \mathcal{B}\right\rangle \sim \frac{1}{{\sqrt{N_{c}}}^{n}} N_{c}=N_{c}^{1-n / 2}
$$

Again, we have a factor $1 / \sqrt{N_{c}}$ for each creation/absorption of a meson and a factor $N_{c}$ for $N_{c}$ possibilities to choose a quark line. Placing a pair of meson lines on two different quark lines would rise the combinatorial factor to $N_{c}\left(N_{c}-1\right)$ but would also require an additional gluon exchange, which brings in a factor $1 / N_{c}$. Also in this case the overall scaling of the amplitude is given by (2.9).

\subsection{Baryons in $1 / N_{c}$-expansion}

The diagrammatic study of the baryons in the $1 / N_{c}$ expansion is complicated by the fact, that one needs to take $N_{c}$ quark lines into account which lead to combinatorial factors. In [95] Witten splitted the problem of describing the properties of baryons in two parts, first to determine the interaction of quarks in large- $N_{c}$ limit by using graphical/combinatorial methods, and then to use this interaction together with the many-body techniques applied to $N_{c}$-body states. The $n$-quark interaction is $\mathcal{O}\left(N_{c}^{1-n}\right)$ and the baryon mass is $\mathcal{O}\left(N_{c}\right)$. 
This is reminiscent of the soliton behaviour in non-linear theories. Witten suggested to identify the baryons in the large- $N_{c}$ limit with the solitons of the Skyrme model. The results obtained in [95] are based on the Hartree-approximation, which become exact in the case of infinite densities and of vanishing interparticle interactions.

In the following we want to introduce an algebraic approach, where the study of baryons is lead back to the study of spin and flavour properties of baryons in the large- $N_{c}$ limit by means of (auxiliary) spin-flavour quark operators describing static quarks. These static quark operators are similar to those used used in nonrelativistic constituent quark models and are closely connected to the quark-gluon dynamics of QCD. Furthermore, the expansion of baryon properties in inverse powers of $N_{c}$ is related to the diagrammatic expansion of matrix elements of various current quark operators in the interaction picture. This algebraic approach allows also to study baryons for finite values of $N_{c}$ systematically.

\subsubsection{Matrix elements of baryon operators}

This section is mainly based on the work of Luty and March-Russell in [63]. We refer to the original work for some of the subtleties omitted here.

The main idea behind the approach of [63] is based on the description of the ground state baryons in terms of localised quarks. Quarks are put in a coordinate dependent scalar potential wall. The average field seen by every quark is constructed in such a way as to make localised quark states possible. This can be achieved by introducing a position dependent quark mass matrix $\mathcal{M}_{q}(|\vec{x}|)$. The full QCD Hamilton operator, $H$, may be split into a mean-field part, $H_{0}$, and the interaction part, $V$ :

$$
H_{Q C D}=H_{0}+V
$$

without changing the structure of $H$. This procedure, which should be regarded just as a mathematical trick, reorganises the perturbative expansion and helps to study the structure of matrix elements in the large- $N_{c}$ limit.

The "free" field operator, $\psi_{I}(t, \vec{x})$, describing quarks in the mean scalar field, can be expanded in the energy eigenmodes of the $H_{0}$ as follows:

$$
\psi_{I}(t, \vec{x})=\sum_{n=0}^{\infty} \sum_{a=1}^{2}\left(u_{n, a}(\vec{x}) e^{-i E_{n} t} b_{n, a}+v_{n, a}(\vec{x}) e^{+i E_{n} t} d_{n, a}^{\dagger}\right) .
$$

Here, $b_{n, a}$ destroys a quark in the $n$th mode with the spin quantum number $a$ and $d_{n, a}^{\dagger}$ creates an anti-quark with the quantum numbers $n$ and $a . E_{n}$ is the corresponding energy eigenvalue. The objects of main interest are the baryonic ground states which are the lowest colour-neutral eigenstates of the Hamilton operator $H_{0}$ in (2.10),

$$
\left|\mathcal{B}_{0}\right\rangle=\mathcal{B}^{\alpha_{1} a_{1} \ldots \alpha_{N_{c}} a_{N_{c}}} \varepsilon^{A_{1} \ldots A_{N_{c}}} b_{0, \alpha_{1} a_{1} A_{1}}^{\dagger} \cdots b_{0, \alpha_{N_{c}} a_{N_{c}} A_{N_{c}}}^{\dagger}|0\rangle,
$$

with the spin indices $\alpha_{i}=1,2$, the flavour indices $a_{i}=1, \ldots N_{F}$ and with the colour indices $A_{i}=1, \ldots N_{c}$. The colour structure of these baryonic states is completely furnished by the antisymmetric tensor $\varepsilon$, whereas the "wave function" $\mathcal{B}$ specifies the spin 
and the flavour quantum numbers. The state $\left|\mathcal{B}_{0}\right\rangle$ is a tensor product of $N_{c}$ one-quark states, created by the quark creation operators $b_{0, \alpha a A}^{\dagger}$ acting on the ground state of $H_{0}$, $|0\rangle$. It is always possible to adjust the scalar mean field discussed above in such a way as to make the lightest mode component describing a quark state localised on the scale of typical hadron size.

The interacting quark field operator in the Heisenber picture is connected to its representation in the interaction picture in $(2.11)$ by a unitary transformation ${ }^{3}$ :

$$
\psi(t, \vec{x})=U^{\dagger}(t) \psi_{I}(t, \vec{x}) U(t), \quad U(t)=e^{+i H_{0} t} e^{-i H t}=\mathcal{T} \exp \left[-i \int_{0}^{t} d t^{\prime} V_{I}\left(t^{\prime}\right)\right]
$$

with $V_{I}(t)=e^{i H_{0} t} V e^{-i H t}$. The states $\left|\mathcal{B}_{0}\right\rangle$ evolve to the eigenstates of the full Hamiltonian, $|\mathcal{B}\rangle$, with the energy $E_{\mathcal{B}}$ according to

$$
e^{-i H t}\left|\mathcal{B}_{0}\right\rangle=|\mathcal{B}\rangle\left\langle\mathcal{B} \mid \mathcal{B}_{0}\right\rangle e^{-i E_{\mathcal{B}} t}+\ldots
$$

The omitted terms, denoted by ellipses, are exponentially suppressed for $t \rightarrow \infty(1-i \varepsilon)$. The states $|\mathcal{B}\rangle$ still have the quantum numbers of the states $\left|\mathcal{B}_{0}\right\rangle$, in particular the baryon number one, and are the ground states of $H$. These states are identified with the onebaryon states. All the other states with the same quantum numbers as $\left|\mathcal{B}_{0}\right\rangle$ (additional inclusion of colour-flavour singlet mesonic, gluonic and exotic degrees of freedom) would result in higher energy states.

With the help of (2.14) the calculation of the matrix elements of arbitrary Heisenberg quark operators $\hat{\mathcal{O}}_{i}\left(x_{i}\right)$ (or products of them) involving the states $|\mathcal{B}\rangle$ can be reduced to the calculation using the ground states of the unperturbed Hamiltonian and the corresponding operators in the interaction picture, $\mathcal{O}_{I i}\left(x_{i}\right)$ :

$$
\begin{aligned}
& \left\langle\mathcal{B}^{\prime}\left|\mathcal{T} \hat{\mathcal{O}}_{1}\left(x_{1}\right) \ldots \hat{\mathcal{O}}_{n}\left(x_{n}\right)\right| \mathcal{B}\right\rangle=\frac{\left\langle\mathcal{B}_{0}^{\prime}\left|\hat{\mathcal{O}}_{I 1}\left(x_{1}\right) \ldots \hat{\mathcal{O}}_{I n}\left(x_{n}\right) U_{I}\right| \mathcal{B}_{0}\right\rangle}{\left\langle\mathcal{B}_{0}^{\prime}\left|U_{I}\right| \mathcal{B}_{0}^{\prime}\right\rangle^{1 / 2}\left\langle\mathcal{B}_{0}\left|U_{I}\right| \mathcal{B}_{0}\right\rangle^{1 / 2}} \\
& U(-T)|\mathcal{B}\rangle=\frac{\left|\mathcal{B}_{0}\right\rangle}{\left\langle\mathcal{B}_{0}\left|U_{I}\right| \mathcal{B}_{0}\right\rangle^{1 / 2}}+\cdots, \quad U_{I}=\mathcal{T} \exp \left[-i \int_{-T}^{+T} d t^{\prime} V_{I}\left(t^{\prime}\right)\right]
\end{aligned}
$$

for sufficiently large $T$ and $t_{1}, \ldots t_{n}$ lying in the interval $(-T, T)$. This formula resembles the result of the Gell-Man-Low theorem for vacuum field theories. Here, the state $\left|\mathcal{B}_{0}\right\rangle$ plays the same role as the unperturbed vacuum state of a free field theory. The denominator serves to cancel the phase factors in (2.14).

As in vacuum field theory, the r.h.s. of this exact relation can be calculated in a perturbative way by using a small adaptation of Feynman rules. The time ordered product is written as a sum of all contractions and products of normal ordered operators. The

\footnotetext{
${ }^{3}$ Connection to the familiar textbook representation on quantum field theory is clear. In the LSZ formalism the transition to the interacting fields is done starting from the asymptotic free fields. These in- and out-fields are given by the plane wave solutions of the equation of motion corresponding to the free Hamilton operator of the theory. Here, the role of the asymptotic fields is taken over by the fields in (2.11) and the transition to the full interacting fields is done with respect to $V$ in $(2.10)$.
} 


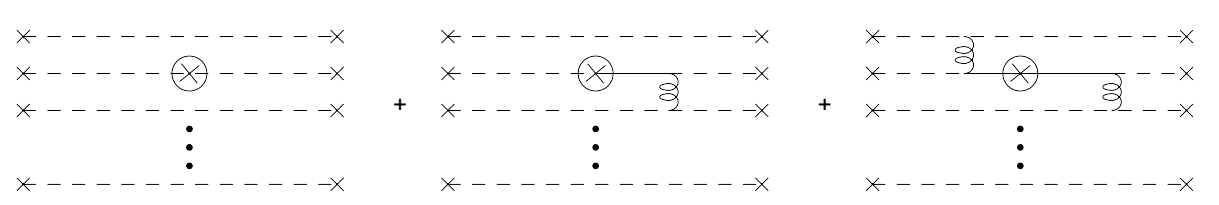

(a) Expansion in terms of quark-gluon vertices

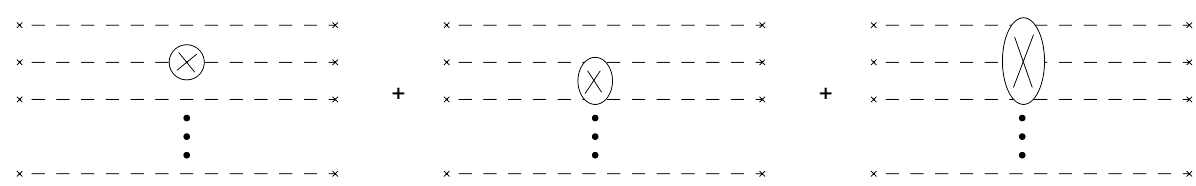

(b) Expansion in terms of effective vertices

Figure 2.5.: Diagrammatic representation of the matrix elements of an one-body operator in $(2.15)$.

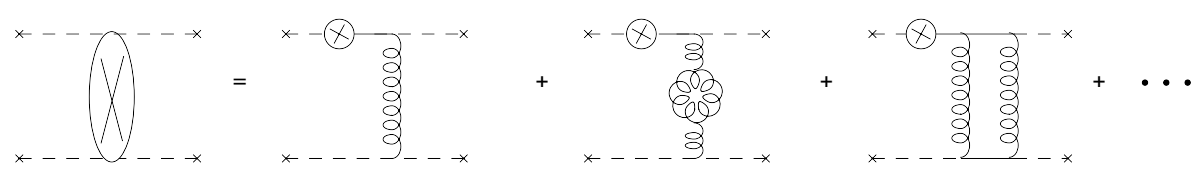

Figure 2.6.: Diagrammatic representation of a two-body operator.

denominator in $(2.15)$ cancels those terms in the expansion, where all the operators are contracted $^{4}$. In contrast to the vacuum case, normal ordered products can also contribute, because the operators are evaluated between non-vacuum states. Upon normal ordering, only the ground state modes in (2.11) can contribute if evaluated between the baryon ground states in (2.12). These contributions are represented diagrammatically by $N_{c}$ inand out-going dashed lines. In contrast to them, the fermion contractions $\left\{\psi_{I}(x), \bar{\psi}_{I}(y)\right\}$ at the inner lines contain contributions from all modes in (2.11) and are represented by solid lines.

Although it is not clear, whether the expansion in the strong coupling $g_{s}$ in $(2.15)$ converges or not, it is still possible to determine the relevant contributions in the large- $N_{c}$ limit. The diagrammatic expansion of the r.h.s. of (2.15) is shown in Figure 2.5(a) for a single quark operator $\hat{\mathcal{O}}(x)=\bar{\psi}(x) \Gamma \psi(x)$ with an arbitrary Dirac flavour matrix $\Gamma$. The insertion of $\hat{\mathcal{O}}_{I}(x)$ on a quark line and additional gluon exchanges, connecting several dashed quark lines, can be thought of as being "effective vertices". Such building blocks, consisting of $r$ 0th-mode quark creation and annihilation operators, are called $r$-body operators and are denoted in the following by $\hat{\mathcal{O}}^{(r)}$.

The expansion in terms of such effective operators is indicated in Figure 2.5(b). A two-body operator connects two dashed quark lines and is generated by insertion of an one-body operator and at least one additional gluon exchange (see Figure 2.6). Similar, a three-body operator connects three lines and is generated by insertion of an one-body operator and at least two gluon exchanges, connecting two different quark lines and so

\footnotetext{
${ }^{4}$ This statement is not so obvious as in the vacuum case, where all the vacuum diagrams exponentiate and are canceled by the corresponding denominator in (2.15). See [63] for details.
} 
on.

In general, the sum of all diagrams representing the r.h.s. of (2.15) can be written as a sum over $r$-body operators, evaluated between baryon states:

$$
\left\langle\mathcal{B}^{\prime}\left|\mathcal{T} \hat{\mathcal{O}}_{1}\left(x_{1}\right) \ldots \hat{\mathcal{O}}_{n}\left(x_{n}\right)\right| \mathcal{B}\right\rangle=\sum_{l} \sum_{r=1}^{N_{c}} c_{r}^{l}\left(x_{1}, \ldots, x_{n}\right)\left\langle\mathcal{B}_{0}^{\prime}\left|\hat{\mathcal{O}}_{l}^{(r)}\right| \mathcal{B}_{0}\right\rangle
$$

Here, the first summation is done over all $r$-body operators. Since a baryon consists of $N_{c}$ quarks, this sum terminates at $r=N_{c}$. In the most cases of interest, the Heisenberg quark operators are colourless. Thus, the operators on the both sides of (2.16) are specified by spin and flavour indices only and transform according to some irreducible representation of the flavour symmetry group and of the group of spatial rotations in the baryon rest frame, $S U\left(N_{F}\right) \otimes S U(2)$. Given $r$, the summation over all possible representations $l$ is done. The $N_{c}$-scaling of the unknown functions $c_{r}^{l}$, which parametrise the complicated structure of quark-gluon diagrams, is given by

$$
c_{r}^{l} \sim \frac{1}{N_{c}^{r-1+L}},
$$

where $L$ is the minimal number of quark loops in the diagrams contributing to the matrix elements. The factor $1 / N_{c}^{r-1}$, which accompanies each $r$-body operator in the expansion in (2.16) is due to the fact that one needs at least $r-1$ gluon exchanges on the quarkgluon-level to generate an $r$-body operator as illustrated in Figure (2.5). Furthermore, the factor $1 / N_{c}^{L}$ accounts for the already mentioned suppression of quark loops on the inner lines. Contribution of an $r$-body operator, when evaluated on the different quark lines, can cancel each other. Thus, (2.17) provides an upper bound for the scaling behaviour of the coefficient functions.

Clearly, the spin-flavour structure on both sides in (2.16) should match. Depending on the particular features of the matrix elements under consideration, the coefficients $c_{r}^{l}$ can also carry open Lorentz indices and transform under some representation of $S U(2)$. In this case the spin-flavour structure of the operators $\hat{\mathcal{O}}_{l}^{(r)}$ alone doesn't match to the structure of the product of operators on the l.h.s. of (2.16).

In dealing with colourless operators acting on colourless baryonic states, the colour indices may be omitted ${ }^{5}$. This helps to simplify the calculation of the matrix elements of the effective operators. Since a multiparticle fermionic state in (2.12) is antisymmetric with respect to the interchange of two particles, omitting of the colour indices results in a totally symmetric spin-flavour state. The Fock space of such states is created with the help of creation and annihilation operators, which keep track of spin and flavour indices only. These operators, denoted by $q_{\alpha a}^{\dagger}$ and $q^{\alpha a}$, obey the bosonic commutator algebra:

$$
\left[q^{\alpha a}, q_{\beta b}^{\dagger}\right]=\delta_{\beta}^{\alpha} \delta_{b}^{a}, \quad\left[q^{\alpha a}, q^{\beta b}\right]=\left[q_{\alpha a}^{\dagger}, q_{\beta b}^{\dagger}\right]=0 .
$$

It is useful to introduce the round bracket notation in order to distinguish the states in the spin-flavour Fock space from the states defined in (2.12). A spin-flavour state with

\footnotetext{
${ }^{5}$ For a more precise description of this statement see Appendix E.
} 
baryon quantum numbers is then given by

$$
\left.\mid \mathcal{B})=\mathcal{B}^{\alpha_{1} a_{1} \ldots \alpha_{N_{c} a_{N_{c}}}} q_{\alpha_{1} a_{1}}^{\dagger} \ldots q_{\alpha_{N_{c}} a_{N_{c}}}^{\dagger} \mid 0\right),
$$

|0) being the vacuum state of the spin-flavour Fock space. Upon the replacement ${ }^{6}$

$$
\left.b_{0, \alpha a A}^{\dagger} \rightarrow q_{\alpha a}^{\dagger}, \quad b_{0, \alpha a A} \rightarrow q^{\alpha a}, \quad|\cdot\rangle \rightarrow \mid \cdot\right), \quad \hat{\mathcal{O}}^{(r)} \rightarrow \mathcal{O}^{(r)},
$$

the expansion for the matrix elements in (2.16) can be given in an analogous form:

$$
\left\langle\mathcal{B}^{\prime}\left|\mathcal{T} \hat{\mathcal{O}}_{1}\left(x_{1}\right) \ldots \hat{\mathcal{O}}_{n}\left(x_{n}\right)\right| \mathcal{B}\right\rangle=\sum_{l} \sum_{r=1}^{N_{c}} c_{r}^{l}\left(x_{1}, \ldots, x_{n}\right)\left(\mathcal{B}^{\prime}\left|\mathcal{O}_{l}^{\left(r_{l}\right)}\right| \mathcal{B}\right),
$$

with the normal-ordered operators

$$
\mathcal{O}^{(r)}=q_{\beta_{1} b_{1}}^{\dagger} \ldots q_{\beta_{r} b_{r}}^{\dagger} \mathcal{O}_{\left(\alpha_{1} a_{1}\right) \ldots\left(\alpha_{r} a_{r}\right)}^{\left(\beta_{1} b_{1}\right) \ldots\left(\beta_{r} b_{r}\right)} q^{\alpha_{1} a_{1}} \ldots q^{\alpha_{r} a_{r}} .
$$

Here, $\mathcal{O}_{\left(\alpha_{1} a_{1}\right) \ldots\left(\alpha_{r} a_{r}\right)}^{\left(\beta_{1} b_{1}\right) \ldots\left(\beta_{r} b_{r}\right)}$ are the matrix elements of the new introduced operators $\mathcal{O}^{(r)}$, which act in the spin-flavour space only. Using this result, the evaluation of matrix elements of arbitrary QCD operators simplifies to the calculation of matrix elements of effective spin-flavour operators - a procedure familiar from the non-relativistic quark models. The price for this simplification is the introduction of unknown functions $c_{r}^{l}$.

We proceed with the discussion of the expansion in terms of the effective operators. Given a QCD Heisenberg operator with certain spin-flavour transformation properties, one has to determine a minimal set of linear independent effective $r$-body operators. Having stated the transformation properties of the unknown coefficients $c_{r}^{l}$, this amounts to the determination of all possible spin-flavour tensors $\mathcal{O}_{\left(\alpha_{1} a_{1}\right) \ldots\left(\alpha_{r} a_{r}\right)}^{\left(\beta_{1} b_{1}\right) \ldots\left(\beta_{r} b_{r}\right)}$ in $(2.22)$ with appropriate spin-flavour transformation properties. These tensors must be traceless and symmetric. A non-traceless tensor would correspond to a lower-body operator. An antisymmetric (or partially symmetric) tensor would vanish if evaluated on the totally symmetric baryonic states in the spin-flavour Fock space.

Another possibility to specify the effective operators is to express all $r$-body operators as a product of $r$ one-body operators, whose matrix elements can be evaluated more easily than the matrix elements of (2.22):

$$
\mathcal{O}^{(r)}=\mathcal{O}_{1}^{(1)} \mathcal{O}_{2}^{(1)} \ldots \mathcal{O}_{r}^{(1)}
$$

The one-body operators are most easily classified according to the different representation of the spin and flavour symmetry groups. The operator basis is furnished by the following set of one-body operators:

$$
\begin{aligned}
\mathbb{1} & =q^{\dagger}(\mathbf{1} \otimes \mathbf{1}) q, & J^{i} & =q^{\dagger}\left(\frac{\sigma^{(i)}}{2} \otimes \mathbf{1}\right) q, \\
T^{a} & =q^{\dagger}\left(\mathbf{1} \otimes \frac{\lambda^{(a)}}{2}\right) q, & G^{i a} & =q^{\dagger}\left(\frac{\sigma^{(i)}}{2} \otimes \frac{\lambda^{(a)}}{2}\right) q,
\end{aligned}
$$

\footnotetext{
${ }^{6}$ It is also possible to discuss the anti-quarks in a similar manner. This makes an introduction of spin-flavour creation and annihilation operators for the anti-quarks necessary and can be used to study the physics of exotic baryons in the large- $N_{c}$ limit (see e. g. [53]).
} 
1 stands here for the corresponding identity matrices in spin and flavour spaces. An $r$-body operator can be written in this basis as a sum over all independent polynomials in the one-body operators:

$$
\mathcal{O}^{(r)}=\sum_{m, n} c_{n m}(J)^{m}(T)^{n}(G)^{r-m-n}, \quad m, n \in \mathbb{N},
$$

with some coefficients $c_{n m}$, which needn't be specified further here. For such products of $r$ one-body operators it is not guaranteed any more, that they correspond to $r$-body normal ordered operators in (2.22), which transform under some irreducible representation of the spin-flavour symmetry group. Viz., such products can contain lower-body contributions or can build tensors, which are reducible ${ }^{7}$.

Upon normal ordering and using the bosonic commutator relation for the spin-flavour quark operators, this is easily demonstrated by means of two simple examples:

$$
\begin{aligned}
4 J^{i} T^{a} & =q_{\beta_{1} b_{1}}^{\dagger}\left(\sigma^{(i)}\right)_{\alpha_{1}}^{\beta_{1}} q^{\alpha_{1} b_{1}} q_{\beta_{2} b_{2}}^{\dagger}\left(\lambda^{(a)}\right)_{a_{2}}^{b_{2}} q^{\beta_{2} a_{2}} \\
& =4 G^{i a}+q_{\beta_{1} b_{1}}^{\dagger} q_{\beta_{2} b_{2}}^{\dagger} \mathcal{O}_{\left(\alpha_{1} a_{1}\right)\left(\alpha_{2} a_{2}\right)}^{\left(\beta_{1} b_{1}\right)\left(\beta_{2} b_{2}\right)} q^{\alpha_{1} a_{1}} q^{\alpha_{2} a_{2}}
\end{aligned}
$$

with

$$
\mathcal{O}_{\left(\alpha_{1} a_{1}\right)\left(\alpha_{2} a_{2}\right)}^{\left(\beta_{1} b_{1}\right)\left(\beta_{2} b_{2}\right)}=\left(\sigma^{(i)}\right)_{\alpha_{1}}^{\beta_{1}} \delta_{a_{1}}^{b_{1}} \delta_{\alpha_{2}}^{\beta_{2}}\left(\lambda^{(a)}\right)_{a_{2}}^{b_{2}}
$$

and

$$
\begin{aligned}
4 T^{a} T^{b} & =q_{\beta_{1} b_{1}}^{\dagger}\left(\lambda^{(a)}\right)_{a_{1}}^{b_{1}} q^{\beta_{1} a_{1}} q_{\beta_{2} b_{2}}^{\dagger}\left(\lambda^{(b)}\right)_{a_{2}}^{b_{2}} q^{\beta_{2} a_{2}} \\
& =4 q^{\dagger}\left(\lambda^{(a)} \lambda^{(b)}\right) q+q_{\beta_{1} b_{1}}^{\dagger} q_{\beta_{2} b_{2}}^{\dagger} \mathcal{O}_{\left(\alpha_{1} a_{1}\right)\left(\alpha_{2} a_{2}\right)}^{\left(\beta_{1} b_{1}\right)\left(\beta_{2} b_{2}\right)} q^{\alpha_{1} a_{1}} q^{\alpha_{2} a_{2}},
\end{aligned}
$$

with

$$
\mathcal{O}_{\left(\alpha_{1} a_{1}\right)\left(\alpha_{2} a_{2}\right)}^{\left(\beta_{1} b_{1}\right)\left(\beta_{2} b_{2}\right)}=\delta_{\alpha_{1}}^{\beta_{1}}\left(\lambda^{(a)}\right)_{a_{1}}^{b_{1}} \delta_{\alpha_{2}}^{\beta_{2}}\left(\lambda^{(b)}\right)_{a_{2}}^{b_{2}}
$$

Both of these products of two one-body operators, $J^{i} T^{a}$ and $T^{a} T^{b}$, contain upon normal ordering non-traceless components, which lead to the pure one-body operators. The products of two lambda-matrices in $T^{a} T^{b}$ are further reducible.

Furthermore, certain combinations of products of one-body operators do not represent a totally symmetric operators upon normal-ordering and vanish therefore, when acting on the baryon states. Since only genuine symmetric $r$-body operators are needed in the expansion in (2.21), such redundant or vanishing products/combinations must be eliminated. This is done by means of a set of operator identities, which are the main topic of the next section.

An one-body operator can be inserted on any of the $N_{c}$ quark lines in the baryon - the matrix elements of an one-body operator are of the order $N_{c}$. Generalizing this result,

\footnotetext{
${ }^{7}$ These two cases correspond to tensors $\mathcal{O}_{\left(\alpha_{1} a_{1}\right) \ldots\left(\alpha_{r} a_{r}\right)}^{\left(\beta_{1} b_{1}\right) \ldots\left(\beta_{r} b_{r}\right)}$ in $(2.25)$ which are not trace-less or are reducible, respectively.
} 
the matrix elements of an $r$-body scale like $N_{c}^{r}{ }^{8}$ Therefore, despite the explicit factor $1 / N_{c}^{r-1}$ in (2.21), all possible $r$-body operators $\mathcal{O}^{(r)}$ appear to be of equal importance. Since the evaluation of an infinite number of operators pose an intractable problem, the benefit of such an expansion is questionable at the first sight.

Building baryons out of $N_{c}$ quark leads inevitably to spin-flavour multiplets, which do not exist in the physical case of $N_{c}=3$ (see e.g.[56, 21]). The ambiguity in the selection of a tower of baryon states, which is bound to the physical states for $N_{c}=3$, is the way out of this problem. At this point it is not necessary to specify such a tower explicitly. It is only sufficient to notice, that the possible large- $N_{c}$ baryon states can be restricted to those states, for which the matrix elements of $J$ are $\mathcal{O}\left(N_{c}^{0}\right)$ for all values of $N_{c}$ and which collapse to the physical baryons for $N_{c}=3$. On the other hand, the insertions of the other one-body operators, $T$ and $G$, add coherently. Matrix elements of these operators confirm the naive expectation for a one-body operator and scale like $N_{c}$. I.e.

$$
\left(\mathcal{B}^{\prime}\left|J^{i}\right| \mathcal{B}\right) \sim N_{c}^{0}, \quad\left(\mathcal{B}^{\prime}\left|T^{a}\right| \mathcal{B}\right) \sim N_{c}, \quad\left(\mathcal{B}^{\prime}\left|G^{i a}\right| \mathcal{B}\right) \sim N_{c}
$$

In this case, each occurrence of $J$ is suppressed by a factor of $1 / N_{c}$. This allows to terminate the expansion in (2.21) at a finite number of terms for each order in $1 / N_{c}$.

\subsubsection{Operator identities}

The classification of the minimal set of independent operators with given transformation properties in the expansion (2.21) is a group-theoretical problem. As already mentioned, doing the expansion in terms of normal ordered operators asks for finding all tensor structures $\mathcal{O}_{\left(\alpha_{1} a_{1}\right) \ldots\left(\alpha_{r} a_{r}\right)}^{\left(\beta_{1} b_{1}\right)}$ in $(2.22)$ that transform under some irreducible representation of $S U(2) \otimes S U\left(N_{F}\right)$. On the other hand, using products of one-body operators in (2.23) leads to a more straightforward evaluation of the matrix elements but requires elimination of irrelevant or vanishing parts in such products or combination of them. This is done by a set of operator identities derived in [22]. Main results of the cited work are summarised in this section, whereas the main steps which has to be done in the course of derivation of these identities are sketched in Appendix G. The expansion in (2.21) is valid for an arbitrary number of flavours. For the sake of straight notation, the rest of this work will be limited to the case of only interest, $N_{F}=3$.

The only relevant operator identities are those which relate products of two one-body operators to one-body and zero-body operators ${ }^{9}$. Identities for all products of $r$ onebody operators with $r>2$ are obtained by recursively applying the two-body identities on all pairs of one-body operators.

Furthermore, for the physical case $N_{c}=3$ one can stop the expansion at products which contain three one-body operators at most. Normal ordering of such products with $r>3$

\footnotetext{
${ }^{8}$ There are $N_{c}$ possibilities to choose the first line, $N_{c}-1$ possibilities for the second line a.s.o. So the overall factor is $N_{c}\left(N_{c}-1\right) \ldots\left(N_{c}-r-1\right) \sim N_{c}^{r}$. This result can also be easily read off $(2.25)$.

${ }^{9}$ There is also a trivial identity relating the one-body operator $\mathbb{1}$ to the zero-body operator: $\mathbb{1}=N_{c}$.
} 
one-body operators results in three- and/or lower-body operators and in operators with $r>3$ quark-creation and annihilation operators on the left and on the right hand sides. The later vanish if acting on the baryon states in (2.19) for $N_{c}=3$.

The operators introduced in (2.24) obey the Lie-algebra of the group $S U(6)^{10}$ :

$$
\begin{aligned}
& {\left[J^{i}, J^{j}\right]=i \varepsilon^{i j k} J^{k}, \quad\left[T^{a}, T^{b}\right]=i f^{a b c} T^{c}, \quad\left[J^{i}, T^{a}\right]=0,} \\
& {\left[J^{i}, G^{j a}\right]=i \varepsilon^{i j k} G^{k a}, \quad\left[T^{a}, G^{i b}\right]=i f^{a b c} G^{i c}} \\
& {\left[G^{i a}, G^{j b}\right]=\frac{i}{4} \delta^{i j} f^{a b c} T^{c}+\frac{i}{6} \delta^{a b} \varepsilon^{i j k} J^{k}+\frac{i}{2} \varepsilon^{i j k} d^{a b c} G^{k c} .}
\end{aligned}
$$

A product of two one-body operators can always be written as a sum of an antisymmetric and a symmetric product using a commutator or an anticommutator, respectively. Since the commutator of two one-body operators can always be eliminated (reduced to a onebody operator) by using the commutator relations in (2.31), only symmetric products need to be taken into account.

Operators, similar to the vectors in the carrier space where they are acting, can be lumped in sets, that transform under different irreducible representations of the symmetry group. Usually, a product of two one-body operators contains several components that transform according to different irreducible representations of $S U(2) \otimes S U(3)$. Not all of these components in the product are independent among each other. Furthermore, many of them can be reduced to one-body operators or vanish when acting on the totally symmetric spin-flavour baryon states.

In total, there are 22 different components in the symmetric products of $J, T$ and $G$ operators. Careful analysis of these components, sketched in Appendix G, leads to 12 relations between them. These operator identities, given in Table G.1, reduce the number of independent components to ten. Obviously, the decision about what subset of these components to promote to be "relevant" is not unique. The operator identities allow to select a certain subset of relevant operators in such a way that the following mnemonic operation reduction rule for all the redundant parts can be formulated:

- All operator products in which two flavour indices are contracted using $\delta^{a b}, f^{a b c}$ or $d^{a b c}$ or two spin indices on $G^{\prime}$ 's are contracted using $\delta^{i j}$ or $\epsilon^{i j k}$ can be eliminated with the help of the operator identities.

- All operator products in which two flavour indices are contracted using symmetric or antisymmetric combinations of two different $d$ and/or $f$ symbols $\left(d^{a c g} d^{b c h} \pm d^{a c g} d^{a c h}\right.$, etc.) can be rewritten with the help of several identities of $d$ and $f$ in (G.24) and can be eliminated afterwards with the help of the operator identities. The only exception to this rule is the antisymmetric combination $f^{a c g} d^{b c h}-f^{b c g} d^{a c h}$.

\footnotetext{
${ }^{10}$ In $[43,48]$ the representations of the symmetry group of the meson-baryon interaction in large- $N_{c}$ limit are shown to follow from contraction of $S U\left(2 N_{F}\right)$. It is argued, that for case of $N_{F}=3$ the symmetry group $S U(6)$, taken for granted in the nonrelativistic quark models, is just a consequence of the physics in large- $N_{c}$ limit. The generators of the spin-flavour group and its subgroups, $G, J, T$, are introduced and the matrix elements of QCD operators are written as a function of matrix elements of these generators. This leads to an expression similar to (2.21) [22]. In the approach advocated in [63], these operators arise simply as a tool for classifying the spin- and flavour properties of quark-gluon diagrams. Regardless of the chosen approach, the operators obey the $\mathfrak{s u}(6)$ Lie-algebra.
} 
To see the operator reduction rule at work, we show explicitly how all the redundant combinations in the products of two one-body operators can be rewritten. It holds:

$$
\begin{aligned}
\left\{T^{a}, T^{a}\right\} & =\frac{1}{6}\left(N_{c}+6\right) \mathbb{1}+\left\{J^{k}, J^{k}\right\}, \\
d^{a b c}\left\{T^{a}, T^{b}\right\} & =-\frac{1}{3}\left(N_{c}+3\right) T^{c}, \\
\left\{T^{a}, G^{i a}\right\} & =\frac{2}{3}\left(N_{c}+3\right) J^{i}, \\
d^{a b c}\left\{T^{a}, G^{i b}\right\} & =\frac{1}{3}\left(N_{c}+3\right) G^{i c}+\frac{1}{6}\left\{J^{i}, T^{c}\right\}, \\
f^{a b c}\left\{T^{a}, G^{i b}\right\} & =\varepsilon^{i j k}\left\{J^{j}, G^{k c}\right\}, \\
\left\{G^{i a}, G^{j a}\right\} & =\frac{1}{8} \delta^{i j}\left(\left(N_{c}+6\right) \mathbb{1}-2\left\{J^{k}, J^{k}\right\}\right)+\frac{1}{3}\left\{J^{i}, J^{j}\right\}, \\
d^{a b c}\left\{G^{i a}, G^{j b}\right\} & =\frac{1}{3} \delta^{i j}\left(\frac{4}{3}\left(N_{c}+3\right) T^{c}-\frac{3}{2}\left\{J^{k}, G^{k c}\right\}\right) \\
& +\frac{1}{6}\left(\left\{J^{i}, G^{j c}\right\}+\left\{J^{j}, G^{i c}\right\}\right), \\
\left\{G^{k a}, G^{k b}\right\} & =\frac{1}{24} \delta^{a b}\left(\left(N_{c}+6\right) \mathbb{1}-2\left\{J^{k}, J^{k}\right\}\right) \\
& +\frac{1}{2} d^{a b c}\left(\left(N_{c}+3\right) T^{c}-2\left\{J^{k}, G^{k c}\right\}\right)+\frac{1}{4}\left\{T^{a}, T^{b}\right\}, \\
\varepsilon^{i j k}\left\{G^{j a}, G^{k b}\right\} & =\frac{1}{2} f^{a b c}\left(-\left(N_{c}+3\right) G^{i c}+\frac{1}{6}\left\{J^{i}, T^{c}\right\}\right) \\
& +\frac{1}{2}\left(f^{a c g} d^{b c h}-f^{b c g} d^{a c h}\right)\left\{T^{g}, G^{i h}\right\} .
\end{aligned}
$$

Note the application of the 2nd part of the reduction rule in the last line. The decomposition of all symmetric products of two one-body operators into different irreducible parts and elimination of the redundant parts by means of the operator identities is given at the end of Appendix G. The expessions in (2.32) follow directly from that analysis and prove, together with the $S U(3)$ relations for the $f$-and $d$-symbols

$$
\begin{aligned}
d_{a g c} d_{b h c}+d_{a h c} d_{b g c} & =\frac{1}{3}\left(\delta_{a b} \delta_{g h}+\delta_{a g} \delta_{b h}+\delta_{a h} \delta_{b g}\right)-d_{a b c} d_{g h c} \\
d_{a g c} d_{b h c}-d_{a h c} d_{b g c} & =f_{a b c} f_{g h c}-\frac{2}{3}\left(\delta_{a g} \delta_{b h}-\delta_{a h} \delta_{b g}\right) \\
f_{a g c} f_{b h c}+f_{a h c} f_{b g c} & =\delta_{a b} \delta_{g h}-\delta_{a g} \delta_{b h}-\delta_{a h} \delta_{b g}+3 d_{a b c} d_{g h c} \\
f_{a g c} f_{b h c}-f_{a h c} f_{b g c} & =f_{a b c} f_{g h c} \\
f_{a g c} d_{b h c}+f_{a h c} d_{b g c} & =f_{a b c} d_{g h c}
\end{aligned}
$$

the two reduction rules stated above. 


\subsection{Chiral Lagrangian in the large- $N_{c}$ limit}

As it was shown in the previous section of this chapter, a systematic $1 / N_{c}$-expansion of the physical properties of baryons, which are closely connected to various matrix elements of quark operators, can be carried out with the help of effective spin-flavour quark operators. On the other hand, an effective Lagrangian, which exploits the symmetries of the underlying theory, provides a convenient method to determine the low-energy behaviour of such matrix elements. The connection between both, the low-energy and the $1 / N_{c}$ expansions, is established by evaluating the matrix elements of quark operators under consideration in both approaches and by matching the corresponding spin and flavour structures.

The low energy expansion is parameterised by coupling constants in the chiral Lagrangian introduced in Section 1.4. The $1 / N_{c}$-expansion is accompanied by the unknown " $N_{c^{-}}$ couplings" $c_{r}^{l}$ in (2.21). Given the number of $N_{c}$-couplings smaller then the number of chiral couplings, such a matching furnish a set of large- $N_{c}$ constraints for the chiral parameters.

We summarise the steps. Given a quark operator, the expansion in small momenta and small quark masses of the baryonic matrix elements is evaluated by taking appropriate functional derivatives of the transition amplitude $\mathcal{F}\left(\vec{p}^{\prime}, \vec{p} ; v, a, s, p\right)$ in (1.21) with respect to external fields. The large- $N_{c}$ expansion is obtained by evaluating matrix elements of spin-flavour quark operators as in (2.23). In the course of the later expansion, the operator reduction rule is used to determine the relevant products of the effective quark operators $^{11}$. These operators act in the space of baryon spin-flavour states, $\left.\mid \mathcal{B}\right)$. The spin-flavour states of interest in this work are members of the flavour $S U(3)$ octet and decuplet multiplets and are denoted in the following by

$$
\mid a, \chi), \quad \text { and } \quad|i j k, \chi\rangle \text {. }
$$

Here, the spin-polarization index takes the values $\chi=1,2$ for the spin- $1 / 2$ baryons of the octet and $\chi=1, \cdots, 4$ for the spin-3/2 baryons of the decuplet and the flavour indices are $a=1, \cdots, 8$ and $i, j, k=1,2,3$, respectively. The construction of these spin-flavour states and the evaluation of one- and two-body operators, taken between these states, are discussed in Appendices D and $\mathrm{E}$ in detail. The action of the one-body quark operators on these states can be given in a transparent form (see also [64]):

$$
\begin{aligned}
\left.J_{i} \mid a, \chi\right) & \left.=\frac{1}{2} \sigma_{\bar{\chi} \chi}^{(i)} \mid a, \bar{\chi}\right), \\
\left.T^{a} \mid b, \chi\right) & \left.=i f_{b c a} \mid c, \chi\right), \\
\left.G_{i}^{a} \mid b, \chi\right) & \left.\left.=\sigma_{\bar{\chi} \chi}^{(i)}\left(\frac{1}{2} d_{b c a}+\frac{i}{3} f_{b c a}\right) \mid c, \bar{\chi}\right)+\frac{1}{2 \sqrt{2}} S_{\bar{\chi} \chi}^{(i)} \Lambda_{a b}^{k l m} \mid k l m, \bar{\chi}\right), \\
\left.J_{i} \mid k l m, \chi\right) & \left.=\frac{3}{2}\left(\vec{S} \sigma_{i} \vec{S}^{\dagger}\right)_{\bar{\chi} \chi} \mid k l m, \bar{\chi}\right),
\end{aligned}
$$

\footnotetext{
${ }^{11}$ See also Table G.2 and the discussion in Appendix G.
} 


\begin{tabular}{|c|c|c|c|}
\hline T-odd & T-even & P-odd & P-even \\
\hline $\begin{array}{c}J_{i} G_{i}^{a} \\
f_{a b c}\end{array}$ & $\begin{array}{c}T^{a} \\
\delta_{a b} d_{a b c}\end{array}$ & $f_{a b c}$ & $\begin{array}{ccc}J_{i} & G_{i}^{a} & T_{a} \\
\delta_{a b} & d_{a b c}\end{array}$ \\
\hline
\end{tabular}

Table 2.1.: Transformation properties of the objects used in the effective expansion in (2.21) under parity and time reversal.

$$
\begin{aligned}
\left.T^{a} \mid k l m, \chi\right) & \left.=\frac{3}{2} \Lambda_{k l m}^{a, n o p} \mid n o p, \chi\right) \\
\left.G_{i}^{a} \mid k l m, \chi\right) & \left.\left.=\frac{3}{4}\left(\vec{S} \sigma_{i} \vec{S}^{\dagger}\right)_{\bar{\chi} \chi} \Lambda_{k l m}^{a, n o p} \mid n o p, \bar{\chi}\right)+\frac{1}{2 \sqrt{2}}\left(S_{i}^{\dagger}\right)_{\bar{\chi} \chi} \Lambda_{k l m}^{a b} \mid b, \bar{\chi}\right),
\end{aligned}
$$

with the spin-transition matrices $\sigma, S$ (see Appendix F) and the flavour transition tensors $\Lambda$ introduced in (1.51).

Furthermore, the expansion in terms of the effective operators has to obey the proper behavior under the discrete transformations of the Heisenberg quark operator under consideration. This can be fulfilled with the help of Table 2.1 summarising the transformation properties of the objects used in the $1 / N_{c}$-expansion.

In the following three sections the matching of both expansions is carried out for the baryon masses, for the matrix elements of the axial vector and for a product of two axial vector quark currents. The analysis is limited to the tree level results in the low-energy expansion obtained in Section 1.4.

\subsubsection{Baryon masses}

There is an extensive treatment of the baryon masses in the literature done in the framework of the $1 / N_{c}$-expansion $[22,50,7,70]$. In this section a connection between the mass pattern, as implied by this expansion, and the perturbative chiral expansion for baryon masses, as implied by the symmetry breaking part of the chiral Lagrangian, is established.

Baryon masses are obtained by evaluating the matrix elements of the QCD-Hamiltonian. In the limit of exact $S U(3)$ symmetry this QCD-operator transforms as a singlet in the spin and in the flavour spaces. Therefore, the expansion in the basis of the effective operators is given to the leading and sub-leading order by

$$
M_{\mathcal{B}}=\left(\mathcal{B}\left|c_{1}^{(0,1)} \mathbb{1}+c_{2}^{(0,1)} J^{2}\right| \mathcal{B}\right)
$$

Matrix elements of the first operator scale like $\sim N_{c}$. This reflects the fact, that the baryons consist of $N_{c}$ quarks. The parameter $c_{1}^{(0,1)}$ can be regarded as the mass unit per color degree of freedom. In the limit $N_{c} \rightarrow \infty$ the baryon states are degenerate. The first correction to this degeneracy is provided by the matrix elements of the $J^{2}$ operator which 


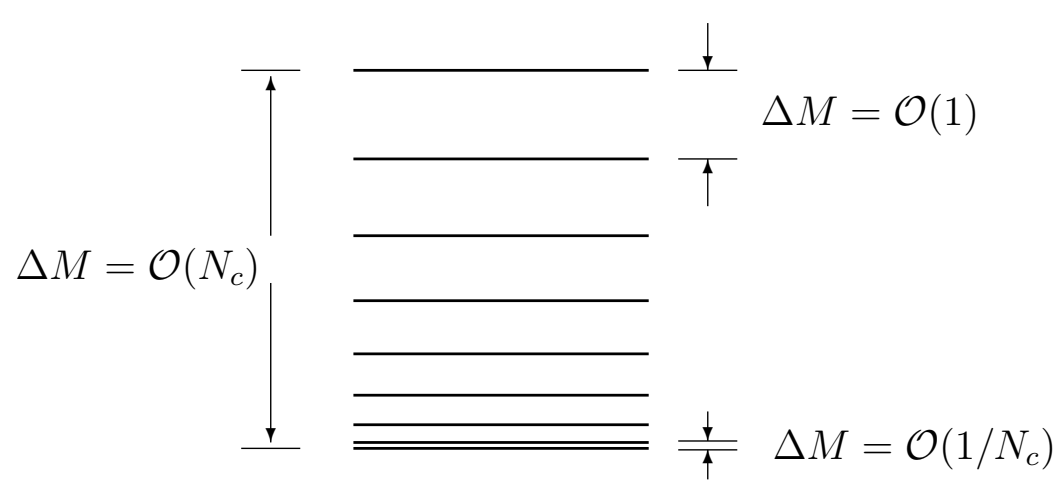

Figure 2.7.: The baryon mass spectrum as implied by the expansion in (2.36) and the mass splitting in (2.37) for different regions. The top and the bottom of the tower denote baryons with $j=N / 2$ and $j=1 / 2$, respectively.

is responsible for the mass splitting between multiplets of different spin. It holds:

$$
M_{J}-M_{J^{\prime}} \sim \frac{1}{N_{c}}\left(j(j+1)-j^{\prime}\left(j^{\prime}+1\right)\right) .
$$

$N_{c}$ spin-1/2 quarks can be coupled to a baryon with a total spin ranging from $j=1 / 2$ to $j=N_{c} / 2$. Concerning the spin-structure only, the baryon tower can be represented as in Figure 2.7. The expansion in terms of effective operators in (2.21) is only meaningful for the baryons at the bottom of this tower, where the matrix elements of the spin-operator $J$ are suppressed relative to other operators. This was already discussed an the end of the Section 2.3.1.

For the physical baryons it is known, that the $S U(3)$ flavour symmetry is not exact. It is possible to study the mass spectrum by combining the effects of the flavour symmetry breaking with the $1 / N_{c}$ expansion. Mass relations which follow from such a combined expansion go beyond the relations, which can be obtained from the study of the breaking of the flavour $S U(3)$-symmetry or the combined spin-flavour $S U(6)$-symmetry only.

For three quark flavours, the breaking of the $S U(3)$ symmetry is taken into account in QCD-Lagrangian by the quark mass matrix with $m_{s} \neq m^{12}$ :

$$
\begin{aligned}
\operatorname{diag}\left(m, m, m_{s}\right) & =\frac{1}{3}\left(2 m+m_{s}\right) \mathbb{1}_{3 \times 3}+\frac{1}{\sqrt{3}}\left(m-m_{s}\right) \lambda^{(8)} \\
& \equiv \frac{1}{3} M \mathbb{1}_{3 \times 3}+\frac{1}{\sqrt{3}} \varepsilon \lambda^{(8)}
\end{aligned}
$$

The parameters $M$ and $\varepsilon$ introduced in the last line control the strength of the $S U(3)$ flavour symmetry and its breaking. It holds:

$$
M=2 m+m_{s}, \quad \varepsilon=m-m_{s}, \quad \text { or } \quad m=(M+\varepsilon) / 3, \quad m_{s}=(M-2 \varepsilon) / 3 .
$$

The quark mass matrix transforms as $(\mathbf{1} \oplus \mathbf{8})$ in the flavour space. The deviation from the exact $S U(3)$ symmetry transforms as the eighth component of the flavour octet.

\footnotetext{
${ }^{12}$ Exact isospin-symmetry $m=m_{u}=m_{d}$ is assumed here.
} 
Therefore, the combined expansion in $1 / N_{c}$ and $\varepsilon$ for the baryon masses is obtained by adding all possible $(\mathbf{0}, \mathbf{8})$ spin-flavour operators to (2.36) and setting the open flavour index to 8 . To the first order in the flavour symmetry breaking, the $\left\{J^{i}, G^{i 8}\right\}$ operator provides the first $1 / N_{c}$-correction to the leadig operator $T^{8}$ :

$$
M_{\mathcal{B}}=\left(\mathcal{B}\left|c_{1}^{(0,1)} \mathbb{1}+c_{2}^{(0,1)} J^{2}+\varepsilon c_{1}^{(0,8)} T^{8}+\varepsilon c_{2}^{(0,8)}\left\{J^{i}, G^{i 8}\right\}\right| \mathcal{B}\right) .
$$

At second order in $\varepsilon$, the perturbation transforms as $(\mathbf{1} \oplus \mathbf{8}) \otimes(\mathbf{1} \oplus \mathbf{8})$ in the flavour space. From the decomposition of $\mathbf{8} \otimes \mathbf{8}$ it follows, that additional to the singlet and octet operators in $(2.40)$ one needs to consider $(\mathbf{1 0}+\overline{\mathbf{1 0}})$ - and $\mathbf{2 7}$-operators with the open flavour indices set to 8 . The first one vanishes and there is only one additional operator $\left\{T^{8}, T^{8}\right\}^{13}$ :

$$
M_{B}=\left(\mathcal{B}\left|c_{1}^{(0,1)} \mathbb{1}+c_{2}^{(0,1)} J^{2}+\varepsilon c_{1}^{(0,8)} T^{8}+\varepsilon c_{2}^{(0,8)}\left\{J^{i}, G^{i 8}\right\}+\varepsilon^{2} c_{2}^{(0,27)}\left\{T^{8}, T^{8}\right\}\right| \mathcal{B}\right) .
$$

Here, the subtraction terms in the $\left\{T^{8}, T^{8}\right\}$-operator projecting onto the pure 27 -component, were not written out since they can be completely absorbed in the terms already included in $(2.41)$.

For the sake of completeness, the expansion of the baryon masses, according to (2.41), is given here for the octet and for the decuplet baryons:

$$
\begin{aligned}
& M_{N}=c_{1}^{(0,1)}+\frac{3}{4} c_{2}^{(0,1)}+\frac{\sqrt{3}}{2} \varepsilon c_{1}^{(0,8)}+\frac{\sqrt{3}}{4} \varepsilon c_{2}^{(0,8)}+\frac{3}{2} \varepsilon^{2} c_{2}^{(0,27)}, \\
& M_{\Lambda}=c_{1}^{(0,1)}+\frac{3}{4} c_{2}^{(0,1)}-\frac{\sqrt{3}}{2} \varepsilon c_{2}^{(0,8)}, \\
& M_{\Sigma}=c_{1}^{(0,1)}+\frac{3}{4} c_{2}^{(0,1)}+\frac{\sqrt{3}}{2} \varepsilon c_{2}^{(0,8)}, \\
& M_{\Xi}=c_{1}^{(0,1)}+\frac{3}{4} c_{2}^{(0,1)}-\frac{\sqrt{3}}{2} \varepsilon c_{1}^{(0,8)}-\frac{3 \sqrt{3}}{4} \varepsilon c_{2}^{(0,8)}+\frac{3}{2} \varepsilon^{2} c_{2}^{(0,27)}, \\
& M_{\Delta}=c_{1}^{(0,1)}+\frac{15}{4} c_{2}^{(0,1)}+\frac{\sqrt{3}}{2} \varepsilon c_{1}^{(0,8)}+\frac{5 \sqrt{3}}{4} \varepsilon c_{2}^{(0,8)}+\frac{3}{2} \varepsilon^{2} c_{2}^{(0,27)}, \\
& M_{\Sigma}=c_{1}^{(0,1)}+\frac{15}{4} c_{2}^{(0,1)}, \\
& M_{\Xi}=c_{1}^{(0,1)}+\frac{15}{4} c_{2}^{(0,1)}-\frac{\sqrt{3}}{2} \varepsilon c_{1}^{(0,8)}-\frac{5 \sqrt{3}}{4} \varepsilon c_{2}^{(0,8)}+\frac{3}{2} \varepsilon^{2} c_{2}^{(0,27)}, \\
& M_{\Omega}=c_{1}^{(0,1)}+\frac{15}{4} c_{2}^{(0,1)}-\sqrt{3} \varepsilon c_{1}^{(0,8)}-\frac{5 \sqrt{3}}{2} \varepsilon c_{2}^{(0,8)}+6 \varepsilon^{2} c_{2}^{(0,27)} .
\end{aligned}
$$

We discuss the relation between various baryon masses, as implied by (2.42), order by order in the $\varepsilon$-expansion. The meaning of $c_{1}^{(0,1)}$ and $c_{2}^{(0,1)}$ was already discussed above. To the first order in the $\varepsilon$-expansion, eight baryon masses are given as a function of four

\footnotetext{
${ }^{13}$ The operator $d^{88 c} T^{c}$ doesn't need to be considered explicitly because of $d^{88 c} T^{c}=d^{888} T^{8}=-\frac{1}{\sqrt{3}} T^{8}$, which is already included in $(2.40)$.
} 
unknown coefficients. This results in four linearly independent mass relations:

$$
\begin{aligned}
\frac{3}{4} M_{\Lambda}+\frac{1}{4} M_{\Sigma}-\frac{1}{2}\left(M_{N}+M_{\Xi}\right) & =0 \\
M_{\Sigma_{[10]}}-M_{\Delta} & =M_{\Xi_{[10]}}-M_{\Sigma_{[10]}}, \\
M_{\Omega}-M_{\Xi_{[10]}} & =M_{\Xi_{[10]}}-M_{\Sigma_{[10]}}, \\
M_{\Sigma_{[10]}}-M_{\Sigma} & =M_{\Xi_{[10]}}-M_{\Xi} .
\end{aligned}
$$

The first line is the Gell-Mann-Okubo relation, the following two are the decuplet equal spacing rules. These relations also follow from the pure $S U(3)$-analysis. The last identity relates masses of different flavour multiplets to each other and is a consequence of the first $1 / N_{c}$-correction that was taken into account in (2.40) by the $\left\{J^{i}, G^{i 8}\right\}$-operator.

Up to the second order in $\varepsilon$ the baryon masses are parameterised by 5 unknown coefficients in (2.41). Relations between the masses resulting from this parameterisation are:

$$
\begin{gathered}
\frac{3}{4} M_{\Lambda}+\frac{1}{4} M_{\Sigma}-\frac{1}{2}\left(M_{N}+M_{\Xi}\right)=\frac{1}{4}\left(\left(M_{\Sigma_{10}}-M_{\Delta}\right)-\left(M_{\Omega}-M_{\Xi_{10}}\right)\right), \\
\left(M_{\Omega}-M_{\Xi_{10}}\right)-2\left(M_{\Xi_{[10]}}-M_{\Sigma_{[10]}}\right)+\left(M_{\Sigma_{10}}-M_{\Delta}\right)=0, \\
M_{\Sigma_{[10]}}-M_{\Sigma}=M_{\Xi_{[10]}}-M_{\Xi} .
\end{gathered}
$$

The right hand side of the first identity, a linear combination of two decuplet equal spacing rules, provides a correction to the Gell-Mann-Okubo relation. The second identity is also a linear combination of decuplet equal spacing rules. The last identity was already obtained in (2.43).

Mass relations in (2.43) and (2.44) were obtained by considering the $S U(3)$ flavour symmetry breaking perturbatively. Similar analysis can also be performed without assuming the $S U(3)$ symmetry at all and treating only the breaking of the isospin symmetry perturbatively. The flavour symmetry group to use in this case is $S U(2) \times U(1)$. Mass relations obtained by studying this symmetry group are valid to all orders in the $S U(3)$ symmetry breaking parameter $\varepsilon$. A detailed analysis of both scenarios shows that the perturbative treatment of the symmetry breaking produce a better accuracy in mass relations compared to the case of the completely broken $S U(3)$ flavour symmetry [50]. Furthermore, flavour expansion supplemented by the $1 / N_{c}$ expansion, as it was done in this section, leads to accurate relations, which are not available if one does these expansions separately.

Guided by the success of the combined expansion in $\varepsilon$ and in $1 / N_{c}$, the pattern of baryon masses, as provided by such an expansion, is imposed in the following on the corresponding parameters in the chiral Lagrangian. This is done by a straightforward matching of the results for the baryon masses obtained in Section 1.4.3 to the corresponding expressions in (2.42).

In the effective Lagrangian the role of the first contribution to the baryon masses from $c_{1}^{(0,1)}$ and its spin-dependent $c_{2}^{(0,1)}$-correction are taken over by the different baryon masses 
in the chiral limit $-\stackrel{\circ}{M}_{[8]}$ for the spin-1/2 baryons in the octet and $\stackrel{\circ}{M}_{\text {[10] }}$ for the spin-3/2 baryons in the decuplet. Furthermore, the explicit breaking of chiral symmetry and the breaking of the $S U(3)$ flavour symmetry in the chiral Lagrangian is taken into account via the explicit occurrence of the quark mass matrix in $\chi_{0}=2 B_{0} \operatorname{diag}\left(m, m, m_{s}\right)$.

To the leading order in $\varepsilon$, the expansion in (2.40) corresponds to the mass shifts linear in $\chi_{0}$. Matching of both results yields for the chiral couplings:

$$
\begin{gathered}
b_{0}=-\frac{1}{4 B_{0} M}\left(c_{1}^{(0,1)}+\frac{3}{4} c_{2}^{(0,1)}\right)+\frac{\sqrt{3}}{8 B_{0}} c_{2}^{(0,8)}, \\
b_{D}=-\frac{3 \sqrt{3}}{16 B_{0}} c_{2}^{(0,8)}, \quad b_{F}=-\frac{\sqrt{3}}{8 B_{0}}\left(c_{1}^{(0,8)}+c_{2}^{(0,8)}\right), \\
d_{0}=-\frac{1}{4 B_{0} M}\left(c_{1}^{(0,1)}+\frac{15}{4} c_{2}^{(0,1)}\right)+\frac{\sqrt{3}}{16 B_{0}}\left(2 c_{1}^{(0,8)}+5 c_{2}^{(0,8)}\right), \\
d_{D}=-\frac{3 \sqrt{3}}{16 B_{0}}\left(2 c_{1}^{(0,8)}+5 c_{2}^{(0,8)}\right) .
\end{gathered}
$$

In the chiral expansion of the baryon masses the parameters $b_{0}$ and $d_{0}$ renormalise the values of the masses in the chiral limit, $\stackrel{\circ}{M}_{8}$ and $\stackrel{\circ}{M}_{10}$ respectively ${ }^{14}$. The dependence of these chiral parameters on $c_{1}^{(0,1)}$ and $c_{2}^{(0,1)}$ in (2.4.1) restates this fact.

As the result of the expressions in (2.4.1), the $Q^{2}$ chiral symmetry breaking coupling constants are subject to several large- $N_{c}$ constraints. To the leading order in the $1 / N_{c^{-}}$ expansion it holds:

$$
b_{D}=0, \quad d_{0}-\frac{1}{3} d_{D}=b_{0}, \quad d_{D}=3 b_{F} .
$$

The sub-leading corrections to these results, taken into account by the $J^{2}$ and $\left\{J^{i}, G^{i 8}\right\}$ operators in (2.41), lead to

$$
d_{D}=3\left(b_{D}+b_{F}\right) .
$$

The $Q^{4}$-contributions to the baryon masses provide the $\varepsilon^{2}$-pattern of the explicit symmetry breaking. Matching of the corresponding mass shifts to the results in (2.42) leads to

$$
\begin{gathered}
c_{0}=\frac{3}{8 B_{0}^{2}} c_{2}^{(0,27)}, \quad c_{1}=\frac{3}{4 B_{0}^{2}} c_{2}^{(0,27)}, \quad c_{2}=-\frac{9}{8 B_{0}^{2}} c_{2}^{(0,27)}, \quad c_{3}=0 \\
c_{4}=-\frac{3 \sqrt{3}}{16 M B_{0}^{2}} c_{2}^{(0,8)}+\frac{3}{4 B_{0}^{2}} c_{2}^{(0,27)}, \quad c_{5}=-\frac{\sqrt{3}}{8 M B_{0}^{2}}\left(c_{1}^{(0,8)}+c_{2}^{(0,8)}\right), \\
c_{6}=-\frac{1}{4 M^{2} B_{0}^{2}}\left(c_{1}^{(0,1)}+\frac{3}{4} c_{2}^{(0,1)}\right)+\frac{\sqrt{3}}{8 M B_{0}^{2}} c_{2}^{(0,8)}-\frac{3}{8 B_{0}^{2}} c_{2}^{(0,27)},
\end{gathered}
$$

\footnotetext{
${ }^{14}$ Such renormalization is inherent to the chiral perturbation theory and is not limited to the masses.
} 
and

$$
\begin{gathered}
e_{0}=0, \quad e_{1}=\frac{9}{4 B_{0}^{2}} c_{2}^{(0,27)}, \quad e_{2}=-\frac{27}{8 B_{0}^{2}} c_{2}^{(0,27)}, \\
e_{3}=-\frac{3 \sqrt{3}}{16 M B_{0}^{2}}\left(2 c_{1}^{(0,8)}+5 c_{2}^{(0,8)}\right)+\frac{9}{4 B_{0}^{2}} c_{2}^{(0,27)}, \\
e_{4}=-\frac{1}{4 M^{2} B_{0}^{2}}\left(c_{1}^{(0,1)}+\frac{15}{4} c_{2}^{(0,1)}\right)+\frac{\sqrt{3}}{16 M B_{0}^{2}}\left(2 c_{1}^{(0,8)}+5 c_{2}^{(0,8)}\right)-\frac{3}{8 B_{0}^{2}} c_{2}^{(0,27)}
\end{gathered}
$$

The renormalization of the $Q^{2}$ terms by the $Q^{4}$ terms was already noted in (1.73). By noting the dependence of the $Q^{4}$ chiral couplings on $\varepsilon^{0}$ and $\varepsilon$ large- $N_{c}$ parameters, we recover this behaviour in (2.48) and (2.49).

Several relations between the $Q^{4}$ chiral couplings can be established. To the leading order in the $1 / N_{c}$-expansion they read

$$
\begin{gathered}
c_{1}=2 c_{0}, \quad c_{2}=-3 c_{0}, \quad c_{3}=0, \quad c_{4}=2 c_{0}, \\
e_{0}=0, \quad e_{1}=6 c_{0}, \quad e_{2}=-9 c_{0}, \quad e_{3}=3\left(2 c_{0}+c_{5}\right), \quad e_{4}=c_{6}-c_{5} .
\end{gathered}
$$

The $1 / N_{c}$ corrected results are

$$
\begin{gathered}
c_{1}=2 c_{0}, \quad c_{2}=-3 c_{0}, \quad c_{3}=0, \\
e_{0}=0, \quad e_{1}=6 c_{0}, \quad e_{2}=-9 c_{0}, \quad e_{3}=3\left(c_{4}+c_{5}\right) .
\end{gathered}
$$

The discussion of the baryon masses is closed by an additional remark. Mass relations which follow from (2.42) are independent of the actual values of the quark masses. Therefore, the validness of the combined expansion in $\varepsilon$ and in $1 / N_{c}$ can be tested order by order using the results of chiral extrapolations and of lattice-QCD calculations, carried out at different quark masses. Such an analysis was recently carried out in [54].

\subsubsection{Axial-vector-couplings}

The leading order structure of the axial-vector quark currents, evaluated between baryon states, in the low-energy expansion was discussed in Section 1.4.1. To investigate the corresponding large- $N_{c}$ operator expansion, we limit the discussion to the space components. The axial-vector current $\mathcal{A}^{i a}(x),(i=1,2,3)$, transforms as $(\mathbf{1}, \mathbf{8})$ under the space and flavour rotations. Doing the expansion in (2.21), vector indices can be distributed between the unknown coefficients $c_{r}^{l}$ and the effective operators. Leading terms in the momentum expansion of the matrix elements of an axial vector in (1.50) don't show any momentum dependence. Therefore, the unknown coefficients in the effective expansion are taken to be independent of the baryon momenta, $c_{r}^{l}(\bar{p}, p)=c_{r}^{l}$, and the vector properties are carried by the effective operators only. 
Using Table G.2, all the relevant $(\mathbf{1}, \mathbf{8})$-operators are readily found. The leading terms in the $1 / N_{c^{-}}$expansion for the Fourier-transformed of (1.48) are:

$$
\left\langle\bar{p}, \bar{\chi}\left|\mathcal{A}^{i a}(0)\right| \vec{p}, \chi\right\rangle=\left(\bar{\chi}\left|c_{1}^{(1,8)} G^{i a}+c_{2}^{(1,8)}\left\{J^{i}, T^{a}\right\}\right| \chi\right) .
$$

In contrast to the operators in the above expansion, the third possible $(\mathbf{1}, \mathbf{8})$-operator $\varepsilon^{i j k}\left\{J^{j}, G^{k a}\right\}$ is even under time reversal. Since the axial vector current is $\mathcal{T}$-odd, this operator is not allowed in the $1 / N_{c}$ expansion in (2.52). Further possible operators are combinations of the operators given above and additional powers of $J^{2}$. These operators are suppressed by at least a factor of $1 / N_{c}^{2}$ relative to the operators included above.

Additional subtle point is the discussion of the $N_{c}$ scaling of the matrix elements, as was mentioned at the end of the Section 2.3.1. The one-body operator $G^{i a}$ doesn't scale like $N_{c}$ everywhere in the weight diagram for baryons consisting of $N_{c}$ quarks. Therefore, the two-body operator $\left\{J^{i}, T^{a}\right\}$, though explicitly suppressed in (2.52) by the factor $1 / N_{c}$, needs to be retained for a consistent expansion.

To show the interplay between the $1 / N_{c^{-}}$and the chiral expansions, the evaluation of the corresponding matrix elements in both expansions is given in an explicit form in the following. The large- $N_{c}$ operator

$$
\mathcal{O}^{i a}=c_{1}^{(1,8)} G^{i a}+c_{2}^{(1,8)}\left\{J^{i}, T^{a}\right\}
$$

is evaluated between the baryon spin-flavour states $\mid a, \chi)$ and $\mid i j k, \chi)$. It holds:

$$
\begin{aligned}
\left(c, \bar{\chi}\left|\mathcal{O}^{i a}\right| b, \chi\right) & =\sigma_{\bar{\chi} \chi}^{(i)}\left(c_{1}^{(1,8)} \frac{1}{2} d^{a b c}+\left(\frac{1}{3} c_{1}^{(1,8)}+c_{2}^{(1,8)}\right) i f^{a b c}\right), \\
\left(n o p, \bar{\chi}\left|\mathcal{O}^{i a}\right| b, \chi\right) & =S_{\bar{\chi} \chi}^{(i)} c_{1}^{(1,8)} \frac{1}{2 \sqrt{2}} \Lambda_{a b}^{n o p}, \\
\left(n o p, \bar{\chi}\left|\mathcal{O}^{i a}\right| k l m, \chi\right) & =\left(\vec{S} \sigma^{(i)} \vec{S}^{\dagger}\right)_{\bar{\chi} \chi} \frac{3}{4}\left(c_{1}^{(1,8)}+6 c_{2}^{(1,8)}\right) \Lambda_{k l m}^{a, x y z} \delta_{x y z}^{n o p} .
\end{aligned}
$$

Matching of (2.54) with (1.50) leads directly to

$$
D=\frac{1}{2} c_{1}^{(1,8)}, \quad F=\frac{1}{3} c_{1}^{(1,8)}+c_{2}^{(1,8)}, \quad C=c_{1}^{(1,8)}, \quad H=\frac{3}{2}\left(c_{1}^{(1,8)}+6 c_{2}^{(1,8)}\right) .
$$

This result, first obtained in [22], provides two relations between the meson-baryon coupling constants:

$$
C=2 D, \quad H=-3 D+9 F .
$$

At leading order, the four coupling constants are parameterised by the matrix elements of $G^{i a}$ only. The relations for the constants read in this case:

$$
F=2 / 3 D, \quad C=2 D, \quad H=3 D .
$$

This result is also obtained in the quark models with the assumed $S U(6)$ spin-flavour symmetry group, where the generator $G^{i a}$ is evaluated between the $S U(6)$ symmetric baryon wave functions. The difference between (2.56) and (2.57) is due to the sub-leading effect taken into account by the $\left\{J^{i}, T^{a}\right\}$-operator in the $1 / N_{c^{-}}$-expansion in (2.53). 


\subsubsection{Meson baryon four-point interaction}

The subject of interest in this section is the $Q^{2}$ four-point interaction stated in Section 1.4.2. The connection of these interaction terms to the quark-gluon physics at large $N_{c}$ is established via the matrix elements of quark operators as in (1.59).

The relevant effective quark operators are constrained by the transformation properties of (1.59). In the following we limit the discussion to the space components of the axial vector quark currents only. The decomposition of the product of two axial vector currents, where each of them transforms as $(\mathbf{1}, \mathbf{8})$ under spatial and flavour rotations, is

$$
(1,8) \otimes(1,8)=(0,8 \otimes 8) \oplus(1,8 \otimes 8) \oplus(2,8 \otimes 8),
$$

where the product of two flavour octets decomposes as follows:

$$
8 \otimes 8=1+8_{\mathrm{S}}+8_{\mathrm{A}}+10+\overline{10}+27 .
$$

Doing the expansion (2.21) for the product of two axial vector currents, it is not quite obvious how to distribute the vector indices between the effective operators and the momentum dependent coefficients $c_{r}^{l}$. Since we are mainly interested in the structure of the $Q^{2}$ four-point interaction terms, the kinematic properties of those terms can serve as a guide in this case. Examination of the momentum dependence in the explicit results for the chiral expansions in $(1.64$ - 1.66) furnish the ansatz for the momentum dependence of the coefficients in the corresponding $1 / N_{c}$ expansion. The "remnant" vector indices are carried by the effective operators. The flavour structures of the operators are determined according to (2.58) and are mapped to the flavour structures in the chiral interaction.

Having stated the spin and flavour structures, the relevant operators are readily determined with the help of Table G.2. Putting all together, the Fourier-transformed of the product of two axial quark currents, as defined in (1.63), is

$$
\begin{aligned}
& \left\langle\mathcal{B}^{\prime}\left(p^{\prime}\right)\left|C_{i j}^{a b}(\bar{p}-p)\right| \mathcal{B}(\vec{p})\right\rangle \\
& \quad=-\delta_{i j}\left(\mathcal{B}^{\prime}\left|c_{1}^{(0,1)} \delta^{a b} \mathbb{1}+c_{1}^{(0,8)} d^{a b c} T^{c}+\frac{1}{4} c_{2}^{(0,27)} \mathcal{P}^{(0,27)}\left\{T^{a}, T^{b}\right\}\right| \mathcal{B}\right) \\
& \quad+\frac{(p+\bar{p})_{i}(p+\bar{p})_{j}}{4 M}\left(\mathcal{B}^{\prime}\left|\tilde{c}_{0}^{(0,1)} \delta^{a b} \mathbb{1}+\tilde{c}_{1}^{(0,8)} d^{a b c} T^{c}+\frac{1}{4} \tilde{c}_{2}^{(0,27)} \mathcal{P}^{(0,27)}\left\{T^{a}, T^{b}\right\}\right| \mathcal{B}\right) \\
& \quad+i \frac{\left(\varepsilon_{i k l}(p+\bar{p})_{j}+\varepsilon_{j k l}(p+\bar{p})_{i}\right)(p-\bar{p})_{k}}{4 M}\left(\mathcal{B}^{\prime}\left|c_{1}^{(1,8)} d^{a b c} G^{l c}+\frac{1}{4} c_{2}^{(1,27)} \mathcal{P}^{(1,27)}\left\{T^{a}, G^{l b}\right\}\right| \mathcal{B}\right) \\
& +\varepsilon_{i j k}\left(\mathcal{B}^{\prime}\left|\tilde{c}_{1}^{(1,8)} f^{a b c} G^{k c}+\frac{1}{4} \tilde{c}_{2}^{(1, \overline{10}+10)} \mathcal{P}^{(1, \overline{10}+10)} f^{a c g} d^{b c h}\left\{T^{g}, G^{k h}\right\}\right| \mathcal{B}\right) \\
& +\left(\mathcal{B}^{\prime}\left|\frac{1}{4} c_{2}^{(2,27)} \mathcal{P}^{(2,27)}\left\{G^{i a}, G^{j b}\right\}\right| \mathcal{B}\right) \\
& +\ldots
\end{aligned}
$$

To stress it again, the ansatz for the momentum dependence of the unknown coefficients $c_{r}^{l}$, which was written out explicitly in the above expression, is not the most general one. It is rather motivated by the structures of the $Q^{2}$ contact terms in the chiral 
Lagrangian. The omitted terms, denoted by ... in (2.60), reflect the structure of terms in the chiral Lagrangian which are either of higher order (say, have more derivatives on baryon and/or meson fields) or correspond to the pole-terms in (1.61). To the order in the chiral expansion we are interested in, it is legitimate and fully sufficient to perform the matching of the chiral and $1 / N_{c^{-}}$expansions on the basis of $(2.60)$.

The projectors $\mathcal{P}^{l}$ embody in a compact form subtraction terms as in (B.21) and (B.22) and guarantee the operators, which they are acting on, to transform under some definite irreducible spin-flavour representation $l$. In general, those subtraction terms are either already included in the effective expansion or are of sub-leading order. E.g., the 27-plet component in $\left\{T^{a}, T^{b}\right\}$ is obtained by means of the following subtractions:

$$
\begin{aligned}
\mathcal{P}^{(0,27)}\left\{T^{a}, T^{b}\right\} & \equiv\left\{T^{a}, T^{b}\right\}-\frac{1}{8} \delta^{a b}\left(\frac{1}{6}\left(N_{c}+6\right) \mathbb{1}+\left\{J^{k}, J^{k}\right\}\right) \\
& -\frac{3}{5} d^{a b c}\left(-\frac{1}{3}\left(N_{c}+3\right) T^{c}+2\left\{J^{k}, G^{k c}\right\}\right) .
\end{aligned}
$$

In this way, the projection onto definite multiplets guarantees the leading order behavior of the operators included in the $1 / N_{c^{-}}$expansion. Expressions similar to $(2.61)$ hold also for other operators and their corresponding subtraction terms. Details can be found in Appendix G.

Evaluation of the ansatz in (2.60) for the octet and decuplet baryon states and matching to the corresponding results in $(1.64-1.66)$ leads to the correlations between the chiral and the $1 / N_{c}$-couplings. For the chiral coupling constants in the vertices containing octet fields only it holds:

$$
\begin{gathered}
g_{0}^{(S)}=6 c_{1}^{(0,1)}-\frac{11}{20} c_{2}^{(0,27)}, \quad g_{1}^{(S)}=-2 c_{2}^{(0,27)}, \\
g_{D}^{(S)}=\frac{6}{5} c_{2}^{(0,27)}, \quad g_{F}^{(S)}=2 c_{1}^{(0,8)}, \\
g_{0}^{(V)}=6 \tilde{c}_{1}^{(0,1)}-\frac{11}{20} \tilde{c}_{2}^{(0,27)}, \quad g_{1}^{(V)}=-2 \tilde{c}_{2}^{(0,27)}, \\
g_{D}^{(V)}=\frac{6}{5} \tilde{c}_{2}^{(0,27)}, \quad g_{F}^{(V)}=2 \tilde{c}_{1}^{(0,8)}, \\
g_{0}^{(V)}=-\frac{4}{3} c_{1}^{(1,8)}-\frac{11}{30} c_{2}^{(1,27)}, \quad g_{1}^{(V)}=-\frac{4}{3} c_{2}^{(1,27)}, \\
g_{D}^{(V)}=2 c_{1}^{(1,8)}+\frac{4}{5} c_{2}^{(1,27)}, \quad g_{F}^{(V)}=\frac{4}{3} c_{1}^{(1,8)}, \\
g_{1}^{(T)}=-\tilde{c}_{2}^{(1, \overline{0} 0+10)}, \quad g_{D}^{(T)}=-\tilde{c}_{1}^{(1,8)}, \\
g_{F}^{(T)}=-\frac{2}{3} \tilde{c}_{1}^{(1,8)}+\frac{1}{3} \tilde{c}_{2}^{(1, \overline{1} 0+10)} .
\end{gathered}
$$


For the vertices containing the decuplet fields only, the correlations are given by:

$$
\begin{gathered}
\bar{h}_{1}^{(S)}=6 c_{1}^{(0,1)}-2 c_{1}^{(0,8)}+\frac{3}{10} c_{2}^{(0,27)}, \quad \bar{h}_{2}^{(S)}=3 c_{1}^{(0,8)}+\frac{9}{5} c_{2}^{(0,27)}, \quad \bar{h}_{3}^{(S)}=-3 c_{2}^{(0,27)} \\
h_{1}^{(V)}=6 \tilde{c}_{1}^{(0,1)}-2 \tilde{c}_{1}^{(0,8)}+\frac{3}{10} \tilde{c}_{2}^{(0,27)}, \quad h_{2}^{(V)}=3 \tilde{c}_{1}^{(0,8)}+\frac{9}{5} \tilde{c}_{2}^{(0,27)}, \quad h_{3}^{(V)}=-3 \tilde{c}_{2}^{(0,27)}, \\
h_{1}^{(V)}=-2 c_{1}^{(1,8)}+\frac{3}{10} c_{2}^{(1,27)}, \quad h_{2}^{(V)}=3 c_{1}^{(1,8)}+\frac{9}{5} c_{2}^{(1,27)}, \quad h_{3}^{(V)}=-3 c_{2}^{(1,27)} \\
h_{1}^{(T)}=-\frac{3}{2} \tilde{c}_{1}^{(1,8)}, \\
h_{2}^{(S)}=\frac{3}{20} c_{2}^{(2,27)}, \quad h_{4}^{(S)}=\frac{9}{10} c_{2}^{(2,27)}, \quad h_{6}^{(S)}=-\frac{3}{2} c_{2}^{(2,27)}
\end{gathered}
$$

with $^{15}$

$$
\bar{h}_{1}^{(S)}=h_{1}^{(S)}+\frac{1}{3} h_{2}^{(S)}, \quad \bar{h}_{2}^{(S)}=h_{3}^{(S)}+\frac{1}{3} h_{4}^{(S)}, \quad \bar{h}_{3}^{(S)}=h_{5}^{(S)}+\frac{1}{3} h_{6}^{(S)} .
$$

Finally, analogous matching for the vertices with both, the octet and the decuplet baryons, results in

$$
\begin{gathered}
f_{2}^{(A)}=-4 \tilde{c}_{1}^{(1,8)}-2 \tilde{c}_{2}^{(1, \overline{10}+10)}, \quad f_{4}^{(A)}=6 \tilde{c}_{2}^{(1, \overline{10}+10)} \\
f_{1}^{(A)}=\frac{1}{5} c_{2}^{(2,27)}, \quad f_{3}^{(A)}=c_{2}^{(2,27)} .
\end{gathered}
$$

Similar to the results in the previous two sections, the correlations in (2.4.3-2.65) provide several relations between the different chiral couplings. In the following, these relations are grouped according to the spin-structure of the effective operators, which the chiral coupling constants are matched to.

Spin-0:

$$
\begin{gathered}
g_{D}^{(S)}=-\frac{3}{5} g_{1}^{(S)}, \quad \bar{h}_{1}^{(S)}=g_{0}^{(S)}-\frac{17}{40} g_{1}^{(S)}-g_{F}^{(S)}, \\
\bar{h}_{2}^{(S)}=\frac{3}{2}\left(g_{D}^{(S)}+g_{F}^{(S)}\right), \quad \bar{h}_{3}^{(S)}=\frac{3}{2} g_{1}^{(S)}
\end{gathered}
$$

\footnotetext{
${ }^{15}$ Upon the tensor reduction, the $Q^{2}$ interaction terms in (1.54) accomponied by the coupling constants $h_{2}^{(S)}, h_{4}^{(S)}$ and $h_{6}^{(S)}$ contribute to the spin-0 and spin-2 structures in the matrix elements of the product of two axial quark currents. The "renormalization" of the pure spin-0 couplings $h_{1}^{(S)}, h_{3}^{(S)}$ and $h_{5}^{(S)}$ by them is expressed by the abbreviations in (2.64).
} 


$$
\begin{gathered}
g_{D}^{(V)}=-\frac{3}{5} g_{1}^{(V)}, \quad h_{1}^{(V)}=g_{0}^{(V)}-\frac{17}{40} g_{1}^{(V)}-g_{F}^{(V)}, \\
h_{3}^{(V)}=\frac{3}{2}\left(g_{D}^{(V)}+g_{F}^{(V)}\right), \quad h_{5}^{(V)}=\frac{3}{2} g_{1}^{(V)} .
\end{gathered}
$$

Spin-1:

$$
\begin{gathered}
g_{D}^{(V)}=-\frac{3}{16}\left(8 g_{0}^{(V)}+g_{1}^{(V)}\right), \quad g_{F}^{(V)}=-g_{0}^{(V)}+\frac{11}{40} g_{1}^{(V)}, \\
h_{1}^{(V)}=\frac{3}{4}\left(2 g_{0}^{(V)}-\frac{17}{20} g_{1}^{(V)}\right), \quad h_{2}^{(V)}=-\frac{9}{4}\left(g_{0}^{(V)}+\frac{13}{40} g_{1}^{(V)}\right), \\
h_{3}^{(V)}=\frac{9}{4} g_{1}^{(V)}, \\
g_{F}^{(T)}=\frac{1}{3}\left(2 g_{D}^{(T)}-g_{1}^{(T)}\right), \quad h_{1}^{(T)}=\frac{3}{2} g_{D}^{(T)}, \\
f_{2}^{(P V)}=2\left(g_{1}^{(T)}+2 g_{D}^{(T)}\right), \quad f_{4}^{(P V)}=-6 g_{1}^{(T)} .
\end{gathered}
$$

Spin-2:

$$
\begin{array}{cl}
h_{4}^{(S)}=12 h_{2}^{(S)}, & h_{6}^{(S)}=-20 h_{2}^{(S)}, \\
f_{1}^{(P V)}=-\frac{8}{3} h_{2}^{(S)}, & f_{3}^{(P V)}=-\frac{40}{3} h_{2}^{(S)} .
\end{array}
$$

The five groups in (2.66-2.70) correspond to the five lines in the ansatz for the expansion of the product of two axial-vector quark currents in terms of the effective quark operators in $(2.60)$. 
The evaluation of the properly subtracted effective operators guarantees the leading order behavior in the $1 / N_{c}$ expansion, involves, however, a lot of tedious algebra. The subtraction terms can be omitted, if they can be absorbed by another operators which are explicitly included in the expansion. On the other hand, inclusion of several unsubtracted operators can lead to a redundant and/or a suppressed contribution, if evaluated only in a subset of the baryon states.

We discuss this by means of the following ansatz ${ }^{16}$ :

$$
\begin{aligned}
i \int d^{4} x e^{-i(p \prime-p) x}\left\langle\mathcal{B}^{\prime}(\bar{p})\left|\mathcal{T} \mathcal{A}^{i a}(x) \mathcal{A}^{j b}(0)\right| \mathcal{B}(p)\right\rangle \\
\quad=-\delta^{i j}\left(\mathcal{B}^{\prime}\left|g_{1}\left(\frac{1}{3} \delta^{a b} \mathbb{1}+d^{a b c} T^{c}\right)+\frac{1}{2} g_{2}\left\{T^{a}, T^{b}\right\}\right| \mathcal{B}\right) \\
\quad+\frac{(p+\bar{p})^{i}(p+\bar{p})^{j}}{4 M}\left(\mathcal{B}^{\prime}\left|g_{3}\left(\frac{1}{3} \delta^{a b} \mathbb{1}+d^{a b c} T^{c}\right)+\frac{1}{2} g_{4}\left\{T^{a}, T^{b}\right\}\right| \mathcal{B}\right) \\
\quad+\varepsilon^{i j k}\left(\mathcal{B}^{\prime}\left|g_{5} f^{a b c} G^{k c}\right| \mathcal{B}\right)+\left(\mathcal{B}^{\prime}\left|\frac{1}{2} g_{6}\left\{G^{i a}, G^{j b}\right\}+\frac{1}{2} g_{7}\left\{G^{j a}, G^{i b}\right\}\right| \mathcal{B}\right) \\
\quad+\ldots
\end{aligned}
$$

In labeling the unknown $1 / N_{c}$-coefficients in the above ansatz we are in no accordance with the notation $c_{r}^{l}$ in (2.16), since we don't insist on the definit multiplet structures in the products of the effective operators.

The particular combination of operators associated with $g_{1}$ and $g_{3}$ follows since the onebody operator contribution results from quark-gluon diagrams where the flavor matrices $\lambda^{a}$ and $\lambda^{b}$ sit on a single quark line leading to the unique flavor structure $\lambda^{a} \lambda^{b}=$ $\frac{2}{3} \delta^{a b}+\left(d_{a b c}+i f_{a b c}\right) \lambda^{c}$. By disregarding the suppressed case, where this line is closed (quark loop), this implies that the symmetric part of the leading order one-body operator contributes in the combination $\frac{1}{3} \delta^{a b} \mathbb{1}+d^{a b c} T^{c}$ always.

We note that according to the reduction rule the operator

$$
\epsilon^{i j k}\left(f^{a c g} d^{b c h}-f^{b c g} d^{a c h}\right)\left\{T^{g}, G^{k h}\right\}
$$

is not redundant and should be considered in (2.71) in addition. However, due to the last equation in (2.32) and

$$
\left\{G^{i a}, G^{j b}\right\}-\left\{G^{j a}, G^{i b}\right\}=\varepsilon_{i j k} \varepsilon_{k l m}\left\{G^{l a}, G^{m b}\right\},
$$

it follows that the two operators $\left\{G^{i a}, G^{j b}\right\}$ and $\left\{G^{j a}, G^{i b}\right\}$ in (2.71) may be linearly combined to the structure (2.72) modulo operators that are already considered or that are subleading. Thus, by allowing a partialy suppressed effect it is legitimate to consider the combination (2.73) instead of (2.72).

Evaluation of the ansatz (2.71) and the explicit matching to the contributions of the

\footnotetext{
${ }^{16}$ The kinematic dependence for the ansatz is fixed by the chiral expansion and is the same as in the previous ansatz. In the following we disregard the first spin-1 component of the ansatz in (2.60).
} 
two-body terms to the matrix elements of the correlator $C_{i j}^{(a b)}$ in $(1.64-1.66)$ leads to

$$
\begin{gathered}
g_{0}^{(S)}=2 g_{1}-g_{2}-\frac{7}{12} g_{+}, \quad g_{1}^{(S)}=-2 g_{2}+\frac{1}{6} g_{+}, \\
g_{D}^{(S)}=3 g_{2}+\frac{1}{4} g_{+}, \quad g_{F}^{(S)}=2 g_{1}-\frac{2}{3} g_{+}, \\
g_{0}^{(V)}=2 g_{3}-g_{4}, \quad g_{1}^{(V)}=-2 g_{4}, \\
g_{D}^{(V)}=3 g_{4}, \quad g_{F}^{(V)}=2 g_{3}, \\
g_{1}^{(T)}=-\frac{1}{2} g_{-}, \quad g_{D}^{(T)}=-g_{5}+\frac{1}{2} g_{-}, \quad g_{F}^{(T)}=-\frac{2}{3} g_{5}+\frac{5}{12} g_{-},
\end{gathered}
$$

and

$$
\begin{gathered}
h_{1}^{(S)}=0, \quad h_{2}^{(S)}=0, \quad h_{3}^{(S)}=3 g_{1}+\frac{9}{2} g_{2}-\frac{9}{8} g_{+}, \\
h_{4}^{(S)}=\frac{3}{2} g_{+}, \quad h_{5}^{(S)}=-3 g_{2}+\frac{3}{4} g_{+}, \quad h_{6}^{(S)}=-\frac{3}{2} g_{+}, \\
h_{1}^{(V)}=0, \quad h_{2}^{(V)}=3 g_{3}+\frac{9}{2} g_{4}, \quad h_{3}^{(V)}=-3 g_{4}, \\
h_{1}^{(T)}=-\frac{3}{2} g_{5}+\frac{3}{8} g_{-},
\end{gathered}
$$

and

$$
\begin{gathered}
f_{1}^{(A)}=0, \quad f_{2}^{(A)}=-4 g_{5}+g_{-}, \\
f_{3}^{(A)}=g_{+}, \quad f_{4}^{(A)}=3 g_{-},
\end{gathered}
$$

with

$$
g_{ \pm}=\frac{1}{2}\left(g_{6} \pm g_{7}\right)
$$

The correlations in $(2.74-2.76)$ result in 17 sum-rules:

$$
\begin{gathered}
g_{F}^{(S)}=g_{0}^{(S)}-\frac{1}{2} g_{1}^{(S)}, \quad h_{1}^{(S)}=0, \quad h_{2}^{(S)}=0, \\
h_{3}^{(S)}=\frac{3}{2} g_{0}^{(S)}-\frac{9}{4} g_{1}^{(S)}+\frac{1}{2} g_{D}^{(S)}, \quad h_{4}^{(S)}=3\left(g_{D}^{(S)}+\frac{3}{2} g_{1}^{(S)}\right), \\
h_{5}^{(S)}=g_{D}^{(S)}+3 g_{1}^{(S)}, \quad h_{6}^{(S)}=-3\left(g_{D}^{(S)}+\frac{3}{2} g_{1}^{(S)}\right), \\
g_{D}^{(V)}=-\frac{3}{2} g_{1}^{(V)}, \quad g_{F}^{(V)}=g_{0}^{(V)}-\frac{1}{2} g_{1}^{(V)}, \\
h_{1}^{(V)}=0, \quad h_{2}^{(V)}=\frac{3}{2} g_{0}^{(V)}-3 g_{1}^{(V)}, \quad h_{3}^{(V)}=\frac{3}{2} g_{1}^{(V)},
\end{gathered}
$$




$$
\begin{gathered}
g_{F}^{(T)}=-\frac{1}{6} g_{1}^{(T)}+\frac{2}{3} g_{D}^{(T)}, \quad h_{1}^{(T)}=\frac{3}{4}\left(g_{1}^{(T)}+2 g_{D}^{(T)}\right), \\
f_{1}^{(A)}=0, \quad f_{2}^{(A)}=2\left(g_{1}^{(T)}+2 g_{D}^{(T)}\right), \quad f_{4}^{(A)}=-6 g_{1}^{(T)} .
\end{gathered}
$$

In total, 25 chiral couplings are correlated to each other by 7 unknown "large- $N_{c}$ couplings" either in (2.60) or in (2.71). The differences between the both ansatzes are of sub-leading order. 


\section{Chapter 3.}

\section{Quark-mass dependence of the baryon masses}

In this chapter we extend the results of [79] to the order $Q^{4}$. We discuss the implication of the chiral loop expansion, as described below, for the extrapolation of the baryon masses and compare with the results of recent lattice simulations.

The results from a strict chiral expansion of the baryon masses, are unable to describe the almost linear quark mass dependence as shown on the lattice (see e.g. [30]). In the baryon sector, a polynomial fit may be used instead of the predictions of $\chi \mathrm{PT}$. Extrapolated to the physical point, the deviation from the experimental values is within few percent $[1]^{1}$. Though such a linear extrapolation may seem to be satisfactory for lattice practitioners, an understanding of the failure and a correct description within the framework of an effective field theory in the baryon sector are desirable and still lacking.

This problem is tightly related to the problem of very poor convergence properties in the baryon sector with tree flavours. The poor convergence manifests itself by very large dimensional regularised loop-modifications of the leading order results. This was realised soon after the formulation of $\chi \mathrm{PT}$ for baryons in [39] and is observed in various calculations of different observables (see e.g. [25] for a brief overview) ${ }^{2}$. The situation was improved by introducing a finite cutoff into the loop integrals $[25,26,11,8]$. Years later alternative regularisation schemes were proposed [28, 6, 40, 41, 32, 79]. These schemes supplement the dimensional regularisation and the $\overline{\mathrm{MS}}$-renormalisation by additional subtractions in the integrals and restore thereby the correct power-counting in the relativistic formulation of $\chi \mathrm{PT}$ in the baryon sector.

The convergence pattern is strongly improved within the $\chi$ MS-scheme as it was demonstrated for the case of baryon masses in [79]. In this scheme the convergence pattern can be controlled by the infrared scale. The introduction of an additional scale doesn't have any impact on the physical quantities since it can be completely absorbed by counter terms. However, the presence of such a scale reorganises the perturbative expansion and can lead to a better convergence pattern by choosing an appropriate value for it. Besides the correct power-counting, this scheme takes loop contributions, as implied by the relativistic theory, completely into account - the leading order terms in loop contributions

\footnotetext{
${ }^{1}$ Such small deviations can still be assigned to the finite lattice effects.

${ }^{2}$ Originally, the observation in [39], that a strict application of the $\overline{\mathrm{MS}}$-scheme in the baryon sector contradicts to the power-counting rules, an indispensable tool in an effective expansion, led to the nonrelativistic formulation of $\chi \mathrm{PT}$ in [51].
} 
always obey the expectation of the power-counting rules and the strict chiral expansion is superseded by the chiral loop expansion. Such a loop expansion corresponds to a partial summation of higher order terms and improves the convergence properties further.

\subsection{Baryon masses in the chiral loop-expansion}

To the order $Q^{4}$ the self energy receives contributions from tree-level diagrams and oneloop diagrams:

$$
\Sigma_{B}\left(M_{B}\right)=\Sigma_{B}^{\text {tree-level }}+\Sigma_{B}^{\text {loop }}, \quad B \in[8],[10],
$$

where the index $B$ stands for the members of the flavor $S U(3)$ octet and decuplet. In this work we apply the $\chi \mathrm{MS}$ scheme developed in [79]. The physical mass of the baryon $M_{B}$ is determined by the condition

$$
M_{B}-\Sigma_{B}\left(M_{B}\right)=\left\{\begin{array}{lll}
\stackrel{\circ}{M}_{[8]} & \text { for } & B \in[8] \\
\stackrel{\circ}{M}_{[10]} & \text { for } & B \in[10]
\end{array} .\right.
$$

where $\stackrel{\circ}{M}_{[8]}$ and $\stackrel{\circ}{M}_{[8]}$ are the corresponding baryon masses in the chiral limit.

The $Q^{3}$ one-loop corrections ${ }^{3}$ to the baryon masses from the leading order interaction Lagrangian in (1.45) were calculated in [79]. Here we state the results only and refer to the original work for the details of the calculation ${ }^{4}$.

The $Q^{4}$ tadpole-contributions to the octet and decuplet baryon masses are calculated in Appendix A.1 (for previous $Q^{4}$ results in the baryon octet sector see e.g. [12, 30], some partial results in the baryon decuplet sector can be found in [87]). Collecting all together, it holds:

$$
\begin{aligned}
& \Sigma_{B \in[8]}^{\text {loop }}=-\sum_{Q \in[8], R \in[8]}\left(\frac{G_{Q R}^{(B)}}{2 f}\right)^{2}\left[\frac{\left(M_{B}+M_{R}\right)^{2}}{E_{R}+M_{R}} p_{Q R}^{2}\left(\bar{I}_{Q R}+\frac{\bar{I}_{Q}}{M_{R}^{2}-m_{Q}^{2}}\right)-\frac{M_{R}^{2}-M_{B}^{2}}{2 M_{B}} \bar{I}_{Q}\right] \\
& -\sum_{Q \in[8], R \in[10]}\left(\frac{G_{Q R}^{(B)}}{2 f}\right)^{2}\left[\frac{2}{3} \frac{M_{B}^{2}}{M_{R}^{2}}\left(E_{R}+M_{R}\right) p_{Q R}^{2}\left(\bar{I}_{Q R}+\frac{\bar{I}_{Q}}{M_{R}^{2}-m_{Q}^{2}}\right)\right. \\
& \left.-\frac{1}{12 M_{B} M_{R}^{2}}\left(m_{Q}^{4}+\left(5 M_{B}^{2}+6 M_{R} M_{B}-2 M_{R}^{2}\right) m_{Q}^{2}+\left(M_{R}+M_{B}\right)^{3}\left(M_{R}-M_{B}\right)\right) \bar{I}_{Q}\right]
\end{aligned}
$$

\footnotetext{
${ }^{3}$ According to the power counting, the leading term in the one-loop contribution calculated with (1.45) is of the third chiral order. Within a chiral loop expansion a partial summation of higher order terms is performed. As a matter of simpler bookkeeping, the whole loop is labeled as $Q^{3}$.

${ }^{4}$ In [79] the dependence of the baryon self-energy on the off-shell parameter $Z$ for the spin-3/2 fields was considered. This dependence can be fully absorbed in the contact terms considered here and is not taken into account in this work. More details on the redundancy of the off-shell parameter in effective field theories can be found in Appendix A.4.
} 


$$
\begin{aligned}
& +\frac{1}{(2 f)^{2}} \sum_{Q \in[8]}\left(G_{B Q}^{(\chi)}-G_{B Q}^{(S)} m_{Q}^{2}-G_{B Q}^{(V)} \frac{1}{4} M_{B} m_{Q}^{2}\right) \bar{I}_{Q} \\
& \sum_{B \in[10]}^{\text {loop }}=-\sum_{Q \in[8], R \in[8]}\left(\frac{G_{Q R}^{(B)}}{2 f}\right)^{2}\left[\frac{1}{3}\left(E_{R}+M_{R}\right) p_{Q R}^{2}\left(\bar{I}_{Q R}+\frac{\bar{I}_{Q}}{M_{R}^{2}-m_{Q}^{2}}\right)\right. \\
& \left.-\frac{1}{24 M_{B}^{3}}\left(m_{Q}^{4}-\left(3 M_{B}^{2}+2 M_{R} M_{B}+2 M_{R}^{2}\right) m_{Q}^{2}+\left(M_{R}+M_{B}\right)^{3}\left(M_{R}-M_{B}\right)\right) \bar{I}_{Q}\right] \\
& -\sum_{Q \in[8], R \in[10]}\left(\frac{G_{Q R}^{(B)}}{2 f}\right)^{2}\left[\frac{\left(M_{B}+M_{R}\right)^{2}}{9 M_{R}^{2}} \frac{2 E_{R}\left(E_{R}-M_{R}\right)+5 M_{R}^{2}}{E_{R}+M_{R}} p_{Q R}\left(\bar{I}_{Q R}\left(M_{B}^{2}\right)+\frac{\bar{I}_{Q}}{M_{R}^{2}-m_{Q}^{2}}\right)\right. \\
& -\frac{1}{36 M_{B}^{3} M_{R}^{2}}\left(\left(M_{B}+M_{R}\right)^{2} m_{Q}^{4}+\left(3 M_{B}^{4}-2 M_{B}^{3} M_{R}+3 M_{B}^{2} M_{R}^{2}-2 M_{R}^{4}\right) m_{Q}^{2}\right. \\
& \left.\left.+\left(M_{R}^{4}+M_{B}^{4}+12 M_{R}^{2} M_{B}^{2}-2 M_{R} M_{B}\left(M_{B}^{2}+M_{R}^{2}\right)\right)\left(M_{R}^{2}-M_{B}^{2}\right)\right) \bar{I}_{Q}\right] \\
& +\frac{1}{(2 f)^{2}} \sum_{Q \in[8]}\left(G_{B Q}^{(\chi)}-G_{B Q}^{(S)} m_{Q}^{2}-G_{B Q}^{(V)} \frac{1}{4} M_{B} m_{Q}^{2}\right) \bar{I}_{Q},
\end{aligned}
$$

with

$$
\begin{aligned}
\bar{I}_{Q} & =\frac{m_{Q}^{2}}{(4 \pi)^{2}} \ln \left(\frac{m_{Q}^{2}}{\mu_{U V}^{2}}\right) \\
\bar{I}_{Q R} & =\frac{1}{(4 \pi)^{2}}\left[\frac{2 \pi \mu_{I R}}{\stackrel{\circ}{M}}+\left(\frac{1}{2} \frac{m_{Q}^{2}+M_{R}^{2}}{m_{Q}^{2}-M_{R}^{2}}-\frac{m_{Q}^{2}-M_{R}^{2}}{2 M_{B}^{2}}\right) \ln \left(\frac{m_{Q}^{2}}{M_{R}^{2}}\right)\right. \\
& \left.+\frac{p_{Q R}}{M_{B}}\left(\ln \left(1-\frac{M_{B}^{2}-2 p_{Q R} M_{B}}{m_{Q}^{2}+M_{R}^{2}}\right)-\ln \left(1-\frac{M_{B}^{2}+2 p_{Q R} M_{B}}{m_{Q}^{2}+M_{R}^{2}}\right)\right)\right] \\
p_{Q R}^{2} & =\frac{M_{B}^{2}}{4}-\frac{M_{R}^{2}+m_{Q}^{2}}{2}+\frac{\left(M_{R}^{2}-m_{Q}^{2}\right)^{2}}{4 M_{B}^{2}}, \quad E_{R}^{2}=M_{R}^{2}+p_{Q R}^{2} .
\end{aligned}
$$

The sums in $(3.3,3.4)$ extend over the intermediate Goldstone bosons $(Q \in[8])$, baryon octet $(R \in[8])$ and decuplet states $(R \in[10])$. The coupling constants $G_{Q R}^{(B)}$ are determined by the parameters $F, D, C$ and $H$. They are listed in Table 3.2.

The coupling constants $G_{Q R}^{(\chi)}$ probe the symmetry breaking parameters $b_{0}, b_{D}, b_{F}, d_{0}$ and $d_{D}$. They are detailed in Table 3.4 together with $G_{Q R}^{(S)}$ and $G_{Q R}^{(V)}$ which are proportional to the symmetry preserving parameters introduced in Section 1.4.2. In Table 3.4 we apply the notation

$$
\tilde{h}_{1}^{(S)} \equiv h_{1}^{(S)}+\frac{1}{4} h_{2}^{(S)}, \quad \tilde{h}_{2}^{(S)} \equiv h_{3}^{(S)}+\frac{1}{4} h_{4}^{(S)}, \quad \tilde{h}_{3}^{(S)} \equiv h_{5}^{(S)}+\frac{1}{4} h_{6}^{(S)} .
$$

The 6 parameters $h_{1-6}^{(S)}$ enter the decuplet self energy in the three combinations (3.6) only. 
The ultraviolet and the infrared scales in the renormalised scalar integrals in (3.5) are associated with the divergences in the non-renormalised expressions in $d=4$ and in $d=3$, respectively. While divergencies at $d=4$ occur always, the $d=3$ singularities are only present in the limit of vanishing meson masses (or in the limit of infinitely heavy baryons). The renormalised mesonic tadpole integral $\bar{I}_{Q}$ in (3.5) has a logarithmic dependence on the ultraviolet renormalization scale $\mu_{U V}$. The one-loop scalar function $\bar{I}_{Q R}$ shows a linear dependence on the infrared renormalization scale $\mu_{I R}$. The infrared as well as the ultraviolet scale dependencies are canceled by the corresponding scale dependencies of the counter terms. By applying a chiral expansion to the results in $(3.3,3.4)$, the running of the chiral parameters on these scales can be worked out order by order. This procedure results in scale independent results for the observables. The scale dependence of the parameters was exemplified in [79] at order $Q^{3}$ for $b_{0}, b_{D}, b_{F}$ and $d_{0}, d_{D}$.

Here we generalise that result to order $Q^{4}$ and detail the dependence on the renormalization scales of all contact terms taken into account in our calculation.

We introduce renormalised coupling constants, $\bar{g}$, with

$$
\begin{aligned}
g=\bar{g}+\frac{1}{4} \frac{1}{(4 \pi f)^{2}} & {\left[\Gamma_{g}^{\mathrm{UV}}\left(\frac{2}{d-4}-\ln (4 \pi)-1+\gamma_{E}\right)-\Gamma_{g}^{\mathrm{fin} .}\right.} \\
+ & \left.\Gamma_{g}^{\mathrm{IR}}\left(\frac{1}{2}-\mu_{\mathrm{IR}} / \stackrel{\circ}{M}\right) \frac{1}{d-3}\right],
\end{aligned}
$$

where $g$ is a synonym for any of the bare coupling constants introduced in Section 1.4.3. The coefficients $\Gamma_{g}^{\mathrm{UV}}$ and $\Gamma_{g}^{\mathrm{IR}}$ specify the dependence on the ultraviolet and infrared renormalization scales $\mu_{\mathrm{UV}}$ and $\mu_{\mathrm{IR}}$. The coefficients $\Gamma_{g}^{\text {fin. }}$ stand for the renormalization through finite terms arising in the limiting process $d \rightarrow 4$ in the dimensional regularisation. Those are collected in Appendix A.3.

Table 3.1 contains $\Gamma_{g}^{\mathrm{UV}}, \Gamma_{g}^{\mathrm{IR}}$ and $\Gamma_{g}^{\mathrm{fin}}$. for the $Q^{2}$ and $Q^{3}$ symmetry breaking couplings. The analogous expressions for the $Q^{4}$ couplings are given in A.2 and A.3. The coefficients are expanded in powers of

$$
\Delta=\stackrel{\circ}{M}_{[10]}-\stackrel{\circ}{M}_{[8]},
$$

and only the leading term in this expansion is shown.

Keeping the partial summation as defined by $(3.3,3.4)$ and truncating the contributions from the symmetry breaking part of the Lagrangian at $Q^{4}$, a residual dependence on the renormalization scales remains. This is analogous to the residual cutoff dependence of the scheme of Donoghue and Holstein [25]. As long as such dependencies are small and decreasing as higher order terms are included they do not pose a problem, rather, they offer a convenient way to estimate the error encountered at a given truncation.

Convergence properties of a perturbative expansion depend on the choice of the renormalization scales. Good convergence properties can only be expected for natural values of them. The ultraviolet scale makes integrals converge for virtual momenta beyond this scale. We use $\mu_{U V}=800 \mathrm{MeV}$. For the infrared scale $\mu_{I R}$ a natural window $350 \mathrm{MeV}$ $<\mu_{I R}<550 \mathrm{MeV}$ was suggested in [79]. This proposal is justified by following considerations. The leading chiral contribution to the baryon mass in the addend of (3.3) is 


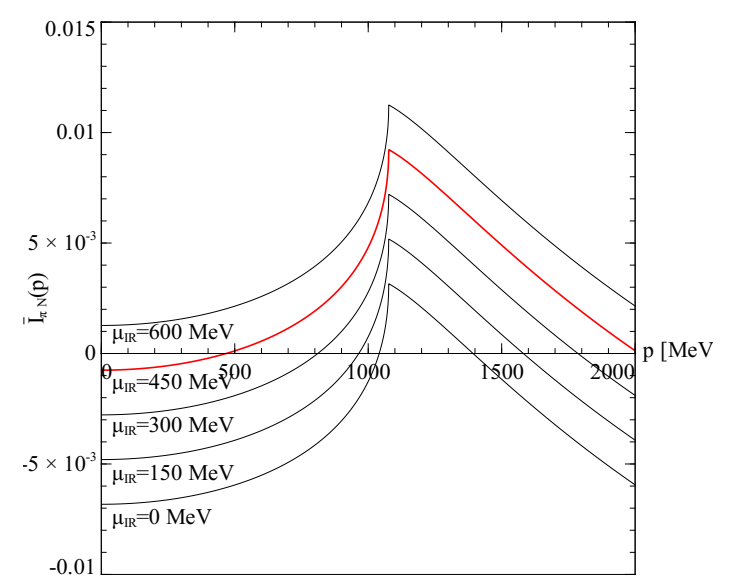

(a)

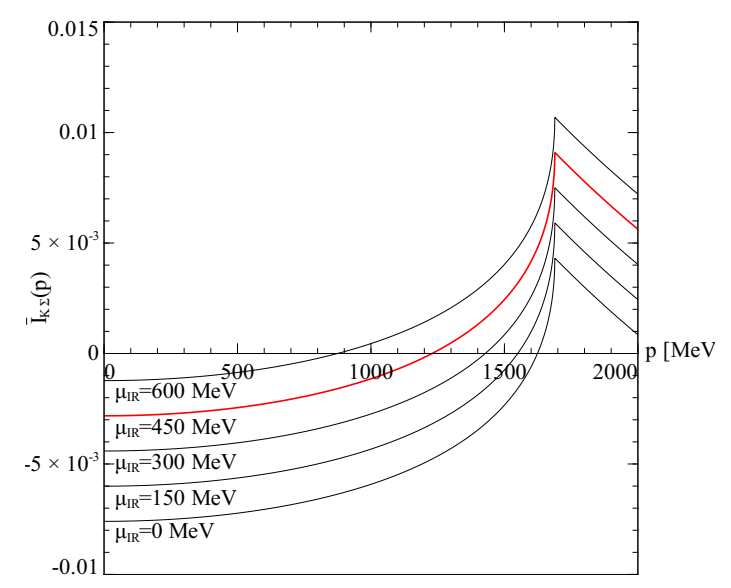

(b)

Figure 3.1.: $\bar{I}_{\pi N}(p)$ and $\bar{I}_{K \Sigma}(p)$ for different choices of the infrared scale $\mu_{I R}$.

proportional to

$$
m_{Q}^{2} \bar{I}_{Q R}\left(M_{R}\right)=m_{Q}^{2}\left(\frac{1}{8 \pi^{2}} \frac{\mu_{I R}}{M_{R}}-\frac{1}{16 \pi} \frac{m_{Q}}{M_{R}}+\mathcal{O}\left(Q^{2}\right)\right)
$$

For $\mu_{I R}=0 \mathrm{MeV}$ the leading order term is $\sim m_{Q}^{3}$ and the mass shift is dominated by the kaon and eta mesons ${ }^{5}$. This is in contradiction to the physical expectation that the low-energy expansion should be governed by the lighter degrees of freedom. Values of $\mu_{I R}$ closer to the heavier meson masses cancel a big part of their contributions and make the pion-contribution more dominant.

This situation is not changed by the higher order terms in the one-loop function which were omitted in the expansion on the r.h.s. of (3.9). In Figure 3.1 the loop-functions $\bar{I}_{\pi N}(p)$ and $\bar{I}_{K \Sigma}(p)$ for different choices of the infrared scale $\mu_{I R}$ are shown. E.g., for the nucleon self energy these loop integrals have to be evaluated at $p \approx 1 \mathrm{GeV}$. As can be seen in Figure 3.1, for $\mu_{I R}=450 \mathrm{MeV}$, which is used in this work, the contribution of the heavier kaon-mass is strongly suppressed as compared to the pion-loop.

We turn to the tree-level contributions to the baryon masses. The unrenormalised expressions were already specified in Section 1.4.3. The renormalised expressions are obtained by replacing the couplings in $(1.71,1.72)$ with their renormalised values.

Note, that in our scheme there are no finite terms induced by the dimensional regularised loop integrals in the limit $d \rightarrow 4$. Such terms carry minimal chiral power $Q^{3}$, define a finite shift to the symmetry breaking parameters and are completely absorbed in the definition of the renormalised couplings in (3.7).

\footnotetext{
${ }^{5} \mathrm{~A}$ baryon mass shift proportional to the third power of meson masses is the result of the strict chiral expansion as in heavy baryon calculations. For three quark flavours, this huge loop-correction spoils the convergence pattern.
} 


\begin{tabular}{|c|c|c|c|}
\hline & $\Gamma^{\mathrm{UV}}$ & $\Gamma^{\mathrm{IR}} / \stackrel{\circ}{M}$ & $\Gamma^{\text {fin. }}$ \\
\hline$b_{0}$ & $\frac{7}{9} C^{2} \Delta$ & $\frac{4}{9}\left(13 D^{2}+9 F^{2}+7 C^{2}\right)$ & $\frac{7}{27} C^{2} \Delta$ \\
\hline$b_{D}$ & $-\frac{1}{3} C^{2} \Delta$ & $-\frac{2}{3}\left(3 D^{2}-9 F^{2}+2 C^{2}\right)$ & $-\frac{1}{9} C^{2} \Delta$ \\
\hline$b_{F}$ & $\frac{5}{18} C^{2} \Delta$ & $\frac{10}{9}\left(6 D F+C^{2}\right)$ & $\frac{5}{54} C^{2} \Delta$ \\
\hline$d_{0}$ & $-\frac{1}{6} C^{2} \Delta$ & $\frac{2}{81}\left(27 C^{2}+25 H^{2}\right)$ & $\frac{1}{9} C^{2} \Delta$ \\
\hline$d_{D}$ & $-\frac{1}{6} C^{2} \Delta$ & $\frac{2}{9}\left(3 C^{2}+5 H^{2}\right)$ & $\frac{1}{9} C^{2} \Delta$ \\
\hline$\zeta_{0}$ & $-\frac{26}{9} D^{2}-2 F^{2}-\frac{14}{9} C^{2}$ & $\begin{array}{l}\frac{56}{9} C^{2} \stackrel{\circ}{M}_{[8]}^{-1}-\frac{32}{9}\left(2 D^{2}\left(15 b_{0}+13 b_{D}\right)\right. \\
\left.\quad+9 F^{2}\left(6 b_{0}+2 b_{D}\right)+36 D F b_{F}+3 C^{2}\left(5 d_{0}+d_{D}\right)\right)\end{array}$ & $-\frac{14}{27} C^{2}$ \\
\hline$\zeta_{D}$ & $D^{2}-3 F^{2}+\frac{2}{3} C^{2}$ & $-\frac{8}{3} C^{2} \stackrel{\circ}{M}_{[8]}^{-1}+\frac{32}{3}\left(3 D^{2} b_{D}-9 F^{2} b_{D}+18 D F b_{F}-C^{2} d_{D}\right)$ & $\frac{2}{9} C^{2}$ \\
\hline$\zeta_{F}$ & $-\frac{10}{3} D F-\frac{5}{9} C^{2}$ & $\frac{20}{9} C^{2} \stackrel{\circ}{M}_{[8]}^{-1}-\frac{32}{3}\left(15 D^{2} b_{F}+27 F^{2} b_{F}-30 D F b_{D}+5 C^{2} d_{D}\right)$ & $-\frac{5}{27} C^{2}$ \\
\hline$\xi_{0}$ & $-\frac{1}{3} C^{2}-\frac{25}{81} H^{2}$ & $-\frac{4}{3} C^{2} \stackrel{\circ}{M}_{[8]}^{-1}-\frac{32}{81}\left(27 C^{2}\left(2 b_{0}+b_{D}-b_{F}\right)+5 H^{2}\left(12 d_{0}+d_{D}\right)\right)$ & $\frac{2}{9} C^{2}-\frac{80}{243} H^{2}$ \\
\hline$\xi_{D}$ & $-\frac{1}{3} C^{2}-\frac{5}{9} H^{2}$ & $-\frac{4}{3} C^{2} \stackrel{\circ}{M}_{[8]}^{-1}-\frac{32}{9}\left(3 C^{2}\left(b_{D}+3 b_{F}\right)+5 H^{2} d_{D}\right)$ & $\frac{2}{9} C^{2}-\frac{16}{27} H^{2}$ \\
\hline
\end{tabular}

Table 3.1.: Renormalisation coefficients of the $Q^{2}$ and $Q^{3}$ symmetry breaking parameters (see $(3.7))$.

\begin{tabular}{|l|l|l|l|}
\hline$G_{\pi N}^{(N)}=\sqrt{3}(D+F)$ & $G_{\pi \Sigma}^{(\Lambda)}=2 D$ & $G_{\pi \Lambda}^{(\Sigma)}=\frac{2 D}{\sqrt{3}}$ & $G_{\pi \Xi}^{(\Xi)}=-\sqrt{3}(D-F)$ \\
$G_{\eta N}^{(N)}=-\frac{D-3 F}{\sqrt{3}}$ & $G_{\bar{K} N}^{(\Lambda)}=-\sqrt{\frac{2}{3}}(D+3 F)$ & $G_{\pi \Sigma}^{(\Sigma)}=-\sqrt{8} F$ & $G_{\bar{K} \Lambda}^{(\Xi)}=-\frac{D-3 F}{\sqrt{3}}$ \\
$G_{K \Lambda}^{(N)}=-\frac{D+3 F}{\sqrt{3}}$ & $G_{\eta \Lambda}^{(\Lambda)}=-\frac{2 D}{\sqrt{3}}$ & $G_{\bar{K} N}^{(\Sigma)}=\sqrt{2}(D-F)$ & $G_{\bar{K} \Sigma}^{(\Xi)}=-\sqrt{3}(D+F)$ \\
$G_{K \Sigma}^{(N)}=\sqrt{3}(D-F)$ & $G_{K \Xi}^{(\Lambda)}=\sqrt{\frac{2}{3}}(D-3 F)$ & $G_{\eta \Sigma}^{(\Sigma)}=\frac{2 D}{\sqrt{3}}$ & $G_{\eta \Xi}^{(\Xi)}=-\frac{D+3 F}{\sqrt{3}}$ \\
& & $G_{K \Xi}^{(\Sigma)}=\sqrt{2}(D+F)$ & \\
\hline$G_{\pi \Delta}^{(N)}=2 C$ & $G_{\pi \Sigma}^{(\Lambda)}=-\sqrt{3} C$ & $G_{\pi \Sigma}^{(\Sigma)}=-\sqrt{\frac{2}{3}} C$ & $G_{\pi \Xi}^{(\Xi)}=-C$ \\
$G_{K \Sigma}^{(N)}=C$ & $G_{K \Xi}^{(\Lambda)}=-\sqrt{2} C$ & $G_{\bar{K} \Delta}^{(\Sigma)}=-\sqrt{\frac{8}{3}} C$ & $G_{\eta \Xi}^{(\Xi)}=-C$ \\
& & $G_{\eta \Sigma}^{(\Sigma)}=C$ & $G_{\bar{K} \Sigma}^{(\Xi)}=C$ \\
& & $G_{K \Xi}^{(\Sigma)}=-\sqrt{\frac{2}{3}} C$ & $G_{K \Omega}^{(\Xi)}=-\sqrt{2} C$ \\
\hline
\end{tabular}

Table 3.2.: Meson-baryon coupling constants $G_{Q R}^{(B)}$ with $B \in[8]$.

The results $(3.3,3.4)$ depend on the physical meson and baryon masses $m_{Q}$ and $M_{R}$. This defines a self consistent summation since the masses of the intermediate baryon states in $(3.3,3.4)$ should match the total masses. The baryon masses are a solution of a set of eight coupled and non-linear equations in (3.2). This is a consequence of self consistency imposed on the partial summation approach. The latter is a crucial requirement since the loop functions depend sensitively on the precise values of the baryon masses. 


\begin{tabular}{|l|l|l|l|}
\hline$G_{\pi N}^{(\Delta)}=\sqrt{2} C$ & $G_{\pi \Lambda}^{(\Sigma)}=-C$ & $G_{\pi \Xi}^{(\Xi)}=-C$ & $G_{\bar{K} \Xi}^{(\Omega)}=-2 C$ \\
$G_{K \Sigma}^{(\Delta)}=-\sqrt{2} C$ & $G_{\pi \Sigma}^{(\Sigma)}=-\sqrt{\frac{2}{3}} C$ & $G_{\bar{K} \Lambda}^{(\Xi)}=C$ & \\
& $G_{K N}^{(\Sigma)}=\sqrt{\frac{2}{3}} C$ & $G_{\bar{K} \Sigma}^{(\Xi)}=C$ & \\
& $G_{\eta \Sigma}^{(\Sigma)}=C$ & $G_{\eta \Xi}^{(\Xi)}=-C$ & \\
& $G_{K \Xi}^{(\Sigma)}=-\sqrt{\frac{2}{3}} C$ & & \\
\hline$G_{\pi \Delta}^{(\Delta)}=-\sqrt{\frac{5}{3}} H$ & $G_{\pi \Sigma}^{(\Sigma)}=\frac{\sqrt{8}}{3} H$ & $G_{\pi \Xi}^{(\Xi)}=-\sqrt{\frac{1}{3}} H$ & $G_{K \Xi}^{(\Omega)}=-\frac{2}{\sqrt{3}} H$ \\
$G_{\eta \Delta}^{(\Delta)}=-\sqrt{\frac{1}{3}} H$ & $G_{\bar{K}}^{(\Sigma)}=-\frac{\sqrt{8}}{3} H$ & $G_{\bar{K} \Sigma}^{(\Xi)}=-\frac{2}{\sqrt{3}} H$ & $G_{\eta \Omega}^{(\Omega)}=\frac{2}{\sqrt{3}} H$ \\
$G_{K \Sigma}^{(\Delta)}=-\sqrt{\frac{2}{3}} H$ & $G_{\eta \Sigma}^{(\Sigma)}=0$ & $G_{K \Omega}^{(\Xi)}=-\sqrt{\frac{2}{3}} H$ & \\
& $G_{K \Xi}^{(\Sigma)}=\frac{\sqrt{8}}{3} H$ & $G_{\eta \Xi}^{(\Xi)}=\frac{1}{\sqrt{3}} H$ & \\
\hline
\end{tabular}

Table 3.3.: Meson-baryon coupling constants $G_{Q R}^{(B)}$ with $B \in[10]$.

\subsection{Chiral extrapolation for the light hadrons}

The dependence of the baryon masses on the masses of the light quarks enters into the calculation, once the explicit chiral symmetry breaking is taken into account. Besides the tree-level contributions to the baryon masses, the dependence on the quark masses is furthermore determined trough the non-vanishing meson masses in the loops. We discuss the quark mass dependence of the mesons first.

\subsubsection{Meson masses}

To the fourth chiral order the meson masses are determined by solving the equation $([37,78])$

$$
m_{Q}^{2}=m_{Q, 2}^{2}+\Sigma\left(m_{Q}^{2}\right) \equiv m_{Q, 2}^{2}+A_{Q}+B_{Q} m_{Q}^{2}, \quad Q \in[8],
$$

with the $Q^{2}$-values

$$
m_{\pi, 2}^{2}=2 B_{0} \hat{m}, \quad m_{K, 2}^{2}=B_{0}\left(\hat{m}+m_{s}\right), \quad m_{\eta, 2}^{2}=\frac{2}{3} B_{0}\left(\hat{m}+2 m_{s}\right),
$$

and

$$
\begin{aligned}
& A_{\pi}=\frac{m_{\pi}^{2}}{f^{2}}\left\{-\frac{1}{6} \bar{I}_{\pi}-\frac{1}{6} \bar{I}_{\eta}-\frac{1}{3} \bar{I}_{K}+32\left[\left(2 \hat{m}+m_{s}\right) B_{0} L_{6}+\hat{m} B_{0} L_{8}\right]\right\}, \\
& B_{\pi}=\frac{2}{3} \frac{\bar{I}_{\pi}}{f^{2}}+\frac{1}{3} \frac{\bar{I}_{K}}{f^{2}}-\frac{16 B_{0}}{f^{2}}\left[\left(2 \hat{m}+m_{s}\right) L_{4}+\hat{m} L_{5}\right], \\
& A_{K}=\frac{m_{K}^{2}}{f^{2}}\left\{\frac{1}{12} \bar{I}_{\eta}-\frac{1}{4} \bar{I}_{\pi}-\frac{1}{2} \bar{I}_{K}+32\left[\left(2 \hat{m}+m_{s}\right) B_{0} L_{6}+\frac{1}{2}\left(\hat{m}+m_{s}\right) B_{0} L_{8}\right]\right\},
\end{aligned}
$$




\begin{tabular}{|c|c|c|c|c|}
\hline$B$ & $Q$ & $G_{B Q}^{(\chi)}$ & $G_{B Q}^{(S)}$ & $G_{B Q}^{(V)}$ \\
\hline$N$ & $K$ & $\begin{array}{c}24 B_{0} m\left(2 \bar{b}_{0}+\bar{b}_{D}+\bar{b}_{F}\right) \\
8 B_{0}\left(m+m_{s}\right)\left(4 \bar{b}_{0}+3 \bar{b}_{D}-\bar{b}_{F}\right) \\
\frac{8}{3} B_{0} m\left(2 \bar{b}_{0}+\bar{b}_{D}+\bar{b}_{F}\right) \\
+\frac{32}{3} B_{0} m_{s}\left(\bar{b}_{0}+\bar{b}_{D}-\bar{b}_{F}\right)\end{array}$ & $\begin{array}{c}3 g_{0}^{(S)}+\frac{3}{2} g_{D}^{(S)}+\frac{3}{2} g_{F}^{(S)} \\
4 g_{0}^{(S)}+g_{1}^{(S)}+3 g_{D}^{(S)}-g_{F}^{(S)} \\
g_{0}^{(S)}+\frac{5}{6} g_{D}^{(S)}-\frac{1}{2} g_{F}^{(S)}\end{array}$ & $\begin{array}{c}3 g_{0}^{(V)}+\frac{3}{2} g_{D}^{(V)}+\frac{3}{2} g_{F}^{(V)} \\
4 g_{0}^{(V)}+g_{1}^{(V)}+3 g_{D}^{(V)}-g_{F}^{(V)} \\
g_{0}^{(V)}+\frac{5}{6} g_{D}^{(V)}-\frac{1}{2} g_{F}^{(V)}\end{array}$ \\
\hline$\Lambda$ & $K$ & $\begin{array}{c}16 B_{0} m\left(3 \bar{b}_{0}+\bar{b}_{D}\right) \\
\frac{16}{3} B_{0}\left(m+m_{s}\right)\left(6 \bar{b}_{0}+5 \bar{b}_{D}\right) \\
\frac{16}{9} B_{0} m\left(3 \bar{b}_{0}+\bar{b}_{D}\right) \\
\quad+\frac{32}{9} B_{0} m_{s}\left(3 \bar{b}_{0}+4 \bar{b}_{D}\right)\end{array}$ & $\begin{array}{c}3 g_{0}^{(S)}+g_{D}^{(S)} \\
4 g_{0}^{(S)}+\frac{10}{3} g_{D}^{(S)} \\
g_{0}^{(S)}+g_{1}^{(S)}+g_{D}^{(S)}\end{array}$ & $\begin{array}{c}3 g_{0}^{(V)}+g_{D}^{(V)} \\
4 g_{0}^{(V)}+\frac{10}{3} g_{D}^{(V)} \\
g_{0}^{(V)}+g_{1}^{(V)}+g_{D}^{(V)}\end{array}$ \\
\hline$\Sigma$ & $\eta$ & $\begin{array}{c}48 B_{0} m\left(\bar{b}_{0}+\bar{b}_{D}\right) \\
16 B_{0}\left(m+m_{s}\right)\left(2 \bar{b}_{0}+\bar{b}_{D}\right) \\
\frac{16}{3} B_{0}\left(m\left(\bar{b}_{0}+\bar{b}_{D}\right)+2 m_{s} \bar{b}_{0}\right)\end{array}$ & $\begin{array}{c}3 g_{0}^{(S)}+g_{1}^{(S)}+3 g_{D}^{(S)} \\
4 g_{0}^{(S)}+2 g_{D}^{(S)} \\
g_{0}^{(S)}+\frac{1}{3} g_{D}^{(S)}\end{array}$ & $\begin{array}{c}3 g_{0}^{(V)}+g_{1}^{(V)}+3 g_{D}^{(V)} \\
4 g_{0}^{(V)}+2 g_{D}^{(V)} \\
g_{0}^{(V)}+\frac{1}{3} g_{D}^{(V)}\end{array}$ \\
\hline$\Xi$ & $K$ & $\begin{array}{c}24 B_{0} m\left(2 \bar{b}_{0}+\bar{b}_{D}-\bar{b}_{F}\right) \\
8 B_{0}\left(m+m_{s}\right)\left(4 \bar{b}_{0}+3 \bar{b}_{D}+\bar{b}_{F}\right) \\
\frac{8}{3} B_{0} m\left(2 \bar{b}_{0}+\bar{b}_{D}-\bar{b}_{F}\right) \\
\quad+\frac{32}{3} B_{0} m_{s}\left(\bar{b}_{0}+\bar{b}_{D}+\bar{b}_{F}\right)\end{array}$ & $\begin{array}{c}3 g_{0}^{(S)}+\frac{3}{2} g_{D}^{(S)}-\frac{3}{2} g_{F}^{(S)} \\
4 g_{0}^{(S)}+g_{1}^{(S)}+3 g_{D}^{(S)}+g_{F}^{(S)} \\
g_{0}^{(S)}+\frac{5}{6} g_{D}^{(S)}+\frac{1}{2} g_{F}^{(S)}\end{array}$ & $\begin{array}{c}3 g_{0}^{(V)}+\frac{3}{2} g_{D}^{(V)}-\frac{3}{2} g_{F}^{(V)} \\
4 g_{0}^{(V)}+g_{1}^{(V)}+3 g_{D}^{(V)}+g_{F}^{(V)} \\
g_{0}^{(V)}+\frac{5}{6} g_{D}^{(V)}+\frac{1}{2} g_{F}^{(V)}\end{array}$ \\
\hline$\Delta$ & $\eta$ & $\begin{array}{c}24 B_{0} m\left(2 \bar{d}_{0}+\bar{d}_{D}\right) \\
8 B_{0}\left(m+m_{s}\right)\left(4 \bar{d}_{0}+\bar{d}_{D}\right) \\
\frac{8}{3} B_{0}\left(m\left(2 \bar{d}_{0}+\bar{d}_{D}\right)+2 m_{s} \bar{d}_{0}\right)\end{array}$ & $\begin{array}{c}3 \tilde{h}_{1}^{(S)}+3 \tilde{h}_{2}^{(S)}+2 \tilde{h}_{3}^{(S)} \\
4 \tilde{h}_{1}^{(S)}+2 \tilde{h}_{2}^{(S)}+2 \tilde{h}_{3}^{(S)} \\
\tilde{h}_{1}^{(S)}+\frac{1}{3} \tilde{h}_{2}^{(S)}\end{array}$ & $\begin{array}{c}3 h_{1}^{(V)}+3 h_{2}^{(V)}+2 h_{3}^{(V)} \\
4 h_{1}^{(V)}+2 h_{2}^{(V)}+2 h_{3}^{(V)} \\
h_{1}^{(V)}+\frac{1}{3} h_{2}^{(V)}\end{array}$ \\
\hline$\Sigma^{*}$ & $\eta$ & $\begin{array}{c}16 B_{0} m\left(3 \bar{d}_{0}+\bar{d}_{D}\right) \\
\frac{32}{3} B_{0}\left(m+m_{s}\right)\left(3 \bar{d}_{0}+\bar{d}_{D}\right) \\
\frac{16}{9} B_{0}\left(m+2 m_{s}\right)\left(3 \bar{d}_{0}+\bar{d}_{D}\right)\end{array}$ & $\begin{array}{c}3 \tilde{h}_{1}^{(S)}+2 \tilde{h}_{2}^{(S)}+\frac{5}{3} \tilde{h}_{3}^{(S)} \\
4 \tilde{h}_{1}^{(S)}+\frac{8}{3} \tilde{h}_{2}^{(S)}+\frac{4}{3} \tilde{h}_{3}^{(S)} \\
\tilde{h}_{1}^{(S)}+\frac{2}{3} \tilde{h}_{2}^{(S)}+\tilde{h}_{3}^{(S)}\end{array}$ & $\begin{array}{c}3 h_{1}^{(V)}+2 h_{2}^{(V)}+\frac{5}{3} h_{3}^{(V)} \\
4 h_{1}^{(V)}+\frac{8}{3} h_{2}^{(V)}+\frac{4}{3} h_{3}^{(V)} \\
h_{1}^{(V)}+\frac{2}{3} h_{2}^{(V)}+h_{3}^{(V)}\end{array}$ \\
\hline$\Xi^{*}$ & $K$ & $\begin{array}{c}8 B_{0} m\left(6 \bar{d}_{0}+\bar{d}_{D}\right) \\
\frac{8}{3} B_{0}\left(m+m_{s}\right)\left(12 \bar{d}_{0}+5 \bar{d}_{D}\right) \\
\frac{8}{9} B_{0} m\left(6 \bar{d}_{0}+\bar{d}_{D}\right) \\
\quad+\frac{8}{9} B_{0} m_{s}\left(12 \bar{d}_{0}+8 \bar{d}_{D}\right)\end{array}$ & $\begin{array}{c}3 \tilde{h}_{1}^{(S)}+\tilde{h}_{2}^{(S)}+\tilde{h}_{3}^{(S)} \\
4 \tilde{h}_{1}^{(S)}+\frac{10}{3} \tilde{h}_{2}^{(S)}+2 \tilde{h}_{3}^{(S)} \\
\tilde{h}_{1}^{(S)}+\tilde{h}_{2}^{(S)}+\tilde{h}_{3}^{(S)}\end{array}$ & $\begin{array}{c}3 h_{1}^{(V)}+h_{2}^{(V)}+h_{3}^{(V)} \\
4 h_{1}^{(V)}+\frac{10}{3} h_{2}^{(V)}+2 h_{3}^{(V)} \\
h_{1}^{(V)}+h_{2}^{(V)}+h_{3}^{(V)}\end{array}$ \\
\hline$\Omega$ & $K$ & $\begin{array}{c}48 B_{0} m \bar{d}_{0} \\
16 B_{0}\left(m+m_{s}\right)\left(2 \bar{d}_{0}+\bar{d}_{D}\right) \\
\frac{16}{3} B_{0}\left(m \bar{d}_{0}+2 m_{s}\left(\bar{d}_{0}+\bar{d}_{D}\right)\right)\end{array}$ & $\begin{array}{c}3 \tilde{h}_{1}^{(S)} \\
4 \tilde{h}_{1}^{(S)}+4 \tilde{h}_{2}^{(S)}+4 \tilde{h}_{3}^{(S)} \\
\tilde{h}_{1}^{(S)}+\frac{4}{3} \tilde{h}_{2}^{(S)}\end{array}$ & $\begin{array}{c}3 h_{1}^{(V)} \\
4 h_{1}^{(V)}+4 h_{2}^{(V)}+4 h_{3}^{(V)} \\
h_{1}^{(V)}+\frac{4}{3} h_{2}^{(V)}\end{array}$ \\
\hline
\end{tabular}

Table 3.4.: Coefficients $G_{B Q}^{(\chi)}, G_{B Q}^{(S)}$ and $G_{B Q}^{(V)}$. 


$$
\begin{aligned}
B_{K} & =\frac{1}{4} \frac{\bar{I}_{\eta}}{f^{2}}+\frac{1}{4} \frac{\bar{I}_{\pi}}{f^{2}}+\frac{1}{2} \frac{\bar{I}_{K}}{f^{2}}-16 \frac{B_{0}}{f^{2}}\left[\left(2 \hat{m}+m_{s}\right) L_{4}+\frac{1}{2}\left(\hat{m}+m_{s}\right) L_{5}\right] \\
A_{\eta} & =\frac{m_{\eta}^{2}}{f^{2}}\left[-\frac{2}{3} \bar{I}_{\eta}\right]+\frac{M_{\pi}^{2}}{f^{2}}\left[\frac{1}{6} \bar{I}_{\eta}-\frac{1}{2} \bar{I}_{\pi}+\frac{1}{3} \bar{I}_{K}\right]+\frac{128}{9} \frac{B_{0}^{2}\left(\hat{m}-m_{s}\right)^{2}}{f^{2}}\left(3 L_{7}+L_{8}\right) \\
& +\frac{m_{\eta}^{2}}{f^{2}}\left[16 \frac{2}{3}\left(\hat{m}+2 m_{s}\right) B_{0} L_{8}+32\left(2 \hat{m}+m_{s}\right) B_{0} L_{6}\right] \\
B_{\eta} & =\frac{\bar{I}_{K}}{f^{2}}-\frac{16}{f^{2}}\left(2 \hat{m}+m_{s}\right) B_{0} L_{4}-\frac{8}{f^{2}} \frac{2}{3}\left(\hat{m}+2 m_{s}\right) B_{0} L_{5} .
\end{aligned}
$$

Solving for the meson mass $m_{Q}$ in (3.10) yields

$$
m_{Q}^{2}=\frac{m_{Q, 2}^{2}+A_{Q}}{1-B_{Q}}=\underbrace{m_{Q, 2}^{2}\left(1+B_{Q}\right)+A_{Q}}_{\equiv m_{Q, 4}^{2}}+\mathcal{O}\left(Q^{6}\right),
$$

with the $Q^{4}$-values of the squared meson masses

$$
\begin{aligned}
m_{\pi, 4}^{2} & =m_{\pi, 2}^{2}\left\{1+\frac{m_{\pi, 2}^{2}}{32 \pi^{2} f^{2}} \ln \left(\frac{m_{\pi, 2}^{2}}{\mu_{U V}^{2}}\right)-\frac{m_{\eta, 2}^{2}}{96 \pi^{2} f^{2}} \ln \left(\frac{m_{\eta, 2}^{2}}{\mu_{U V}^{2}}\right)\right. \\
& \left.+\frac{16}{f^{2}}\left[\left(2 \hat{m}+m_{s}\right) B_{0}\left(2 L_{6}-L_{4}\right)+\hat{m} B_{0}\left(2 L_{8}-L_{5}\right)\right]\right\}, \\
m_{K, 4}^{2} & =m_{K, 2}^{2}\left\{1+\frac{m_{\eta, 2}^{2}}{48 \pi^{2} f^{2}} \ln \left(\frac{m_{\eta, 2}^{2}}{\mu_{U V}^{2}}\right)\right. \\
& \left.+\frac{16}{f^{2}}\left[\left(2 \hat{m}+m_{s}\right) B_{0}\left(2 L_{6}-L_{4}\right)+\frac{1}{2}\left(\hat{m}+m_{s}\right) B_{0}\left(2 L_{8}-L_{5}\right)\right]\right\} \\
m_{\eta, 4}^{2} & =m_{\eta, 2}^{2}\left[1+\frac{m_{K, 2}^{2}}{16 \pi^{2} f^{2}} \ln \left(\frac{m_{K, 2}^{2}}{\mu_{U V}^{2}}\right)-\frac{m_{\eta, 2}^{2}}{24 \pi^{2} f^{2}} \ln \left(\frac{m_{\eta, 2}^{2}}{\mu_{U V}^{2}}\right)\right. \\
& \left.+\frac{16}{f^{2}}\left(2 \hat{m}+m_{s}\right) B_{0}\left(2 L_{6}-L_{4}\right)+8 \frac{m_{\eta, 2}^{2}}{f^{2}}\left(2 L_{8}-L_{5}\right)\right] \\
& +m_{\pi, 2}^{2}\left[\frac{m_{\eta, 2}^{2}}{96 \pi^{2} f^{2}} \ln \left(\frac{m_{\eta, 2}^{2}}{\mu_{U V}^{2}}\right)-\frac{m_{\pi, 2}^{2}}{32 \pi^{2} f^{2}} \ln \left(\frac{m_{\pi, 2}^{2}}{\mu_{U V}^{2}}\right)+\frac{m_{K, 2}^{2}}{48 \pi^{2} f^{2}} \ln \left(\frac{m_{K, 2}^{2}}{\mu_{U V}^{2}}\right)\right] \\
& +\frac{128}{9} \frac{B_{0}^{2}\left(\hat{m}-m_{s}\right)^{2}}{f^{2}}\left(3 L_{7}+L_{8}\right) .
\end{aligned}
$$

These expressions are frequently used in the literature for the extrapolation of the meson masses.

Because of loop corrections, the renormalised values of the couplings $L_{4,5,6,8}$ are UV-scale dependent:

$$
L_{i}\left(\mu_{2}\right)=L_{i}\left(\mu_{1}\right)+\frac{\Gamma_{i}}{(4 \pi)^{2}} \ln \left(\frac{\mu_{1}^{2}}{\mu_{2}^{2}}\right), \quad i \in\{4,5,6,8\}
$$




\begin{tabular}{|c|c|}
\hline & Empirical value at $\mu_{U V}=0.8 \mathrm{GeV}$ \\
\hline $2 L_{6}-L_{4}$ & $-0.1 \times 10^{-3}$ \\
$2 L_{8}-L_{5}$ & $+0.4 \times 10^{-3}$ \\
$3 L_{7}+L_{8}$ & $-0.3 \times 10^{-3}$ \\
$m B_{0}$ & $9.91 \times 10^{-3} \mathrm{GeV}^{2}$ \\
$m_{s} B_{0}$ & $237 \times 10^{-3} \mathrm{GeV}^{2}$ \\
$f$ & $0.090 \mathrm{GeV}$ \\
\hline
\end{tabular}

Table 3.5.: Low-energy coupling constants used in this work.

The coefficients $\Gamma_{i}$ were first determined in [37]. The experimental values of $L_{i}$ given in [9] are converted according to (3.17) to the scale used in this work, $\mu_{U V}=800 \mathrm{MeV}$. The numerical values of them at this scale are presented in Table 3.5.

Instead of using the expressions in (3.14), we solve the general equation in (3.10). This amounts to a partial summation of higher order contributions from the symmetry breaking terms. Though, the difference between (3.14) and the solution of (3.10) is numerically negligible (as it should be in a well convergent expansion for an effect of higher order).

\subsubsection{Baryon masses}

The baryon masses are determined in the chiral loop expansion by a solution of a set of coupled and non-linear equations. For given values of the infrared and ultraviolet renormalization scales and the values of the light quark masses, the chiral parameters are determined by minimizing the function

$$
\chi^{2}=\sum_{B \in[8],[10]}\left(M_{B}-M_{B}^{\exp }\right) / M_{B}^{\exp }
$$

where $M_{B}$ 's are the solution of the coupled system (3.2), and the values of $M_{B}^{\exp }$ stand for the corresponding experimental values of the baryon masses.

Given the large number of chiral parameters at this order, it is possible to obtain an excellent value for $\chi^{2}$ in (3.18) for many different sets of the chiral parameters. The decomposition of the baryon masses in chiral moments serves as an exclusion criterion - from a convergent expansion one expects the higher order contributions to decrease in their absolute values. We exclude parameter sets that indicate no or poor convergence properties.

A further exclusion criterion is provided by the extrapolation of the results for the baryon masses in the quark masses. As a consequence of the non-linearity of the system, for a given set of parameters there is no guarantee for a unique solution to exist. Furthermore, 
given the set of chiral parameters which minimises (3.18) at the physical point, there is no guarantee for the corresponding baryon masses to possess a continuous quark mass dependence. The system may discontinuously evolve from one solution to another as the quark masses rise continuously. Indeed, various discontinuities in the quark-mass dependence of the baryon masses were reported in [80]. By including the $Q^{4}$ effects it is possible to find parameter sets leading to a unique (and, therefore, a stable) solution for the baryon masses.

A further constraint for the parameters is provided by lattice simulations of the baryon masses. In order to minimise the bias of these results on the chiral parameters, we only consider the values of the baryon masses for a single member of each baryon flavour multiplet at the $S U(3)$ symmetric point with $m=m_{s}$. This implies the following meson masses

$$
m_{\pi}=m_{K}=m_{\eta}=695.6 \mathrm{MeV} .
$$

The values at this point allows to disentangle various chiral parameters which occur at the physical point in specific combinations only ${ }^{6}$. This procedure increases the prediction power of the chiral extrapolation and the trust in $\chi \mathrm{PT}$ in the baryon sector outside the physical region, if the description of the baryon masses is also reasonable for higher values of the quark masses.

In the following we confront the chiral extrapolation of the baryon masses with the current results of various lattice groups working on the hadron spectrum in unquenched simulations with three light quarks $[4,88,1,62,27]$. We use the data of the BMW-group [27] for the nucleon and for the omega baryon. This group has carried out a very detailed study of the hadron spectrum at three different lattice spacings and provides continuum extrapolated results. For large quark masses the BMW-group did not provide yet results for other than the nucleon and the omega baryon. The value of the baryon masses at the $S U(3)$ symmetric point in (3.19), as implied by the smallest lattice spacing in the simulation of the BMW-collaboration, is

$$
M_{[8]} \approx 1560 \mathrm{MeV}, \quad M_{[10]} \approx 1768 \mathrm{MeV} .
$$

A possible scenario for the chiral parameters is summarised in Tables 3.6 and 3.7, which contain the values of all parameters entering the calculation of the baryon masses at $Q^{4}$. The chiral decomposition of the baryon masses following from this specific choice of the parameter set is given in Table 3.8. Such a decomposition serves as a rough check of the convergence pattern of the chiral expansion. The numerical values for the parameters in Tables 3.6,3.7 imply a smooth extrapolation of the baryon masses. The extrapolation of $M_{N}$ and $M_{\Omega}$ up to $m_{\pi}^{2}=0.48 \mathrm{GeV}^{2}$ and the lattice data of the BMW-group for three different values of the lattice spacing $a$ are shown in Figure 3.2. As described above, every step in the extrapolation includes a determination of the self consistent meson masses via

\footnotetext{
${ }^{6}$ One example is the combination of the unknown parameters $\stackrel{\circ}{M}[8]$ and $b_{0}$ in the expressions for the baryon mass shifts in (3.2). To disentangle them, a further observable, e.g. the nucleon sigma-term, is required. In the approach taken here, the determination of these parameters with the help of lattice data allows to predict the value of the nucleon sigma-term.
} 
the equation (3.10) and of the self consistent baryon masses via (3.2).

Having fixed the chiral parameters at the physical point and with the help of the lattice data for a single member of the flavour octet and decuplet, the baryon masses of all members of the multiplets are determined thereby. This is the consequence of the explicit $S U(3)$ flavour symmetry of the chiral Lagrangian and of the self consistency imposed on the baryon self energies in (3.2). A non-trivial and very stringent test of the $\chi \mathrm{PT}$-results follows from a comparison of the chiral extrapolation for all members of the flavour multiplets with the lattice data. The "global" plots containing the extrapolation for the flavour octet and decuplet baryons are given in Figures 3.3 and 3.4, respectively. The results of other lattice groups are also shown there. A quantitative comparison is not possible at this stage since the lattice data are not extrapolated into the continuum yet. The discrepancies among the various groups may serve as an estimate of the uncertainty.

The number of possible scenarios and the number of the free unknown parameters can be reduced by using the large- $N_{c}$ relations derived and discussed in Section 2.4. While the incorporation of the large- $N_{c}$ relations for the chiral symmetry conserving couplings is straightforward (Section 2.4.3), a direct application of the large- $N_{c}$ results for the symmetry breaking parameters (Section 2.4.1) is complicated by the non-analytical structure of the chiral loops and the running of the chiral parameters on the ultraviolet and on the infrared scales. The relations derived in Section 2.4.1 are independent of any renormalisation scales and are valid for the strict expansion in the flavour symmetry breaking parameter $\varepsilon$ only (see (2.42) and the discussion in Section 2.4.1 for further details). Inclusion of the chiral loops generates a non-trivial and a non-analytical dependence on the quark-masses. The interplay of the large- $N_{c}$ sum rules and chiral loop effects was not completed in this work and remains a task for the near future.

Despite the partial incorporation of the large- $N_{c}$ constraints, the results for the chiral extrapolation performed in this section look very promising. The used scheme allows a simultaneous determination of the octet and decuplet baryon masses and their extrapolation to higher quark masses. A reasonable convergence pattern achieved at this order applying the $\chi \mathrm{MS}$ renormalisation scheme doesn't cause any significant discrepancies between the extrapolated and the lattice results. The very astonishing result of numerous lattice studies, that the baryon masses show an almost linear quark mass dependence over a wide range of quark masses, can be reproduced by the very complicated non-linear quark mass dependence in $(3.3,3.4)$, once a well convergent expansion is achieved and the self consistence is imposed on the baryon masses. This all motivates a further more detailed and complete study of the large- $N_{c}$ constraints for the baryon masses at the one-loop level. 


\begin{tabular}{|c|c|c|c|c|c|}
\hline \multicolumn{2}{|c|}{$Q^{2}\left[\mathrm{GeV}^{-1}\right]$} & \multicolumn{2}{|c|}{$Q^{3}\left[\mathrm{GeV}^{-2}\right]$} & \multicolumn{2}{|c|}{$Q^{4}\left[\mathrm{GeV}^{-3}\right]$} \\
\hline $\bar{b}_{0}=-0.45$ & $\bar{d}_{0}=-0.17$ & $\bar{\zeta}_{0}=2.25$ & $\bar{\xi}_{0}=0.38$ & $\bar{c}_{0}^{\chi}=-0.86$ & $\bar{e}_{0}^{\chi}=-0.06$ \\
\hline $\bar{b}_{D}=0.10$ & $\bar{d}_{D}=-0.16$ & $\bar{\zeta}_{D}=-0.09$ & $\bar{\xi}_{D}=1.09$ & $\bar{c}_{1}^{\chi}=0.50$ & $\bar{e}_{1}^{\chi}=0.47$ \\
\hline $\bar{b}_{F}=-0.15$ & & $\bar{\zeta}_{F}=1.26$ & & $\bar{c}_{2}^{\chi}=0.30$ & $\bar{e}_{2}^{\chi}=0.13$ \\
\hline & & & & $\bar{c}_{3}^{\chi}=-0.31$ & $\bar{e}_{3}^{\chi}=-1.43$ \\
\hline & & & & $\bar{c}_{4}^{\chi}=-0.54$ & $\bar{e}_{4}^{\chi}=0.50$ \\
\hline & & & & $\bar{c}_{5}^{\chi}=2.88$ & \\
\hline & & & & $\bar{c}_{6}^{\chi}=0.89$ & \\
\hline
\end{tabular}

Table 3.6.: The chiral symmetry breaking parameters.

\begin{tabular}{|c|c|c|c|}
\hline \multicolumn{2}{|c|}{$Q^{0}[\mathrm{GeV}]$} & \multicolumn{2}{|c|}{$Q^{2}\left[\mathrm{GeV}^{-1}\right]$} \\
\hline$\stackrel{\circ}{M}_{[8]}=0.9$ & $\stackrel{\circ}{M}_{[10]}=1.2$ & $\begin{array}{l}g_{0}^{(S)}=-7.1 \\
g_{1}^{(S)}=-9.6 \\
g_{D}^{(S)}=5.6 \\
g_{F}^{(S)}=-0.9\end{array}$ & $\begin{array}{l}\tilde{h}_{1}^{(S)}=-0.004 \\
\tilde{h}_{2}^{(S)}=2.4 \\
\tilde{h}_{3}^{(S)}=-6.6\end{array}$ \\
\hline
\end{tabular}

Table 3.7.: The chiral symmetry conserving parameters. The corresponding $Q^{2}$ vectorcouplings are set to zero. As can be seen directly in $(3.3,3.4)$, this amounts to a finite renormalisation of the scalar couplings and to the omission of higher order terms. 


\begin{tabular}{|c|c|}
\hline$M_{N}[\mathrm{MeV}]$ & $900+225-106-80=939$ \\
$M_{\Lambda}[\mathrm{MeV}]$ & $900+334-81-37=1193$ \\
$M_{\Sigma}[\mathrm{MeV}]$ & $900+455-247+86=1193$ \\
$M_{\Xi}[\mathrm{MeV}]$ & $900+503-177+91=1193$ \\
\hline \hline$M_{\Delta}[\mathrm{MeV}]$ & $1156+185-39-69=1233$ \\
$M_{\Sigma}[\mathrm{MeV}]$ & $1156+233+17-21=1385$ \\
$M_{\Xi}[\mathrm{MeV}]$ & $1156+281+78+17=1532$ \\
$M_{\Omega}[\mathrm{MeV}]$ & $1156+330+134+51=1671$ \\
\hline
\end{tabular}

Table 3.8.: The decomposition of the baryon masses into their chiral momenta corresponding to the parameter set in Tables 3.6, 3.7. The 1st column contains the values of the baryon masses in the chiral limit. In the 2 nd column the values of the $Q^{2}$ tree-level contributions are given. The contributions of the $Q^{3}$ loop corrections follow in the 3rd column. Finaly, the tadpole- and the tree-level contribution of order $\mathcal{O}\left(Q^{4}\right)$ are cumulatively given in the last column. 


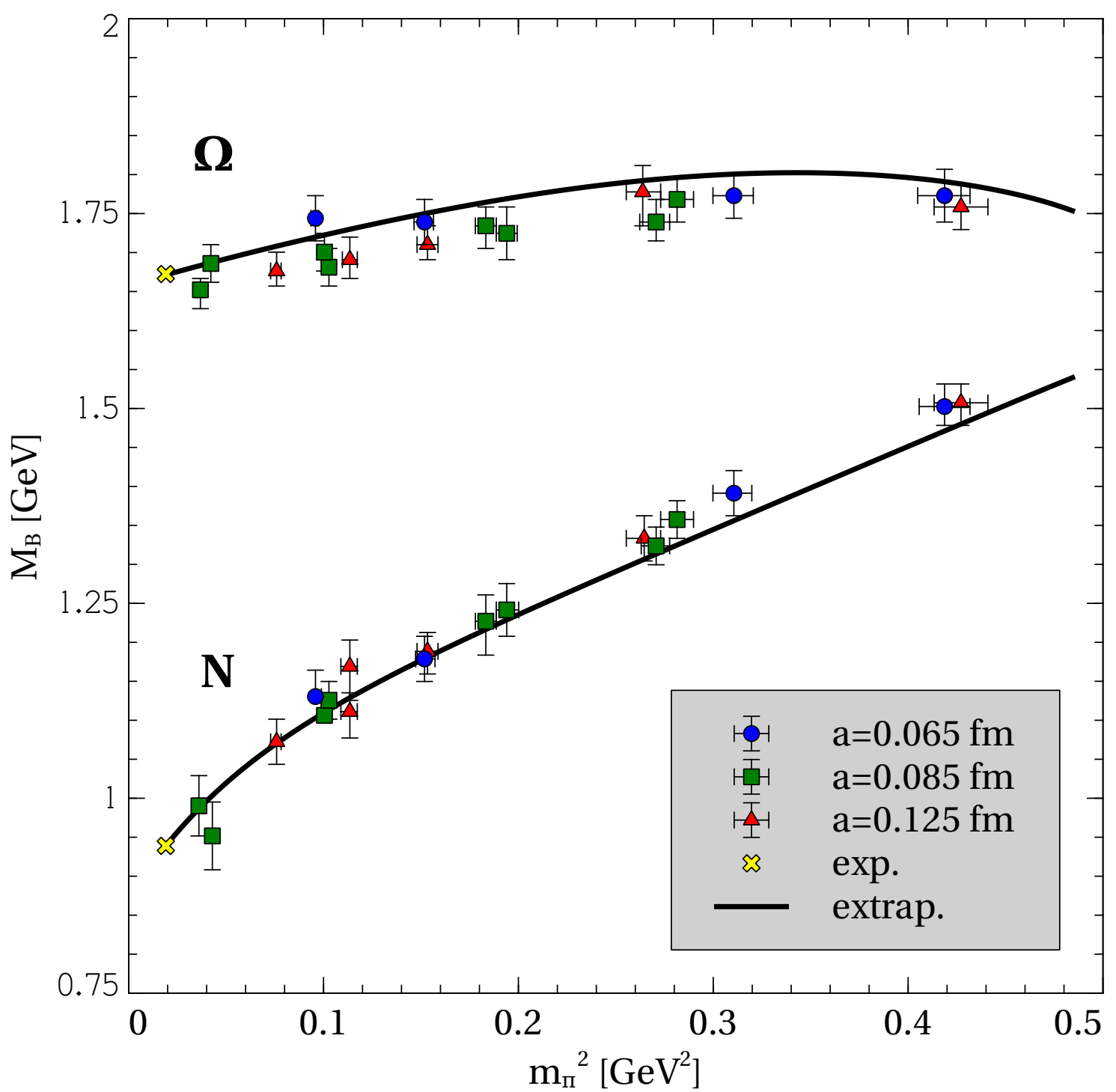

Figure 3.2.: Chiral extrapolation of the nucleon and omega masses. Lattice data are taken from [27]. 


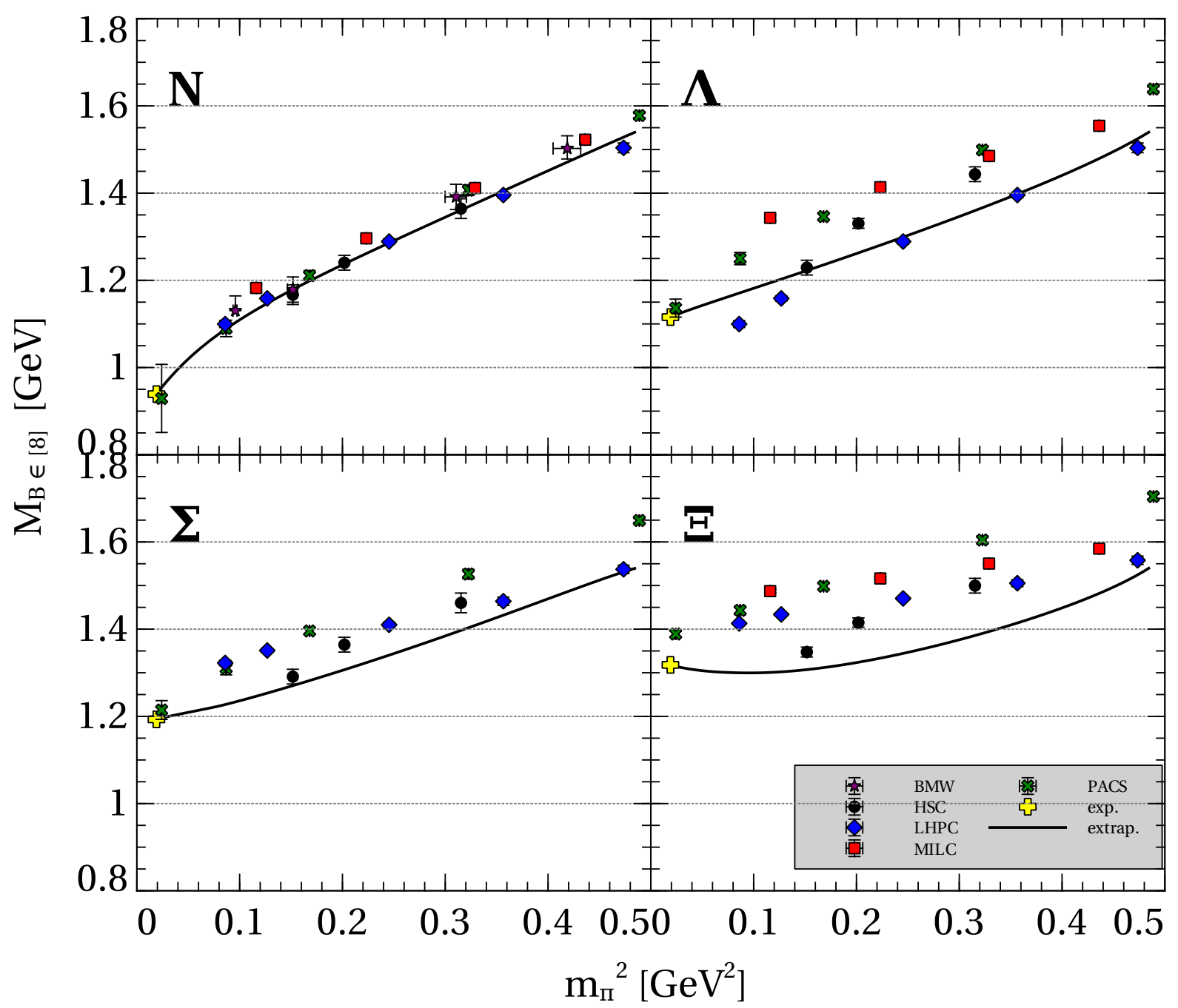

Figure 3.3.: Extrapolation of the octet baryon masses. Lattice data are taken from [27, $62,88,4,1]$. 


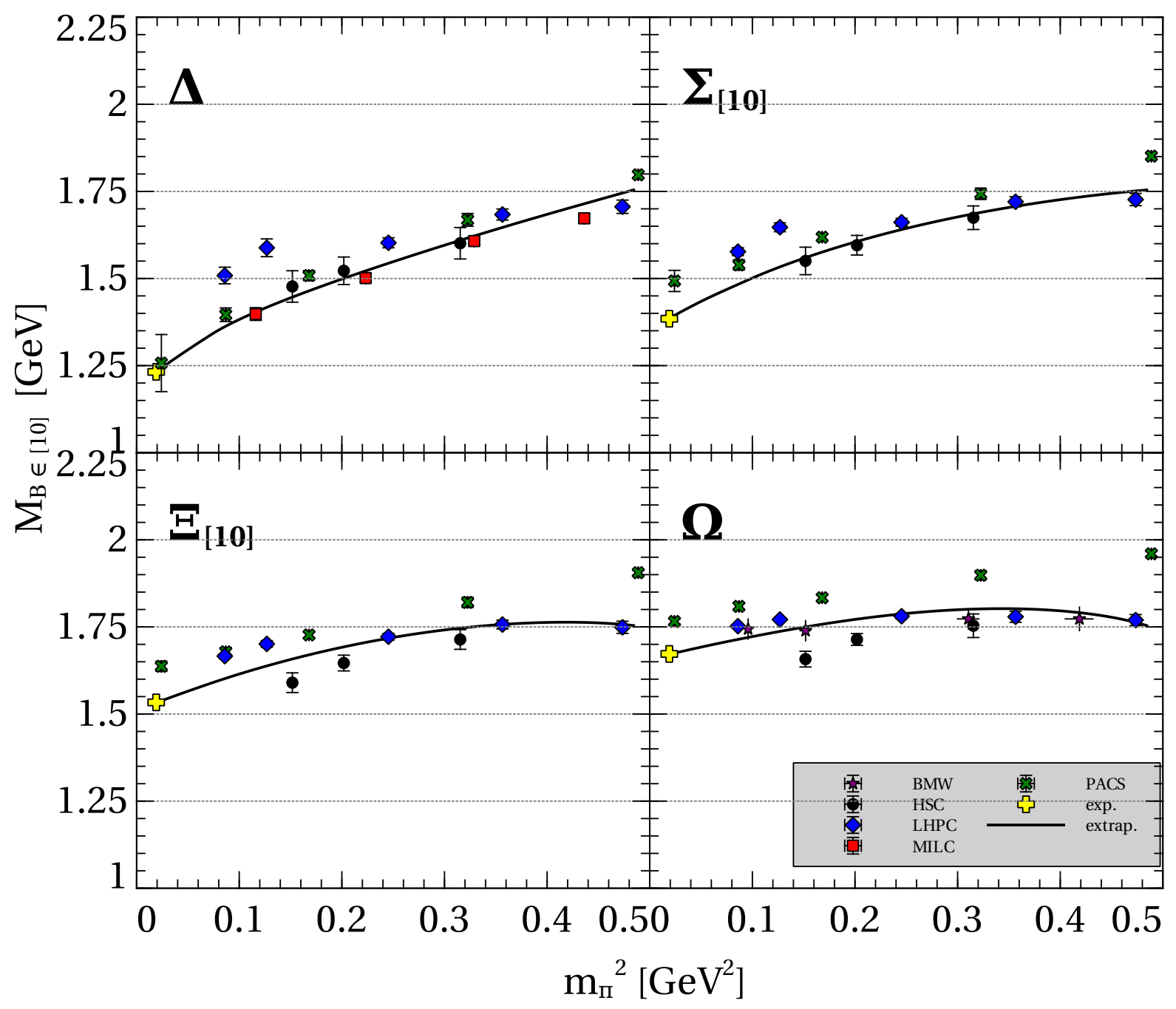

Figure 3.4.: Extrapolation of the decuplet baryon masses. Lattice data are taken from $[27,62,88,4,1]$. 


\section{Conclusions}

In the present work we have studied the interplay of three different approaches to Quantum Chromodynamics (QCD) in application to properties of the baryon ground states. We considered Chiral Perturbation Theory, $\chi \mathrm{PT}$, lattice QCD and large- $N_{c}$ QCD.

The effective field theory of QCD, Chiral Perturbation Theory, $\chi \mathrm{PT}$, is able to describe a plethora of phenomena in hadron physics at low energy. The most convincing results of this formulation are given in the two-flavour meson sector. The rigorous effective field theory formulation of QCD in this sector serves as a precision tool for QCD itself. The explicit inclusion of the strange quark worsens the predictive power in terms of a perturbative description of hadron properties. Though there are many experimental indications, that the approximate flavour symmetry $S U(3)$ of strongly interacting particles is indeed realised in nature, it was not clear for a long time, how to recover the approximate flavour symmetry in the results of Chiral Perturbation Theory. This problem is especially seen in the three-flavour baryon sector and is closely connected to the problem of the poor convergence properties of $\chi \mathrm{PT}$, once the strange quark is included explicitly. Many years after the formulation of $\chi \mathrm{PT}$ it was realised that a suitable renormalisation together with a partial summation of higher order terms can drastically improve the theory in the three-flavour baryon sector.

The formulation of QCD on discretised space-time, lattice QCD, is currently making great progress. Though the ultimate goal of lattice QCD is the calculation of the physical observables for the physical quark masses, the results of such calculations carried out at the quark masses set to higher values are still of importance. Such artificial high quark masses greatly reduce the run-time costs of lattice simulations and provide at the same time a method to determine the unknown coupling constants of the chiral Lagrangian. The inclusion of the explicit chiral symmetry breaking effects in the chiral Lagrangian provides the quark-mass dependence of hadron properties. Given such an explicit dependence on the quark masses calculated in the framework of the effective field theory at the physical point, the comparison with the results on the lattice at higher and unphysical quark masses is readily achieved.

In this work the $\chi \mathrm{MS}$ renormalisation scheme was applied to the all members of the flavour $S U(3)$ baryon octet and decuplet. A partial summation implied by a self consistency condition was developed and applied. We obtained a very good description of the empirical masses with a reasonable convergence pattern at sub-sub-leading order. The most remarkable result of this study is the almost linear quark mass dependence at large quark masses for many members of these multiplets. Such a linear dependence has been 
seen in lattice simulation over a wide range of the quark masses. This has been difficult to understand in application of the chiral Lagrangian. In our scheme it is the result of the particular summation scheme that defines a non-trivial and complicated dependence on the quark masses. In our study, the chiral parameters were constrained by the lattice data of the BMW-collaboration for the nucleon and for the omega baryon. This collaboration has carried out a very detailed and complete study of the hadron spectrum in unquenched simulations. Having constrained the parameters of the chiral Lagrangian by a single member of each flavour multiplet, a reasonable and simultaneous extrapolation for all members of the octet and decuplet was achieved.

The inclusion of higher order terms in the effective expansion is inevitably accompanied by the inclusion of new coupling constants, which in many cases can hardly be determined from experimental data. The description of QCD in the limit of an infinite number of colours provides another parametrisation of the physics of strongly interacting particles. By matching the results of this parametrisation to the low-energy description in $\chi \mathrm{PT}$, several relations among the coupling constants of the chiral Lagrangian can be established. This reduces the number of unknown parameters significantly. The success of the methods of QCD in the large- $N_{c}$ limit, if applied to the static properties of hadrons, motivated our further studies. In this work, several novel large- $N_{c}$ sum rules for the coupling constants relevant in the computation of the baryon masses at sub-sub-leading order were worked out. The chiral extrapolation discussed above was partially supplemented by these constraints.

It remains to study in more details the interrelationship of the large- $N_{c}$ expansion and the chiral expansion. So far the large- $N_{c}$ sum rules have been worked out at tree-level. A proper generalisation to the one-loop level is required. 


\section{Appendix A.}

\section{One-loop corrections of the baryon masses}

In this appendix we discuss several topics concerning the $Q^{3}$ and $Q^{4}$ one-loop corrections of the baryon masses used in Section 3.1.

\section{A.1. $Q^{4}$-contributions to the baryon self-energy}

Tadpole diagrams, evaluated with the relevant parts of the $Q^{2}$-interaction given in Section 1.4.2, give rise to the $Q^{4}$-mass corrections of baryons. Contributions to the self-energy of the octet baryons and to the self-energy tensor of the decuplet baryons are

$$
-i \Sigma_{B \in[8]}^{\text {tadpole }}(p)=-\frac{i}{(2 f)^{2}} \sum_{Q \in[8]} \int \frac{d^{d} k}{(2 \pi)^{d}} \frac{i \mu_{U V}^{4-d}}{k^{2}-m_{Q}^{2}+i \epsilon}\left(G_{B Q}^{(\chi, 2)}-G_{B Q}^{(S)} k^{2}-G_{B Q}^{(V)} \not k(k \cdot p)\right) .
$$

and

$$
\begin{aligned}
i \Sigma_{B \in[10]}^{\mu \nu \text { tadpole }}(p)=\frac{i}{(2 f)^{2}} & \sum_{Q \in[8]} \int \frac{d^{d} k}{(2 \pi)^{d}} \frac{i \mu_{U V}^{4-d}}{k^{2}-m_{Q}^{2}+i \epsilon} \\
& \times\left(G_{B Q}^{(\chi, 2)} g^{\mu \nu}-G_{B Q}^{I,(S)} g^{\mu \nu} k^{2}-G_{B Q}^{I I,(S)} k^{\mu} k^{\nu}-G_{B Q}^{(V)} g^{\mu \nu} \not k(k \cdot p)\right) .
\end{aligned}
$$

respectively. Here, the summation is done over all mesons propagating in the loop and the corresponding coefficients $G_{B Q}^{(\chi, 2)}, G_{B Q}^{(S)}$ and $G_{B Q}^{(V)}$ are collected in Table 3.4.

Dimensionaly regularised tensor integrals in (A.1) and (A.2) are reduced to the scalar tadpole integral

$$
I_{Q}=\int \frac{d^{d} k}{(2 \pi)^{d}} \mu_{U V}^{4-d} \frac{i}{k^{2}-m_{Q}^{2}+i \epsilon}
$$


via

$$
\begin{gathered}
i \int \frac{d^{d} k}{(2 \pi)^{d}} \mu_{U V}^{4-d} \frac{k^{2}}{k^{2}-m_{Q}^{2}+i \epsilon}=m_{Q}^{2} I_{Q}, \quad i \int \frac{d^{d} k}{(2 \pi)^{d}} \mu_{U V}^{4-d} \frac{\not k(k \cdot p)}{k^{2}-m_{Q}^{2}+i \epsilon}=\not p \frac{m_{Q}^{2}}{d} I_{Q} \\
i \int \frac{d^{d} k}{(2 \pi)^{d}} \mu^{4-d} \frac{k^{\mu} k^{\nu}}{k^{2}-m_{Q}^{2}+i \epsilon}=g^{\mu \nu} \frac{m_{Q}^{2}}{d} I_{Q} .
\end{gathered}
$$

Having this, one gets for $\Sigma_{B \in[8]}^{\text {tadpole }}(p)$

$$
\Sigma_{B \in[8]}^{\text {tadpole }}(p)=\frac{1}{(2 f)^{2}} \sum_{Q \in[8]}\left(G_{B Q}^{(\chi, 2)}-G_{B Q}^{(S)} m_{Q}^{2}-G_{B Q}^{(V)} \not p \frac{m_{Q}^{2}}{d}\right) I_{Q},
$$

and for $\Sigma_{B \in[10]}^{\mu \nu \text { tadpole }}(p)$

$$
\begin{aligned}
\Sigma_{B \in[10]}^{\mu \nu \text { tadpole }}(p) & =\frac{1}{(2 f)^{2}} g^{\mu \nu} \sum_{Q \in[8]}\left(G_{B Q}^{(\chi, 2)}-G_{B Q}^{I,(S)} m_{Q}^{2}-G_{B Q}^{I I,(S)} \frac{m_{Q}^{2}}{d}-G_{B Q}^{(V)} \frac{m_{Q}^{2}}{d} \not p\right) I_{Q} \\
& =\frac{1}{(2 f)^{2}} g^{\mu \nu} \sum_{Q \in[8]}\left(G_{B Q}^{(\chi, 2)}-G_{B Q}^{(S)} m_{Q}^{2}-G_{B Q}^{(V)} \frac{m_{Q}^{2}}{d} \not p\right) I_{Q},
\end{aligned}
$$

with the abbreviation, used in the last line:

$$
G_{B Q}^{(S)} \equiv G_{B Q}^{I,(S)}+\frac{1}{d} G_{B Q}^{I I,(S)} \quad \text { for } B \in[10] .
$$

Common problem for all fields with spin $s \geqq 1$ are contributions to the self-energy from non-leading spin components. These contributions result in non-pole terms in the selfenergy tensors and are not relevant for the calculation of mass corrections. By means of an appropriate complete set of projectors (see e.g. $[65,79]$ ), the spin-3/2 propagator $S^{\mu \nu}$ for decuplet baryons can be decomposed as in

$$
S^{\mu \nu}(p)=\frac{-1}{\sqrt{p^{2}}-\stackrel{\circ}{M}_{[10]}-\Sigma(p)} P_{\frac{3}{2}}^{\mu \nu}(p)+(\text { spin-1/2 contributions }),
$$

with the explicit representation of the spin-3/2 projector in $d$ dimensions

$$
\begin{gathered}
P_{3 / 2}^{\mu \nu}(p)=\left(g^{\mu \nu}-\frac{p^{\mu} p^{\nu}}{p^{2}}\right) P_{+}(p)-V^{\mu}(p) P_{-}(p) V^{\nu}(p), \\
P_{ \pm}=\frac{1}{2}\left(1 \pm \frac{\not p}{\sqrt{p^{2}}}\right), \quad V^{\mu}(p)=\frac{1}{\sqrt{d-1}}\left(\gamma^{\mu}-\frac{\not p p^{\mu}}{p^{2}}\right) .
\end{gathered}
$$

Having stated this and the relation $\operatorname{tr}\left(P_{3 / 2}^{\mu \nu}(p) g_{\mu \nu}\right)=\frac{d(d-2)}{2}$, projection onto the spin-3/2 part of $\Sigma_{B \in[10]}^{\mu \nu \text { tadpole }}$ yields finally

$$
\begin{aligned}
\Sigma_{B \in[10]}^{\text {tadpole }}(p) & =\frac{2}{d(d-2)} \operatorname{tr}\left(P_{3 / 2}^{\mu \nu}(p) \Sigma_{B \in[10] \mu \nu}^{\text {tadpole }}(p)\right) \\
& =\frac{1}{(2 f)^{2}} \sum_{Q \in[8]}\left(G_{B Q}^{(\chi, 2)}-G_{B Q}^{(S)} m_{Q}^{2}-G_{B Q}^{(V)} \frac{m_{Q}^{2}}{d} \not p\right) I_{Q}
\end{aligned}
$$




\section{A.2. Scale dependence of the chiral parameters}

The running of the chiral couplings on the infrared and ultraviolet scales was discussed in Section 3.1 in detail. The explicit expressions the coefficients $\Gamma^{I R}$ and $\Gamma^{U V}$, as definied in (3.7), for the $Q^{2}$ and $Q^{3}$ couplings were stated in Table 3.1. Here, these coefficient are specified for the $Q^{4}$ parameters:

$$
\begin{aligned}
& \Gamma_{c_{0}}^{\mathrm{UV}}=\frac{2}{27}\left(9\left(D^{2}-15 F^{2}\right) b_{D}-18 D F b_{F}+11 C^{2} d_{D}\right)+\frac{1}{3}\left(20 b_{0}+12 b_{D}\right) \\
& -\frac{1}{36}\left(30 g_{0}^{(S)}+9 g_{1}^{(S)}+26 g_{D}^{(S)}\right)-\frac{1}{144}\left(30 g_{0}^{(V)}+9 g_{1}^{(V)}+26 g_{D}^{(V)}\right) \stackrel{\circ}{M}[8] \\
& +\frac{5}{432}\left(96\left(D^{2}+3 F^{2}\right)+55 C^{2}\right) \stackrel{\circ}{M}[8] \text {, } \\
& \Gamma_{c_{1}}^{\mathrm{UV}}=-\frac{4}{27}\left(3\left(13 D^{2}+9 F^{2}\right) b_{D}-90 D F b_{F}-5 C^{2} d_{D}\right) \\
& -\frac{1}{6} g_{1}^{(S)}-\frac{1}{24} g_{1}^{(V)} \stackrel{\circ}{M}[8] \\
& +\frac{1}{216}\left(96\left(D^{2}-3 F^{2}\right)-25 C^{2}\right) \stackrel{\circ}{M_{[8]}^{-1}} \text {, } \\
& \Gamma_{c_{2}}^{\mathrm{UV}}=\frac{4}{9}\left(3\left(D^{2}+9 F^{2}\right) b_{D}-18 D F b_{F}-4 C^{2} d_{D}\right) \\
& +\frac{2}{3} b_{D}+\frac{1}{4}\left(g_{1}^{(S)}+g_{D}^{(S)}\right)+\frac{1}{16}\left(g_{1}^{(V)}+g_{D}^{(V)}\right) \stackrel{\circ}{M}_{[8]} \\
& +\frac{1}{36}\left(12\left(D^{2}-3 F^{2}\right)+25 C^{2}\right) \stackrel{\circ}{M_{[8]}^{-1}} \text {, } \\
& \Gamma_{c_{3}}^{\mathrm{UV}}=\frac{4}{9}\left(12 D^{2} b_{F}+C^{2} d_{D}\right)+\frac{2}{3} b_{F}+\frac{1}{4} g_{F}^{(S)}+\frac{1}{16} g_{F}^{(V)} \stackrel{\circ}{M}_{[8]} \\
& -\frac{1}{48}\left(96 D F+25 C^{2}\right) \stackrel{\circ}{M_{[8]}^{-1}} \text {, } \\
& \Gamma_{c_{4}}^{\mathrm{UV}}=\frac{2}{27}\left(3 D^{2}\left(9 b_{0}+8 b_{D}\right)-27 F^{2}\left(3 b_{0}+8 b_{D}\right)+180 D F b_{F}+2 C^{2}\left(9 d_{0}+8 d_{D}\right)\right) \\
& +\frac{44}{9} b_{D}-\frac{1}{18}\left(9 g_{1}^{(S)}+13 g_{D}^{(S)}\right)-\frac{1}{72}\left(9 g_{1}^{(V)}+13 g_{D}^{(V)}\right) \stackrel{\circ}{M}_{[8]} \\
& -\frac{2}{27}\left(21\left(D^{2}-3 F^{2}\right)+25 C^{2}\right) \stackrel{\circ}{M}[8] \text {, } \\
& \Gamma_{c_{5}}^{\mathrm{UV}}=-\frac{2}{27}\left(6\left(13 D^{2}+9 F^{2}\right) b_{F}+90 D F b_{0}+C^{2}\left(15 d_{0}+19 d_{D}\right)\right) \\
& +\frac{44}{9} b_{F}-\frac{13}{18} g_{F}^{(S)}-\frac{13}{72} g_{F}^{(V)} \stackrel{\circ}{M}[8] \\
& +\frac{13}{216}\left(96 D F+25 C^{2}\right) \stackrel{\circ}{M}[8] \text {, } \\
& \Gamma_{c_{6}}^{\mathrm{UV}}=-\frac{2}{27}\left(3 D^{2}\left(26 b_{0}+21 b_{D}\right)+3 F^{2}\left(18 b_{0}-27 b_{D}\right)+90 D F b_{F}+C^{2}\left(42 d_{0}+19 d_{D}\right)\right) \\
& +\frac{44}{9} b_{0}-\frac{1}{108}\left(66 g_{0}^{(S)}-27 g_{1}^{(S)}-8 g_{D}^{(S)}\right)-\frac{1}{432}\left(66 g_{0}^{(V)}-27 g_{1}^{(V)}-8 g_{D}^{(V)}\right) \stackrel{\circ}{M}_{[8]} \\
& +\frac{1}{432}\left(896 D^{2}-576 F^{2}+875 C^{2}\right) \stackrel{\circ}{M_{[8]}^{-1}} \text {, } \\
& \Gamma_{e_{0}}^{\mathrm{UV}}=\frac{2}{81}\left(27 C^{2}\left(b_{D}-b_{F}\right)-5 H^{2} d_{D}\right)+\frac{1}{3}\left(20 d_{0}+6 d_{D}\right)
\end{aligned}
$$




$$
\begin{aligned}
& -\frac{1}{18}\left(15 \tilde{h}_{1}^{(S)}+13 \tilde{h}_{2}^{(S)}+9 \tilde{h}_{3}^{(S)}\right)-\frac{1}{72}\left(15 h_{1}^{(V)}+13 h_{2}^{(V)}+9 h_{3}^{(V)}\right) \stackrel{\circ}{M_{[8]}} \\
& +\frac{1}{432} \stackrel{\circ}{M}_{[8]}^{-1}\left(81 C^{2}+196 H^{2}\right) \\
\Gamma_{e_{1}}^{\mathrm{UV}} & =\frac{4}{27}\left(9 C^{2} b_{F}+5 H^{2} d_{D}\right)-\frac{1}{3} \tilde{h}_{3}^{(S)}-\frac{1}{12} h_{3}^{(V)} \stackrel{\circ}{M}[8] \\
& +\frac{1}{216} \stackrel{\circ}{M}_{[8]}^{-1}\left(27 C^{2}-56 H^{2}\right) \\
\Gamma_{e_{2}}^{\mathrm{UV}} & =-\frac{4}{27}\left(9 C^{2} b_{D}+5 H^{2} d_{D}\right) \\
& +\frac{2}{3} d_{D}+\frac{1}{2}\left(\tilde{h}_{2}^{(S)}+\tilde{h}_{3}^{(S)}\right)+\frac{1}{8}\left(h_{2}^{(V)}+h_{3}^{(V)}\right) \stackrel{\circ}{M_{[8]}} \\
& -\frac{1}{144} \stackrel{\circ}{M_{[8]}^{-1}}\left(27 C^{2}+28 H^{2}\right), \\
\Gamma_{e_{3}}^{\mathrm{UV}} & =-\frac{2}{81}\left(27 C^{2}\left(b_{0}+2 b_{F}\right)+5 H^{2}\left(9 d_{0}+5 d_{D}\right)\right) \\
& +\frac{44}{9} d_{D}-\frac{1}{9}\left(13 \tilde{h}_{2}^{(S)}+9 \tilde{h}_{3}^{(S)}\right)-\frac{1}{36}\left(13 h_{2}^{(V)}+9 h_{3}^{(V)}\right) \stackrel{\circ}{M}[8] \\
& +\frac{1}{216} \stackrel{\circ}{M_{[8]}^{-1}}\left(81 C^{2}+196 H^{2}\right), \\
\Gamma_{e_{4}}^{\mathrm{UV}} & =-\frac{2}{81}\left(27 C^{2}\left(b_{0}+b_{D}-b_{F}\right)+25 H^{2} d_{0}\right) \\
& +\frac{44}{9} d_{0}-\frac{1}{54}\left(33 \tilde{h}_{2}^{(S)}-4 \tilde{h}_{2}^{(S)}\right)-\frac{1}{216}\left(33 h_{1}^{(V)}-4 h_{2}^{(V)}\right) \stackrel{\circ}{M}[8] \\
& -\frac{7}{81} \stackrel{\circ}{M}[8] H^{2},
\end{aligned}
$$

and

$$
\begin{aligned}
\Gamma_{c_{0}}^{\mathrm{IR}}= & -\frac{64}{81}\left(45 D^{2}\left(b_{D}^{2}+3 b_{F}^{2}\right)+27 F^{2}\left(5 b_{D}^{2}+3 b_{F}^{2}\right)+324 D F b_{D} b_{F}+2 C^{2} d_{D}^{2}\right) \\
- & \frac{16}{27 f^{2}}\left(D^{2}\left(5 L_{5}-36 L_{7}-22 L_{8}\right)+18 F^{2}\left(5 L_{5}+12 L_{7}-6 L_{8}\right)\right. \\
& \left.\quad+C^{2}\left(11 L_{5}+96 L_{7}+54 L_{8}\right)\right)-\frac{11}{27} C^{2}\left(\stackrel{\circ}{M}_{[8]}^{-2}+8 d_{D} \stackrel{\circ}{M}_{[8]}^{-1}\right), \\
\Gamma_{c_{1}}^{\mathrm{IR}}= & -\frac{128}{81}\left(9\left(D^{2}-3 F^{3}\right)\left(b_{D}^{2}-3 b_{F}^{2}\right)-7 C^{2} d_{D}^{2}\right) \\
- & \frac{32}{27 f^{2}}\left(6 D^{2}-18 F^{2}-C^{2}\right)\left(L_{5}-12 L_{7}-6 L_{8}\right) \\
+ & \frac{2}{27} C^{2}\left(\stackrel{\circ}{M_{[8]}^{-2}}-40 d_{D} \stackrel{\circ}{M}_{[8]}^{-1}\right), \\
\Gamma_{c_{2}}^{\mathrm{IR}}= & -\frac{32}{27}\left(9\left(D^{2}-3 F^{2}\right)\left(b_{D}^{2}-3 b_{F}^{2}\right)-324 D F b_{D} b_{F}+11 C^{2} d_{D}^{2}\right) \\
- & \frac{16}{9 f^{2}}\left(3\left(D^{2}-3 F^{2}\right)\left(L_{5}+24 L_{7}+6 L_{8}\right)+4 C^{2}\left(L_{5}+6 L_{7}\right)\right) \\
& -\frac{4}{9} C^{2}\left(\stackrel{\circ}{M_{[8]}^{-2}}-16 d_{D} \stackrel{\circ}{M}_{[8]}^{-1}\right), \\
\Gamma_{c_{3}}^{\mathrm{IR}}= & -\frac{32}{3}\left(2\left(5 D^{2}+9 F^{2}\right) b_{D} b_{F}+6 D F\left(b_{D}^{2}-3 b_{F}^{2}\right)+C^{2} d_{D}^{2}\right) \\
+ & \frac{16}{9 f^{2}}\left(6 \operatorname{DF}^{2}+C^{2}\right)\left(3 L_{5}+24 L_{7}+2 L_{8}\right)
\end{aligned}
$$




$$
\begin{aligned}
& +\frac{1}{9} C^{2}\left(3 \stackrel{\stackrel{\circ}{M}_{[8]}^{-2}}{M^{2}}-16 d_{D} \stackrel{\circ}{M}_{[8]}^{-1}\right), \\
& \Gamma_{c_{4}}^{\mathrm{IR}}=\frac{64}{81}\left(9\left(D^{2}-3 F^{2}\right)\left(7 b_{D}^{2}-3 b_{F}^{2}+9 b_{0} b_{D}\right)+486 D F b_{0} b_{F}+C^{2}\left(2 d_{D}^{2}-27 d_{0} d_{D}\right)\right) \\
& +\frac{32}{27 f^{2}}\left(3\left(D^{2}-3 F^{2}\right)\left(9 L_{4}+7 L_{5}-6\left(3 L_{6}-4 L_{7}+L_{8}\right)\right)\right. \\
& \left.+2 C^{2}\left(9 L_{4}+2\left(4 L_{5}-9 L_{6}+6 L_{7}-6 L_{8}\right)\right)\right) \\
& +\frac{16}{27} C^{2}\left(2 \stackrel{\circ}{M_{[8]}^{-2}}-\left(9 d_{0}+8 d_{D}\right) \stackrel{\circ}{M_{[8]}^{-1}}\right) \text {, } \\
& \Gamma_{c_{5}}^{\mathrm{IR}}=-\frac{64}{27}\left(9\left(5 D^{2}+9 F^{2}\right) b_{0} b_{F}-6 D F\left(13 b_{D}^{2}-9 b_{F}^{2}+15 b_{0} b_{D}\right)+C^{2}\left(2 d_{D}^{2}+15 d_{0} d_{D}\right)\right) \\
& -\frac{32}{27 f^{2}}\left(6 D F+C^{2}\right)\left(15 L_{4}+13 L_{5}-6\left(5 L_{6}-4 L_{7}+3 L_{8}\right)\right) \\
& -\frac{2}{27} C^{2}\left(13 \stackrel{\circ}{M}_{[8]}^{-2}-4\left(15 d_{0}+19 d_{D}\right) \stackrel{\circ}{M}_{[8]}^{-1}\right) \text {, } \\
& \Gamma_{c_{6}}^{\mathrm{IR}}=-\frac{32}{27}\left(2 D^{2}\left(45 b_{0}^{2}+28 b_{D}^{2}-18 b_{F}^{2}+78 b_{0} b_{D}\right)+9 F^{2}\left(18 b_{0}^{2}-4 b_{D}^{2}+12 b_{0} b_{D}\right)\right. \\
& \left.+216 D F b_{0} b_{F}+3 C^{2}\left(15 d_{0}^{2}+d_{D}^{2}+6 d_{0} d_{D}\right)\right) \\
& -\frac{16}{27 f^{2}}\left(4 D^{2}\left(39 L_{4}+14 L_{5}-78 L_{6}+30 L_{7}-18 L_{8}\right)+36 F^{2}\left(3 L_{4}-L_{5}-6 L_{6}-6 L_{7}\right)\right. \\
& \left.+C^{2}\left(84 L_{4}+35 L_{5}-6\left(28 L_{6}-8 L_{7}+9 L_{8}\right)\right)\right) \\
& -\frac{1}{27} C^{2}\left(35 \stackrel{\circ}{M}_{[8]}^{-2}-8\left(42 d_{0}+19 d_{D}\right) \stackrel{\circ}{M}_{[8]}^{-1}\right) \text {, } \\
& \Gamma_{e_{0}}^{\mathrm{IR}}=-\frac{32}{243}\left(81 C^{2}\left(b_{D}^{2}+b_{F}^{2}-2 b_{D} b_{F}\right)+5 H^{2} d_{D}^{2}\right) \\
& -\frac{16}{81 f^{2}}\left(27 C^{2}+35 H^{2}\right)\left(L_{5}-2 L_{8}\right) \\
& -\frac{1}{81}\left(27 C^{2}+14 H^{2}\right) \stackrel{\circ}{M_{[8]}^{-2}}+\frac{8}{3} C^{2}\left(b_{D}-b_{F}\right) \stackrel{\circ}{M_{[8]}^{-1}}, \\
& \Gamma_{e_{1}}^{\mathrm{IR}}=-\frac{64}{243}\left(27 C^{2}\left(b_{D}^{2}-3 b_{F}^{2}\right)-20 H^{2} d_{D}^{2}\right) \\
& -\frac{32}{81 f^{2}}\left(9 C^{2}-10 H^{2}\right)\left(L_{5}-12 L_{7}-6 L_{8}\right) \\
& -\frac{2}{81}\left(9 C^{2}-4 H^{2}\right) \stackrel{\circ}{M_{[8]}^{-2}}+\frac{16}{3} C^{2} b_{F} \stackrel{\circ}{M}_{[8]}^{-1}, \\
& \Gamma_{e_{2}}^{\mathrm{IR}}=+\frac{32}{81}\left(27 C^{2}\left(b_{D}^{2}-3 b_{F}^{2}-6 b_{D} b_{F}\right)-35 H^{2} d_{D}^{2}\right) \\
& +\frac{16}{27 f^{2}}\left(9 C^{2}\left(L_{5}-2 L_{8}\right)+5 H^{2}\left(L_{5}+24 L_{7}+6 L_{8}\right)\right) \\
& +\frac{1}{27}\left(9 C^{2}+2 H^{2}\right) \stackrel{\circ}{M}[8]-\frac{16}{3} C^{2} b_{D} \stackrel{\circ}{M}_{[8]}^{-1}, \\
& \Gamma_{e_{3}}^{\mathrm{IR}}=-\frac{64}{243}\left(81 C^{2}\left(b_{D}^{2}-b_{F}^{2}+b_{0} b_{D}+3 b_{0} b_{F}\right)+H^{2}\left(135 d_{0}^{2}+10 d_{D}^{2}\right)\right) \\
& -\frac{32}{81 f^{2}}\left(27 C^{2}\left(L_{4}+L_{5}-2 L_{6}-2 L_{8}\right)+5 H^{2}\left(9 L_{4}+7 L_{5}-18 L_{6}+24 L_{7}-6 L_{8}\right)\right) \\
& -\frac{2}{81}\left(27 C^{2}+14 H^{2}\right) \stackrel{\circ}{M_{[8]}^{-2}}-\frac{8}{3} C^{2}\left(b_{0}+2 b_{F}\right) \stackrel{\circ}{M_{[8]}^{-1}} \text {, }
\end{aligned}
$$




$$
\begin{aligned}
\Gamma_{e_{4}}^{\mathrm{IR}} & =-\frac{64}{81}\left(27 C^{2}\left(b_{0}^{2}+b_{0} b_{D}-b_{0} b_{F}\right)+5 H^{2}\left(6 d_{0}^{2}+d_{0} d_{D}\right)\right) \\
& -\frac{32}{243 f^{2}}\left(81 C^{2}\left(L_{4}-2 L_{6}\right)+5 H^{2}\left(15 L_{4}-2 L_{5}-30 L_{6}-12 L_{7}\right)\right) \\
& +\frac{8}{243} H^{2} \stackrel{\circ}{M}[8]-\frac{8}{3} C^{2}\left(b_{0}+b_{D}-b_{F}\right) \stackrel{\circ}{M}_{[8]}^{-1} .
\end{aligned}
$$

\section{A.3. Finite terms}

In the framework of the dimensional regularisation, ultraviolet divergencies manifest themselves as pole terms in the scalar intergrals for $d=4$. In the case of $d$-dependent factors in front of the divergent integrals, the limiting process $d \rightarrow 4$ leads to finite terms. The origin of such $d$-dependent factors is twofold. Firstly, such factors naturaly arise in the process of the Passarino-Veltman reduction applied to the tensor integrals. The second reason for the occurence of the $d$-dependent factors is the explicit $d$-dependence of the spin-3/2 propagators.

For the case of interest in this section, only the scalar mesonic integral $I_{Q}$ needs to be considered. E.g., for the one of the simplest case of the $d$-dependent factors in front of $I_{Q}$, it holds

$$
\frac{1}{d} I_{Q}=\left(\frac{1}{4}-\frac{d-4}{16}+\mathcal{O}\left((d-4)^{2}\right)\right) I_{Q}=\frac{1}{4} I_{Q}-\frac{1}{(4 \pi)^{2}} \frac{m_{Q}^{2}}{8}+\mathcal{O}(d-4) .
$$

Such finite terms, expanded in powers of the quark masses, can be absorbed by a finite number of the symmetry breaking counter terms and are not kept explicitly in the perturbative chiral expansion. This absorbtion, which amounts to a finite renormalisation of the symmetry breaking couplings, is demonstrated in this section for the $Q^{3}$ and $Q^{4}$ one-loop corrections to the baryon self-energy.

Using the explicit expressions for the $Q^{3}$ and $Q^{4}$ one-loop corrections in $d$ dimensions, stated in [79] and Appendix A.1, respectively, the finite terms are easily worked out. It holds for the finite part of the octet baryon self-energy:

$$
\begin{aligned}
\sum_{B \in[8]}^{\mathrm{fin} .}(p)= & \sum_{Q \in[8], R \in[10]}\left(\frac{G_{Q R}^{(B)}}{2 f}\right)^{2}\left[\frac { 1 } { 6 M _ { R } } \frac { 1 } { 4 8 \pi ^ { 2 } } \left(3 m_{Q}^{4}+\left(M_{R}^{2}-p^{2}\right) m_{Q}^{2}\right.\right. \\
& \left.\quad+\frac{m_{Q}^{2}}{M_{R}^{2}-m_{Q}^{2}}\left(-m_{Q}^{4}+2\left(M_{R}^{2}+p^{2}\right) m_{Q}^{2}-\left(M_{R}^{2}-p^{2}\right)^{2}\right)\right) \\
+ & \frac{1}{12 M_{R}^{2} p^{2}} \frac{1}{96 \pi^{2}}\left(2 m_{Q}^{6}+7 p^{2} m_{Q}^{4}-4 M_{R}^{2} m_{Q}^{4}+2\left(M_{R}^{4}-p^{4}\right) m_{Q}^{2}\right. \\
& +\frac{2 m_{Q}^{2}}{M_{R}^{2}-m_{Q}^{2}}\left(m_{Q}^{6}-3\left(M_{R}^{2}+p^{2}\right) m_{Q}^{4}+\left(3 p^{4}+3 M_{R}^{4}+2 M_{R}^{2} p^{2}\right) m_{Q}^{2}\right.
\end{aligned}
$$




$$
\begin{gathered}
\left.\left.\left.-\left(M_{R}^{2}-p^{2}\right)^{2}\left(M_{R}^{2}+p^{2}\right)\right)\right)\right] \\
-\frac{1}{(2 f)^{2}} \frac{1}{(4 \pi)^{2}} \frac{1}{8} \sum_{Q \in[8]} G_{B Q}^{(V)} \not m_{Q}^{4},
\end{gathered}
$$

and for the finite part of the decuplet baryon self-energy:

$$
\begin{aligned}
\Sigma_{B \in[10]}^{\text {fin. }}(p)=- & \sum_{Q \in[8], R \in[8]}\left(\frac{G_{Q R}^{(B)}}{2 f}\right)^{2}\left[\frac { M _ { R } } { 1 2 p ^ { 2 } } \frac { 1 } { 2 4 \pi ^ { 2 } } \left(\left(-m_{Q}^{2}+M_{R}^{2}-p^{2}\right) m_{Q}^{2}\right.\right. \\
& \left.+\frac{m_{Q}^{2}}{M_{R}^{2}-m_{Q}^{2}}\left(-m_{Q}^{4}+2\left(M_{R}^{2}+p^{2}\right) m_{Q}^{2}-\left(M_{R}^{2}-p^{2}\right)^{2}\right)\right) \\
+ & \sqrt{p^{2}} \frac{1}{24 p^{4}} \frac{1}{24 \pi^{2}}\left(\left(m_{Q}^{4}-2\left(M_{R}^{2}+2 p^{2}\right) m_{Q}^{2}+M_{R}^{4}-p^{4}\right) m_{Q}^{2}+\frac{7 p^{2} m_{Q}^{4}}{4}\right. \\
& +\frac{m_{Q}^{2}}{M_{R}^{2}-m_{Q}^{2}}\left(m_{Q}^{6}-3\left(M_{R}^{2}+p^{2}\right) m_{Q}^{4}+\left(3 M_{R}^{4}+2 p^{2} M_{R}^{2}+3 p^{4}\right) m_{Q}^{2}\right. \\
- & \left.\left.\left.\left(M_{R}^{2}-p^{2}\right)^{2}\left(M_{R}^{2}+p^{2}\right)\right)\right)\right] \\
+ & \sum_{Q \in[8], R \in[10]}\left(\frac{G_{Q R}^{(B)}}{2 f}\right)^{2}\left[\frac { 1 } { 1 8 M _ { R } p ^ { 2 } } \frac { 1 } { 9 6 \pi ^ { 2 } } \frac { p ^ { 2 } } { M _ { R } ^ { 2 } - m _ { Q } ^ { 2 } } \left(-15 m_{Q}^{6}\right.\right. \\
+ & +\sqrt{p^{2}} \frac{1}{36 M_{R}^{2} p^{4}} \frac{1}{96 \pi^{2}} \frac{p^{2}}{M_{R}^{2}-m_{Q}^{2}}\left(3\left(M_{R}^{2}+22 p^{2}\right) m_{Q}^{4}+8\left(M_{R}^{4}-p^{4}\right) m_{Q}^{2}\right) \\
& \quad \frac{-\left(M_{R}^{4}-105 m_{R}^{2} m^{2}+8 p^{4}\right) m_{Q}^{4}}{(2 f)^{2}} \frac{1}{(4 \pi)^{2}} \frac{1}{8} \sum_{Q \in[8]}\left(G_{B Q}^{(I I, S)}+G_{B Q}^{(V)} \not\right) m_{Q}^{4} .
\end{aligned}
$$

The finite renormalisations of the $Q^{2} Q^{3}$ couplings by these expressions was summarised in Table 3.1. Upon working out the next chiral order in the above expressions, the finite renormalisation of the $Q^{4}$ octet couplings read:

$$
\begin{aligned}
& \Gamma_{c_{0}}^{\text {fin. }}=-\frac{1}{81} C^{2}\left(\frac{517}{32} \stackrel{\circ}{M}_{[8]}^{-1}+22 d_{D}\right)-\frac{1}{288}\left(30 g_{0}^{(V)}+9 g_{1}^{(V)}+26 g_{D}^{(V)}\right) \stackrel{\circ}{M}[8], \\
& \Gamma_{c_{1}}^{\text {fin. }}=\frac{1}{81} C^{2}\left(\frac{47}{16} \stackrel{\circ}{M_{[8]}^{-1}}-20 d_{D}\right)-\frac{1}{48} g_{1}^{(V)} M_{[8]}, \\
& \Gamma_{c_{2}}^{\text {fin. }}=-\frac{1}{27} C^{2}\left(\frac{47}{8} \stackrel{\circ}{M_{[8]}^{-1}}-16 d_{D}\right)+\frac{1}{32}\left(g_{1}^{(V)}+g_{D}^{(V)}\right) \stackrel{\circ}{M}[8], \\
& \Gamma_{c_{3}}^{\mathrm{fin} .}=\frac{1}{27} C^{2}\left(\frac{141}{32} \stackrel{\circ}{M_{[8]}^{-1}}-4 d_{D}\right)+\frac{1}{32} g_{F}^{(V)} \stackrel{\circ}{M_{[8]}} \text {, }
\end{aligned}
$$




$$
\begin{aligned}
& \Gamma_{c_{4}}^{\text {fin. }}=\frac{1}{81} C^{2}\left(47 \stackrel{\circ}{M}_{[8]}^{-1}-4\left(9 d_{0}+8 d_{D}\right)\right)-\frac{1}{144}\left(9 g_{1}^{(V)}+13 g_{D}^{(V)}\right) \stackrel{\circ}{M}_{[8]}, \\
& \Gamma_{c_{5}}^{\text {fin. }}=-\frac{1}{81} C^{2}\left(\frac{611}{16} \stackrel{\circ}{M}_{[8]}^{-1}-2\left(15 d_{0}+19 d_{D}\right)\right)-\frac{13}{144} g_{F}^{(V)} \stackrel{\circ}{M}_{[8]}, \\
& \Gamma_{c_{6}}^{\text {fin. }}=-\frac{1}{81} C^{2}\left(\frac{1645}{32} \stackrel{\circ}{M}_{[8]}^{-1}-2\left(42 d_{0}+19 d_{D}\right)\right)-\frac{1}{864}\left(66 g_{0}^{(V)}-27 g_{1}^{(V)}-8 g_{D}^{(V)}\right) \stackrel{\circ}{M}[8] .
\end{aligned}
$$

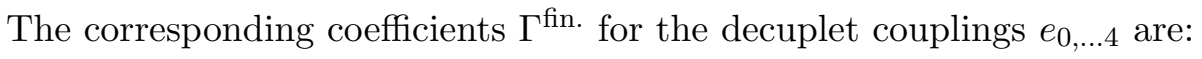

$$
\begin{aligned}
& \Gamma_{e_{0}}^{\text {fin. }}=\frac{1}{9} C^{2}\left(\frac{39}{32} \stackrel{\circ}{M}_{[8]}^{-1}+4\left(b_{D}-b_{F}\right)\right)-\frac{1}{27} H^{2}\left(\frac{91}{8} \stackrel{\circ}{M}_{[8]}^{-1}-\frac{32}{9} d_{D}\right) \\
& -\frac{1}{144}\left[\left(15 h_{2}^{(S)}+13 h_{4}^{(S)}+9 h_{6}^{(S)}\right)+\left(15 h_{1}^{(V)}+13 h_{2}^{(V)}+9 h_{3}^{(V)}\right) \stackrel{\circ}{M}[8]\right], \\
& \Gamma_{e_{1}}^{\text {fin. }}=\frac{1}{9} C^{2}\left(\frac{13}{16} \stackrel{\circ}{M_{[8]}^{-1}}+8 b_{F}\right)+\frac{1}{27} H^{2}\left(\frac{13}{2} \stackrel{\circ}{\stackrel{\circ}{M}_{[8]}^{-1}}-\frac{64}{3} d_{D}\right) \\
& -\frac{1}{24}\left(h_{6}^{(S)}+h_{3}^{(V)} \stackrel{\circ}{M}[8]\right) \text {, } \\
& \Gamma_{e_{2}}^{\text {fin. }}=-\frac{1}{3} C^{2}\left(\frac{13}{32} \stackrel{\circ}{M_{[8]}^{-1}}+\frac{8}{3} b_{D}\right)+\frac{1}{9} H^{2}\left(\frac{13}{8} \stackrel{\circ}{M}_{[8]}^{-1}+\frac{64}{9} d_{D}\right) \\
& +\frac{1}{16}\left[\left(h_{4}^{(S)}+h_{6}^{(S)}\right)+\left(h_{2}^{(V)}+h_{3}^{(V)}\right) \stackrel{\circ}{M_{[8]}}\right] \\
& \Gamma_{e_{3}}^{\text {fin. }}=\frac{1}{3} C^{2}\left(\frac{13}{16} \stackrel{\circ}{M}_{[8]}^{-1}-\frac{4}{3}\left(b_{0}+2 b_{F}\right)\right)-\frac{1}{27} H^{2}\left(\frac{91}{4} \stackrel{\circ}{M}_{[8]}^{-1}-\frac{32}{9}\left(9 d_{0}+5 d_{D}\right)\right) \\
& -\frac{1}{72}\left[\left(13 h_{4}^{(S)}+9 h_{6}^{(S)}\right)+\left(13 h_{2}^{(V)}+9 h_{3}^{(V)}\right) \stackrel{\circ}{M}_{[8]}\right] \text {, } \\
& \Gamma_{e_{4}}^{\text {fin. }}=-\frac{4}{9} C^{2}\left(b_{0}+b_{D}-b_{F}\right)+\frac{1}{81} H^{2}\left(\frac{13}{2} \stackrel{\circ}{M}[8]+\frac{160}{3} d_{0}\right) \\
& -\frac{1}{432}\left[\left(33 h_{2}^{(S)}-4 h_{4}^{(S)}\right)+\left(33 h_{1}^{(V)}-4 h_{2}^{(V)}\right) \stackrel{\circ}{M}[8]\right] \text {. }
\end{aligned}
$$

\section{A.4. Redundancy of the off-shell parameter $Z$}

The description of the interacting spin-3/2 fields leads to the introduction of an additional off-shell parameter for each spin-3/2 field. The off-shell parameter describes to which amount the lower-spin component of the field operator contribute to the physical observables (see e.g. [69]). In the framework of the effective field theory these off-shell parameters can be absorbed, independent of whether they can be fixed by theoretical arguments or by fitting to the experemental data or not, by the contact interaction terms which are of higher order in the energy expansion $[86,58]$. This redundance can also be understood in terms of a suitable field redefinition, which eliminates the contribution of the lower-spin component in the original interaction at the cost of the introduction of additional interaction terms [74].

In the following we consider a derivative coupling of a scalar spin- 0 field $\phi$ to a spin- $1 / 2$ field $\psi$ and a spin-3/2 Rarita-Schwinger field $\psi_{\mu}$ with a minimal number of derivatives. 
The associated masses are $m, M$ and $M_{\frac{3}{2}}$. We apply the ideas of Tang and Ellis in [86] and generalize them to the $S U(3)$ chiral version of this vertex and to the case of arbitrary number of dimensions $d^{1}$.

Starting with the tree-point vertex

$$
\mathcal{L}=C\left(\bar{\psi}_{\mu} \Theta^{\mu \nu} \psi \partial_{\nu} \phi+\text { h.c. }\right), \quad \Theta_{\mu \nu}=g_{\mu \nu}-\frac{1}{2} Z \gamma_{\mu} \gamma_{\nu}
$$

( $C$ is the dimensionful coupling, $Z$ is the off-shell parameter) the $s$ - and $u$-channel diagrams with the exchange of the spin- $3 / 2$ particle can be obtained by means of the following effective four-point interaction:

$$
\mathcal{L}^{e f f}=-C^{2} \bar{\psi} \Theta_{\mu \lambda} S^{\lambda \sigma} \Theta_{\sigma \nu} \psi \partial^{\mu} \phi \partial^{\nu} \phi
$$

with

$$
S_{\mu \nu}=\frac{-1}{i \not \partial-M_{\frac{3}{2}}+i \varepsilon}\left(g_{\mu \nu}-\frac{1}{d-1} \gamma_{\mu} \gamma_{\nu}-\frac{i}{(d-1) M_{\frac{3}{2}}}\left(\gamma_{\mu} \partial_{\nu}-\gamma_{\nu} \partial_{\mu}\right)-\frac{d-2}{(d-1) M_{\frac{3}{2}}^{2}} \partial_{\mu} \partial_{\nu}\right) \text {. }
$$

The spin-3/2 propagator in the coordinate space

$$
i S_{\mu \nu}(x-y)=\int \frac{d^{4} k}{(2 \pi)^{4}} i S_{\mu \nu} e^{i k(x-y)}
$$

satisfies the equation

$$
\Lambda^{\mu \sigma} S_{\sigma \nu}(x-y)=-\delta^{(4)}(x-y) g_{\nu}^{\mu},
$$

$\Lambda_{\mu \nu}$ being the differential operator that determines the kinetic term of the spin- $3 / 2$ field in the Lagrangian:

$$
\Lambda_{\mu \nu}=-\left(i \not \partial-M_{\frac{3}{2}}\right) g_{\mu \nu}+i\left(\gamma_{\mu} \partial_{\nu}+\gamma_{\nu} \partial_{\mu}\right)-\gamma_{\mu}\left(i \not \partial+M_{\frac{3}{2}}\right) \gamma_{\nu}
$$

Using the property

$$
\gamma^{\mu} S_{\mu \nu}=S_{\nu \mu} \gamma^{\mu}=\frac{1}{(d-1) M_{\frac{3}{2}}}\left(\frac{d-2}{M_{\frac{3}{2}}} i \partial_{\nu}-\gamma_{\nu}\right),
$$

and

$$
\begin{aligned}
\Theta_{\mu \lambda} S^{\lambda \sigma} \Theta_{\sigma \nu} & =S_{\mu \nu}-\frac{Z^{2}(d-2)}{4(d-1) M_{\frac{3}{2}}^{2}}\left(g_{\mu \nu}-i \sigma_{\mu \nu}\right) i \not \partial-\frac{Z(Z d-4)}{4(d-1) M_{\frac{3}{2}}}\left(g_{\mu \nu}-i \sigma_{\mu \nu}\right) \\
& +\frac{Z^{2}(d-2)}{2(d-1) M_{\frac{3}{2}}^{2}} \gamma_{\mu} i \partial_{\nu}-\frac{Z(d-2)}{2(d-1) M_{\frac{3}{2}}^{2}}\left(i \partial_{\nu} \gamma_{\mu}+\gamma_{\nu} i \partial_{\mu}\right)
\end{aligned}
$$

\footnotetext{
${ }^{1}$ The analysis can be carried out for other vertices linear in spin-3/2 fields
} 
the effective Lagrangian in (A.17) can now be written as

$$
\begin{aligned}
\mathcal{L}^{e f f} & =-C^{2} \bar{\psi} S^{\mu \nu} \psi \partial_{\mu} \phi \partial_{\nu} \phi \\
& +R^{(S, T)}\left(\bar{\psi} \psi \partial^{\mu} \phi \partial_{\mu} \phi-\bar{\psi} i \sigma^{\mu \nu} \psi \partial_{\mu} \phi \partial_{\nu} \phi\right) \\
& +R^{(\$, T)}\left(\bar{\psi} i \not \partial \psi \partial^{\mu} \phi \partial_{\mu} \phi-\bar{\psi} i \sigma^{\mu \nu} i \not \partial \psi \partial_{\mu} \phi \partial_{\nu} \phi\right) \\
& +\frac{1}{2} R^{(V)}\left(\bar{\psi} \gamma_{\mu} i \partial_{\nu} \psi \partial^{\mu} \phi \partial^{\nu} \phi+\text { h.c. }\right) \\
& =-C^{2} \bar{\psi} S^{\mu \nu} \psi \partial_{\mu} \phi \partial_{\nu} \phi \\
& \left.+\left[R^{(S, T)}+M R^{(\$, ~}\right)\right]\left(\bar{\psi} \psi \partial^{\mu} \phi \partial_{\mu} \phi-\bar{\psi} i \sigma^{\mu \nu} \psi \partial_{\mu} \phi \partial_{\nu} \phi\right) \\
& +\frac{1}{2} R^{(V)}\left(\bar{\psi} \gamma_{\mu} i \partial_{\nu} \psi \partial^{\mu} \phi \partial^{\nu} \phi+\text { h.c. }\right) \\
& +R^{(\$, T)}\left(\bar{\psi}(i \not \partial-M) \psi \partial^{\mu} \phi \partial_{\mu} \phi-\bar{\psi} i \sigma^{\mu \nu}(i \not \partial-M) \psi \partial_{\mu} \phi \partial_{\nu} \phi\right),
\end{aligned}
$$

with

$$
\begin{gathered}
R^{(S, T)}=C^{2} \frac{Z(Z d-4)}{4(d-1) M_{\frac{3}{2}}}, \quad R^{(\$, F)}=C^{2} \frac{Z^{2}(d-2)}{4(d-1) M_{\frac{3}{2}}^{2}}, \\
R^{(V)}=-C^{2} \frac{Z(Z-2)(d-2)}{2(d-1) M_{\frac{3}{2}}^{2}} .
\end{gathered}
$$

The $Z$-dependent terms in (A.24) can be absorbed by the $Q^{2}$ interaction terms of the form

$$
\begin{aligned}
\mathcal{L}^{(2)} & =g^{(S)} \bar{\psi} \psi \partial^{\mu} \phi \partial_{\mu} \phi+g^{(\phi)} \bar{\psi} i \not \partial \psi \partial^{\mu} \phi \partial_{\mu} \phi \\
& +g^{(T)} \bar{\psi} i \sigma^{\mu \nu} \psi \partial_{\mu} \phi \partial_{\nu} \phi+g^{(T)} \bar{\psi} i \sigma^{\mu \nu} i \not \partial \psi \partial_{\mu} \phi \partial_{\nu} \phi \\
& +\frac{1}{2} g^{(V)}\left(\bar{\psi} \gamma_{\mu} i \partial_{\nu} \psi \partial^{\mu} \phi \partial^{\nu} \phi+\text { h.c. }\right) \\
& =\left[g^{(S)}+M g^{(\phi)}\right] \bar{\psi} \psi \partial^{\mu} \phi \partial_{\mu} \phi+\left[g^{(T)}+M g^{(F)}\right] \bar{\psi} i \sigma^{\mu \nu} \psi \partial_{\mu} \phi \partial_{\nu} \phi \\
& +\frac{1}{2} g^{(V)}\left(\bar{\psi} \gamma_{\mu} i \partial_{\nu} \psi \partial^{\mu} \phi \partial^{\nu} \phi+\text { h.c. }\right) \\
& +g^{(\phi)} \bar{\psi}(i \not \partial-M) \psi \partial^{\mu} \phi \partial_{\mu} \phi+g^{(T)} \bar{\psi} i \sigma^{\mu \nu}(i \not \partial-M) \psi \partial_{\mu} \phi \partial_{\nu} \phi .
\end{aligned}
$$

Upon the redefinition

$$
g^{(S)} \rightarrow g^{(S)}+M g^{(\$)}, \quad g^{(T)} \rightarrow g^{(T)}+M g^{(T)},
$$

the absorption of the dependence on the off-shell parameter $Z$ in the vertex (A.16) amounts to a finite renormalisation of the $Q^{2}$ couplings in the above Lagrangian according to

$$
\begin{aligned}
\delta g^{(S)} & =R^{(S, T)}+M R^{(\$, T)} \\
\delta g^{(V)} & =R^{(V)} \\
\delta g^{(T)} & =-\left(R^{(S, T)}+M R^{(\$, T)}\right) .
\end{aligned}
$$


At one loop-level, the $Z$-dependent part in the contribution to the self-energy of the spin-1/2 particle, $\Sigma^{Z}$, calculated with (A.16), is $Q^{4}$ and can be fully absorbed into the contributions from the $Q^{4}$ tadpole diagrams, $\Sigma^{(4)}$, calculated with (A.26). The explicit form of the both contributions in dimensional regularisation is

$$
\begin{aligned}
\Sigma^{Z}(p) & =\int \frac{d^{d} k}{(2 \pi)^{d}} \frac{i \mu_{U V}^{4-d}}{k^{2}-m^{2}+i \varepsilon}\left[C^{2} k_{\mu} \Theta^{\mu \lambda} S_{\lambda \sigma}(p-k) \Theta^{\sigma \nu} k_{\nu}-C^{2} k_{\mu} S^{\mu \nu}(p-k) k_{\nu}\right] \\
& =-\int \frac{d^{d} k}{(2 \pi)^{d}} \frac{i \mu_{U V}^{4-d}}{k^{2}-m^{2}+i \varepsilon}\left[R^{(S)} k^{2}+R^{(\$)} \not p k^{2}+R^{(V)} \not k(k \cdot p)\right] \\
& =-\int \frac{d^{d} k}{(2 \pi)^{d}} \frac{i \mu_{U V}^{4-d}}{k^{2}-m^{2}+i \varepsilon}\left[R^{(S)}+\not p\left(R^{(\$)}+\frac{1}{d} R^{(V)}\right)\right] m^{2}, \\
& =-\int \frac{d^{d} k}{(2 \pi)^{d}} \frac{i \mu_{U V}^{4-d}}{k^{2}-m^{2}+i \varepsilon} \\
\Sigma^{(4)}(p) & =-\int \frac{d^{d} k}{(2 \pi)^{d}} \frac{i \mu_{U V}^{4-d}}{k^{2}-m^{2}+i \varepsilon}\left[g^{(S)} k^{2}+g^{(\$)}(\not p-M) k^{2}+g^{(V)} \not k(k \cdot p)\right] \\
& =-\int \frac{d^{d} k}{(2 \pi)^{d}} \frac{i \mu_{U V}^{4-d}}{k^{2}-m^{2}+i \varepsilon}\left[g^{(S)}+g^{(\$)}(\not p-M)+\not p \frac{1}{d} g^{(V)}\right] m^{2} \\
& =-\int \frac{d^{d} k}{(2 \pi)^{d}} \frac{i \mu_{U V}^{4-d}}{k^{2}-m^{2}+i \varepsilon}\left[\left(g^{(S)}+\frac{1}{d} M g^{(V)}\right)+(\not p-M)\left(g^{(\$)}+\frac{1}{d} g^{(V)}\right)\right] m^{2} .
\end{aligned}
$$

At one-loop level we observe that the expressions (A.29) and (A.30) preclude one from drawing the conclusions about the renormalisation of each coupling constant in (A.26) separately. However, it is possible, as expected, to absorb all the $Z$-dependence into the tadpole contributions in $\Sigma^{(4)}$ in complience with the results in (A.28).

The results in (A.24) and (A.28) are generalised to the $S U(3)$ invariant interaction by mapping the flavour structure in the $S U(3)$ version of (A.24) to the flavour basis defining the flavour structures of the $Q^{2}$ terms stated in Section 1.4. It holds:

$$
\begin{aligned}
& \delta g_{0}^{(S)}=\frac{4}{3}\left(R^{(S, T)}+R^{(\$, 7)}\right), \quad \delta g_{1}^{(S)}=-\frac{1}{3}\left(R^{(S, T)}+R^{(\$, 7)}\right), \\
& \delta g_{D}^{(S)}=-\left(R^{(S, T)}+R^{(\$, 7)}\right), \quad \delta g_{F}^{(S)}=R^{(S, T)}+R^{(\$, F)}, \\
& \delta g_{0}^{(V)}=\frac{4}{3} R^{(V)}, \quad \delta g_{1}^{(V)}=-\frac{1}{3} R^{(V)}, \\
& \delta g_{D}^{(V)}=-R^{(V)}, \quad \delta g_{F}^{(V)}=R^{(V)},
\end{aligned}
$$




$$
\begin{gathered}
\delta g_{1}^{(T)}=R^{(S, T)}+R^{(\$, F)}, \quad \delta g_{D}^{(T)}=-\left(R^{(S, T)}+R^{(\$, T)}\right), \\
\delta g_{F}^{(T)}=-\frac{1}{3}\left(R^{(S, T)}+R^{(\$, F)}\right) .
\end{gathered}
$$




\section{Appendix B.}

\section{$S U(3)$ group theory}

The importance of the $S U(3)$ Lie-group in physics is subject of many textbooks (see e.g. $[33,59,44])$. In this chapter the main properties of the generators of the group transformations are summarised ${ }^{1}$. Furthermore, the decomposition of the product of two arbitrary $S U(3)$ octets is discussed.

The main properties of the generators of the $S U(3)$ group

$$
t^{a}=\frac{1}{2} \lambda^{a}, \quad(a=1, \ldots 8)
$$

$\lambda^{a}$ being the Gell-Mann matrices

$$
\begin{gathered}
\lambda^{1}=\left(\begin{array}{lll}
0 & 1 & 0 \\
1 & 0 & 0 \\
0 & 0 & 0
\end{array}\right), \quad \lambda^{2}=\left(\begin{array}{ccc}
0 & -i & 0 \\
i & 0 & 0 \\
0 & 0 & 0
\end{array}\right), \quad \lambda^{3}=\left(\begin{array}{ccc}
1 & 0 & 0 \\
0 & -1 & 0 \\
0 & 0 & 0
\end{array}\right), \\
\lambda^{4}=\left(\begin{array}{lll}
0 & 0 & 1 \\
0 & 0 & 0 \\
1 & 0 & 0
\end{array}\right), \quad \lambda^{5}=\left(\begin{array}{ccc}
0 & 0 & -i \\
0 & 0 & 0 \\
i & 0 & 0
\end{array}\right), \quad \lambda^{6}=\left(\begin{array}{ccc}
0 & 0 & 0 \\
0 & 0 & 1 \\
0 & 1 & 0
\end{array}\right), \\
\lambda^{7}=\left(\begin{array}{ccc}
0 & 0 & 0 \\
0 & 0 & -i \\
0 & i & 0
\end{array}\right), \quad \lambda^{8}=\frac{1}{\sqrt{3}}\left(\begin{array}{ccc}
1 & 0 & 0 \\
0 & 1 & 0 \\
0 & 0 & -2
\end{array}\right),
\end{gathered}
$$

are

$$
\begin{gathered}
t^{a \dagger}=t^{a}, \quad \operatorname{tr}\left(t^{a}\right)=0, \quad \operatorname{tr}\left(t^{a} t^{b}\right)=\frac{1}{2} \delta^{a b}, \\
{\left[t^{a}, t^{b}\right]=i f^{a b c} t^{c}, \quad\left\{t^{a}, t^{b}\right\}=\frac{1}{3} \delta^{a b}+d^{a b c} t^{c} .}
\end{gathered}
$$

From this, one obtains for the product of two generators

$$
t^{a} t^{b}=\frac{1}{6} \delta^{a b}+\frac{1}{2}\left(d^{a b c}+i f^{a b c}\right) t^{c}
$$

${ }^{1}$ For further relations we refer to the mentioned textbooks or to [13]. 
and the $d$ - and $f$-symbols of the $S U(3)$ group are given by

$$
d^{a b c}=2 \operatorname{tr}\left(t^{a}\left\{t^{b}, t^{c}\right\}\right), \quad f^{a b c}=-2 i \operatorname{tr}\left(t^{a}\left[t^{b}, t^{c}\right]\right) .
$$

In general, tensors or products of them are reducible in the sence that they can be further decomposed into components, which transforms under different irreducible representations of the symmetry group. Irreducible representations can be specified by the dimension of the multiplet - orthonormal basis of the support of the representation.

Working with the effective quark operators in Sections 2.3.1 and 2.3.2 it is more appropriate to use $S U(3)$ flavour octets described by a single adjoint index $a=1, \ldots 8$ instead of the indices $i, j=1,2,3$ that are used to label vectors transforming under the fundamental representation. Given a general octet tensor $O_{i}^{j}$, the connection is established via:

$$
O^{a}=\left(t^{a}\right)_{j}^{i} O_{i}^{j}, \quad \text { and } \quad O_{j}^{i}=2 O^{a}\left(t^{a}\right)_{j}^{i} .
$$

In the following, the decomposition of the product of two arbitrary octets, $A_{j}^{i}$ and $B_{j}^{i}$, is discussed. It holds:

$$
\mathbf{8} \otimes \mathbf{8}=\mathbf{1} \oplus \mathbf{8}_{S} \oplus \mathbf{8}_{A} \oplus \mathbf{1 0} \oplus \overline{\mathbf{1 0}} \oplus \mathbf{2 7}
$$

The singlet, $\mathbf{1}$, is obtained by contracting all indices:

$$
S=A_{l}^{i} B_{i}^{l}=4 A^{a} B^{b} \operatorname{tr}\left(t^{a} t^{b}\right)=2 A^{a} B^{a} .
$$

The symmetric octet, $\mathbf{8}_{\mathbf{S}}$, in the product of two octets:

$$
\begin{aligned}
D_{j}^{i} & =A_{k}^{i} B_{j}^{k}+B_{k}^{i} A_{j}^{k}-\frac{2}{3} \delta_{j}^{i} A_{l}^{k} B_{k}^{l}=4 A^{a} B^{b}\left\{t^{a}, t^{b}\right\}_{j}^{i}-\frac{2}{3} \delta_{j}^{i} 4 A^{a} B^{b} \operatorname{tr}\left(t^{a} t^{b}\right) \\
& =4 A^{a} B^{b}\left(\frac{1}{3} \delta^{a b}+d^{a b c} t^{c}\right)_{j}^{i}-\frac{2}{3} \delta_{j}^{i} 4 A^{a} B^{b} \frac{1}{2} \delta^{a b}=4 A^{a} B^{b} d^{a b c}\left(t^{c}\right)_{j}^{i} .
\end{aligned}
$$

The symmetric octet, described by a single adjoint index, is given by

$$
D^{c}=\left(t^{c}\right)_{j}^{i} D_{i}^{j}=2 d^{a b c} A^{a} B^{b}=d^{a b c}\left(A^{a} B^{b}+A^{b} B^{a}\right) .
$$

Similar for the antisymmetric octet, $\mathbf{8}_{\mathbf{A}}$ :

$$
F_{j}^{i}=A_{k}^{i} B_{j}^{k}-B_{k}^{i} A_{j}^{k}=4 A^{a} B^{b}\left[t^{a}, t^{b}\right]_{j}^{i}=4 A^{a} B^{b} i f^{a b c}\left(t^{c}\right)_{j}^{i},
$$

and

$$
F^{c}=\left(t^{c}\right)_{j}^{i} F_{i}^{j}=2 i f^{a b c} A^{a} B^{b}=i f^{a b c}\left(A^{a} B^{b}-A^{b} B^{a}\right) .
$$

Decuplet and antidecuplet tensors in the product of two octets are:

$$
T^{i j k}=A_{l}^{i} B_{m}^{j} \epsilon^{k l m}+\operatorname{perm}(i j k), \quad T_{i j k}=A_{i}^{l} B_{j}^{m} \epsilon_{k l m}+\operatorname{perm}(i j k) .
$$

Another way to represent decuplet and anti-decuplet tensors uses the fact, that the totaly antisymmetric tensor of $S U(3), \varepsilon^{i j k}=\varepsilon_{i j k}$, carries three indices. A pair of upper indices 
in which the tensor is antisymmetric can be always traded for one lower index using an epsilon-tensor and similarly for the lower indices. It holds:

$$
\begin{aligned}
T_{[l m]}^{(i j)} & =A_{l}^{i} B_{m}^{j}+A_{l}^{j} B_{m}^{i}-A_{m}^{i} B_{l}^{j}-A_{m}^{j} B_{l}^{i} \\
& -\frac{1}{3}\left(\delta_{l}^{i} F_{m}^{j}-\delta_{m}^{i} F_{l}^{j}+\delta_{l}^{j} F_{m}^{i}-\delta_{m}^{j} F_{l}^{i}\right), \\
T_{(l m)}^{[i j]} & =A_{l}^{i} B_{m}^{j}+A_{m}^{i} B_{l}^{j}-A_{l}^{j} B_{m}^{i}-A_{m}^{j} B_{l}^{i} \\
& -\frac{1}{3}\left(-\delta_{l}^{i} F_{m}^{j}-\delta_{m}^{i} F_{l}^{j}+\delta_{l}^{j} F_{m}^{i}+\delta_{m}^{j} F_{l}^{j}\right) .
\end{aligned}
$$

Here, the round and square brackets on $T$ indicate the symmetrisation and the antisymmetrisation of the indices, respectively, and the subtraction of F's, defined in (B.11), is required to make the tensors traceless. Converting the sum of these multiplets, $\mathbf{1 0}+\overline{\mathbf{1 0}}$,

$$
T_{[l m]}^{(i j)}+T_{(l m)}^{[i j]}=2\left(A_{l}^{i} B_{m}^{j}-A_{m}^{j} B_{l}^{i}-\frac{1}{3}\left(\delta_{l}^{j} F_{m}^{i}-\delta_{m}^{i} F_{l}^{j}\right)\right)
$$

to an object with two adjoint indices yields upon a short calculation:

$$
T^{a b}=\left(t^{a}\right)_{i}^{l}\left(t^{b}\right)_{j}^{m}\left(T_{[l m]}^{(i j)}+T_{(i j)}^{[l m]}\right)=2\left(A^{a} B^{b}-A^{b} B^{a}-\frac{2}{3} f^{a b c} f^{c d e} A^{d} B^{e}\right) .
$$

Finally, 27 in $A_{l}^{i} B_{m}^{j}$ is obtained by symmetrizing upper and lower indices and by making this tensor traceless:

$$
\begin{aligned}
I_{(l m)}^{(i j)} & =A_{l}^{i} B_{m}^{j}+A_{l}^{j} B_{m}^{i}+A_{m}^{i} B_{l}^{j}+A_{m}^{j} B_{l}^{i} \\
& -\frac{1}{5}\left(\delta_{l}^{i} D_{m}^{j}+\delta_{l}^{j} D_{m}^{i}+\delta_{m}^{i} D_{l}^{j}+\delta_{m}^{j} D_{l}^{i}\right)-\frac{1}{6}\left(\delta_{l}^{i} \delta_{m}^{j}+\delta_{l}^{j} \delta_{m}^{i}\right) S .
\end{aligned}
$$

Here, the subtracted terms contain the symmetric octet and singlet tensors, defined in (B.9) and (B.8), respectively. The 27-plet, described by two adjoint indices, is given by

$$
I^{a b}=\left(t^{a}\right)_{j}^{l}\left(t^{b}\right)_{j}^{m} I_{(l m)}^{(i j)}=2\left(A^{a} B^{b}+A^{b} B^{a}-\frac{6}{5} d^{a b c} d^{c g h} A^{g} B^{h}-\frac{1}{4} \delta^{a b} A^{c} B^{c}\right) .
$$

We summarise the results of this section in a form, which is well suited for the discussion of the identities for the effective quark operators in Appendix G.

In general, a tensor with two adjoint flavour indices, $\chi^{a b}$, can be decomposed into symmetric and antisymmetric parts:

$$
\chi^{a b}=\frac{1}{2}\left(\chi_{+}^{a b}+\chi_{-}^{a b}\right)
$$

Further decomposition of these parts into the different multiplets is obtained by using 
the results in (B.8, B.10, B.12, B.16, B.18):

$$
\begin{aligned}
\chi_{+}^{a b} & =\frac{1}{8} \delta^{a b} \chi_{+}^{c c}+\frac{3}{5} d^{a b c} d^{c g h} \chi_{+}^{g h}+\left(\chi_{+}^{a b}-\frac{1}{8} \delta^{a b} \chi_{+}^{c c}-\frac{3}{5} d^{a b c} d^{c g h} \chi_{+}^{g h}\right) \\
& =\frac{1}{8} \delta^{a b} \chi_{1}+\frac{3}{5} d^{a b c} \chi_{8_{S}}^{c}+\chi_{27}^{a b}, \\
\chi_{-}^{a b} & =\frac{1}{3} f^{a b c} f^{c g h} \chi_{-}^{g h}+\left(\chi_{-}^{a b}-\frac{1}{3} f^{a b c} f^{c g h} \chi_{-}^{g h}\right) \\
& =\frac{1}{3} f^{a b c} \chi_{8_{A}}^{c}+\chi_{10+10}^{a b},
\end{aligned}
$$

with the symmetric components

$$
\chi_{1}=\chi_{+}^{c c}, \quad \chi_{8_{S}}^{c}=d^{a b c} \chi_{+}^{a b}, \quad \chi_{27}^{a b}=\chi_{+}^{a b}-\frac{1}{8} \delta^{a b} \chi_{+}^{c c}-\frac{3}{5} d^{a b c} d^{c g h} \chi_{+}^{g h},
$$

and with the antisymmetric components

$$
\chi_{8_{A}}^{c}=f^{a b c} \chi_{-}^{a b}, \quad \chi_{10+10}^{a b}=\chi_{-}^{a b}-\frac{1}{3} f^{a b c} f^{c g h} \chi_{-}^{g h} .
$$




\section{Appendix C.}

\section{Interaction}

When dealing with effective field theories, where the degrees of freedom are described by $S U(3)$ flavour tensors ${ }^{1}$, one is confronted with the problem of finding the minimal number of linearly independent $S U(3)$-invariant terms. This number is obtained by counting the singlets in the decomposition of the outer product of the $S U(3)$ tensors under consideration. To determine the terms themselves, a general method for octets only was formulated in [24].

The first section of this appendix presents group theoretical methods, similar in spirit to [24], for the construction of flavour structures that are needed to fully describe the $Q^{2}$ four-point meson-baryon interaction which is stated in Section 1.4.2. Results obtained for this vertex are then applied with small modifications to the construction of $Q^{4}$-terms in Section 1.4.3 that break the chiral symmetry explicitly. The analysis is carried out for octet and decuplet baryons.

The second section discusses transformation properties under charge conjugation of the building blocks of the four-point meson-baryon interaction.

\section{C.1. SU(3)-invariants}

Following [24], in order to determine the invariant terms for a vertex containing $n$ octets $O=\sum_{a} o_{i}^{a} \lambda^{(a)},(i=1, \ldots n)$, one constructs a set of independent tensors of rank $n$ and contract them with $o_{i}^{a}$ to obtain singlets. To be more definite, we consider first the familiar case of the three-point meson-baryon vertex $\bar{B} B \Phi$. The octets - the antibaryon matrix $\bar{B}$, the baryon matrix $B$ and the meson matrix $\Phi$ - are given by

$$
\bar{B}=\frac{1}{\sqrt{2}} \bar{b}^{a} \lambda^{a}, \quad B=\frac{1}{\sqrt{2}} b^{a} \lambda^{a}, \quad \text { and } \quad \Phi=\phi^{a} \lambda^{a},
$$

respectively. There are only two linearly independent tensors of the 3rd-rank $-d^{a b c}$ and $f^{a b c}$. $S U(3)$-invariant terms are obtained by contracting the open indices on these tensors with the open indices on octets in (C.1):

$$
\bar{b}^{a} b^{b} \phi^{c} d^{a b c} \sim \operatorname{tr}\left(\bar{B}[B, \Phi]_{+}\right), \quad \bar{b}^{a} b^{b} \phi^{c} f^{a b c} \sim \operatorname{tr}\left(\bar{B}[B, \Phi]_{-}\right),
$$

${ }^{1}$ The discussion here, though applicable to an arbitrary number of flavours $N_{F}$, is limited to $N_{F}=3$. 
Another way to obtain these terms is the explicit construction of all possible singlet contractions. The decomposition of the product of three octets is

$$
\mathbf{8} \otimes(\mathbf{8} \otimes \mathbf{8})=\mathbf{8} \otimes\left(\mathbf{1} \oplus \mathbf{8}_{S} \oplus \mathbf{8}_{A} \oplus \mathbf{1 0} \oplus \overline{\mathbf{1 0}} \oplus \mathbf{2 7}\right) .
$$

Contractions leading to a singlet are easily obtained by using the explicit expressions for the multiplets on the right hand side. The only invariant terms stem from the contractions of $\mathbf{8}$ with $\mathbf{8}_{A}$ and $\boldsymbol{8}_{S}$ to a singlet:

$$
\begin{aligned}
\bar{B}_{j}^{i}\left(B_{k}^{j} \Phi_{i}^{k}-\Phi_{k}^{j} B_{i}^{k}\right) & =\operatorname{tr}\left(\bar{B}[B, \Phi]_{-}\right), \\
\bar{B}_{j}^{i}\left(B_{k}^{j} \Phi_{i}^{k}+\Phi_{k}^{j} B_{i}^{k}-\frac{2}{3} \delta_{j}^{i} B_{k}^{l} \Phi_{l}^{k}\right) & =\operatorname{tr}\left(\bar{B}\left([B, \Phi]_{+}\right) .\right.
\end{aligned}
$$

We turn to the discussion of the flavour structure of the four-point interaction introduced in Section 1.4.2. Flavour structures of all invariant interaction terms containing octet baryons and mesons are obtained from the study of the product of four octets:

$$
(\mathbf{8} \otimes \mathbf{8}) \otimes(\mathbf{8} \otimes \mathbf{8})=\left(\mathbf{1} \oplus \mathbf{8}_{S} \oplus \mathbf{8}_{A} \oplus \mathbf{1 0} \oplus \overline{\mathbf{1 0}} \oplus \mathbf{2 7}\right) \otimes\left(\mathbf{1} \oplus \mathbf{8}_{S} \oplus \mathbf{8}_{A} \oplus \mathbf{1 0} \oplus \overline{\mathbf{1 0}} \oplus \mathbf{2 7}\right) .
$$

There are 8 singlets in this product:

$$
\begin{aligned}
& {[(\mathbf{8} \otimes \mathbf{8}) \otimes(\mathbf{8} \otimes \mathbf{8})]_{\text {singlets }}=[\mathbf{1} \otimes \mathbf{1}]_{\text {singlet }} \oplus\left[\mathbf{8}_{S, A} \otimes \mathbf{8}_{S, A}\right]_{\text {singlet }} \oplus[\mathbf{1 0} \otimes \overline{\mathbf{1 0}}]_{\text {singlet }}} \\
& \oplus[\overline{\mathbf{1 0}} \otimes \mathbf{1 0}]_{\text {singlet }} \oplus[\mathbf{2 7} \otimes \mathbf{2 7}]_{\text {singlet }} .
\end{aligned}
$$

The $S U(3)$-invariant terms are:

$$
\begin{aligned}
& {[\mathbf{1} \otimes \mathbf{1}]_{\text {singlet }}: \quad \operatorname{tr}(\bar{B} B) \operatorname{tr}\left(\Phi_{1} \Phi_{2}\right),} \\
& {\left[\mathbf{8}_{S} \otimes \mathbf{8}_{S}\right]_{\text {singlet }}: \quad \operatorname{tr}\left([\bar{B}, B]_{+}\left[\Phi_{1}, \Phi_{2}\right]_{+}\right)=\operatorname{tr}\left(\bar{B}\left[\left[\Phi_{1}, \Phi_{2}\right]_{+}, B\right]_{+}\right),} \\
& {\left[\mathbf{8}_{S} \otimes \mathbf{8}_{A}\right]_{\text {singlet }}: \quad \operatorname{tr}\left([\bar{B}, B]_{+}\left[\Phi_{1}, \Phi_{2}\right]_{-}\right)=\operatorname{tr}\left(\bar{B}\left[\left[\Phi_{1}, \Phi_{2}\right]_{-}, B\right]_{+}\right),} \\
& {\left[\mathbf{8}_{A} \otimes \mathbf{8}_{S}\right]_{\text {singlet }}: \quad \operatorname{tr}\left([\bar{B}, B]_{-}\left[\Phi_{1}, \Phi_{2}\right]_{+}\right)=-\operatorname{tr}\left(\bar{B}\left[\left[\Phi_{1}, \Phi_{2}\right]_{+}, B\right]_{-}\right),} \\
& {\left[\mathbf{8}_{A} \otimes \mathbf{8}_{A}\right]_{\text {singlet }}: \quad \operatorname{tr}\left([\bar{B}, B]_{-}\left[\Phi_{1}, \Phi_{2}\right]_{-}\right)=-\operatorname{tr}\left(\bar{B}\left[\left[\Phi_{1}, \Phi_{2}\right]_{-}, B\right]_{-}\right),} \\
& \left(\bar{B}_{l}^{i} B_{m}^{j} \varepsilon^{k l m}\right)^{\operatorname{sym}(k l m)}\left(\Phi_{1 i}^{o} \Phi_{2 j}^{p} \varepsilon_{i j k}\right)_{\text {sym }(k o p)}=-6 \operatorname{tr}\left(\bar{B}\left[\left[\Phi_{1}, \Phi_{2}\right]_{-}, B\right]_{-}\right) \\
& +18\left(\operatorname{tr}\left(\bar{B} \Phi_{1}\right) \operatorname{tr}\left(\Phi_{2} B\right)-\operatorname{tr}\left(\bar{B} \Phi_{2}\right) \operatorname{tr}\left(\Phi_{1} B\right)\right)+18\left(\operatorname{tr}\left(\bar{B} \Phi_{1} B \Phi_{2}\right)-\operatorname{tr}\left(\bar{B} \Phi_{2} B \Phi_{1}\right)\right) \text {, }
\end{aligned}
$$

$[\overline{\mathbf{1 0}} \otimes \mathbf{1 0}]_{\text {singlet }}:$

$$
\begin{aligned}
& \left(\bar{B}_{i}^{l} B_{j}^{m} \varepsilon_{k l m}\right)_{\text {sym }(k l m)}\left(\Phi_{o}^{1 i} \Phi_{p}^{2 j} \varepsilon^{i j k}\right)^{\text {sym }(\text { kop })}=-6 \operatorname{tr}\left(\bar{B}\left[\left[\Phi_{1}, \Phi_{2}\right]_{-}, B\right]_{-}\right) \\
& \quad+18\left(\operatorname{tr}\left(\bar{B} \Phi_{1}\right) \operatorname{tr}\left(\Phi_{2} B\right)-\operatorname{tr}\left(\bar{B} \Phi_{2}\right) \operatorname{tr}\left(\Phi_{1} B\right)\right)-18\left(\operatorname{tr}\left(\bar{B} \Phi_{1} B \Phi_{2}\right)-\operatorname{tr}\left(\bar{B} \Phi_{2} B \Phi_{1}\right)\right)
\end{aligned}
$$

$[\mathbf{2 7} \otimes \overline{\mathbf{2 7}}]_{\text {singlet }}:$

$$
\begin{aligned}
\left(\bar{B}_{l}^{i} B_{m}^{j}\right)_{\text {sym }(l m)}^{\operatorname{sym}(i j)}\left(\Phi_{1 i}^{l} \Phi_{2 j}^{m}\right)_{s y m(i j)}^{\operatorname{sym}(l m)} & \left.=4 \operatorname{tr}(\bar{B} B) \operatorname{tr}\left(\Phi_{1} \Phi_{2}\right)-4 \operatorname{tr}\left(\bar{B}\left[\Phi_{1}, \Phi_{2}\right]_{+}, B\right]_{+}\right) \\
& +8\left(\operatorname{tr}\left(\bar{B} \Phi_{1}\right) \operatorname{tr}\left(B \Phi_{2}\right)+\operatorname{tr}\left(\bar{B} \Phi_{2}\right) \operatorname{tr}\left(B \Phi_{1}\right)\right) .
\end{aligned}
$$


It should be stressed at this point, that the given contractions here do not always represent pure multiplets. Those would be obtained by additional subtractions of the singlet components, $\mathbf{1}$, in the symmetric octet contractions, $\mathbf{8}_{\mathrm{S}}$, and of the $\mathbf{1}$ and $\mathbf{8}_{\mathrm{S}}$ in the 27 plet contractions (see Appendix B for further explanations). For the sake of a more clear representation, such subtractions were not shown in (C.7) explicitly. Though the expressions on the r.h.s in (C.7) do not reflect the group structure exactly, building of the chiral Lagrangian out of them is fully sufficient, since they contain all relevant $S U(3)$-invariant flavour contractions.

From (C.7), the minimal set of terms which provide a complete basis in the flavour space for the $\bar{B} B \Phi_{1} \Phi_{2}$ vertex is readily written down:

$$
\begin{gathered}
\operatorname{tr}(\bar{B} B) \operatorname{tr}\left(\Phi_{1} \Phi_{2}\right), \quad \operatorname{tr}\left(\bar{B}\left[\left[\Phi_{1}, \Phi_{2}\right]_{ \pm}, B\right]_{+}\right), \quad \operatorname{tr}\left(\bar{B}\left[\left[\Phi_{1}, \Phi_{2}\right]_{ \pm}, B\right]_{-}\right), \\
\operatorname{tr}\left(\bar{B} \Phi_{1}\right) \operatorname{tr}\left(\Phi_{2} B\right) \pm \operatorname{tr}\left(\bar{B} \Phi_{2}\right) \operatorname{tr}\left(\Phi_{1} B\right), \\
\operatorname{tr}\left(\bar{B} \Phi_{1} B \Phi_{2}\right)-\operatorname{tr}\left(\bar{B} \Phi_{2} B \Phi_{1}\right) .
\end{gathered}
$$

The forgoing discussion is easily extended to the analogous four-point interaction containing the decuplet baryons. We discuss first the $S U(3)$-invariant terms in the product

$$
(\overline{\mathbf{1 0}} \otimes \mathbf{1 0}) \otimes(\mathbf{8} \otimes \mathbf{8})=(\mathbf{1} \oplus \mathbf{8} \oplus \mathbf{2 7} \oplus \mathbf{6 4}) \otimes\left(\mathbf{1} \oplus \mathbf{8}_{S} \oplus \mathbf{8}_{A} \oplus \mathbf{1 0} \oplus \overline{\mathbf{1 0}} \oplus \mathbf{2 7}\right) .
$$

The singlets in this product are $^{2}$

$$
\begin{aligned}
{[(\overline{\mathbf{1 0}} \otimes \mathbf{1 0})} & \otimes(\mathbf{8} \otimes \mathbf{8})]_{\text {singlets }} \\
= & {[\mathbf{1} \otimes \mathbf{1}]_{\text {singlet }} \oplus\left[\mathbf{8} \otimes \mathbf{8}_{S}\right]_{\text {singlet }} \oplus\left[\mathbf{8} \otimes \mathbf{8}_{A}\right]_{\text {singlet }} \oplus[\mathbf{2 7} \otimes \mathbf{2 7}]_{\text {singlet }} . }
\end{aligned}
$$

To simplify the notation in the following, we abbreviate various flavour contractions in the products of octets, decuplets and anti-decuplets as in

$$
\begin{aligned}
& \Phi_{1 l}^{m} \Phi_{2 k}^{l} \equiv\left(\Phi_{1} \cdot \Phi_{2}\right)_{k}^{m}, \quad \Phi_{1 l}^{j} \Phi_{2 m}^{k} \equiv\left\lfloor\Phi_{1} \cdot \Phi_{2}\right]_{l m}^{j k} \quad \Phi_{m}^{j} \Phi_{n}^{k} \varepsilon^{m n l} \equiv\langle\Phi \cdot \Phi\rangle^{j k l}, \\
& \bar{\Delta}_{i j k} \Delta^{i j m} \equiv(\bar{\Delta} \cdot \Delta)_{k}^{m}, \quad \bar{\Delta}_{i j k} \Delta^{i l m} \equiv\lfloor\bar{\Delta} \cdot \Delta\rceil_{j k}^{l m}, \quad \bar{\Delta}_{i j k} B_{l}^{i} \equiv\langle\bar{\Delta} \cdot B\rangle_{j k l} . \\
& \bar{\Delta}_{i j k} \Phi_{l}^{i} \varepsilon^{j l m} \equiv(\bar{\Delta} \cdot \Phi)_{k}^{m}, \quad \bar{\Delta}_{i j k} B_{m}^{l} \varepsilon^{k m n} \equiv\lfloor\bar{\Delta} \cdot B\rceil_{i j}^{l n}, \\
& \Delta^{i j m} \Phi_{i}^{l} \varepsilon_{j l k} \equiv(\Phi \cdot \Delta)_{k}^{m},
\end{aligned}
$$

The $(\cdot)$-product of two octets in the above expressions corresponds to the usual matrix multiplication.

\footnotetext{
${ }^{2}$ Another way to look for invariant terms is to write down first all possible tensors build out of one baryon and one meson tensors, and then to combine them to singlets (interchange of $\mathbf{1 0}$ and $\mathbf{8}$ in (C.9)):

$$
\begin{aligned}
{[(\overline{\mathbf{1 0}} \otimes \mathbf{8})} & \otimes(\mathbf{1 0} \otimes \mathbf{8})]_{\text {singlets }} \\
& =[(\mathbf{8} \oplus \overline{\mathbf{1 0}} \oplus \mathbf{2 7} \oplus \overline{\mathbf{3 5}}) \otimes(\mathbf{8} \oplus \mathbf{1 0} \oplus \mathbf{2 7} \oplus \mathbf{3 5})]_{\text {singlets }} \\
& =[\mathbf{8} \otimes \mathbf{8}]_{\text {singlet }} \oplus[\overline{\mathbf{1 0}} \otimes \mathbf{1 0}]_{\text {singlet }} \oplus[\mathbf{2 7} \otimes \mathbf{2 7}]_{\text {singlet }} \oplus[\overline{\mathbf{3 5}} \otimes \mathbf{3 5}]_{\text {singlet }} .
\end{aligned}
$$

These singlet contractions don't lead to any new invariant terms since they can be rewritten with the help of the contractions in (C.9).
} 
From (C.11) four possible $S U(3)$-invariant contractions are obtained:

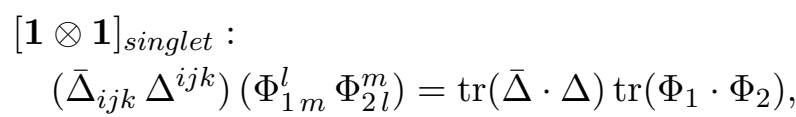

$\left[\mathbf{8} \otimes \mathbf{8}_{S}\right]_{\text {singlet }}:$

$$
\left(\bar{\Delta}_{i j k} \Delta^{i j l}\right)\left(\Phi_{1 m}^{k} \Phi_{2 l}^{m}+\Phi_{2 m}^{k} \Phi_{1 l}^{m}\right)=(\bar{\Delta} \cdot \Delta)_{k}^{l}\left\{\Phi_{1}, \Phi_{2}\right\}_{l}^{k}=\operatorname{tr}\left((\bar{\Delta} \cdot \Delta)\left\{\Phi_{1}, \Phi_{2}\right\}\right),
$$

$\left[\mathbf{8} \otimes \mathbf{8}_{A}\right]_{\text {singlet }}:$

$$
\left(\bar{\Delta}_{i j k} \Delta^{i j l}\right)\left(\Phi_{1 m}^{k} \Phi_{2 l}^{m}-\Phi_{2 m}^{k} \Phi_{1 l}^{m}\right)=(\bar{\Delta} \cdot \Delta)_{k}^{l}\left[\Phi_{1}, \Phi_{2}\right]_{l}^{k}=\operatorname{tr}\left((\bar{\Delta} \cdot \Delta)\left[\Phi_{1}, \Phi_{2}\right]\right),
$$

$[\mathbf{2 7} \otimes \mathbf{2 7}]_{\text {singlet }}:$

$$
\left(\bar{\Delta}_{i j k} \Delta^{i l m}\right)\left(\Phi_{1 l}^{j} \Phi_{2 m}^{k}\right)_{s y m(l m)}^{\operatorname{sym}(j k)}=2 \operatorname{tr}\left(\lfloor\bar{\Delta} \cdot \Delta\rceil\left\lfloor\Phi_{1} \cdot \Phi_{2}\right\rceil\right)+2 \operatorname{tr}\left(\lfloor\bar{\Delta} \cdot \Delta\rceil\left\lfloor\Phi_{2} \cdot \Phi_{1}\right\rceil\right) .
$$

Again, the given contractions here do not always represent pure multiplets. Those would be obtained by additional subtractions. As an example, we mention the contractions of the baryonic tensors. For the $\mathbf{8}$, the singlet component needs to be subtracted:

$$
8-\text { plet }=\bar{\Delta}_{\mathrm{ijk}} \Delta^{\mathrm{ijl}}-\frac{1}{3} \delta_{\mathrm{k}}^{\mathrm{l}} \Delta_{\mathrm{ijm}} \Delta^{\mathrm{ijm}}=(\bar{\Delta} \cdot \Delta)_{\mathrm{k}}^{1}-\frac{1}{3} \delta_{\mathrm{k}}^{\mathrm{l}} \operatorname{tr}(\bar{\Delta} \cdot \Delta) .
$$

Given the totally symmetric tensor $R_{j k}^{l m} \equiv \bar{\Delta}_{i j k} \Delta^{i l m}$, the 27-plet is obtained upon subtraction of the $\mathbf{1}$ and $\mathbf{8}$ in $R_{j k}^{l m}$ :

$$
\begin{aligned}
27-\text { plet } & =R_{j k}^{l m}-\frac{1}{3} \delta_{k}^{m} R_{j o}^{l o}-\frac{1}{9} \delta_{k}^{m} \delta_{j}^{l} R_{o p}^{o p} \\
& =\lfloor\bar{\Delta} \cdot \Delta\rceil_{j k}^{l m}-\frac{1}{3} \delta_{k}^{m}(\bar{\Delta} \cdot \Delta)_{j}^{l}-\frac{1}{9} \delta_{k}^{m} \delta_{j}^{l} \operatorname{tr}(\bar{\Delta} \cdot \Delta) .
\end{aligned}
$$

The contractions in the last line in (C.13) can be rewritten by means of the simple algebraical identity

$$
\begin{aligned}
\operatorname{tr}\left(\left(\bar{\Delta} \cdot \Phi_{1}\right)\left(\Phi_{2} \cdot \Delta\right)\right) & =\bar{\Delta}_{i j k} \Delta^{n o k} \Phi_{1 l}^{i} \Phi_{2 n}^{p}\left(\delta_{o}^{j} \delta_{p}^{l}-\delta_{p}^{j} \delta_{o}^{l}\right) \\
& =\bar{\Delta}_{i j k} \Delta^{n j k} \Phi_{1 p}^{i} \Phi_{2 n}^{p}-\bar{\Delta}_{i j k} \Delta^{n l k} \Phi_{1 l}^{i} \Phi_{2 n}^{j} \\
& =\operatorname{tr}\left((\bar{\Delta} \cdot \Delta)\left(\Phi_{1} \cdot \Phi_{2}\right)\right)-\operatorname{tr}\left(\lfloor\bar{\Delta} \cdot \Delta\rceil\left\lfloor\Phi_{1} \cdot \Phi_{2}\right\rceil\right) .
\end{aligned}
$$

Using this "Fierz-like"-identity we observe, that the flavour-structure in the $S U(3)$ invariant vertex (C.11) can be completely furnished by ${ }^{3}$

$$
\begin{gathered}
\operatorname{tr}(\bar{\Delta} \cdot \Delta) \operatorname{tr}\left(\Phi_{1} \cdot \Phi_{2}\right), \quad \operatorname{tr}\left((\bar{\Delta} \cdot \Delta)\left[\Phi_{1}, \Phi_{2}\right]_{ \pm}\right), \\
\operatorname{tr}\left(\left(\bar{\Delta} \cdot \Phi_{1}\right)\left(\Phi_{2} \cdot \Delta\right)\right)+\operatorname{tr}\left(\left(\bar{\Delta} \cdot \Phi_{2}\right)\left(\Phi_{1} \cdot \Delta\right)\right) .
\end{gathered}
$$

${ }^{3}$ Note, that the obvious antisymmetric version of the last line in (C.18) can be rewritten with the help of the structure already included:

$$
\operatorname{tr}\left(\left(\bar{\Delta} \cdot \Phi_{1}\right)\left(\Phi_{2} \cdot \Delta\right)\right)-\operatorname{tr}\left(\left(\bar{\Delta} \cdot \Phi_{1}\right)\left(\Phi_{2} \cdot \Delta\right)\right)=\operatorname{tr}\left((\bar{\Delta} \cdot \Delta)\left[\Phi_{1}, \Phi_{2}\right]_{-}\right) .
$$


Similar steps have to be performed for the construction of the vertex $\bar{\Delta} B \Phi_{1} \Phi_{2}$. The singlets in the product of these four tensors are given by

$$
\begin{aligned}
{[(\overline{\mathbf{1 0}} \otimes \mathbf{8})} & \otimes(\mathbf{8} \otimes \mathbf{8})]_{\text {singlets }} \\
= & {\left[(\mathbf{8} \oplus \overline{\mathbf{1 0}} \oplus \mathbf{2 7} \oplus \overline{\mathbf{3 5}}) \otimes\left(\mathbf{1} \oplus \mathbf{8}_{S} \oplus \mathbf{8}_{A} \oplus \mathbf{1 0} \oplus \overline{\mathbf{1 0}} \oplus \mathbf{2 7}\right)\right]_{\text {singlets }} } \\
& =\left[\mathbf{8} \otimes \mathbf{8}_{S}\right]_{\text {singlet }} \oplus\left[\mathbf{8} \otimes \mathbf{8}_{A}\right]_{\text {singlet }} \oplus[\overline{\mathbf{1 0}} \otimes \mathbf{1 0}]_{\text {singlet }} \oplus[\mathbf{2 7} \otimes \mathbf{2 7}]_{\text {singlet }} .
\end{aligned}
$$

The invariant flavour-contractions in this case are

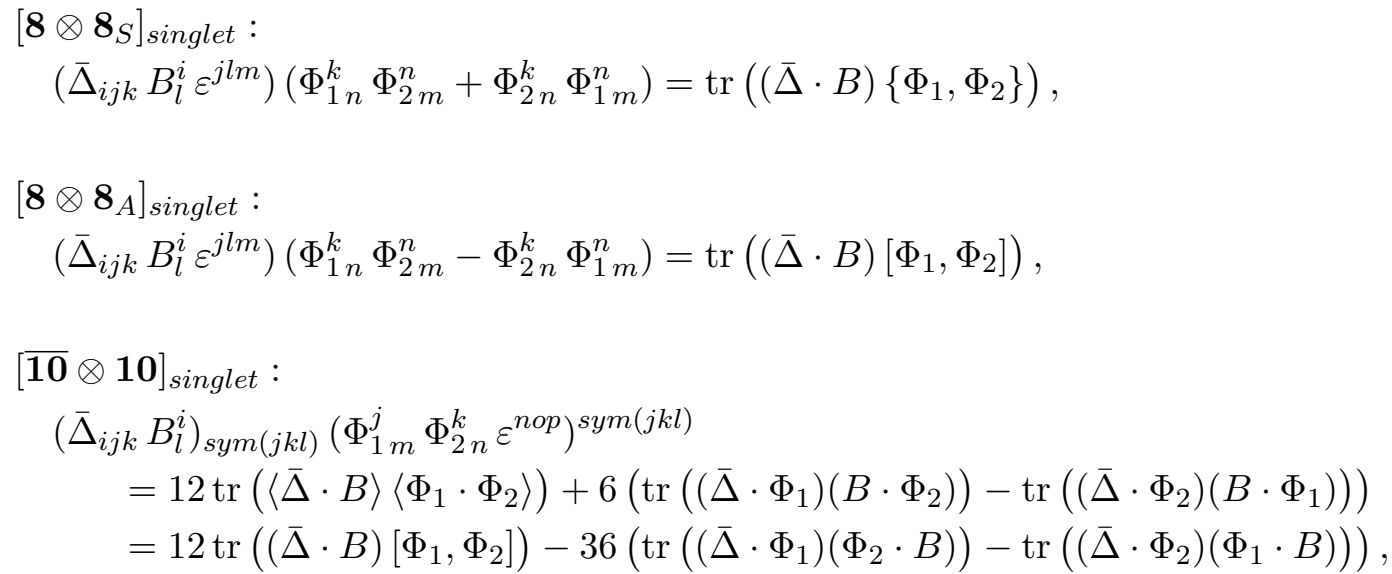

$[\mathbf{2 7} \otimes \mathbf{2 7}]_{\text {singlet }}:$

$$
\begin{aligned}
& \left(\bar{\Delta}_{i j k} B_{m}^{l} \varepsilon^{k m n}\right)^{\operatorname{sym}(l n)}\left(\Phi_{1 l}^{i} \Phi_{2 n}^{j}\right)_{\text {sym(ln) }}^{\operatorname{sym}(i j)} \\
& \quad=4\left(\operatorname{tr}\left(\lfloor\bar{\Delta} \cdot B\rceil\left\lfloor\Phi_{1} \cdot \Phi_{2}\right\rceil\right)+\operatorname{tr}\left(\lfloor\bar{\Delta} \cdot B\rceil\left\lfloor\Phi_{2} \cdot \Phi_{1}\right\rceil\right)\right) .
\end{aligned}
$$

In rewriting the contractions in the $\overline{\mathbf{1 0}} \otimes \mathbf{1 0}$-product, the first step is obvious. The second step is done via Fierz-like relations, which are not so obvious at the first sight:

$$
\begin{aligned}
\operatorname{tr}\left(\langle\bar{\Delta} \cdot B\rangle\left\langle\Phi_{1} \cdot \Phi_{2}\right\rangle\right) & =-\operatorname{tr}\left(\left(\bar{\Delta} \cdot \Phi_{1}\right)\left(B \cdot \Phi_{2}\right)\right)+\operatorname{tr}\left(\left(\bar{\Delta} \cdot \Phi_{2}\right)\left(B \cdot \Phi_{1}\right)\right) \\
\operatorname{tr}\left((\bar{\Delta} \cdot B)\left[\Phi_{1}, \Phi_{2}\right]\right) & =\operatorname{tr}\left(\left(\bar{\Delta} \cdot \Phi_{1}\right)\left(B \cdot \Phi_{2}\right)\right)-\operatorname{tr}\left(\left(\bar{\Delta} \cdot \Phi_{2}\right)\left(B \cdot \Phi_{1}\right)\right) \\
& +2\left(\operatorname{tr}\left(\left(\bar{\Delta} \cdot \Phi_{1}\right)\left(\Phi_{2} \cdot B\right)\right)-\operatorname{tr}\left(\left(\bar{\Delta} \cdot \Phi_{2}\right)\left(\Phi_{1} \cdot B\right)\right)\right) .
\end{aligned}
$$

By using further relations

$$
\begin{aligned}
\operatorname{tr}\left(\left(\bar{\Delta} \cdot \Phi_{2}\right)\left(\Phi_{1} \cdot B\right)\right) & =-\operatorname{tr}\left(\lfloor\bar{\Delta} \cdot B\rceil\left\lfloor\Phi_{1} \cdot \Phi_{2}\right\rceil\right), \\
\operatorname{tr}\left(\left\langle\bar{\Delta} \cdot \Phi_{2}\right\rangle\left\langle\Phi_{1} \cdot B\right\rangle\right) & =-\operatorname{tr}\left(\langle\bar{\Delta} \cdot B\rangle\left\langle\Phi_{1} \cdot \Phi_{2}\right\rangle\right), \\
\operatorname{tr}\left(\left\lfloor\bar{\Delta} \cdot \Phi_{2}\right\rceil\left\lfloor\Phi_{1} \cdot B\right\rceil\right) & =-\operatorname{tr}\left((\bar{\Delta} \cdot B)\left(\Phi_{1} \cdot \Phi_{2}\right)\right),
\end{aligned}
$$

one observes, that the interchange of the octets $B$ and $\Phi$ in (C.20) doesn't lead to any new flavour structures. In this work, the contractions

$$
\operatorname{tr}\left((\bar{\Delta} \cdot B)\left[\Phi_{1}, \Phi_{2}\right]_{ \pm}\right), \quad \operatorname{tr}\left(\left(\bar{\Delta} \cdot \Phi_{1}\right)\left(\Phi_{2} \cdot B\right)\right) \pm \operatorname{tr}\left(\left(\bar{\Delta} \cdot \Phi_{2}\right)\left(\Phi_{1} \cdot B\right)\right)
$$

are chosen to construct the complete basis in the flavour space for the vertex with the flavour structure as in (C.11). 
Construction of the symmetry breaking part of the chiral Lagrangian proceeds along the same path. The parameter of the symmetry breaking, $\chi_{0}$, transforms as $\mathbf{1} \oplus \mathbf{8}$. Terms of the chiral order $Q^{4}$, which are subject of Section 1.4.3, are obtained by building all possible contractions of baryon tensors with two powers of $\chi_{0}$. For the octet baryons the minimal set of independent terms is obtained from the singlets in the product:

$$
\mathbf{8} \otimes \mathbf{8} \otimes(\mathbf{1} \oplus \mathbf{8}) \otimes(\mathbf{1} \oplus \mathbf{8})
$$

It holds:

$$
\begin{gathered}
\operatorname{tr}(\bar{B} B) \operatorname{tr}\left(\chi_{0}^{2}\right), \quad \operatorname{tr}\left(\bar{B}\left[\left[\chi_{0}, \chi_{0}\right]_{+}, B\right]_{ \pm}\right), \quad \operatorname{tr}\left(\bar{B} \chi_{0}\right) \operatorname{tr}\left(\chi_{0} B\right) \\
\operatorname{tr}\left(\bar{B}\left[\chi_{0}, B\right]_{ \pm}\right) \operatorname{tr}\left(\chi_{0}\right), \quad \operatorname{tr}(\bar{B} B)\left(\operatorname{tr}\left(\chi_{0}\right)\right)^{2}
\end{gathered}
$$

Here, the first line corresponds to the contractions in (C.8), where both mesonic octets are replaced by $\chi_{0}$ (only symmetric components survive). The additional contractions in the second line are possible due to the singlet component in $\chi_{0}\left(\operatorname{tr}\left(\chi_{0}\right) \neq 0\right)$. Similar for the decuplet baryons, the singlets in the product

$$
\overline{10} \otimes 10 \otimes(1 \oplus 8) \otimes(1 \oplus 8)
$$

lead to

$$
\begin{gathered}
\operatorname{tr}(\bar{\Delta} \cdot \Delta) \operatorname{tr}\left(\chi_{0}^{2}\right), \quad \operatorname{tr}\left((\bar{\Delta} \cdot \Delta)\left[\chi_{0}, \chi_{0}\right]_{+}\right), \quad \operatorname{tr}\left(\left(\bar{\Delta} \cdot \chi_{0}\right)\left(\chi_{0} \cdot \Delta\right)\right) \\
\operatorname{tr}\left((\bar{\Delta} \cdot \Delta) \chi_{0}\right) \operatorname{tr}\left(\chi_{0}\right) \quad \operatorname{tr}(\bar{\Delta} \cdot \Delta)\left(\operatorname{tr}\left(\chi_{0}\right)\right)^{2}
\end{gathered}
$$

There is another complete set of independent flavour contractions, that is frequently used in the literature (see e.g. $[12,60]$ ) and that partially differs from the set in (C.25). Terms, used in the cited works, which don't explicitly occur in (C.25), are maped onto the basis introduced here as follows:

$$
\begin{aligned}
\operatorname{tr}\left(\bar{B}\left[\chi_{0},\left[\chi_{0}, B\right]_{-}\right]_{-}\right) & =\frac{1}{2} \operatorname{tr}\left(\bar{B}\left[\left[\chi_{0}, \chi_{0}\right]_{+}, B\right]_{+}\right)-2 \operatorname{tr}\left(\bar{B} \chi_{0} B \chi_{0}\right), \\
\operatorname{tr}\left(\bar{B}\left[\chi_{0},\left[\chi_{0}, B\right]_{+}\right]_{-}\right) & =\frac{1}{2} \operatorname{tr}\left(\bar{B}\left[\left[\chi_{0}, \chi_{0}\right]_{+}, B\right]_{-}\right), \\
\operatorname{tr}\left(\bar{B}\left[\chi_{0},\left[\chi_{0}, B\right]_{+}\right]_{+}\right) & =\frac{1}{2} \operatorname{tr}\left(\bar{B}\left[\left[\chi_{0}, \chi_{0}\right]_{+}, B\right]_{+}\right)+2 \operatorname{tr}\left(\bar{B} \chi_{0} B \chi_{0}\right),
\end{aligned}
$$

with

$$
\begin{aligned}
\operatorname{tr}\left(\bar{B} \chi_{0} B \chi_{0}\right) & =-\frac{1}{2} \operatorname{tr}(\bar{B} B)\left(\operatorname{tr}\left(\chi_{0}\right)\right)^{2}+\frac{1}{2} \operatorname{tr}(\bar{B} B) \operatorname{tr}\left(\chi_{0}^{2}\right)+\operatorname{tr}\left(\bar{B}\left[\chi_{0}, B\right]_{+}\right) \operatorname{tr}\left(\chi_{0}\right) \\
& -\frac{1}{2} \operatorname{tr}\left(\bar{B}\left[\left[\chi_{0}, \chi_{0}\right]_{+}, B\right]_{+}\right)+\operatorname{tr}\left(\bar{B} \chi_{0}\right) \operatorname{tr}\left(B \chi_{0}\right)
\end{aligned}
$$




\begin{tabular}{|c||c|c|c|}
\hline$\Gamma$ & $p_{\Gamma}$ & $c_{\Gamma}$ & $h_{\Gamma}$ \\
\hline 1 & 0 & 0 & 0 \\
$\gamma_{5}$ & 1 & 0 & 1 \\
$\gamma_{\mu}$ & 0 & 1 & 0 \\
$\gamma_{5} \gamma_{\mu}$ & 1 & 0 & 0 \\
$\sigma_{\mu \nu}$ & 0 & 1 & 0 \\
\hline
\end{tabular}

Table C.1.: Transformation properties of the elements of the Clifford-algebra.

\section{C.2. Charge conjugation}

In this section the transformation properties of field operators under charge conjugation are discussed. The discussion is extended to objects carrying $S U(3)$ flavour indices. The main focus is put on the four-point interaction introduced in Section 1.4.2.

A scalar field is transformed under charge conjugation, up to a phase $\xi$ with $\xi^{2}=1$, to its complex conjugated partner:

$$
\phi \stackrel{C}{\rightarrow} \phi^{c}=\xi \phi^{*}
$$

In addition to the complex conjugation, charge conjugation of fermions takes care of the spinor structure via

$$
\psi^{c}=C \gamma_{0} \psi^{*}=C \bar{\psi}^{T}, \quad \bar{\psi}_{c}=\left(\psi^{\dagger} \gamma^{0}\right)^{c}=\psi^{T} C,
$$

with the charge conjugation matrix in the Dirac representation

$$
C=i \gamma^{2} \gamma^{0}=-C^{-1}=-C^{\dagger}=-C^{T}, \quad C C^{\dagger}=1, C^{2}=-1 .
$$

Fermion bilinears, constructed using an arbitrary element of the Clifford-algebra

$$
\Gamma \in 1, \gamma_{5}, \gamma^{\mu}, \gamma_{5} \gamma^{\mu}, \sigma^{\mu \nu}
$$

transform as follows:

$$
\begin{aligned}
\bar{\psi} \Gamma \psi \stackrel{\mathcal{C}}{\rightarrow} \psi^{T}(C \Gamma C) \bar{\psi}^{T} & =-\bar{\psi}(C \Gamma C)^{T} \psi=-\bar{\psi} C^{T} \Gamma^{T} C^{T} \psi=\bar{\psi} C \Gamma^{T} C^{-1} \psi \\
& =(-1)^{c_{\Gamma}} \bar{\psi} \Gamma \psi .
\end{aligned}
$$

The change of the sign in the last step is due to the interchange of the fermion fields. Furthermore, the coefficients $c_{\Gamma}$ are defined via

$$
C \Gamma C^{-1}=(-1)^{c_{\Gamma}} \Gamma^{T}, \quad(-1)^{c_{\Gamma}} \Gamma=C \Gamma C^{-1} .
$$

To distinguish the different transformation behaviour of vectors building the basis of the 
fundamental and conjugated representations of $S U(N)$, one introduces contra- and covariant indices, respectively. In the $S U(3)$ flavour symmetry group of strong interactions, the contra- and covariant indices label the different flavours of quarks and antiquarks, respectively. Charge conjugation interchanges particles and antiparticles. Therefore, the generalisation of this operation to $S U(3)$-tensors is obtained by the interchange of the co- and contravariant indices. For the mesonic octet one has:

$$
\Phi_{j}^{i} \stackrel{c . c .}{\rightarrow} \Phi_{i}^{j}=\left(\Phi^{T}\right)_{j}^{i}
$$

which one immediately expects by looking at the explicit form of the matrix $\Phi$ in (1.24). By expressing the meson matrix $\Phi$ as a function of neutral scalar fields with $\phi^{c}=\xi \phi$

$$
\Phi_{j}^{i}=\sum_{a}\left(\lambda^{(a)}\right)_{j}^{i} \phi_{a} \stackrel{\mathcal{C}}{\rightarrow} \sum_{a}\left(\lambda^{(a)}\right)_{j}^{i} \xi_{a} \phi^{a *}=\sum_{a}\left(\lambda^{(a)}\right)_{j}^{i} \xi_{a} \phi^{a}
$$

we note, that the charge conjugation parities $\xi_{a}$ have to be chosen in an appropriate way as to guarantee ${ }^{4}$

$$
\lambda^{(a)} \xi_{a} \stackrel{!}{=} \lambda^{(a)^{T}} .
$$

Similar for the components of the baryon matrix $B$ :

$$
B_{\alpha j}^{i} \stackrel{\mathcal{C}}{\rightarrow} C_{\alpha \beta} \bar{B}_{\beta i}^{j}, \quad \bar{B}_{\alpha j}^{i} \stackrel{\mathcal{C}}{\rightarrow} B_{\beta i}^{j} C_{\beta \alpha},
$$

or in the (flavour-)matrix form:

$$
B_{\alpha} \stackrel{\mathcal{C}}{\rightarrow} C_{\alpha \beta} \bar{B}_{\beta}^{T}, \quad \text { and } \quad \bar{B}_{\alpha} \stackrel{\mathcal{C}}{\rightarrow} B_{\beta}^{T} C_{\beta \alpha} .
$$

By writting out the spinor-indices (greek letters) explicitly, the tranposition $T$ in the above relation takes care of the flavour indices only. The transformation of the spinors structure of $B_{j}^{i}$ is done as for the single fermionic field in (C.29).

Similar, the transformation of (anti-)decuplet tensor-fields is given by ${ }^{5}$ :

$$
\Delta_{\alpha}^{i j k} \stackrel{\mathcal{C}}{\rightarrow} C_{\alpha \beta} \bar{\Delta}_{\beta i j k}, \quad \text { and } \quad \bar{\Delta}_{\alpha i j k} \stackrel{\mathcal{C}}{\rightarrow} \Delta_{\beta}^{i j k} C_{\beta \alpha} .
$$

We check the transformation behaviour of some building blocks which are used in the course of the construction of the four-point chiral interaction. Adopting the compact notation of [57], we denote in this section the commutator and the anticommutator of two mesonic octets by $\left(\Phi_{1}, \Phi_{2}\right)$. Transformation of this object under charge conjugation is given by

$$
\left(\Phi_{1}, \Phi_{2}\right) \stackrel{\mathcal{C}}{\rightarrow}(-1)^{c_{\Phi}}\left(\Phi_{1}, \Phi_{2}\right)^{T}, \quad \text { with } \quad c_{\Phi} \equiv \begin{cases}+1 & \text { if anticommutator }, \\ -1 & \text { if commutator. }\end{cases}
$$

\footnotetext{
${ }^{4}$ Actually, constructing the matrix $\Phi$ in the form as shown in (1.24), the correspondence of the Cartesian to the physical fields and the choose of phases is done in such a way, that $\Phi \stackrel{\text { c.c. }}{\rightarrow} \Phi^{T}$, is valid. See e.g. [33].

${ }^{5}$ Lorentz-indices of the spin-3/2 fields are omitted troughout this section.
} 
An octet, built out of a baryonic anti-decuplet and a mesonic octet, transforms $\mathrm{as}^{6}$

$$
\left(\bar{\Delta}_{\alpha} \cdot \Phi\right)_{k}^{m}=\bar{\Delta}_{\alpha i j k} \Phi_{l}^{i} \varepsilon^{j l m} \stackrel{\mathcal{C}}{\rightarrow} \Delta_{\rho}^{i j k} C_{\rho \alpha} \Phi_{i}^{l} \varepsilon_{j l m}=\left(\Phi \cdot \Delta_{\rho}\right)_{m}^{k} C_{\rho \alpha}
$$

or in the matrix notation:

$$
\left(\bar{\Delta}_{\alpha} \cdot \Phi\right) \stackrel{\mathcal{C}}{\rightarrow}\left(\Phi \cdot \Delta_{\rho}\right)^{T} C_{\rho \alpha}
$$

Similar, combinations of the (anti-)decuplet tensor with a baryonic octet transform as

$$
(\bar{\Delta} \cdot \Gamma B) \stackrel{\mathcal{C}}{\rightarrow}(-1)^{c_{\Gamma}}(\bar{B} \cdot \Gamma \Delta)^{T}, \quad \text { and } \quad(\bar{B} \cdot \Gamma \Delta) \stackrel{\mathcal{C}}{\rightarrow}(-1)^{c_{\Gamma}}(\bar{\Delta} \cdot \Gamma B)^{T}
$$

Having stated the transformation properties of the building blocks, several $S U(3)$ invariants, which are used to set up the flavour basis in (C.8), (C.18) and in (C.23), can be checked now. Invariants in the product of four octets transform as ${ }^{7}$ :

$$
\begin{array}{rll}
\operatorname{tr}(\bar{B} \Gamma B) \operatorname{tr}\left(\Phi_{1} \Phi_{2}\right) & \stackrel{\mathcal{C}}{\rightarrow} & (-1)^{c_{\Gamma}} \operatorname{tr}(\bar{B} \Gamma B) \operatorname{tr}\left(\Phi_{1} \Phi_{2}\right), \\
\operatorname{tr}\left(\bar{B} \Phi_{1}\right) \Gamma \operatorname{tr}\left(\Phi_{2} B\right) & \stackrel{\mathcal{C}}{\rightarrow} & (-1)^{c_{\Gamma}} \operatorname{tr}\left(\bar{B} \Phi_{2}\right) \Gamma \operatorname{tr}\left(\Phi_{1} B\right) \\
\operatorname{tr}\left(\bar{B}\left[\left(\Phi_{1}, \Phi_{2}\right), B\right]_{ \pm}\right) & \stackrel{\mathcal{C}}{\rightarrow} & (-1)^{c_{\Gamma}+c_{\Phi}} \operatorname{tr}\left(\bar{B}\left[\left(\Phi_{1}, \Phi_{2}\right), B\right]_{ \pm}\right) \\
\operatorname{tr}\left(\bar{B} \Phi_{1} \Gamma B \Phi_{2}\right) & \stackrel{\mathcal{C}}{\rightarrow} & (-1)^{c_{\Gamma}} \operatorname{tr}\left(\bar{B} \Phi_{1} \Gamma B \Phi_{2}\right)
\end{array}
$$

Charge conjugation of invariants in the $\overline{\mathbf{1 0}} \otimes \mathbf{1 0} \otimes \mathbf{8} \otimes \mathbf{8}$-vertex leads to

$$
\begin{array}{rll}
\operatorname{tr}\left(\bar{\Delta}_{1} \cdot \Gamma \Delta_{2}\right) \operatorname{tr}\left(\Phi_{1} \Phi_{2}\right) & \stackrel{\mathcal{C}}{\rightarrow}(-1)^{c_{\Gamma}} \operatorname{tr}\left(\bar{\Delta}_{2} \cdot \Gamma \Delta_{1}\right) \operatorname{tr}\left(\Phi_{1} \Phi_{2}\right), \\
\operatorname{tr}\left(\left(\bar{\Delta}_{1} \cdot \Phi_{1}\right) \Gamma\left(\Phi_{2} \cdot \Delta_{2}\right)\right) & \stackrel{\mathcal{C}}{\rightarrow}(-1)^{c_{\Gamma}} \operatorname{tr}\left(\left(\bar{\Delta}_{2} \cdot \Phi_{2}\right) \Gamma\left(\Phi_{1} \cdot \Delta_{1}\right)\right), \\
\operatorname{tr}\left(\left(\bar{\Delta}_{1} \cdot \Gamma \Delta_{2}\right)\left(\Phi_{1}, \Phi_{2}\right)\right) & \stackrel{\mathcal{C}}{\rightarrow}(-1)^{\left(c_{\Gamma}+c_{\Phi}\right)} \operatorname{tr}\left(\left(\bar{\Delta}_{2} \cdot \Gamma \Delta_{1}\right)\left(\Phi_{1}, \Phi_{2}\right)\right) .
\end{array}
$$

Finally, for the invariants in the $\overline{\mathbf{1 0}} \otimes \mathbf{8} \otimes \mathbf{8} \otimes \mathbf{8}$-vertex it holds:

$$
\begin{array}{rll}
\operatorname{tr}\left((\bar{\Delta} \cdot \Gamma B)\left(\Phi_{1}, \Phi_{2}\right)\right) & \stackrel{\mathcal{C}}{\rightarrow}(-1)^{\left(c_{\Gamma}+c_{\Phi}\right)} \operatorname{tr}\left((\bar{B} \cdot \Gamma \Delta)\left(\Phi_{1}, \Phi_{2}\right)\right), \\
\operatorname{tr}\left(\left(\bar{\Delta} \cdot \Phi_{1}\right) \Gamma\left(\Phi_{2} \cdot B\right)\right) & \stackrel{\mathcal{C}}{\rightarrow}(-1)^{\left(c_{\Gamma}\right)} \operatorname{tr}\left(\left(\bar{B} \cdot \Phi_{2}\right) \Gamma\left(\Phi_{1} \cdot \Delta\right)\right) .
\end{array}
$$

\footnotetext{
${ }^{6}$ In raising and lowering flavour indices, the identity $\varepsilon^{i j k}=\varepsilon_{i j k}$ is used in order to achieve covariant results.

${ }^{7}$ Straightforward generalisation to the arbitrary number of meson octets in the relations (C.44 - C.45) is carried out in [57]. Transformation properties of the last antisymmetric flavour contractions in (C.8), $\operatorname{tr}\left(\bar{B} \Phi_{1} \Gamma B \Phi_{2}\right)-\operatorname{tr}\left(\bar{B} \Phi_{2} \Gamma B \Phi_{1}\right)$, have to be studied separately with the help of (C.46).
} 


\section{Appendix D.}

\section{Baryon states}

Construction of hadron states with given flavour and spin content is treated in many textbooks (see e.g. [59, 44]). In this chapter the construction of baryon states using tensor methods is recalled for the number of flavours $N_{F}=3$.

For $N_{c}=3$, the baryon states are constructed in the Fock space defined by the action of three quark creation operators on the vacuum state. The colour structure of baryons, being colour singlets, is obtained by contracting the colour indices of the quark operators with the $\varepsilon$-tensor of $S U(3)$. This makes the colour part of the baryon wave function antisymmetric under interchange of two quarks. The antisymmetry of the total wave function of fermions requires the spin-flavour part to be totally symmetric under interchange of two quarks. We discuss the flavour and spin parts separately and give at the end of this chapter the explicite expressions of the flavour $S U(3)$ octet and decuplet baryon states.

\section{D.1. Flavour part}

Quarks/antiquarks are identified with the orthonormal basis of the support of the fundamental/conjugate representation of $S U(3)$. The "fundamental triplet" $\mathbf{3}$ is

$$
q^{i}=\left(\begin{array}{c}
q^{1} \\
q^{2} \\
q^{3}
\end{array}\right) .
$$

The components of the vector $q^{i}$ are identified with the different flavours of the three lightest quarks. The up, down and strange quarks are

$$
q^{i}=u, \quad q^{2}=d, \quad q^{3}=s .
$$

Similar, the antiquarks correspond to the anti-triplet $\overline{\mathbf{3}}$ :

$$
q_{i}=\left(q_{1}, q_{2}, q_{3}\right)=(\bar{u}, \bar{d}, \bar{s}) .
$$

Concerning the flavour structure of the baryons, the decomposition of the product of three quarks is

$$
(\mathbf{3} \otimes \mathbf{3}) \otimes \mathbf{3}=(\mathbf{6} \oplus \overline{\mathbf{3}}) \otimes \mathbf{3}=(\mathbf{6} \otimes \mathbf{3}) \oplus(\overline{\mathbf{3}} \otimes \mathbf{3})=(\mathbf{1 0} \oplus \mathbf{8}) \oplus(\mathbf{1} \oplus \mathbf{8}) .
$$


The product $\mathbf{3} \otimes \mathbf{3}$ is decomposed as follows:

$$
\begin{aligned}
q^{i} q^{j} & =\frac{1}{2}\left(q^{i} q^{j}+q^{j} q^{i}\right)+\frac{1}{2}\left(q^{i} q^{j}-q^{j} q^{i}\right)=\frac{1}{2}\left(q^{i} q^{j}+q^{j} q^{i}\right)+\frac{1}{2} \varepsilon^{i j k} \varepsilon_{k l m} q^{l} q^{m} \\
& =\underbrace{q^{i j}}_{\mathbf{6}}+\frac{1}{2} \varepsilon^{i j k} \underbrace{\varepsilon_{k l m} q^{l} q^{m}}_{\overline{\mathbf{3}}} .
\end{aligned}
$$

with

$$
q^{i j} \equiv \frac{1}{2}\left(q^{i} q^{j}+q^{j} q^{i}\right)
$$

We decompose further $\mathbf{6} \otimes \mathbf{3}$

$$
\begin{aligned}
q^{i j} q^{k} & =\frac{1}{3}\left(q^{i j} q^{k}+q^{i k} q^{j}+q^{k j} q^{i}\right)+\frac{1}{3}\left(q^{i j} q^{k}-q^{k j} q^{i}\right)+\frac{1}{3}\left(q^{i j} q^{k}-q^{i k} q^{j}\right) \\
& =\frac{1}{3} \underbrace{\left(q^{i j} q^{k}+q^{i k} q^{j}+q^{k j} q^{i}\right)}_{\mathbf{1 0}}+\frac{1}{3} \underbrace{\left(\varepsilon^{i k l} \varepsilon_{n o p} q^{m j} q^{n}+\varepsilon^{j k l} \varepsilon_{n o p} q^{i m} q^{n}\right)}_{\mathbf{8}},
\end{aligned}
$$

and $\overline{3} \otimes 3$

$$
q_{j} q^{i}=\underbrace{\left(q_{j} q^{i}-\frac{1}{3} \delta_{j}^{i} q_{k} q^{k}\right)}_{\mathbf{8}}+\underbrace{\frac{1}{3} \delta_{j}^{i} q_{k} q^{k}}_{\mathbf{1}} .
$$

The above relation for the anti-triplet in (D.5) is:

$$
\varepsilon_{j l m} q^{l} q^{m} q^{i}=\underbrace{\left(\varepsilon_{j l m} q^{l} q^{m} q^{i}-\frac{1}{3} \delta_{j}^{i} \varepsilon_{k l m} q^{l} q^{m} q^{k}\right)}_{\mathbf{8}}+\underbrace{\frac{1}{3} \delta_{j}^{i} \varepsilon_{k l m} q^{l} q^{m} q^{k}}_{\mathbf{1}} .
$$

The eight observed spin-1/2 baryons are recognised as building an octet, given by the traceless tensor $B_{j}^{i}$ :

$$
B_{j}^{i} \equiv \varepsilon_{j l m} q^{l} q^{m} q^{i}-\frac{1}{3} \delta_{j}^{i} \varepsilon_{k l m} q^{l} q^{m} q^{k}
$$

or by the tensor $\tilde{B}_{j}^{i}$, defined as

$$
\tilde{B}_{j}^{i} \equiv \varepsilon_{j l m} q^{i l} q^{m}
$$

Octet baryons can be labeled either by two indices in the fundamental representation $(i, j=1,2,3)$ of $S U(3)$ or by a single adjoint index $a(a=1, \ldots, 8)$ :

$$
B_{j}^{i}=\sum_{a=1}^{8} b_{a}\left(\lambda^{(a)}\right)_{j}^{i}, \quad \tilde{B}_{j}^{i}=\sum_{a=1}^{8} \tilde{b}_{a}\left(\lambda^{(a)}\right)_{j}^{i},
$$


$\frac{1}{2} \lambda^{(a)}$ being the generators of the flavour symmetry group. Main properties of the GellMann-matrices are given at the end of this appendix. Tensors $b_{a}$ are given by

$$
\begin{aligned}
b_{a} & =\frac{1}{2} \operatorname{tr}\left(B \lambda^{(a)}\right)=\frac{1}{2} B_{j}^{i}\left(\lambda^{(a)}\right)_{i}^{j}=\frac{1}{2}\left(\lambda^{(a)}\right)_{i}^{j}\left(\varepsilon_{j l m} q^{l} q^{m} q^{i}-\frac{1}{3} \delta_{j}^{i} \varepsilon_{k l m} q^{l} q^{m} q^{k}\right) \\
& \stackrel{\lambda_{i}^{i}=0}{=}\left(\lambda^{(a)}\right)_{i}^{j} \varepsilon_{j l m} q^{l} q^{m} q^{i} .
\end{aligned}
$$

Similar:

$$
\tilde{b}_{a}=\frac{1}{2} \operatorname{tr}\left(\tilde{B} \lambda^{(a)}\right)=\left(\lambda^{(a)}\right)_{i}^{j} \varepsilon_{j l m} q^{i l} q^{m} .
$$

By comparing the isospin and hypercharge properties of the observed baryons with those of quarks, the components of $B$-matrix are identified as follows (see e.g. [59]):

$$
B=\left(\begin{array}{ccc}
\frac{1}{\sqrt{2}} \Sigma^{0}+\frac{1}{\sqrt{6}} \Lambda & \Sigma^{+} & p \\
\Sigma^{-} & -\frac{1}{\sqrt{2}} \Sigma^{0}+\frac{1}{\sqrt{6}} \Lambda & n \\
-\Xi^{-} & \Xi^{0} & -\frac{2}{\sqrt{6}} \Lambda
\end{array}\right) .
$$

The relations of the physical baryons to the tensors $b_{a}$ and $\tilde{b}_{a}$ are given in Table D.1 and in Table D.2, respectively.

Ten observed spin-3/2 baryons are identified as building the $S U(3)$ decuplet, 10 in (D.4), represented by the totaly symmetric tensor $D^{i j k}$. Tensor components of $D^{i j k}$ are identified as $([59])$ :

$$
\begin{gathered}
\Delta^{++}=D^{111}, \quad \Delta^{+}=\frac{1}{\sqrt{3}}\left(D^{112}+D^{121}+D^{211}\right) \\
\Delta^{0}=\frac{1}{\sqrt{3}}\left(D^{122}+D^{212}+D^{221}\right), \quad \Delta^{-}=D^{222} \\
\Sigma^{+}=\frac{1}{\sqrt{3}}\left(D^{113}+D^{131}+D^{311}\right), \quad \Sigma^{-}=\frac{1}{\sqrt{3}}\left(D^{223}+D^{232}+D^{322}\right), \\
\Sigma^{0}=\frac{1}{\sqrt{6}}\left(D^{123}+D^{132}+D^{213}+D^{231}+D^{312}+D^{321}\right), \\
\Xi^{0}=\frac{1}{\sqrt{3}}\left(D^{133}+D^{313}+D^{331}\right), \quad \Xi^{-}=\frac{1}{\sqrt{3}}\left(D^{233}+D^{323}+D^{332}\right), \\
\Omega=D^{333} .
\end{gathered}
$$

Another usefull representation of the decuplet is

$$
\begin{gathered}
D^{111}=\Delta^{++}, \quad D^{112}=\Delta^{+} / \sqrt{3}, \quad D^{122}=\Delta^{0} / \sqrt{3}, \quad D^{222}=\Delta^{-}, \\
D^{113}=\Sigma^{+} / \sqrt{3}, \quad D^{123}=\Sigma^{0} / \sqrt{6}, \quad D^{223}=\Sigma^{-} / \sqrt{3} \\
D^{133}=\Xi^{0} / \sqrt{3}, \quad D^{233}=\Xi^{-} / \sqrt{3} \\
D^{333}=\Omega^{-} .
\end{gathered}
$$

This follows directly from (D.16) because of the total symmetry of $D^{i j k}$. 


\section{D.2. Spin part}

We turn to the spin structure. Three spin-1/2 quarks can be combined to a baryon either with a total spin $1 / 2$ or $3 / 2$. Tensor product of three spin-doublets is

$$
(\mathbf{2} \otimes \mathbf{2}) \otimes \mathbf{2}=(\mathbf{3} \oplus \mathbf{1}) \oplus \mathbf{2}=\mathbf{4} \oplus \mathbf{2} \oplus \mathbf{2} .
$$

One possibility to build a spin- $1 / 2$ wave function for three particles is to combine two of them to a total spin 0 (singlet in $\mathbf{3} \oplus \mathbf{1}$ ) and then to couple the last one to the total spin $1 / 2$. Another possibility is given by a combination, where the first two particles are coupled to spin 1 (triplet in $\mathbf{3} \oplus \mathbf{1}$ ) and then to spin $1 / 2$ by adding the $3 r d$ particle. These two possibilities correspond to the two 2's in (D.18). Four components of a spin-3/2 state correspond to 4 in (D.18).

Coupling of three particles to a given spin can be written down using tensor methods in a similar way as it was done for the flavour part. Notation, that is more suitable for the later evaluation of matrix elements, is achieved with the help of Pauli-matrices

$$
\sigma^{(1)}=\left(\begin{array}{ll}
0 & 1 \\
1 & 0
\end{array}\right), \quad \sigma^{(2)}=\left(\begin{array}{cc}
0 & -i \\
i & 0
\end{array}\right), \quad \sigma^{(3)}=\left(\begin{array}{cc}
1 & 0 \\
0 & -1
\end{array}\right)
$$

and "spin-transition" matrices

$$
S^{(1)}=\left(\begin{array}{cc}
-\frac{1}{\sqrt{2}} & 0 \\
0 & -\frac{1}{\sqrt{6}} \\
\frac{1}{\sqrt{6}} & 0 \\
0 & \frac{1}{\sqrt{2}}
\end{array}\right), \quad S^{(2)}=\left(\begin{array}{cc}
\frac{i}{\sqrt{2}} & 0 \\
0 & \frac{i}{\sqrt{6}} \\
\frac{i}{\sqrt{6}} & 0 \\
0 & \frac{i}{\sqrt{2}}
\end{array}\right), \quad S^{(3)}=\left(\begin{array}{cc}
0 & 0 \\
\sqrt{\frac{2}{3}} & 0 \\
0 & \sqrt{\frac{2}{3}} \\
0 & 0
\end{array}\right) .
$$

The coupling of three doublets $q^{\alpha}(\alpha=1,2)$ to a total spin 1 is achieved by the following combinations:

$$
i \sigma_{\alpha \beta}^{(2)} \delta_{\gamma \chi} q^{\alpha} q^{\beta} q^{\gamma}, \quad-\left(i \sigma^{(2)} \vec{\sigma}\right)_{\alpha \beta} \vec{\sigma}_{\chi \gamma} q^{\alpha} q^{\beta} q^{\gamma}
$$

Here, $\chi$ labels the different spin-components $(\chi=1,2)$. These two combinations correspond to the coupling of first two doublets to an intermediate spin 0 or 1 , respectively. The $\chi$-component of a spin- $3 / 2$ state $(\chi=1, \ldots 4)$ is given by

$$
-\left(i \sigma^{(2)} \vec{\sigma}\right)_{\alpha \beta} \vec{S}_{\chi \gamma} q^{\alpha} q^{\beta} q^{\gamma}
$$

The products of $\vec{\sigma}$ and $\vec{S}$ are defined as

$$
\vec{\sigma} \vec{\sigma}=\sum_{k=1}^{3} \sigma^{(k)} \sigma^{(k)}, \quad \vec{\sigma} \vec{S}=\sum_{k=1}^{3} \sigma^{(k)} S^{(k)}
$$


Denoting $q^{1}$ and $q^{2}$ by $|\uparrow\rangle$ and $|\downarrow\rangle$, the different tensor products of spin-doublets can be given in a more familiar form:

$$
\begin{aligned}
|1 / 2,1 / 2\rangle & \equiv i \sigma_{\alpha \beta}^{(2)} \delta_{\gamma 1} q^{\alpha} q^{\beta} q^{\gamma}=3(|\uparrow \downarrow \uparrow\rangle-|\downarrow \uparrow \uparrow\rangle), \\
|1 / 2,-1 / 2\rangle & \equiv i \sigma_{\alpha \beta}^{(2)} \delta_{\gamma 2} q^{\alpha} q^{\beta} q^{\gamma}=3(|\uparrow \downarrow \downarrow\rangle-|\downarrow \uparrow \downarrow\rangle), \\
|1 / 2,1 / 2\rangle & \equiv-\left(i \sigma^{(2)} \vec{\sigma}\right)_{\alpha \beta} \vec{\sigma}_{1 \gamma} q^{\alpha} q^{\beta} q^{\gamma}=|\downarrow \uparrow \uparrow\rangle+|\uparrow \downarrow \uparrow\rangle+2|\uparrow \uparrow \downarrow\rangle, \\
|1 / 2,-1 / 2\rangle & \equiv-\left(i \sigma^{(2)} \vec{\sigma}\right)_{\alpha \beta} \vec{\sigma}_{2 \gamma} q^{\alpha} q^{\beta} q^{\gamma}=2|\downarrow \downarrow \uparrow\rangle-|\downarrow \uparrow \downarrow\rangle-|\uparrow \downarrow \downarrow\rangle, \\
|3 / 2,3 / 2\rangle & \equiv-\left(i \sigma^{(2)} \vec{\sigma}\right)_{\alpha \beta} \vec{S}_{1 \gamma} q^{\alpha} q^{\beta} q^{\gamma}=\sqrt{2}|\uparrow \uparrow \uparrow\rangle, \\
|3 / 2,1 / 2\rangle & \equiv-\left(i \sigma^{(2)} \vec{\sigma}\right)_{\alpha \beta} \vec{S}_{2 \gamma} q^{\alpha} q^{\beta} q^{\gamma}=\sqrt{2 / 3}(|\downarrow \uparrow \uparrow\rangle+|\uparrow \downarrow \uparrow\rangle+|\uparrow \uparrow \downarrow\rangle), \\
|3 / 2,-1 / 2\rangle & \equiv-\left(i \sigma^{(2)} \vec{\sigma}\right)_{\alpha \beta} \vec{S}_{3 \gamma} q^{\alpha} q^{\beta} q^{\gamma}=\sqrt{2 / 3}(|\downarrow \downarrow \uparrow\rangle+|\downarrow \uparrow \downarrow\rangle+|\uparrow \downarrow \downarrow\rangle), \\
|3 / 2,-3 / 2\rangle & \equiv-\left(i \sigma^{(2)} \vec{\sigma}\right)_{\alpha \beta} \vec{S}_{4 \gamma} q^{\alpha} q^{\beta} q^{\gamma}=\sqrt{2}|\downarrow \downarrow \downarrow\rangle .
\end{aligned}
$$

The states for the flavour octet are obtained either by taking the product of $B_{j}^{i}$ and the first relation in (D.21), or the product of $\tilde{B}_{j}^{i}$ and the second possible spin- $1 / 2$ combination in (D.21). The spin-flavour states for the $S U(3)$ decuplet are constructed by taking a tensor product of the $D^{i j k}$ and (D.22).

$$
\begin{aligned}
|a, \chi\rangle & \left.=\mathcal{N}_{8} \varepsilon_{A B C} \mathcal{B}_{\alpha a \beta b \gamma c}^{(a, \chi)} q_{\alpha a A}^{\dagger} q_{\beta b B}^{\dagger} q_{\gamma c C}^{\dagger} \mid 0\right), \\
\widetilde{|a, \chi\rangle} & \left.=\widetilde{\mathcal{N}}_{8} \varepsilon_{A B C} \widetilde{\mathcal{B}}_{\alpha a \beta b \gamma c}^{(a, \chi)} q_{\alpha a A}^{\dagger} q_{\beta b B}^{\dagger} q_{\gamma c C}^{\dagger} \mid 0\right), \\
|i j k, \chi\rangle & \left.=\mathcal{N}_{10} \varepsilon_{A B C} \mathcal{D}_{\alpha \beta \gamma}^{(\chi)}\left(\delta_{a i} \delta_{b j} \delta_{c k}\right)_{\operatorname{sym}(i j k)} q_{\alpha a A}^{\dagger} q_{\beta b B}^{\dagger} q_{\gamma c C}^{\dagger} \mid 0\right),
\end{aligned}
$$

with

$$
\begin{aligned}
\mathcal{B}_{\alpha a \beta b \gamma c}^{(a, \chi)} & \equiv \varepsilon_{a b g} \lambda_{c g}^{(a)} i \sigma_{\alpha \beta}^{(2)} \delta_{\gamma \chi}, \\
\widetilde{\mathcal{B}}_{\alpha a \beta b \gamma c}^{(a, \chi)} & \equiv\left(\varepsilon_{a c g} \lambda_{b g}^{(a)}+\varepsilon_{b c g} \lambda_{a g}^{(a)}\right)\left(\vec{\sigma} i \sigma^{(2)}\right)_{\alpha \beta} \vec{\sigma}_{\gamma \chi}, \\
\mathcal{D}_{\alpha \beta \gamma}^{(\chi)} & \equiv\left(\vec{\sigma} i \sigma^{(2)}\right)_{\alpha \beta} \vec{S}_{\gamma \chi}^{\dagger}+\left(\vec{\sigma} i \sigma^{(2)}\right)_{\alpha \gamma} \vec{S}_{\beta \chi}^{\dagger}+\left(\vec{\sigma} i \sigma^{(2)}\right)_{\beta \gamma} \vec{S}_{\alpha \chi}^{\dagger}
\end{aligned}
$$

and

$$
\operatorname{sym}(i j k) \equiv \frac{1}{6}(i j k+i k j+j i k+j k i+k i j+k j i) .
$$

The constants $\mathcal{N}_{8}, \widetilde{\mathcal{N}}_{8}$ and $\mathcal{N}_{10}$ guarantee the proper normalisation of the states. Besides the total symmetry of $\mathcal{D}_{\alpha \beta \gamma}^{(\chi)}$, we note the symmetry properties of $\mathcal{B}$ and $\widetilde{\mathcal{B}}$ :

$$
\begin{gathered}
\mathcal{B}_{\alpha a \beta b \gamma c}=-\mathcal{B}_{\alpha b \beta a \gamma c}=-\mathcal{B}_{\beta a \alpha b \gamma c}=\mathcal{B}_{\beta b \alpha a \gamma c}, \\
\widetilde{\mathcal{B}}_{\alpha a \beta b \gamma c}=\widetilde{\mathcal{B}}_{\alpha b \beta a \gamma c}=\widetilde{\mathcal{B}}_{\beta a \alpha b \gamma c}=\widetilde{\mathcal{B}}_{\beta b \alpha a \gamma c} .
\end{gathered}
$$




\begin{tabular}{|c||c|c|}
\hline$\Sigma^{+}$ & $b_{1}-i b_{2}$ & $2 s u u-2 u s u$ \\
$\Sigma^{-}$ & $b_{1}+i b_{2}$ & $2 d s d-2 s d d$ \\
$\Sigma^{-}$ & $b_{3}$ & $d s u-s d u-s u d+u s d$ \\
$p$ & $b_{4}-i b_{5}$ & $2 u d u-2 d u u$ \\
$\Xi^{-}$ & $b_{4}+i b_{5}$ & $2 d s s-2 s d s$ \\
$n$ & $b_{6}-i b_{7}$ & $2 u d d-2 d u d$ \\
$\Xi^{0}$ & $b_{6}+i b_{7}$ & $2 s u s-2 u s s$ \\
$\Lambda$ & $b_{8}$ & $\frac{1}{\sqrt{3}}(d s u+2 d u s-s d u+s u d-2 u d s-u s d)$ \\
\hline
\end{tabular}

Table D.1.: Relation of the octet baryons to the components of $B=b_{a} \lambda_{a}$.

\begin{tabular}{|c||c|c|}
\hline$\Sigma^{+}$ & $\tilde{b}_{1}-i \tilde{b}_{2}$ & $s u u+u s u-2 u u s$ \\
$\Sigma^{-}$ & $\tilde{b}_{1}+i \tilde{b}_{2}$ & $2 d d s-d s d-s d d$ \\
$\Sigma^{-}$ & $\tilde{b}_{3}$ & $\frac{1}{2}(2 d u s+2 u d s-d s u-s d u-s u d-u s d)$ \\
$p$ & $\tilde{b}_{4}-i \tilde{b}_{5}$ & $2 u u d-d u u-u d u$ \\
$\Xi^{-}$ & $\tilde{b}_{4}+i \tilde{b}_{5}$ & $d s s+s d s-2 s s d$ \\
$n$ & $\tilde{b}_{6}-i \tilde{b}_{7}$ & $d u d+u d d-2 d d u$ \\
$\Xi^{0}$ & $\tilde{b}_{6}+i \tilde{b}_{7}$ & $2 s s u-s u s-u s s$ \\
$\Lambda$ & $\tilde{b}_{8}$ & $\frac{\sqrt{3}}{2}(d s u+s d u-s u d-u s d)$ \\
\hline
\end{tabular}

Table D.2.: Relation of the octet baryons to the components of $\tilde{B}=\tilde{b}_{a} \lambda_{a}$. 


\section{Appendix E.}

\section{Matrix elements of spin-flavour operators}

Matrix elements of spin-flavour operators can be calculated in various ways. In this chapter a method for the calculation of the matrix-elements of one-body spin-flavour operators is presented, which makes the role of quarks as carrier of the baryon quantum numbers more explicit. Despite the lengthy derivation of the results for one-body operators, these result can be easily applied to the matrix elements of arbitrary products of one-body operators.

\section{E.1. Fermionic and bosonic algebras for quarks}

The quark creation- and annihilation operators obey fermionic commutation relations:

$$
\left\{q^{i}, q_{j}^{\dagger}\right\}=\delta_{j}^{i}, \quad\left\{q^{i}, q^{j}\right\}=\left\{q_{i}^{\dagger}, q_{j}^{\dagger}\right\}=0
$$

Here and in the following few lines latin letters represent collective indices of all quantum numbers (spin, flavour and colour). It holds:

$$
\begin{aligned}
q^{l} q_{i}^{\dagger} q_{j}^{\dagger} q_{k}^{\dagger}|0\rangle & =\left(\delta_{i}^{l} q_{j}^{\dagger} q_{k}^{\dagger}-\delta_{j}^{l} q_{i}^{\dagger} q_{k}^{\dagger}+\delta_{k}^{l} q_{i}^{\dagger} q_{j}^{\dagger}\right)|0\rangle, \\
q^{m} q^{l} q_{i}^{\dagger} q_{j}^{\dagger} q_{k}^{\dagger}|0\rangle & =\left(\delta_{i}^{l}\left(\delta_{j}^{m} q_{k}^{\dagger}-\delta_{k}^{m} q_{j}^{\dagger}\right)-\delta_{j}^{l}\left(\delta_{i}^{m} q_{k}^{\dagger}-\delta_{k}^{m} q_{i}^{\dagger}\right)+\delta_{k}^{l}\left(\delta_{i}^{m} q_{j}^{\dagger}-\delta_{j}^{m} q_{i}^{\dagger}\right)\right)|0\rangle, \\
q^{n} q^{m} q^{l} q_{i}^{\dagger} q_{j}^{\dagger} q_{k}^{\dagger}|0\rangle & =\left(\delta_{i}^{l} \delta_{j}^{m} \delta_{k}^{n}-\delta_{i}^{l} \delta_{k}^{m} \delta_{j}^{n}-\delta_{j}^{l} \delta_{i}^{m} \delta_{k}^{n}+\delta_{j}^{l} \delta_{k}^{m} \delta_{i}^{n}+\delta_{k}^{l} \delta_{i}^{m} \delta_{j}^{n}-\delta_{k}^{l} \delta_{j}^{m} \delta_{i}^{n}\right)|0\rangle \\
& =\mathcal{P}_{i j k} \operatorname{sign}(i j k) \delta_{i}^{l} \delta_{j}^{m} \delta_{k}^{n}|0\rangle .
\end{aligned}
$$

$\mathcal{P}_{i j k}$ permutes the indices $i, j$ and $k$ and $\operatorname{sign}(i j k)$ is +1 for cyclic and -1 for anti-cyclic permutations of indices. Further, we introduce useful notation

$$
|i j k\rangle \equiv q_{i}^{\dagger} q_{j}^{\dagger} q_{k}^{\dagger}|0\rangle
$$

which will be used frequently in doing calculations of matrix elements like in

$$
\begin{aligned}
\langle n o p \mid i j k\rangle & =\left\langle 0\left|q^{n} q^{m} q^{l} q_{i}^{\dagger} q_{j}^{\dagger} q_{k}^{\dagger}\right| 0\right\rangle=\mathcal{P}_{i j k} \operatorname{sign}(i j k) \delta_{i}^{l} \delta_{j}^{m} \delta_{k}^{n}, \\
\left\langle n o p\left|q_{o}^{\dagger} q^{p}\right| i j k\right) & \left.\left.\left.=\delta_{i}^{p}\langle\text { nop }| \text { ojk }\right\rangle-\delta_{j}^{p}\langle\text { nop }| \text { oik }\right\rangle+\delta_{k}^{p}\langle\text { nop }| \text { oij }\right\rangle .
\end{aligned}
$$


Calculation of the matrix elements of colour-neutral operators involving colour-neutral states can be greatly simplified by introducing bosonic operators which keep track of spin and flavour indices only. To show this, we separate the indices for the spin and flavour quantum numbers from those describing the colour of quarks. A baryon state is then written as

$$
|B\rangle=\varepsilon^{A B C} \mathcal{B}^{i j k}|i A j B k C\rangle=\varepsilon^{A B C} \mathcal{B}^{i j k} q_{i A}^{\dagger} q_{j B}^{\dagger} q_{k C}^{\dagger}|0\rangle .
$$

Here, the small and big latin letters index the spin-flavour and colour quantum numbers, respectively. The colour part of the wave function is given by the totaly antisymmetric epsilon tensor, the spin-flavour part by the tensor $\mathcal{B}^{i j k}$. Using this notation, the normalisation of baryon states is given by

$$
\begin{aligned}
\left\langle\mathcal{B}^{\prime} \mid \mathcal{B}\right\rangle= & \mathcal{B}_{\text {nop }}^{\prime} \mathcal{B}^{i j k} \varepsilon_{D E F} \varepsilon^{A B C}\left\langle 0\left|q^{n F} q^{m E} q^{l D} q_{i A}^{\dagger} q_{j B}^{\dagger} q_{k C}^{\dagger}\right| 0\right\rangle \\
= & \mathcal{B}_{n o p}^{\prime} \mathcal{B}^{i j k} \varepsilon_{D E F} \varepsilon^{A B C}\left(\begin{array}{ll}
l & \delta_{i}^{l} \delta_{j}^{m} \delta_{k}^{n} \delta_{A}^{D} \delta_{B}^{E} \delta_{C}^{F}-\delta_{i}^{l} \delta_{k}^{m} \delta_{j}^{n} \delta_{A}^{D} \delta_{C}^{E} \delta_{B}^{F}-\delta_{j}^{l} \delta_{i}^{m} \delta_{k}^{n} \delta_{B}^{D} \delta_{A}^{E} \delta_{C}^{F} \\
& \left.\quad+\delta_{j}^{l} \delta_{k}^{m} \delta_{i}^{n} \delta_{B}^{D} \delta_{C}^{E} \delta_{A}^{F}+\delta_{k}^{l} \delta_{i}^{m} \delta_{j}^{n} \delta_{C}^{D} \delta_{A}^{E} \delta_{B}^{F}-\delta_{k}^{l} \delta_{j}^{m} \delta_{i}^{n} \delta_{C}^{D} \delta_{B}^{E} \delta_{A}^{F}\right)
\end{array}\right. \\
= & \mathcal{B}_{\text {nop }}^{\prime} \mathcal{B}^{i j k} \underbrace{\varepsilon_{A B C} \varepsilon^{A B C}}_{=3 !}\left(\delta_{i}^{l} \delta_{j}^{m} \delta_{k}^{n}+\delta_{i}^{l} \delta_{k}^{m} \delta_{j}^{n}+\delta_{j}^{l} \delta_{i}^{m} \delta_{k}^{n}+\delta_{j}^{l} \delta_{k}^{m} \delta_{i}^{n}+\delta_{k}^{l} \delta_{i}^{m} \delta_{j}^{n}+\delta_{k}^{l} \delta_{j}^{m} \delta_{i}^{n}\right) \\
= & \mathcal{B}_{\text {nop }}^{\prime} \mathcal{B}^{i j k} 3 !\langle\text { nop } \mid i j k\rangle,
\end{aligned}
$$

with

$$
\langle n o p \mid i j k\rangle \equiv \mathcal{P}_{i j k} \delta_{i}^{l} \delta_{j}^{m} \delta_{k}^{n}
$$

It holds:

$$
\varepsilon_{D E F} \varepsilon^{A B C}\langle l D m E n F \mid i A j B k C\rangle=3 !\langle n o p \mid i j k\rangle .
$$

Furthermore:

$$
\begin{aligned}
q_{o H}^{\dagger} q^{p H} \varepsilon^{A B C} q_{i A}^{\dagger} q_{j B}^{\dagger} q_{k C}^{\dagger}|0\rangle & =q_{o H}^{\dagger}\left(\delta_{i}^{p} \varepsilon^{H B C} q_{j B}^{\dagger} q_{k C}^{\dagger}-\delta_{j}^{p} \varepsilon^{A H C} q_{i A}^{\dagger} q_{k C}^{\dagger}+\delta_{k}^{p} \varepsilon^{A B H} q_{i A}^{\dagger} q_{j B}^{\dagger}\right)|0\rangle \\
& =\left(\delta_{i}^{p} \varepsilon^{A B C} q_{o A}^{\dagger} q_{j B}^{\dagger} q_{k C}^{\dagger}-\delta_{j}^{p} \varepsilon^{A B C} q_{o B}^{\dagger} q_{i A}^{\dagger} q_{k C}^{\dagger}+\delta_{k}^{p} \varepsilon^{A B C} q_{o C}^{\dagger} q_{i A}^{\dagger} q_{j B}^{\dagger}\right)|0\rangle \\
& =\varepsilon^{A B C}\left(\delta_{i}^{p} q_{o A}^{\dagger} q_{j B}^{\dagger} q_{k C}^{\dagger}+\delta_{j}^{p} q_{o A}^{\dagger} q_{i B}^{\dagger} q_{k C}^{\dagger}+\delta_{k}^{p} q_{o A}^{\dagger} q_{i B}^{\dagger} q_{j C}^{\dagger}\right)|0\rangle \\
& =\varepsilon^{A B C}\left(\delta_{i}^{p}|o A k B k C\rangle+\delta_{j}^{p}|o A i B k C\rangle+\delta_{k}^{p}|o A i B j C\rangle\right) .
\end{aligned}
$$

With the help of the last relation one obtains

$$
\begin{aligned}
\varepsilon_{D E F} & \varepsilon^{A B C}\left\langle l D m E n F\left|q_{o H}^{\dagger} q^{p H}\right| i A j B k C\right\rangle=\varepsilon_{D E F} \varepsilon^{A B C} \times \\
& {\left[\delta_{i}^{p}\langle l D m E n F \mid o A j B k C\rangle+\delta_{j}^{p}\langle l D m E n F \mid o A i B k C\rangle+\delta_{k}^{p}\langle l D m E n F \mid o A i B j C\rangle\right] } \\
& =3 !\left[\delta_{i}^{p}\langle n o p \mid o j k\rangle+\delta_{j}^{p}\langle n o p \mid o i k\rangle+\delta_{k}^{p}\langle\text { nop } \mid o i j\rangle\right] .
\end{aligned}
$$

And finally:

$$
\begin{aligned}
\left\langle\mathcal{B}^{\prime}\left|q_{o H}^{\dagger} q^{p H}\right| \mathcal{B}\right\rangle & =\mathcal{B}_{\text {nop }}^{\prime} \mathcal{B}^{i j k} \varepsilon_{D E F} \varepsilon^{A B C}\left\langle l D m E n F\left|q_{o H}^{\dagger} q^{p H}\right| i A j B k C\right\rangle \\
& \left.\left.\left.=3 ! \mathcal{B}_{\text {nop }}^{\prime} \mathcal{B}^{i j k}\left[\delta_{i}^{p}\langle\text { nop }| \text { ojk }\right\rangle+\delta_{j}^{p}\langle\text { nop }| \text { oik }\right\rangle+\delta_{k}^{p}\langle\text { nop }| \text { oij }\right\rangle\right]
\end{aligned}
$$


Up to the factor 3!, the results in (E.6) and (E.11) can be obtained by using spin-flavour quark operators, which obey the bosonic algebra

$$
\left[q^{i}, q_{j}^{\dagger}\right]=\delta_{j}^{i}, \quad\left[q^{i}, q^{j}\right]=\left[q_{i}^{\dagger}, q_{j}^{\dagger}\right]=0,
$$

and by using baryon states, which are defined in spin-flavour space only:

$$
\left.\left.\mid B)=\mathcal{B}^{i j k} \mid i j k\right)=\mathcal{B}^{i j k} q_{i}^{\dagger} q_{j}^{\dagger} q_{k}^{\dagger} \mid 0\right) .
$$

Round bra- and ket-notation is used here to distinguish the spin-flavour states from states in (E.5) where also the colour quantum numbers are specified. The identities in (E.2) are easily adapted to the bosonic spin-flavour operators:

$$
\begin{aligned}
\left.q^{l} q_{i}^{\dagger} q_{j}^{\dagger} q_{k}^{\dagger} \mid 0\right) & \left.=\left(\delta_{i}^{l} q_{j}^{\dagger} q_{k}^{\dagger}+\delta_{j}^{l} q_{i}^{\dagger} q_{k}^{\dagger}+\delta_{k}^{l} q_{i}^{\dagger} q_{j}^{\dagger}\right) \mid 0\right), \\
\left.q^{m} q^{l} q_{i}^{\dagger} q_{j}^{\dagger} q_{k}^{\dagger} \mid 0\right) & \left.=\left(\delta_{i}^{l}\left(\delta_{j}^{m} q_{k}^{\dagger}+\delta_{k}^{m} q_{j}^{\dagger}\right)+\delta_{j}^{l}\left(\delta_{i}^{m} q_{k}^{\dagger}+\delta_{k}^{m} q_{i}^{\dagger}\right)+\delta_{k}^{l}\left(\delta_{i}^{m} q_{j}^{\dagger}+\delta_{j}^{m} q_{i}^{\dagger}\right)\right) \mid 0\right), \\
\left.q^{n} q^{m} q^{l} q_{i}^{\dagger} q_{j}^{\dagger} q_{k}^{\dagger} \mid 0\right) & \left.=\left(\delta_{i}^{l} \delta_{j}^{m} \delta_{k}^{n}+\delta_{i}^{l} \delta_{k}^{m} \delta_{j}^{n}+\delta_{j}^{l} \delta_{i}^{m} \delta_{k}^{n}+\delta_{j}^{l} \delta_{k}^{m} \delta_{i}^{n}+\delta_{k}^{l} \delta_{i}^{m} \delta_{j}^{n}+\delta_{k}^{l} \delta_{j}^{m} \delta_{i}^{n}\right) \mid 0\right) \\
& \left.=\mathcal{P}_{i j k} \delta_{i}^{l} \delta_{j}^{m} \delta_{k}^{n} \mid 0\right) .
\end{aligned}
$$

\section{E.2. Normalisation of baryon states}

Using bosonic operators, we calculate the normalisation of the spin-flavour states discussed in Appendix D and the action of the operators $\mathbb{1}, J, T$ and $G$, defined in (2.24), on these states. The spin-flavour states for the flavour $S U(3)$-octet $\operatorname{are}^{12}$

$$
\left.\mid a, \chi) \equiv \mathcal{N}_{8} \mathcal{B}_{\alpha a \beta b \gamma c}^{(a, \chi)} q_{\alpha a}^{\dagger} q_{\beta b}^{\dagger} q_{\gamma c}^{\dagger} \mid 0\right)
$$

with

$$
-\mathcal{B}_{\alpha a \beta b \gamma c}^{(a, \chi)} \equiv \varepsilon_{a b g} \lambda_{g c}^{(a)} i \sigma_{\alpha \beta}^{(2)} \delta_{\gamma \chi},
$$

and for the flavour $S U(3)$-decuplet

$$
\left.\left.\mid i j k, \chi) \equiv \mathcal{N}_{10} \mathcal{D}_{\alpha \beta \gamma}^{(\chi)}\left(\delta_{a i} \delta_{b j} \delta_{c k}\right)_{\operatorname{sym}(i j k)} q_{\alpha a}^{\dagger} q_{\beta b}^{\dagger} q_{\gamma c}^{\dagger} \mid 0\right)=\mathcal{N}_{10} \mathcal{D}_{\alpha \beta \gamma}^{(\chi)} q_{\alpha i}^{\dagger} q_{\beta j}^{\dagger} q_{\gamma k}^{\dagger} \mid 0\right),
$$

where the total symmetric tensor $D_{\alpha \beta \gamma}^{(\chi)}$ is given by

$$
D_{\alpha \beta \gamma}^{(\chi)} \equiv\left(\vec{\sigma} i \sigma^{(2)}\right)_{\alpha \beta} \vec{S}_{\gamma \chi}^{\dagger}+\left(\vec{\sigma} i \sigma^{(2)}\right)_{\alpha \gamma} \vec{S}_{\beta \chi}^{\dagger}+\left(\vec{\sigma} i \sigma^{(2)}\right)_{\beta \gamma} \vec{S}_{\alpha \chi}^{\dagger} .
$$

${ }^{1}$ For the sake of simplicity, no use of the co- and contravariant notation for the spin and flavour indices is done in the remainder of this appendix. The summation is done over all indices appearing twice in a single term. There is no danger in doing so, because we are dealing with quarks only (no antiquarks).

${ }^{2}$ For the calcualtions in this chapter, only the first octet component $B_{j}^{i}$ in (D.12), can be considered. The explicit addition of the second octet component $\tilde{B}_{j}^{i}$ and the averaging of them in (E.15) doesn't affect any results. 
The normalisation factors are

$$
\mathcal{N}_{8}=1 / \sqrt{24}, \quad \mathcal{N}_{10}=1 / \sqrt{108}
$$

The corresponding bra-states are

$$
\begin{aligned}
(a, \chi \mid & \equiv \mathcal{N}_{8} \overline{\mathcal{B}}_{\alpha a \beta b \gamma c}^{(a, \chi)}\left(0 \mid q_{\gamma c} q_{\beta b} q_{\alpha a}=\mathcal{N}_{8} \lambda_{g a}^{(a)} \varepsilon_{b c g} i \sigma_{\alpha \beta}^{(2)} \delta_{\gamma \chi}\left(0 \mid q_{\gamma c} q_{\beta b} q_{\alpha a},\right.\right. \\
(i j k, \chi \mid & \equiv \mathcal{N}_{10} \overline{\mathcal{D}}_{\alpha \beta \gamma}^{(\chi)}\left(0 \mid q_{\gamma k} q_{\beta j} q_{\alpha i},\right.
\end{aligned}
$$

with

$$
\overline{\mathcal{D}}_{\alpha \beta \gamma}^{(\chi)} \equiv-\left(i \sigma^{(2)} \vec{\sigma}\right)_{\alpha \beta} \vec{S}_{\chi \gamma}-\left(i \sigma^{(2)} \vec{\sigma}\right)_{\alpha \gamma} \vec{S}_{\chi \beta}-\left(i \sigma^{(2)} \vec{\sigma}\right)_{\beta \gamma} \vec{S}_{\chi \alpha}
$$

The normalisation of the states is

$$
\begin{aligned}
(b, \bar{\chi} \mid a, \chi) & =\mathcal{N}_{8}^{2} \overline{\mathcal{B}}_{\zeta d \eta e \theta f}^{(b, \bar{\chi})} \mathcal{B}_{\alpha a \beta b \gamma c}^{(a, \chi)}\left(0\left|q_{\theta f} q_{\eta e} q_{\zeta d} q_{\alpha a}^{\dagger} q_{\beta b}^{\dagger} q_{\gamma c}^{\dagger}\right| 0\right) \\
& =\mathcal{N}_{8}^{2} \mathcal{B}_{\alpha a \beta b \gamma c}^{(a, \chi)}\left[\overline{\mathcal{B}}_{\alpha a \beta b \gamma c}^{(b, \bar{\chi})}+\overline{\mathcal{B}}_{\alpha a \gamma c \beta b}^{(b, \bar{\chi})}+\overline{\mathcal{B}}_{\beta b \alpha a \gamma c}^{(b, \bar{\chi})}+\overline{\mathcal{B}}_{\beta b \gamma c \alpha a}^{(b, \bar{\chi})}+\overline{\mathcal{B}}_{\gamma c \alpha a \beta b}^{(b, \bar{\chi})}+\overline{\mathcal{B}}_{\gamma c \beta b \alpha a}^{(b, \bar{\chi})}\right] \\
& =\mathcal{N}_{8}^{2} 24 \delta^{a b} \delta_{\bar{\chi} \chi}=\delta^{a b} \delta_{\bar{\chi} \chi},
\end{aligned}
$$

$$
\begin{aligned}
(n o p, \bar{\chi} \mid i j k, \chi) & =\mathcal{N}_{10}^{2} \mathcal{D}_{\alpha \beta \gamma}^{(\chi)} \overline{\mathcal{D}}_{\zeta \eta \theta}^{(\bar{\chi})}\left(0\left|q_{\theta n} q_{\eta m} q_{\zeta l} q_{\alpha i}^{\dagger} q_{\beta j}^{\dagger} q_{\gamma k}^{\dagger}\right| 0\right) \\
& =\mathcal{N}_{10}^{2} \mathcal{D}_{\alpha \beta \gamma}^{(\chi)} \overline{\mathcal{D}}_{\alpha \beta \gamma}^{(\bar{\chi})} 6\left(\delta_{l i} \delta_{m j} \delta_{n k}\right)_{\operatorname{sym}(i j k)} \\
& =\mathcal{N}_{10}^{2} 18 \delta_{\bar{\chi} \chi} 6\left(\delta_{l i} \delta_{m j} \delta_{n k}\right)_{\operatorname{sym}(i j k)}=\delta_{\bar{\chi} \chi}\left(\delta_{l i} \delta_{m j} \delta_{n k}\right)_{\operatorname{sym}(i j k)} .
\end{aligned}
$$

The expected orthogonality of the octet and decuplet states, $(n o p, \bar{\chi} \mid a, \chi)=0$, is verified by an explicit calculation.

\section{E.3. One-body operators}

To evaluate the action of the operators $\mathbb{1}, J, T$ and $G$ on the baryon states, we note first:

$$
\begin{aligned}
\left.q_{\mu r}^{\dagger} q_{\nu s} \mid a, \chi\right) & \left.=\mathcal{N}_{8} \mathcal{B}_{\alpha a \beta b \gamma c}^{(a, \chi)} q_{\mu r}^{\dagger} q_{\nu s} q_{\alpha a}^{\dagger} q_{\beta b}^{\dagger} q_{\gamma c}^{\dagger} \mid 0\right) \\
& \left.=\mathcal{N}_{8} \mathcal{B}_{\alpha a \beta b \gamma c}^{(a, \chi)}\left(\delta_{\nu \alpha} \delta_{s a} q_{\mu r}^{\dagger} q_{\beta b}^{\dagger} q_{\gamma c}^{\dagger}+\delta_{\nu \beta} \delta_{s b} q_{\mu r}^{\dagger} q_{\alpha a}^{\dagger} q_{\gamma c}^{\dagger}+\delta_{\nu \gamma} \delta_{s c} q_{\mu r}^{\dagger} q_{\alpha a}^{\dagger} q_{\beta b}^{\dagger}\right) \mid 0\right) \\
& \left.=\mathcal{N}_{8}\left(\mathcal{B}_{\nu s \beta b \gamma c}^{(a, \chi)} q_{\mu r}^{\dagger} q_{\beta b}^{\dagger} q_{\gamma c}^{\dagger}+\mathcal{B}_{\alpha a \nu s \gamma c}^{(a, \chi)} q_{\mu r}^{\dagger} q_{\alpha a}^{\dagger} q_{\gamma c}^{\dagger}+\mathcal{B}_{\alpha a \beta b \nu s}^{(a, \chi)} q_{\mu r}^{\dagger} q_{\alpha a}^{\dagger} q_{\beta b}^{\dagger}\right) \mid 0\right) \\
& \left.=\mathcal{N}_{8}\left(2 \mathcal{B}_{\nu s \beta b \gamma c}^{(a, \chi)} q_{\mu r}^{\dagger} q_{\beta b}^{\dagger} q_{\gamma c}^{\dagger}+\mathcal{B}_{\alpha a \beta b \nu s}^{(a, \chi)} q_{\mu r}^{\dagger} q_{\alpha a}^{\dagger} q_{\beta b}^{\dagger}\right) \mid 0\right), \\
\left.q_{\mu r}^{\dagger} q_{\nu s} \mid i j k, \chi\right) & \left.=\mathcal{N}_{10} \mathcal{D}_{\alpha \beta \gamma}^{(\chi)} q_{\mu r}^{\dagger} q_{\nu s} q_{\alpha i}^{\dagger} q_{\beta j}^{\dagger} q_{\gamma k}^{\dagger} \mid 0\right) \\
& \left.=\mathcal{N}_{10} \mathcal{D}_{\alpha \beta \gamma}^{(\chi)}\left(\delta_{\nu \alpha} \delta_{s i} q_{\mu r}^{\dagger} q_{\beta j}^{\dagger} q_{\gamma k}^{\dagger}+\delta_{\nu \beta} \delta_{s j} q_{\mu r}^{\dagger} q_{\alpha i}^{\dagger} q_{\gamma k}^{\dagger}+\delta_{\nu \gamma} \delta_{s k} q_{\mu r}^{\dagger} q_{\alpha i}^{\dagger} q_{\beta j}^{\dagger}\right) \mid 0\right) \\
& \left.=\mathcal{N}_{10}\left(\mathcal{D}_{\nu \beta \gamma}^{(\chi)} \delta_{s i} q_{\mu r}^{\dagger} q_{\beta j}^{\dagger} q_{\gamma k}^{\dagger}+\mathcal{D}_{\alpha \nu \gamma}^{(\chi)} \delta_{s j} q_{\mu r}^{\dagger} q_{\alpha i}^{\dagger} q_{\gamma k}^{\dagger}+\mathcal{D}_{\alpha \beta \nu}^{(\chi)} \delta_{s k} q_{\mu r}^{\dagger} q_{\alpha i}^{\dagger} q_{\beta j}^{\dagger}\right) \mid 0\right) .
\end{aligned}
$$


The action of the operators on the states is obtained by contracting the above results with the appropriate spin-flavour tensors. The contractions for the octet states are:

$$
\begin{aligned}
& \left.\mathbb{1} \mid a, \chi)=\delta_{\mu \nu} \delta_{r s} q_{\mu r}^{\dagger} q_{\nu s} \mid a, \chi\right) \\
& \left.=\mathcal{N}_{8}\left(2 \mathcal{B}_{\mu r \beta b \gamma c}^{(a, \chi)} q_{\mu r}^{\dagger} q_{\beta b}^{\dagger} q_{\gamma c}^{\dagger}+\mathcal{B}_{\alpha a \beta b \mu r}^{(a, \chi)} q_{\mu r}^{\dagger} q_{\alpha a}^{\dagger} q_{\beta b}^{\dagger}\right) \mid 0\right) \\
& =3 \mid a, \chi) \text {, } \\
& \left.\left.J^{k} \mid a, \chi\right)=\frac{1}{2} \sigma_{\mu \nu}^{(k)} \delta_{r s} q_{\mu r}^{\dagger} q_{\nu s} \mid a, \chi\right) \\
& \left.=\mathcal{N}_{8} \frac{1}{2} \sigma_{\mu \nu}^{(k)}\left(2 \mathcal{B}_{\nu r \beta b \gamma c}^{(a, \chi)} q_{\mu r}^{\dagger} q_{\beta b}^{\dagger} q_{\gamma c}^{\dagger}+\mathcal{B}_{\alpha a \beta b \nu r}^{(a, \chi)} q_{\mu r}^{\dagger} q_{\alpha a}^{\dagger} q_{\beta b}^{\dagger}\right) \mid 0\right) \\
& \left.=-\mathcal{N}_{8} \frac{1}{2}\left(2 \varepsilon_{r b g} \lambda_{c g}^{(a)}\left(\sigma^{(k)} i \sigma^{(2)}\right)_{\mu \beta} \delta_{\gamma \chi} q_{\mu r}^{\dagger} q_{\beta b}^{\dagger} q_{\gamma c}^{\dagger}+\varepsilon_{a b g} \lambda_{r g}^{(a)} i \sigma_{\alpha \beta}^{(2)} \sigma_{\mu \chi}^{(k)} q_{\alpha a}^{\dagger} q_{\beta b}^{\dagger} q_{\mu r}^{\dagger}\right) \mid 0\right) \\
& \left.=-\mathcal{N}_{8} \frac{1}{2}\left(0+\sigma_{\bar{\chi} \chi}^{(k)} \varepsilon_{a b g} \lambda_{r g}^{(a)} i \sigma_{\alpha \beta}^{(2)} \delta_{\mu \bar{\chi}} q_{\alpha a}^{\dagger} q_{\beta b}^{\dagger} q_{\mu r}^{\dagger}\right) \mid 0\right) \\
& \left.=\frac{1}{2} \sigma_{\bar{\chi} \chi}^{(k)} \mid a, \bar{\chi}\right) \text {, } \\
& \left.\left.T^{b} \mid a, \chi\right)=\delta_{\mu \nu} \frac{1}{2} \lambda_{r s}^{(b)} q_{\mu r}^{\dagger} q_{\nu s} \mid a, \chi\right) \\
& \left.=\mathcal{N}_{8} \frac{1}{2} \lambda_{r s}^{(b)}\left(2 \mathcal{B}_{\mu s \beta b \gamma c}^{(a, \chi)} q_{\mu r}^{\dagger} q_{\beta b}^{\dagger} q_{\gamma c}^{\dagger}+\mathcal{B}_{\gamma c \beta b \mu s}^{(a, \chi)} q_{\mu r}^{\dagger} q_{\beta b}^{\dagger} q_{\gamma c}^{\dagger}\right) \mid 0\right) \\
& \left.=-\mathcal{N}_{8} \frac{1}{2} \lambda_{r s}^{(b)}\left(2 \varepsilon_{s b g} \lambda_{c g}^{(a)} i \sigma_{\mu \beta}^{(2)} \delta_{\gamma \chi}+\varepsilon_{c b g} \lambda_{s g}^{(a)} i \sigma_{\gamma \beta}^{(2)} \delta_{\mu \chi}\right) q_{\mu r}^{\dagger} q_{\beta b}^{\dagger} q_{\gamma c}^{\dagger} \mid 0\right) \\
& \left.=-\mathcal{N}_{8} \frac{1}{2}\left(2 \varepsilon_{s b g} \lambda_{r s}^{(b)} \lambda_{c g}^{(a)}+\left(\lambda^{(b)} \lambda^{(a)}\right)_{c g} \varepsilon_{r b g}\right) i \sigma_{\mu \beta}^{(2)} \delta_{\gamma \chi} q_{\mu r}^{\dagger} q_{\beta b}^{\dagger} q_{\gamma c}^{\dagger} \mid 0\right) \\
& \left.\stackrel{\star}{=}-\mathcal{N}_{8} \frac{1}{2}\left(-\frac{2}{3} \delta^{b a} \delta_{c g}-\left(d^{b a c}-i f^{b a c}\right) \lambda_{c g}^{(c)}\right) \varepsilon_{r b g} i \sigma_{\mu \beta}^{(2)} \delta_{\gamma \chi} q_{\mu r}^{\dagger} q_{\beta b}^{\dagger} q_{\gamma c}^{\dagger} \mid 0\right) \\
& \left.-\mathcal{N}_{8} \frac{1}{2}\left(\frac{2}{3} \delta^{b a} \delta_{c g}+\left(d^{b a c}+i f^{b a c}\right) \lambda_{c g}^{(c)}\right) \varepsilon_{r b g} i \sigma_{\mu \beta}^{(2)} \delta_{\gamma \chi} q_{\mu r}^{\dagger} q_{\beta b}^{\dagger} q_{\gamma c}^{\dagger} \mid 0\right) \\
& \left.=-\mathcal{N}_{8} i f^{b a c} \varepsilon_{r b g} \lambda_{c g}^{(c)} i \sigma_{\mu \beta}^{(2)} \delta_{\gamma \chi} q_{\mu r}^{\dagger} q_{\beta b}^{\dagger} q_{\gamma c}^{\dagger} \mid 0\right) \\
& \left.=i f^{b a c} \mid c, \chi\right) \text {, } \\
& \left.\left.G^{k b} \mid a, \chi\right)=\frac{1}{2} \sigma_{\mu \nu}^{(k)} \frac{1}{2} \lambda_{r s}^{(b)} q_{\mu r}^{\dagger} q_{\nu s} \mid a, \chi\right) \\
& \left.=\mathcal{N}_{8} \frac{1}{2} \lambda_{r s}^{(b)} \frac{1}{2} \sigma_{\mu \nu}^{(k)}\left(2 \mathcal{B}_{\nu s \beta b \gamma c}^{(a, \chi)} q_{\mu r}^{\dagger} q_{\beta b}^{\dagger} q_{\gamma c}^{\dagger}+\mathcal{B}_{\gamma c \beta b \nu s}^{(a, \chi)} q_{\mu r}^{\dagger} q_{\beta b}^{\dagger} q_{\gamma c}^{\dagger}\right) \mid 0\right) \\
& \left.=-\mathcal{N}_{8} \frac{1}{2} \lambda_{r s}^{(b)} \frac{1}{2} \sigma_{\mu \nu}^{(k)}\left(2 \varepsilon_{s b g} \lambda_{c g}^{(a)} i \sigma_{\nu \beta}^{(2)} \delta_{\gamma \chi}+\varepsilon_{c b g} \lambda_{s g}^{(a)} i \sigma_{\gamma \beta}^{(2)} \delta_{\nu \chi}\right) q_{\mu r}^{\dagger} q_{\beta b}^{\dagger} q_{\gamma c}^{\dagger} \mid 0\right) \\
& \left.=-\mathcal{N}_{8} \frac{1}{4}\left(2 \varepsilon_{s b g} \lambda_{r s}^{(b)} \lambda_{c g}^{(a)}\left(\sigma^{(k)} i \sigma^{(2)}\right)_{\mu \beta} \delta_{\gamma \chi}+\varepsilon_{c b g}\left(\lambda^{(b)} \lambda^{(a)}\right)_{r g} \sigma_{\mu \chi}^{(k)} i \sigma_{\gamma \beta}^{(2)}\right) q_{\mu r}^{\dagger} q_{\beta b}^{\dagger} q_{\gamma c}^{\dagger} \mid 0\right) \\
& \stackrel{\star}{=}\left(-\left(\frac{1}{2} d^{b a c}+\frac{1}{3} i f^{b a c}\right) \sigma_{\bar{\chi} \chi}^{(i)} \mathcal{N}_{8} \varepsilon_{r b g} \lambda_{c g}^{(c)} i \sigma_{\mu \beta}^{(2)} \delta_{\gamma \bar{\chi}}\right. \\
& \left.\left.+\frac{1}{2 \sqrt{2}}\left(\varepsilon_{r o p} \lambda_{b o}^{(b)} \lambda_{c p}^{(a)}\right)_{\operatorname{sym}(r b c)} S_{\bar{\chi} \chi}^{(i)} \mathcal{N}_{10} \mathcal{D}_{\mu \beta \gamma}^{(\bar{\chi})}\right) q_{\mu r}^{\dagger} q_{\beta b}^{\dagger} q_{\gamma c}^{\dagger} \mid 0\right), \\
& \left.\left.=\left(\frac{1}{2} d^{b a c}+\frac{1}{3} i f^{b a c}\right) \sigma_{\bar{\chi} \chi}^{(k)} \mid c, \bar{\chi}\right)+\frac{1}{2 \sqrt{2}}\left(\varepsilon_{i o p} \lambda_{j o}^{(b)} \lambda_{k p}^{(a)}\right)_{\operatorname{sym}(i j k)} S_{\bar{\chi} \chi}^{(k)} \mid i j k, \bar{\chi}\right) .
\end{aligned}
$$


In the step $\star$ in (E.26) the use of the following relations for the $\lambda$-matrices was made:

$$
\begin{aligned}
\lambda^{(b)} \lambda^{(a)} & =\frac{2}{3} \delta^{b a}+\left(d^{b a c}+i f^{b a c}\right) \lambda^{(c)} \\
\varepsilon_{i l m} \lambda_{j l}^{(b)} \lambda_{k m}^{(a)}-\varepsilon_{j l m} \lambda_{i l}^{(b)} \lambda_{k m}^{(a)} & =-\left(\frac{2}{3} \delta^{b a} \delta_{k m}+\left(d^{b a c}-i f^{b a c}\right) \lambda_{k m}^{(c)}\right) \varepsilon_{i j m} .
\end{aligned}
$$

By noting the antisymmetry of $\sigma^{(2)}$, direct application of the last line leads to

$$
\begin{aligned}
\varepsilon_{s b g} \lambda_{r s}^{(b)} \lambda_{c g}^{(a)} \sigma_{\mu \beta}^{(2)} q_{\mu r}^{\dagger} q_{\beta b}^{\dagger} q_{\gamma c}^{\dagger} & =\frac{1}{2}\left(\varepsilon_{s b g} \lambda_{r s}^{(b)} \lambda_{c g}^{(a)}-\varepsilon_{s r g} \lambda_{b s}^{(b)} \lambda_{c g}^{(a)}\right) \sigma_{\mu \beta}^{(2)} q_{\mu r}^{\dagger} q_{\beta b}^{\dagger} q_{\gamma c}^{\dagger} \\
& =-\frac{1}{2}\left(\frac{2}{3} \delta^{b a} \delta_{c g}+\left(d^{b a c}-i f^{b a c}\right) \lambda_{c g}^{(c)}\right) \varepsilon_{r b g} \sigma_{\mu \beta}^{(2)} q_{\mu r}^{\dagger} q_{\beta b}^{\dagger} q_{\gamma c}^{\dagger} .
\end{aligned}
$$

Similar, the step $\star$ in (E.27) involves additional algebraical recasting, which brings no further insight here and is ommited therefore.

The action of the operators on the decuplet states is obtained in a similar manner:

$$
\begin{aligned}
\mathbb{1} \mid i j k, \chi) & \left.=\delta_{\mu \nu} \delta_{r s} q_{\mu r}^{\dagger} q_{\nu s} \mid i j k, \chi\right) \\
& \left.=\mathcal{N}_{10}\left(\mathcal{D}_{\mu \beta \gamma}^{(\chi)} q_{\mu i}^{\dagger} q_{\beta j}^{\dagger} q_{\gamma k}^{\dagger}+\mathcal{D}_{\alpha \mu \gamma}^{(\chi)} q_{\mu j}^{\dagger} q_{\alpha i}^{\dagger} q_{\gamma k}^{\dagger}+\mathcal{D}_{\alpha \beta \mu}^{(\chi)} q_{\mu k}^{\dagger} q_{\alpha i}^{\dagger} q_{\beta j}^{\dagger}\right) \mid 0\right) \\
& =3 \mid i j k, \chi),
\end{aligned}
$$

$$
\begin{aligned}
& \left.\left.J^{k} \mid i j k, \chi\right)=\frac{1}{2} \sigma_{\mu \nu}^{(k)} \delta_{r s} q_{\mu r}^{\dagger} q_{\nu s} \mid i j k, \chi\right) \\
& \left.=\frac{1}{2} \mathcal{N}_{10}\left(\sigma_{\mu \nu}^{(k)} \mathcal{D}_{\nu \beta \gamma}^{(\chi)} q_{\mu i}^{\dagger} q_{\beta j}^{\dagger} q_{\gamma k}^{\dagger}+\sigma_{\mu \nu}^{(k)} \mathcal{D}_{\alpha \nu \gamma}^{(\chi)} q_{\mu j}^{\dagger} q_{\alpha i}^{\dagger} q_{\gamma k}^{\dagger}+\sigma_{\mu \nu}^{(k)} \mathcal{D}_{\alpha \beta \nu}^{(\chi)} q_{\mu k}^{\dagger} q_{\alpha i}^{\dagger} q_{\beta j}^{\dagger}\right) \mid 0\right) \\
& \left.=\frac{1}{2} \mathcal{N}_{10}\left(\sigma_{\mu \nu}^{(k)} \mathcal{D}_{\nu \beta \gamma}^{(\chi)} q_{\mu i}^{\dagger} q_{\beta j}^{\dagger} q_{\gamma k}^{\dagger}+\sigma_{\beta \nu}^{(k)} \mathcal{D}_{\nu \mu \gamma}^{(\chi)} q_{\beta j}^{\dagger} q_{\mu i}^{\dagger} q_{\gamma k}^{\dagger}+\sigma_{\gamma \nu}^{(k)} \mathcal{D}_{\nu \beta \mu}^{(\chi)} q_{\gamma k}^{\dagger} q_{\mu i}^{\dagger} q_{\beta j}^{\dagger}\right) \mid 0\right) \\
& =\frac{i}{2} \mathcal{N}_{10}\left[\left(\sigma^{(k)} \sigma^{(i)} \sigma^{(2)}\right)_{\mu \beta} S_{\gamma \chi}^{(i) \dagger}+\left(\sigma^{(k)} \sigma^{(i)} \sigma^{(2)}\right)_{\mu \gamma} S_{\beta \chi}^{(i) \dagger}+\left(\sigma^{(i)} \sigma^{(2)}\right)_{\beta \gamma}\left(\sigma^{(k)} S^{(i) \dagger}\right)_{\mu \chi}\right. \\
& -\left(\sigma^{(i)} \sigma^{(k)} \sigma^{(2)}\right)_{\mu \beta} S_{\gamma \chi}^{(i) \dagger}+\left(\sigma^{(i)} \sigma^{(2)}\right)_{\mu \gamma}\left(\sigma^{(k)} S^{(i) \dagger}\right)_{\beta \chi}+\left(\sigma^{(k)} \sigma^{(i)} \sigma^{(2)}\right)_{\beta \gamma} S_{\mu \chi}^{(i) \dagger} \\
& \left.+\left(\sigma^{(i)} \sigma^{(2)}\right)_{\mu \beta}\left(\sigma^{(k)} S^{(i) \dagger}\right)_{\gamma \chi}-\left(\sigma^{(i)} \sigma^{(k)} \sigma^{(2)}\right)_{\mu \gamma} S_{\beta \chi}^{(i) \dagger}-\left(\sigma^{(i)} \sigma^{(k)} \sigma^{(2)}\right)_{\beta \gamma} S_{\mu \chi}^{(i) \dagger}\right] \\
& \left.\times q_{\mu i}^{\dagger} q_{\beta j}^{\dagger} q_{\gamma k}^{\dagger} \mid 0\right) \\
& =\frac{i}{2} \mathcal{N}_{10}\left[\left(\sigma^{(k)} \sigma^{(i)} \sigma^{(2)}\right)_{\mu \beta} S_{\gamma \chi}^{(i) \dagger}-\left(\sigma^{(i)} \sigma^{(k)} \sigma^{(2)}\right)_{\mu \beta} S_{\gamma \chi}^{(i) \dagger}+\left(\sigma^{(i)} \sigma^{(2)}\right)_{\mu \beta}\left(\sigma^{(k)} S^{(i) \dagger}\right)_{\gamma \chi}\right. \\
& \left(\sigma^{(k)} \sigma^{(i)} \sigma^{(2)}\right)_{\mu \gamma} S_{\beta \chi}^{(i) \dagger}-\left(\sigma^{(i)} \sigma^{(k)} \sigma^{(2)}\right)_{\mu \gamma} S_{\beta \chi}^{(i) \dagger}+\left(\sigma^{(i)} \sigma^{(2)}\right)_{\mu \gamma}\left(\sigma^{(k)} S^{(i) \dagger}\right)_{\beta \chi} \\
& \left.\left(\sigma^{(k)} \sigma^{(i)} \sigma^{(2)}\right)_{\beta \gamma} S_{\mu \chi}^{(i) \dagger}-\left(\sigma^{(i)} \sigma^{(k)} \sigma^{(2)}\right)_{\beta \gamma} S_{\mu \chi}^{(i) \dagger}+\left(\sigma^{(i)} \sigma^{(2)}\right)_{\beta \gamma}\left(\sigma^{(k)} S^{(i) \dagger}\right)_{\mu \chi}\right] \\
& \left.\times q_{\mu i}^{\dagger} q_{\beta j}^{\dagger} q_{\gamma k}^{\dagger} \mid 0\right) \\
& \stackrel{\star}{=} \frac{3}{2} \mathcal{N}_{10}\left(\vec{S} \sigma^{(k)} \vec{S}^{\dagger}\right)_{\bar{\chi} \chi} \\
& \left.\times\left[\left(\sigma^{(i)} i \sigma^{(2)}\right)_{\mu \beta} S_{\gamma \bar{\chi}}^{(i) \dagger}+\left(\sigma^{(i)} i \sigma^{(2)}\right)_{\mu \gamma} S_{\beta \bar{\chi}}^{(i) \dagger}+\left(\sigma^{(i)} i \sigma^{(2)}\right)_{\beta \gamma} S_{\mu \bar{\chi}}^{(i) \dagger}\right] q_{\mu i}^{\dagger} q_{\beta j}^{\dagger} q_{\gamma k}^{\dagger} \mid 0\right) \\
& \left.\left.=\frac{3}{2}\left(\vec{S} \sigma^{(k)} \vec{S}^{\dagger}\right)_{\bar{\chi} \chi} \mathcal{N}_{10} \mathcal{D}_{\mu \beta \gamma}^{(\bar{\chi})} q_{\mu i}^{\dagger} q_{\beta j}^{\dagger} q_{\gamma k}^{\dagger} \mid 0\right)=\frac{3}{2}\left(\vec{S} \sigma^{(k)} \vec{S}^{\dagger}\right)_{\bar{\chi} \chi} \mid i j k, \bar{\chi}\right) .
\end{aligned}
$$


The identity, used in the step $\star$, is proven as follows:

$$
\begin{aligned}
\left(\sigma^{(k)} \sigma^{(i)}\right. & \left.\sigma^{(2)}\right)_{\mu \beta} S_{\gamma \chi}^{(i) \dagger}-\left(\sigma^{(i)} \sigma^{(k)} \sigma^{(2)}\right)_{\mu \beta} S_{\gamma \chi}^{(i) \dagger}+\left(\sigma^{(i)} \sigma^{(2)}\right)_{\mu \beta}\left(\sigma^{(k)} S^{(i) \dagger}\right)_{\gamma \chi} \\
& =\left(\left[\sigma^{(k)}, \sigma^{(i)}\right] \sigma^{(2)}\right)_{\mu \beta} S_{\gamma \chi}^{(i) \dagger}+\left(\sigma^{(i)} \sigma^{(2)}\right)_{\mu \beta}\left(\sigma^{(k)} S^{(i) \dagger}\right)_{\gamma \chi} \\
& =2 i \varepsilon_{k i j}\left(\sigma^{(j)} \sigma^{(2)}\right)_{\mu \beta} S_{\gamma \chi}^{(i) \dagger}+\left(\sigma^{(i)} \sigma^{(2)}\right)_{\mu \beta}\left(\sigma^{(k)} S^{(i) \dagger}\right)_{\gamma \chi} \\
& =\left(2 i \varepsilon_{j i k} S^{(j) \dagger}+\sigma^{(k)} S^{(i) \dagger}\right)_{\gamma \chi}\left(\sigma^{(i)} \sigma^{(2)}\right)_{\mu \beta} \\
& =3\left(\vec{S} \sigma^{(k)} \vec{S}^{\dagger}\right)_{\bar{\chi} \chi} S_{\gamma \bar{\chi}}^{(i) \dagger}\left(\sigma^{(i)} \sigma^{(2)}\right)_{\mu \beta} .
\end{aligned}
$$

The identity $\left(2 i \varepsilon_{j i k} S^{(j) \dagger}+\sigma^{(k)} S^{(i) \dagger}\right)=3 S^{(i) \dagger}\left(\vec{S} \sigma^{(k)} \vec{S}^{\dagger}\right)$ is shown to be valid by multiplying both sides by $S^{(i)}$, summing over $i$ and using the properties of the $S$-and $\sigma$-matrices summarised in Appendix F:

$$
\begin{aligned}
S^{(i)}\left(2 i \varepsilon_{j i k} S^{(j) \dagger}+\sigma^{(k)} S^{(i) \dagger}\right) & =S^{(i)} 3 S^{(i) \dagger}\left(\vec{S} \sigma^{(k)} \vec{S}^{\dagger}\right) \\
2 i \varepsilon_{j i k} S^{(i)} S^{(j) \dagger}+\vec{S} \sigma^{(k)} \vec{S}^{\dagger} & =3\left(\vec{S} \vec{S}^{\dagger}\right)\left(\vec{S} \sigma^{(k)} \vec{S}^{\dagger}\right) \\
2 \vec{S} \sigma^{(k)} \vec{S}^{\dagger}+\vec{S} \sigma^{(k)} \vec{S}^{\dagger} & =3\left(\vec{S} \sigma^{(k)} \vec{S}^{\dagger}\right) .
\end{aligned}
$$

For the $T$ operator one has:

$$
\begin{aligned}
& \left.\left.T^{a} \mid i j k, \chi\right)=\delta_{\mu \nu} \frac{1}{2} \lambda_{r s}^{(a)} q_{\mu r}^{\dagger} q_{\nu s} \mid i j k, \chi\right) \\
& \left.=\frac{1}{2} \mathcal{N}_{10}\left(\lambda_{r i}^{(a)} \mathcal{D}_{\mu \beta \gamma}^{(\chi)} q_{\mu r}^{\dagger} q_{\beta j}^{\dagger} q_{\gamma k}^{\dagger}+\lambda_{r j}^{(a)} \mathcal{D}_{\alpha \mu \gamma}^{(\chi)} q_{\mu r}^{\dagger} q_{\alpha i}^{\dagger} q_{\gamma k}^{\dagger}+\lambda_{r k}^{(a)} \mathcal{D}_{\alpha \beta \mu}^{(\chi)} q_{\mu r}^{\dagger} q_{\alpha i}^{\dagger} q_{\beta j}^{\dagger}\right) \mid 0\right) \\
& \left.\left.\left.=\frac{1}{2}\left(\lambda_{r i}^{(a)} \mid r j k, \chi\right)+\lambda_{r j}^{(a)} \mid i r k, \chi\right)+\lambda_{r k}^{(a)} \mid i j r, \chi\right)\right) \\
& \left.\left.\left.\left.\stackrel{\star}{=} \frac{1}{4} \lambda_{r i}^{(a)}(\mid r j k, \chi)+\mid r k j, \chi\right)\right)+\frac{1}{4} \lambda_{r j}^{(a)}(\mid r i k, \chi)+\mid r k i, \chi\right)\right) \\
& \left.\left.+\frac{1}{4} \lambda_{r k}^{(a)}(\mid r i j, \chi)+\mid r j i, \chi\right)\right) \\
& =\frac{1}{4}\left(\lambda_{l i}^{(a)} \delta_{m j} \delta_{n k}+\lambda_{l i}^{(a)} \delta_{m k} \delta_{n j}+\lambda_{l j}^{(a)} \delta_{m i} \delta_{n k}+\lambda_{l j}^{(a)} \delta_{m k} \delta_{n i}\right. \\
& \left.\left.+\lambda_{l k}^{(a)} \delta_{m i} \delta_{n j}+\lambda_{l k}^{(a)} \delta_{m j} \delta_{n i}\right) \mid n o p, \chi\right) \\
& \left.=\frac{3}{2}\left(\lambda_{l i}^{(a)} \delta_{m j} \delta_{n k}\right)_{\operatorname{sym}(i j k)} \mid n o p, \chi\right) \text {. }
\end{aligned}
$$

In the step $\star$ the total symmetry in the flavour part of the decuplet states was used. Finally, the action of $G$ on the decuplet states is given by

$$
\begin{aligned}
&\left.G^{k a} \mid i j k, \chi\right)=\left.\frac{1}{2} \sigma_{\mu \nu}^{(k)} \frac{1}{2} \lambda_{r s}^{(a)} q_{\mu r}^{\dagger} q_{\nu s} \mid i j k, \chi\right)=\frac{1}{4} \mathcal{N}_{10} \sigma_{\mu \nu}^{(k)}\left(\lambda_{r i}^{(a)} \mathcal{D}_{\nu \beta \gamma}^{(\chi)} q_{\mu r}^{\dagger} q_{\beta j}^{\dagger} q_{\gamma k}^{\dagger}\right. \\
&\left.\left.\quad+\lambda_{r j}^{(a)} \mathcal{D}_{\alpha \nu \gamma}^{(\chi)} q_{\mu r}^{\dagger} q_{\alpha i}^{\dagger} q_{\gamma k}^{\dagger}+\lambda_{r k}^{(a)} \mathcal{D}_{\alpha \beta \nu}^{(\chi)} q_{\mu r}^{\dagger} q_{\alpha i}^{\dagger} q_{\beta j}^{\dagger}\right) \mid 0\right) \\
&\left.=\frac{1}{4} \mathcal{N}_{10} \sigma_{\mu \nu}^{(k)} \mathcal{D}_{\nu \beta \gamma}^{(\chi)}\left(\lambda_{r i}^{(a)} q_{\mu r}^{\dagger} q_{\beta j}^{\dagger} q_{\gamma k}^{\dagger}+\lambda_{r j}^{(a)} q_{\mu r}^{\dagger} q_{\beta i}^{\dagger} q_{\gamma k}^{\dagger}+\lambda_{r k}^{(a)} q_{\mu r}^{\dagger} q_{\beta j}^{\dagger} q_{\gamma i}^{\dagger}\right) \mid 0\right) \\
&\left.=\frac{1}{4} \mathcal{N}_{10} \sigma_{\mu \nu}^{(k)} \mathcal{D}_{\nu \beta \gamma}^{(\chi)}\left(\lambda_{l i}^{(a)} \delta_{m j} \delta_{n k}+\lambda_{l j}^{(a)} \delta_{m i} \delta_{n k}+\lambda_{l k}^{(a)} \delta_{m j} \delta_{n i}\right) q_{\mu l}^{\dagger} q_{\beta m}^{\dagger} q_{\gamma n}^{\dagger} \mid 0\right)
\end{aligned}
$$




$$
\begin{aligned}
= & \left.\frac{3}{4} \mathcal{N}_{10} \sigma_{\mu \nu}^{(k)} \mathcal{D}_{\nu \beta \gamma}^{(\chi)}\left(\lambda_{l i}^{(a)} \delta_{m j} \delta_{n k}\right)_{\mathrm{sym}(i j k)} q_{\mu l}^{\dagger} q_{\beta m}^{\dagger} q_{\gamma n}^{\dagger} \mid 0\right) \\
= & \left(\frac{3}{4}\left(\lambda_{l i}^{(a)} \delta_{m j} \delta_{n k}\right)_{\operatorname{sym}(i j k)}\left(\vec{S} \sigma^{(k)} \vec{S}^{\dagger}\right)_{\bar{\chi} \chi} \mathcal{N}_{10} \mathcal{D}_{\mu \beta \gamma}^{(\bar{\chi})}\right. \\
& \left.\left.-\frac{1}{2 \sqrt{2}}\left(\varepsilon_{i o p} \lambda_{o j}^{(a)} \lambda_{p k}^{(b)}\right)_{\operatorname{sym}(i j k)} S_{\bar{\chi} \chi}^{(k) \dagger} \mathcal{N}_{8} \varepsilon_{l m g} \lambda_{n g}^{(b)} i \sigma_{\mu \beta}^{(2)} \delta_{\gamma \bar{\chi}}\right) q_{\mu l}^{\dagger} q_{\beta m}^{\dagger} q_{\gamma n}^{\dagger} \mid 0\right) \\
= & \left.\frac{3}{4}\left(\lambda_{l i}^{(a)} \delta_{m j} \delta_{n k}\right)_{\operatorname{sym}(i j k)}\left(\vec{S} \sigma^{(k)} \vec{S}^{\dagger}\right)_{\bar{\chi} \chi} \mid n o p, \bar{\chi}\right) \\
& \left.+\frac{1}{2 \sqrt{2}}\left(\varepsilon_{i o p} \lambda_{o j}^{(a)} \lambda_{p k}^{(b)}\right)_{\operatorname{sym}(i j k)} S_{\bar{\chi} \chi}^{(k) \dagger} \mid b, \bar{\chi}\right) .
\end{aligned}
$$

We summarise:

$$
\begin{aligned}
\left.J^{i} \mid a, \chi\right) & \left.=\frac{1}{2} \sigma_{\bar{\chi} \chi}^{(i)} \mid a, \bar{\chi}\right), \\
\left.T^{a} \mid b, \chi\right) & \left.=i f^{b c a} \mid c, \chi\right), \\
\left.G^{i a} \mid b, \chi\right) & \left.\left.=\frac{1}{2} \sigma_{\bar{\chi} \chi}^{(i)} \Lambda_{b}^{a, c} \mid c, \bar{\chi}\right)+\frac{1}{2 \sqrt{2}} S_{\bar{\chi} \chi}^{(i)} \Lambda_{b}^{a, k l m} \mid k l m, \bar{\chi}\right), \\
\left.J^{i} \mid k l m, \chi\right) & \left.=\frac{3}{2}\left(\vec{S} \sigma^{(i)} \vec{S}^{\dagger}\right)_{\bar{\chi} \chi} \mid k l m, \bar{\chi}\right), \\
\left.T^{a} \mid k l m, \chi\right) & \left.=\frac{3}{2} \Lambda_{k l m}^{a, n o p} \mid n o p, \chi\right), \\
\left.G^{i a} \mid k l m, \chi\right) & \left.\left.=\frac{3}{4}\left(\vec{S} \sigma^{(i)} \vec{S}^{\dagger}\right)_{\bar{\chi} \chi} \Lambda_{k l m}^{a, n o p} \mid n o p, \bar{\chi}\right)+\frac{1}{2 \sqrt{2}} S_{\bar{\chi} \chi}^{(i) \dagger} \Lambda_{k l m}^{a, b} \mid b, \bar{\chi}\right),
\end{aligned}
$$

with the flavor transition tensors

$$
\begin{aligned}
\Lambda_{b}^{a, c} & =\left(d^{a b c}+\frac{2}{3} i f^{a b c}\right), & \Lambda_{b}^{a, k l m} & =\left(\varepsilon_{i j k} \lambda_{l i}^{(a)} \lambda_{m j}^{(b)}\right)_{\operatorname{sym}(k l m)}, \\
\Lambda_{k l m}^{a, n o p} & =\left(\lambda_{n k}^{(a)} \delta_{o l} \delta_{p m}\right)_{\operatorname{sym}(k l m)}, & \Lambda_{k l m}^{a, b} & =\left(\varepsilon_{i j k} \lambda_{i l}^{(a)} \lambda_{j m}^{(b)}\right)_{\operatorname{sym}(k l m)} .
\end{aligned}
$$

\section{E.4. Two-body operators, I}

Matrix elements of arbitrary products of one-body operators can be obtained by consecutive application of (E.32). Symmetric combinations of two one-body operators evaluated between the octet states are:

$$
\begin{aligned}
\left(d, \bar{\chi}\left|\left\{J^{i}, J^{j}\right\}\right| c, \chi\right) & =\frac{1}{2} \delta_{i j} \delta_{\bar{\chi} \chi} \delta_{c d}, \\
\left(d, \bar{\chi}\left|\left\{J^{i}, T^{a}\right\}\right| c, \chi\right) & =\sigma_{\bar{\chi} \chi}^{(i)} i f^{a c d}, \\
\left(d, \bar{\chi}\left|\left\{J^{i}, G^{j a}\right\}\right| c, \chi\right) & =\frac{1}{2} \delta_{i j} \delta_{\bar{\chi} \chi} \Lambda_{c}^{a, d}, \\
\left(d, \bar{\chi}\left|\left\{T^{a}, T^{b}\right\}\right| c, \chi\right) & =\delta_{\bar{\chi} \chi}\left(f^{a e d} f^{b e c}+f^{b e d} f^{a e c}\right),
\end{aligned}
$$




$$
\begin{aligned}
\left(d, \bar{\chi}\left|\left\{T^{a}, G^{i b}\right\}\right| c, \chi\right) & =\frac{1}{2} \sigma_{\bar{\chi} \chi}^{(i)}\left(i f^{a e d} \Lambda_{c}^{b, e}+\Lambda_{e}^{b, d} i f f^{a c e}\right), \\
\left(d, \bar{\chi}\left|\left\{G^{i a}, G^{j b}\right\}\right| c, \chi\right) & =\frac{1}{3} \delta_{i j} \delta_{\bar{\chi} \chi}\left(\frac{3}{4} \Lambda_{e}^{a, d} \Lambda_{c}^{b, e}+\frac{1}{4} \Lambda_{x y z}^{a, d} \Lambda_{c}^{b, x y z}+(a \leftrightarrow b)\right) \\
& +\frac{1}{2} i \varepsilon_{i j k} \sigma_{\bar{\chi} \chi}^{(k)}\left(\frac{1}{2} \Lambda_{e}^{a, d} \Lambda_{c}^{b, e}-\frac{1}{12} \Lambda_{x y z}^{a, d} \Lambda_{c}^{b, x y z}-(a \leftrightarrow b)\right) .
\end{aligned}
$$

Here, the spin-summation index $k$ takes the values $1,2,3$ and the flavour summation indices are $x, y, z=1,2,3$ and $e=1, \ldots 8$.

Operators evaluated between the decuplet states lead to

$$
\begin{aligned}
&\left(n o p, \bar{\chi}\left|\left\{J^{i}, J^{j}\right\}\right| k l m, \chi\right)=\frac{1}{3} \delta_{i j} \delta_{\bar{\chi} \chi} \frac{15}{2} \delta_{k l m}^{n o p} \\
&-\frac{1}{2}\left(S^{(i)} S^{(j) \dagger}+S^{(j)} S^{(i) \dagger}-\frac{2}{3} \delta_{i j}\right)_{\bar{\chi} \chi} 6 \delta_{k l m}^{n o p}, \\
&\left(n o p, \bar{\chi}\left|\left\{J^{i}, T^{a}\right\}\right| k l m, \chi\right)= \frac{9}{4}\left(\vec{S} \sigma^{(i)} \vec{S}^{\dagger}\right)_{\bar{\chi} \chi} \delta_{r s t}^{n o p} \Lambda_{k l m}^{a, r s t}, \\
&\left(n o p, \bar{\chi}\left|\left\{J^{i}, G^{j a}\right\}\right| k l m, \chi\right)= \frac{1}{3} \delta_{i j} \delta_{\bar{\chi} \chi} \frac{15}{4} \delta_{r s t}^{n o p} \Lambda_{k l m}^{a, r s t} \\
&- \frac{1}{2}\left(S^{(i)} S^{(j) \dagger}+S^{(j)} S^{(i) \dagger}-\frac{2}{3} \delta_{i j}\right)_{\bar{\chi} \chi} 3 \delta_{r s t}^{n o p} \Lambda_{k l m}^{a, r s t}, \\
&\left(n o p, \bar{\chi}\left|\left\{T^{a}, T^{b}\right\}\right| k l m, \chi\right)= \frac{9}{4} \delta_{\bar{\chi} \chi} \delta_{r s t}^{n o p}\left(\Lambda_{x y z}^{a, r s t} \Lambda_{k l m}^{b, x y z}+\Lambda_{x y z}^{b, r s t} \Lambda_{k l m}^{a, x y z}\right), \\
&\left(n o p, \bar{\chi}\left|\left\{T^{a}, G^{i b}\right\}\right| k l m, \chi\right)= \frac{9}{8}\left(\vec{S} \sigma^{(i)} \vec{S}^{\dagger}\right)_{\bar{\chi} \chi} \delta_{r s t}^{n o p}\left(\Lambda_{x y z}^{a, r s t} \Lambda_{k l m}^{b, x y z}+\Lambda_{x y z}^{b, r s t} \Lambda_{k l m}^{a, x y z}\right), \\
&\left(n o p, \bar{\chi}\left|\left\{G^{i a}, G^{j b}\right\}\right| k l m, \chi\right)= \frac{1}{3} \delta^{i j} \delta_{\bar{\chi} \chi}\left(\frac{1}{8} \Lambda_{c}^{a, n o p} \Lambda_{k l m}^{b, c}+\frac{15}{16} \delta_{r s t}^{n o p} \Lambda_{x y z}^{a, r s t} \Lambda_{k l m}^{b, x y z}+(a \leftrightarrow b)\right) \\
&+ \frac{1}{2} i \varepsilon_{i j k}\left(\vec{S} \sigma^{(k)} \vec{S}^{\dagger}\right)_{\bar{\chi} \chi} \\
& \times\left(\frac{1}{8} \Lambda_{c}^{a, n o p} \Lambda_{k l m}^{b, c}+\frac{3}{8} \delta_{r s t}^{n o p} \Lambda_{x y z}^{a, r s t} \Lambda_{k l m}^{b, x y z}-(a \leftrightarrow b)\right) \\
&+ \frac{1}{2}\left(S^{(i)} S^{(j) \dagger}+S^{(j)} S^{(i) \dagger}-\frac{2}{3} \delta_{\bar{\chi} \chi}^{i j}\right)_{\bar{\chi} \chi} \\
& \times\left(\frac{1}{8} \Lambda_{c}^{a, n o p} \Lambda_{k l m}^{b, c}-\frac{3}{4} \delta_{r s t}^{n o p} \Lambda_{x y z}^{a, r s t} \Lambda_{k l m}^{b, x y z}+(a \leftrightarrow b)\right),
\end{aligned}
$$

with the summation indices $k=1,2,3$ and $r, s, t, x, y, z=1,2,3$ and $c=1, \ldots 8$.

The transition matrix elements from a baryon-octet to a baryon-decuplet state vanish unless at least one spin-flavor operator $G^{i a}$ is involved. It holds:

$$
\begin{aligned}
\left(n o p, \bar{\chi}\left|\left\{J^{i}, G^{j a}\right\}\right| c, \chi\right) & =\frac{1}{2} i \varepsilon_{i j k} S_{\bar{\chi} \chi}^{(k)} \frac{3}{2 \sqrt{2}} \Lambda_{c}^{a, n o p} \\
& +\frac{1}{2}\left(S^{(i)} \sigma^{(j)}+S^{(j)} \sigma^{(i)}\right)_{\bar{\chi} \chi} \frac{1}{2 \sqrt{2}} \Lambda_{c}^{a, n o p} \\
\left(n o p, \bar{\chi}\left|\left\{T^{a}, G^{i b}\right\}\right| c, \chi\right) & =S_{\bar{\chi} \chi}^{(i)} \frac{1}{2 \sqrt{2}}\left(i f^{a b d} \Lambda_{c}^{d, n o p}+2 i f^{a c d} \Lambda_{d}^{b, n o p}\right),
\end{aligned}
$$




$$
\begin{aligned}
\left(n o p, \bar{\chi}\left|\left\{G^{i a}, G^{j b}\right\}\right| c, \chi\right)= & \frac{1}{2} i \varepsilon_{i j k} S_{\bar{\chi} \chi}^{(k)} \frac{1}{4 \sqrt{2}}\left(\frac{5}{3}\left(i f^{a b d} \Lambda_{c}^{d, n o p}+i f^{a c d} \Lambda_{d}^{b, n o p}\right)\right. \\
& \left.-\Lambda_{d}^{a, n o p} \Lambda_{c}^{b, d}-(a \leftrightarrow b)\right) \\
& +\frac{1}{2}\left(S^{(i)} \sigma^{(j)}+S^{(j)} \sigma^{(i)}\right)_{\bar{\chi} \chi} \frac{1}{4 \sqrt{2}}\left(\Lambda_{d}^{a, n o p}\left(d^{b c d}+i f^{b c d}\right)+(a \leftrightarrow b)\right),
\end{aligned}
$$

with the summation indices $k=1,2,3$ and $d=1, \cdots, 8$.

\section{E.5. Two-body operators, II}

Matrix elements of operators with two open adjoint flavour indices can be rewritten in a more convenient form, which makes the matching with the spin and flavour structures in the four-point interaction in the chiral Lagrangian more explicit. We recall first the possible flavour structures of the interaction terms given in Section 1.4.2 (see (C.8, C.18 C.23).

Combining four octets (baryon, anti-baryon and two meson octets) leads to the following combinations of Gell-Mann-matrices:

$$
\begin{aligned}
& \operatorname{tr}(\bar{B} B) \operatorname{tr}\left(\Phi_{1} \Phi_{2}\right)=\frac{1}{2} \bar{b}^{d} b^{c} \phi_{1}^{a} \phi_{2}^{b} F_{1 d c}^{a b(+)}, \\
& \operatorname{tr}\left(\bar{B} \Phi_{1}\right) \operatorname{tr}\left(\Phi_{2} B\right) \pm \operatorname{tr}\left(\bar{B} \Phi_{2}\right) \operatorname{tr}\left(\Phi_{1} B\right)=\frac{1}{2} \bar{b}^{d} b^{c} \phi_{1}^{a} \phi_{2}^{b} F_{2 d c}^{a b( \pm)} \\
& \operatorname{tr}\left(\bar{B}\left[\left[\Phi_{1}, \Phi_{2}\right]_{ \pm}, B\right]_{+}\right)=\frac{1}{2} \bar{b}^{d} b^{c} \phi_{1}^{a} \phi_{2}^{b} F_{3 d c}^{a b( \pm)}, \\
& \operatorname{tr}\left(\bar{B}\left[\left[\Phi_{1}, \Phi_{2}\right]_{ \pm}, B\right]_{-}\right)=\frac{1}{2} \bar{b}^{d} b^{c} \phi_{1}^{a} \phi_{2}^{b} F_{4 d c}^{a b( \pm)}, \\
& \operatorname{tr}\left(\bar{B} \Phi_{1} B \Phi_{2}\right)-\operatorname{tr}\left(\bar{B} \Phi_{2} B \Phi_{1}\right)=\frac{1}{2} \bar{b}^{d} b^{c} \phi_{1}^{a} \phi_{2}^{b} F_{5 d c}^{a b(-)},
\end{aligned}
$$

where the structures

$$
\begin{aligned}
& F_{1 d c}^{a b(+)}=4 \delta^{d c} \delta^{a b}, \\
& F_{2 d c}^{a b( \pm)}=4\left(\delta^{d a} \delta^{b c} \pm \delta^{d b} \delta^{a c}\right), \\
& F_{3 d c}^{a b( \pm)}=\operatorname{tr}\left(\lambda^{d}\left[\left[\lambda^{a}, \lambda^{b}\right]_{ \pm}, \lambda^{c}\right]_{+}\right)= \begin{cases}\frac{16}{3} \delta^{a b} \delta^{d c}+8 d^{a b e} d^{e c d} & \text { if }(+) \\
8 i f^{a b e} d^{e c d} & \text { if }(-),\end{cases} \\
& F_{4 d c}^{a b( \pm)}=\operatorname{tr}\left(\lambda^{d}\left[\left[\lambda^{a}, \lambda^{b}\right]_{ \pm}, \lambda^{c}\right]_{-}\right)= \begin{cases}8 i d^{a b e} f^{e c d} & \text { if }(+) \\
8 i f^{a b e} i f f^{e c d} & \text { if }(-),\end{cases} \\
& F_{5 d c}^{a b(-)}=\operatorname{tr}\left(\lambda^{d} \lambda^{a} \lambda^{c} \lambda^{b}\right)-\operatorname{tr}\left(\lambda^{d} \lambda^{b} \lambda^{c} \lambda^{a}\right)=-4 i\left(d^{a d e} f^{b c e}+f^{a d e} d^{b c e}\right),
\end{aligned}
$$

are introduced to simplify the notation in the following. The plus and minus signs in $F^{( \pm)}$denote the symmetry properties of the flavour structures concerning the interchange of two mesonic octets (interchange of $\lambda^{(a)}$ and $\lambda^{(b)}$ ). 
For the analogous interaction with the decuplet baryons one is confronted with the following flavour contractions:

$$
\begin{aligned}
\operatorname{tr}(\bar{\Delta} \cdot \Delta) \operatorname{tr}\left(\Phi_{1} \Phi_{2}\right) & =\bar{\Delta}_{\text {nop }} \Delta^{k l m} \phi_{1}^{a} \phi_{2}^{b} F_{1 \text { nop }, k l m}^{a b(+)}, \\
\operatorname{tr}\left((\bar{\Delta} \cdot \Delta)\left[\Phi_{1}, \Phi_{2}\right]_{ \pm}\right) & =\bar{\Delta}_{n o p} \Delta^{k l m} \phi_{1}^{a} \phi_{2}^{b} F_{2 \text { nop }, k l m}^{a b( \pm)}, \\
\operatorname{tr}\left(\left(\bar{\Delta} \cdot \Phi_{1}\right)\left(\Phi_{2} \cdot \Delta\right)\right)+\operatorname{tr}\left(\left(\bar{\Delta} \cdot \Phi_{2}\right)\left(\Phi_{1} \cdot \Delta\right)\right) & =\bar{\Delta}_{\text {nop }} \Delta^{k l m} \phi_{1}^{a} \phi_{2}^{b} F_{3 \text { nop }, k l m}^{a b(+)},
\end{aligned}
$$

with $^{3}$

$$
\begin{aligned}
F_{1 \text { nop }, k l m}^{a b(+)} & \equiv 2 \delta^{a b} \delta_{k l m}^{n o p}, \\
F_{2 \text { nop }, k l m}^{a b( \pm)} & \equiv \delta_{q r s}^{n o p} \delta_{k l m}^{\text {qrt }}\left(\lambda^{(a)} \lambda^{(b)} \pm \lambda^{(b)} \lambda^{(a)}\right)_{s t}= \begin{cases}\frac{4}{3} \delta^{a b} \delta_{k l m}^{n o p}+2 d^{a b e} \delta_{x y z}^{n o p} \Lambda_{k l m}^{e, x y z} & \text { if }(+) \\
2 i f^{a b e} \delta_{x y z}^{n o p} \Lambda_{k l m}^{e, x y z} & \text { if }(-),\end{cases} \\
F_{3 n o p, k l m}^{a b(+)} & \equiv \delta_{q r s}^{n o p} \delta_{k l m}^{v x s} \varepsilon_{r t u} \varepsilon_{x y u}\left(\lambda_{q t}^{(a)} \lambda_{y v}^{(b)}+\lambda_{q t}^{(b)} \lambda_{y v}^{(a)}\right) \\
& =2 \delta^{a b} \delta_{k l m}^{n o p}+3 d^{a b c} \delta_{r s t}^{n o p} \Lambda_{k l m}^{c, r s t}-\frac{3}{2} \delta_{r s t}^{n o p}\left(\Lambda_{x y z}^{a, r s t} \Lambda_{k l m}^{b, x y z}+\Lambda_{x y z}^{b, r s t} \Lambda_{k l m}^{a, x y z}\right) .
\end{aligned}
$$

The flavour structures of the interaction containing octet and decuplet baryons are:

$$
\begin{aligned}
\operatorname{tr}\left((\bar{\Delta} \cdot B)\left[\Phi_{1}, \Phi_{2}\right]_{ \pm}\right) & =\frac{1}{\sqrt{2}} \bar{\Delta}_{n o p} b^{c} \phi_{1}^{a} \phi_{2}^{b} F_{1 n o p, c}^{a b( \pm)} \\
\operatorname{tr}\left(\left(\bar{\Delta} \cdot \Phi_{1}\right)\left(\Phi_{2} \cdot B\right)\right) \pm \operatorname{tr}\left(\left(\bar{\Delta} \cdot \Phi_{2}\right)\left(\Phi_{1} \cdot B\right)\right) & =\frac{1}{\sqrt{2}} \bar{\Delta}_{n o p} b^{c} \phi_{1}^{a} \phi_{2}^{b} F_{2 n o p, c}^{a b( \pm)}
\end{aligned}
$$

with

$$
\begin{aligned}
F_{1 \text { nop }, c}^{a b( \pm)} & =\delta_{i j k}^{n o p} \lambda_{i q}^{(c)} \varepsilon_{j q r}\left(\left[\lambda^{(a)}, \lambda^{(b)}\right]_{ \pm}\right)_{k r}= \begin{cases}2 d^{a b e} \Lambda_{e}^{c, n o p} & \text { if }(+) \\
2 i f^{a b e} \Lambda_{e}^{c, n o p} & \text { if }(-),\end{cases} \\
F_{2 n o p, c}^{a b( \pm)} & =\delta_{i j k}^{n o p} \varepsilon_{j q r}\left(\lambda_{i q}^{(a)}\left(\lambda^{(b)} \lambda^{(c)}\right)_{k r} \pm \lambda_{i q}^{(b)}\left(\lambda^{(a)} \lambda^{(c)}\right)_{k r}\right) \\
& =\Lambda_{e}^{a, \text { nop }}\left(d^{b c e}+i f^{b c e}\right) \pm \Lambda_{e}^{b, n o p}\left(d^{a c e}+i f^{a c e}\right) .
\end{aligned}
$$

In the following, matrix elements of the large- $N_{c}$ operators with two open flavour indices are expressed as functions of the flavour structures introduced above. Furthermore, several combinations with definite spin-flavour multiplet structures (see the discussion leading to (B.20) for futher details)

$$
\begin{aligned}
& \mathcal{P}^{(1, \overline{10}+10)}\left\{T^{a}, G^{i b}\right\} \equiv\left\{T^{a}, G^{i b}\right\}-\left\{T^{b}, G^{i a}\right\}-\frac{2}{3} f^{a b c} f^{c g h}\left\{T^{g}, G^{i a h}\right\}, \\
& \mathcal{P}^{(1,27)}\left\{T^{a}, G^{i b}\right\} \equiv\left\{T^{a}, G^{i b}\right\}+\left\{T^{b}, G^{i a}\right\}-\frac{1}{4} \delta^{a b}\left\{T^{c}, G^{i c}\right\}-\frac{6}{5} d^{a b c} d^{c g h}\left\{T^{g}, G^{i h}\right\}, \\
& \text { etc. }
\end{aligned}
$$

\footnotetext{
${ }^{3}$ The obvious combination $F_{3 \text { nop } k l m}^{a b(-)}$ is redundant. See also footnote 3 on page 92 .
} 
are rewriten too. For the octet baryons one has:

$$
\begin{aligned}
\left(d, \bar{\chi}\left|d^{a b e} T^{e}\right| c, \chi\right) & =\delta_{\bar{\chi} \chi} \frac{1}{8} F_{4 d c}^{a b(+)}, \\
\left(d, \bar{\chi}\left|f^{a b e} T^{e}\right| c, \chi\right) & =\delta_{\bar{\chi} \chi}(-i) \frac{1}{8} F_{4 d c}^{a b(-)}, \\
\left(d, \bar{\chi}\left|d^{a b e} G^{i e}\right| c, \chi\right) & =\frac{1}{2} \sigma_{\bar{\chi} \chi}^{(i)}\left(-\frac{1}{6} F_{1 d c}^{a b(+)}+\frac{1}{8} F_{3 d c}^{a b(+)}+\frac{1}{12} F_{4 d c}^{a b(+)}\right) \\
\left(d, \bar{\chi}\left|f^{a b e} G^{i e}\right| c, \chi\right) & =\frac{1}{2} \sigma_{\bar{\chi} \chi}^{(i)}\left(-\frac{i}{8} F_{3 d c}^{a b(-)}-\frac{i}{12} F_{4 d c}^{a b(-)}\right), \\
\left(d, \bar{\chi}\left|\left\{T^{a}, T^{b}\right\}\right| c, \chi\right) & =\delta_{\bar{\chi} \chi} \frac{1}{4}\left(-F_{1 d c}^{a b(+)}-F_{2 d c}^{a b(+)}+\frac{3}{2} F_{3 d c}^{a b(+)}\right), \\
\left(d, \bar{\chi}\left|\left\{T^{a}, G^{i b}\right\}\right| c, \chi\right) & =\frac{1}{2} \sigma_{\bar{\chi} \chi}^{(i)} \frac{1}{6}\left(-F_{1 d c}^{a b(+)}-F_{2 d c}^{a b(+)}+\frac{3}{2} F_{3 d c}^{a b(+)}+\frac{3}{4} F_{4 d c}^{a b(+)}+\frac{3}{2} F_{5 d c}^{a b(-)}\right), \\
\left(d, \bar{\chi}\left|\left\{G^{i a}, G^{j b}\right\}\right| c, \chi\right) & =\frac{1}{3} \delta_{i j} \delta_{\bar{\chi} \chi} \frac{1}{16}\left(7 F_{1 d c}^{a b(+)}-F_{2 d c}^{a b(+)}-\frac{3}{2} F_{3 d c}^{a b(+)}+4 F_{4 d c}^{a b(+)}\right) \\
& +\frac{1}{2} i \varepsilon_{i j k} \sigma_{\bar{\chi} \chi}^{(k)} \frac{1}{8}\left(-F_{2 d c}^{a b(-)}+F_{3 d c}^{a b(-)}+\frac{5}{6} F_{4 d c}^{a b(-)}\right)
\end{aligned}
$$

and

$$
\begin{aligned}
\mathcal{P}^{(0,27)}\left(d, \bar{\chi}\left|\left\{T^{a}, T^{b}\right\}\right| c, \chi\right) & =\delta_{\bar{\chi} \chi} \frac{1}{10}\left(-\frac{11}{4} F_{1 d c}^{a b(+)}-5 F_{2 d c}^{a b(+)}+3 F_{3 d c}^{a b(+)}\right), \\
\mathcal{P}^{(1, \overline{10}+10)}\left(d, \bar{\chi}\left|\left\{T^{g}, G^{i h}\right\}\right| c, \chi\right) & =\frac{1}{2} \sigma_{\bar{\chi} \chi}^{(i)} \frac{1}{2} F_{5 d c}^{a b(-)}, \\
f^{a e g} d^{b e h}\left(d, \bar{\chi}\left|\left\{T^{g}, G^{i h}\right\}\right| c, \chi\right) & =\frac{1}{2} \sigma_{\bar{\chi} \chi}^{(i)}\left(-\frac{i}{4} F_{2 d c}^{a b(-)}-\frac{i}{8} F_{3 d c}^{a b(-)}-\frac{i}{48} F_{4 d c}^{a b(-)}\right), \\
\mathcal{P}^{(1, \overline{10}+10)}\left(d, \bar{\chi}\left|f^{a e g} d^{b e h}\left\{T^{g}, G^{i h}\right\}\right| c, \chi\right) & =\frac{1}{2} \sigma_{\bar{\chi} \chi}^{(i)}\left(-\frac{i}{2} F_{2 d c}^{a b(-)}+\frac{i}{6} F_{4 d c}^{a b(-)}\right), \\
\mathcal{P}^{(1,27)}\left(d, \bar{\chi}\left|\left\{T^{a}, G^{i b}\right\}\right| c, \chi\right) & =\frac{1}{2} \sigma_{\bar{\chi} \chi}^{(i)}\left(-\frac{11}{60} F_{1 d c}^{a b(+)}-\frac{1}{3} F_{2 d c}^{a b(+)}+\frac{1}{5} F_{3 d c}^{a b(+)}\right) .
\end{aligned}
$$

Evaluation of the operators between the decuplet states leads to:

$$
\begin{aligned}
& \left(\text { nop }, \bar{\chi}\left|d^{a b e} T^{e}\right| k l m, \chi\right)=\delta_{\bar{\chi} \chi}\left(-\frac{1}{2} F_{1 \text { nop }, k l m}^{a b(+)}+\frac{3}{4} F_{2 \text { nop }, k l m}^{a b(+)}\right), \\
& \left(\text { nop, } \bar{\chi}\left|f^{a b e} T^{e}\right| k l m, \chi\right)=\delta_{\bar{\chi} \chi}(-i) \frac{3}{4} F_{2 \text { nop }, k l m}^{a b(-)} \text {, } \\
& \left(n o p, \bar{\chi}\left|d^{a b e} G^{i e}\right| k l m, \chi\right)=\frac{1}{2}\left(\vec{S} \sigma^{(i)} \vec{S}^{\dagger}\right)_{\bar{\chi} \chi}\left(-\frac{1}{2} F_{1 \text { nop }, k l m}^{a b(+)}+\frac{3}{4} F_{2 \text { nop }, k l m}^{a b(+)}\right) \text {, } \\
& \left(n o p, \bar{\chi}\left|f^{a b e} G^{i e}\right| i j k, \chi\right)=\frac{1}{2}\left(\vec{S} \sigma^{(i)} \vec{S}^{\dagger}\right)_{\bar{\chi} \chi}(-i) \frac{3}{4} F_{2 n o p, k l m}^{a b(-)} \text {, } \\
& \left(\text { nop, } \bar{\chi}\left|\left\{T^{a}, T^{b}\right\}\right| k l m, \chi\right)=\delta_{\bar{\chi} \chi}\left(\frac{9}{4} F_{2 \text { nop }, k l m}^{a b(+)}-\frac{3}{2} F_{3 \text { nop }, k l m}^{a b(+)}\right), \\
& \left(\text { nop, } \bar{\chi}\left|\left\{T^{a}, G^{i b}\right\}\right| k l m, \chi\right)=\frac{1}{2}\left(\vec{S} \sigma^{(i)} \vec{S}^{\dagger}\right)_{\bar{\chi} \chi}\left(\frac{9}{4} F_{2 \text { nop }, k l m}^{a b(+)}-\frac{3}{2} F_{3 n o p, k l m}^{a b(+)}\right) \text {, } \\
& \left(n o p, \bar{\chi}\left|\left\{G^{i a}, G^{j b}\right\}\right| k l m, \chi\right)=\frac{1}{3} \delta^{i j} \delta_{\bar{\chi} \chi} \frac{15}{16}\left(F_{2 n o p, k l m}^{a b(+)}-\frac{2}{5} F_{3 n o p, k l m}^{a b(+)}\right) \\
& +\frac{1}{2} i \varepsilon_{i j k}\left(\vec{S} \sigma^{(k)} \vec{S}^{\dagger}\right)_{\bar{\chi} \chi} \frac{3}{8} F_{2 n o p, k l m}^{a b(-)}
\end{aligned}
$$




$$
+\frac{1}{2}\left(S^{(i)} S^{(j) \dagger}+S^{(j)} S^{(i) \dagger}-\frac{2}{3} \delta^{i j}\right)_{\bar{\chi} \chi} \frac{3}{4}\left(-F_{2 \text { nop }, k l m}^{a b(+)}+F_{3 n o p, k l m}^{a b(+)}\right),
$$

and

$$
\begin{aligned}
\mathcal{P}^{(0,27)}\left(n o p, \bar{\chi}\left|\left\{T^{a}, T^{b}\right\}\right| k l m, \chi\right) & =\delta_{\bar{\chi} \chi} \frac{3}{10}\left(F_{1 \text { nop,ijk }}^{a b(+)}+6 F_{2 \text { nop }, i j k}^{a b(+)}-10 F_{3 n o p, i j k}^{a b(+)}\right), \\
\mathcal{P}^{(1, \overline{10}+10)}\left(n o p, \bar{\chi}\left|\left\{T^{g}, G^{i h}\right\}\right| k l m, \chi\right) & =0 \\
f^{a e g} d^{b e h}\left(n o p, \bar{\chi}\left|\left\{T^{g}, G^{i h}\right\}\right| k l m, \chi\right) & =\frac{1}{2}\left(\vec{S} \sigma^{(i)} \vec{S}^{\dagger}\right)_{\bar{\chi} \chi}(-i) \frac{9}{8} F_{2 n o p, k l m}^{a b(-)}, \\
\mathcal{P}^{(1, \overline{10}+10)} f^{a e g} d^{b e h}\left(n o p, \bar{\chi}\left|\left\{T^{g}, G^{i h}\right\}\right| k l m, \chi\right) & =0 \\
\mathcal{P}^{(1,27)}\left(n o p, \bar{\chi}\left|\left\{T^{g}, G^{i h}\right\}\right| k l m, \chi\right) & =\frac{1}{2}\left(\vec{S} \sigma^{(i)} \vec{S}^{\dagger}\right)_{\bar{\chi} \chi} \\
& \times \frac{3}{10}\left(F_{1 n o p, i j k}^{a b(+)}+6 F_{2 n o p, k l m}^{a b(+)}-10 F_{3 n o p, k l m}^{a b(+)}\right) \\
\mathcal{P}^{(2,27)}\left(n o p, \bar{\chi}\left|\left\{G^{i a}, G^{j b}\right\}\right| k l m, \chi\right) & =\frac{1}{2}\left(S^{(i)} S^{(j) \dagger}+S^{(j)} S^{(i) \dagger}-\frac{2}{3} \delta^{i j}\right)_{\bar{\chi} \chi} \\
& \times \frac{3}{2}\left(-\frac{1}{10} F_{1 n o p, k l m}^{a b(+)}-\frac{3}{5} F_{2 n o p, k l m}^{a b(+)}+F_{3 n o p, k l m}^{a b(+)}\right) .
\end{aligned}
$$

Finally, the non-vanishing octet-decuplet transition matrix elements are given by:

$$
\begin{aligned}
\left(n o p, \bar{\chi}\left|d^{a b e} G^{i e}\right| c, \chi\right) & =S_{\bar{\chi} \chi}^{(i)} \frac{-1}{4 \sqrt{2}} F_{1 \text { nop }, c}^{a b(+)} \\
\left(n o p, \bar{\chi}\left|f^{a b e} G^{i e}\right| c, \chi\right) & =S_{\bar{\chi} \chi}^{(i)} \frac{i}{4 \sqrt{2}} F_{1 n o p, c}^{a b(-)}, \\
\left(n o p, \bar{\chi}\left|\left\{T^{a}, G^{i b}\right\}\right| c, \chi\right) & =S_{\bar{\chi} \chi}^{(i)} \frac{1}{4 \sqrt{2}}\left(-F_{1 \text { nop }, c}^{a b(+)}+F_{2 n o p, c}^{a b(+)}-3 F_{2 n o p, c}^{a b(-)}\right), \\
\left(n o p, \bar{\chi}\left|\left\{G^{i a}, G^{j b}\right\}\right| c, \chi\right) & =\frac{1}{2} i \varepsilon_{i j k} S^{(k)} \frac{1}{4 \sqrt{2}}\left(-F_{1 n o p, c}^{a b(-)}-3 F_{2 n o p, c}^{a b(-)}\right) \\
& +\frac{1}{2}\left(S^{(i)} \sigma^{(j)}+S^{(j)} \sigma^{(i)}\right) \frac{1}{4 \sqrt{2}} F_{2 n o p, c}^{a b(+)},
\end{aligned}
$$

and

$$
\begin{aligned}
\mathcal{P}^{(1, \overline{10}+10)}\left(n o p, \bar{\chi}\left|\left\{T^{a}, G^{i b}\right\}\right| c, \chi\right) & =S_{\bar{\chi} \chi}^{(i)} \frac{1}{2 \sqrt{2}}\left(F_{1 \text { nop }, c}^{a b(-)}-3 F_{2 n o p, c}^{a b(-)}\right) \\
f^{a e g} d^{b e h}\left(n o p, \bar{\chi}\left|\left\{T^{g}, G^{i h}\right\}\right| c, \chi\right) & =S_{\bar{\chi} \chi}^{(i)} \frac{i}{8 \sqrt{2}}\left(3 F_{1 n o p, c}^{a b(+)}+4 F_{1 n o p, c}^{a b(-)}-6 F_{2 n o p, c}^{a b(-)}\right) \\
\mathcal{P}^{(1, \overline{10}+10)} f^{a e g} d^{b e h}\left(n o p, \bar{\chi}\left|\left\{T^{g}, G^{i h}\right\}\right| c, \chi\right) & =S_{\bar{\chi} \chi}^{(i)} \frac{i}{2 \sqrt{2}}\left(F_{1 n o p, c}^{a b(-)}-3 F_{2 n o p, c}^{a b(-)}\right) \\
\mathcal{P}^{(1,27)}\left(n o p, \bar{\chi}\left|\left\{T^{g}, G^{i h}\right\}\right| c, \chi\right) & =S_{\bar{\chi} \chi}^{(i)} \frac{1}{10 \sqrt{2}}\left(F_{1 n o p, c}^{a b(+)}+5 F_{2 n o p, c}^{a b(+)}\right) \\
\mathcal{P}^{(2,27)}\left(n o p, \bar{\chi}\left|\left\{G^{i a}, G^{j b}\right\}\right| c, \chi\right) & =\frac{1}{2}\left(S^{(i)} \sigma^{(j)}+S^{(j)} \sigma^{(i)}\right) \frac{1}{10 \sqrt{2}}\left(F_{1 n o p, c}^{a b(+)}+5 F_{2 n o p, c}^{a b(+)}\right)
\end{aligned}
$$




\section{Appendix F.}

\section{Pauli- and spin transition matrices}

In this chapter some useful relations for the sigma- and "spin- $1 / 2$ to spin-3/2"-transition matrices are collected. These relations are frequently used in the course of the evaluation of the matrix elements in Appendix E.

Main properties of the Pauli-matrices

$$
\sigma_{1}=\left(\begin{array}{cc}
0 & 1 \\
1 & 0
\end{array}\right), \quad \sigma_{2}=\left(\begin{array}{cc}
0 & -i \\
i & 0
\end{array}\right), \quad \sigma_{3}=\left(\begin{array}{cc}
1 & 0 \\
0 & -1
\end{array}\right)
$$

are

$$
\begin{gathered}
\sigma_{i}^{\dagger}=\sigma_{i}, \quad \operatorname{tr}\left(\sigma_{i}\right)=0, \quad \operatorname{det} \sigma_{i}=-1, \quad \sigma_{i}^{T}=\sigma_{i}^{*}=-\sigma_{2} \sigma_{i} \sigma_{2} \\
{\left[\sigma_{i}, \sigma_{j}\right]=2 i \varepsilon_{i j k} \sigma_{k}, \quad\left\{\sigma_{i}, \sigma_{j}\right\}=2 \delta_{i j} .}
\end{gathered}
$$

Several relations can be obtained from the above identities:

$$
\begin{gathered}
\sigma_{i} \sigma_{j}=\delta_{i j}+i \varepsilon_{i j k} \sigma^{k}, \quad \sigma_{i} \sigma_{j} \sigma_{k}=i \varepsilon_{i j k}+\delta_{i j} \sigma_{k}-\delta_{i k} \sigma_{j}+\delta_{j k} \sigma_{i}, \\
\operatorname{tr}\left(\sigma_{i} \sigma_{j}\right)=2 \delta_{i j}, \quad \operatorname{tr}\left(\sigma_{i} \sigma_{j} \sigma_{k}\right)=2 i \varepsilon_{i j k}, \quad \sigma_{i} \sigma_{i}=3 \mathbb{1}_{(2 \times 2)}, \\
\sigma_{j} \sigma_{i} \sigma_{j}=-\sigma_{i}, \quad \epsilon_{i j k} \sigma_{i} \sigma_{j}=2 i \sigma_{k}, \quad \sigma_{j} \sigma_{i} \sigma_{k} \sigma_{j} \sigma_{i}=5 \sigma_{k} .
\end{gathered}
$$

The "spin-transition" matrices are given by ${ }^{1}$

$$
S_{1}=\left(\begin{array}{cc}
-\frac{1}{\sqrt{2}} & 0 \\
0 & -\frac{1}{\sqrt{6}} \\
\frac{1}{\sqrt{6}} & 0 \\
0 & \frac{1}{\sqrt{2}}
\end{array}\right), \quad S_{2}=\left(\begin{array}{cc}
\frac{i}{\sqrt{2}} & 0 \\
0 & \frac{i}{\sqrt{6}} \\
\frac{i}{\sqrt{6}} & 0 \\
0 & \frac{i}{\sqrt{2}}
\end{array}\right), \quad S_{3}=\left(\begin{array}{cc}
0 & 0 \\
\sqrt{\frac{2}{3}} & 0 \\
0 & \sqrt{\frac{2}{3}} \\
0 & 0
\end{array}\right),
$$

with the main properties

$$
\begin{gathered}
S_{i}^{\dagger} S_{j}=\delta_{i j}-\frac{1}{3} \sigma_{i} \sigma_{j}=\delta_{i j}-\frac{1}{3}\left(\delta_{i j}+i \varepsilon_{i j k} \sigma_{k}\right)=\frac{2}{3}\left(\delta_{i j}-i \varepsilon_{i j k} \frac{1}{2} \sigma_{k}\right), \\
\vec{S}^{\dagger} \cdot \vec{S}=2 \mathbb{1}_{(2 \times 2)}, \quad \vec{S} \cdot \vec{S}^{\dagger}=\mathbb{1}_{(4 \times 4)}, \quad \vec{\sigma} \cdot \vec{S}^{\dagger}=0, \quad \vec{S} \cdot \vec{\sigma}=0, \\
S_{i} \sigma_{j}-S_{j} \sigma_{i}=-i \varepsilon_{i j k} S_{k} .
\end{gathered}
$$

${ }^{1}$ The origin of the spin-transition matrices is discussed in Appendix H. 


$$
\begin{gathered}
S_{i} \sigma_{k} \sigma_{i}=2 S_{k}, \quad i \varepsilon_{i j k} S_{i} \sigma_{j}=\sigma_{k}, \\
\varepsilon_{i j k} S_{i} S_{j}^{\dagger}=i \vec{S} \sigma_{k} \vec{S}^{\dagger}, \quad \varepsilon_{i j k}\left(\vec{S} \sigma_{i} \vec{S}^{\dagger}\right)\left(\vec{S} \sigma_{j} \vec{S}^{\dagger}\right)=\frac{2}{3} \varepsilon_{i j k} S_{i} S_{j}^{\dagger}=\frac{2}{3} i \vec{S} \sigma_{k} \vec{S}^{\dagger}, \\
\left(\vec{S} \sigma_{k}^{*} \vec{S}^{\dagger}\right)=\left(\vec{S} \sigma_{k} \vec{S}^{\dagger}\right)^{T}, \quad \sum_{i}\left(\vec{S} \sigma^{(i)} \vec{S}^{\dagger}\right)\left(\vec{S} \sigma^{(i)} \vec{S}^{\dagger}\right)=\frac{5}{3} \mathbb{1}_{(4 \times 4)} .
\end{gathered}
$$

In the course of evaluation of the matrix elements of the spin-flavour operators

$$
\left\{J^{i}, J^{j}\right\}, \quad\left\{J^{i}, G^{j a}\right\} \quad\left\{G^{i a}, G^{j b}\right\}
$$

in Section E.4, one is confronted with several products of $\sigma$ and $S$ with two open vector indices. These products are reducible and can be decomposed into invariant, symmetric and antisymmetric components which transform independently of each other under $S O(3)$ rotations.

In general, a product of two vectors, $x_{i} y_{j}$, decomposes as follows:

$$
\begin{aligned}
x_{i} y_{j} & =\frac{1}{3} \delta_{i j} \vec{x} \cdot \vec{y}+\frac{1}{2}\left(x_{i} y_{j}-x_{j} y_{i}\right)+\frac{1}{2}\left(x_{i} y_{j}+x_{j} y_{i}-\frac{2}{3} \delta_{i j} \vec{x} \cdot \vec{y}\right) \\
& =\frac{1}{3} \delta_{i j} \vec{x} \cdot \vec{y}+\frac{1}{2} \epsilon_{i j k} \epsilon_{k l m} x_{l} y_{m}+\frac{1}{2}\left(x_{i} y_{j}+x_{j} y_{i}-\frac{2}{3} \delta_{i j} \vec{x} \cdot \vec{y}\right) .
\end{aligned}
$$

Having this and the above properties of $\sigma$ and $S$, the spin-part of the matrix elements of the operators in (F.7) can be rewritten using following decompositions:

$$
\begin{aligned}
\sigma_{i} \sigma_{j} & =\delta_{i j}+i \varepsilon_{i j k} \sigma_{k} \\
S_{i} S_{j}^{\dagger} & =\frac{1}{3} \delta_{i j} \mathbb{1}_{(4 \times 4)}+\frac{1}{2} i \varepsilon_{i j k}\left(\vec{S} \sigma_{k} \vec{S}^{\dagger}\right)+\frac{1}{2}\left(S_{i} S_{j}^{\dagger}+S_{j} S_{i}^{\dagger}-\frac{2}{3} \delta_{i j} \mathbb{1}_{(4 \times 4)}\right) \\
\left(\vec{S} \sigma_{i} \vec{S}^{\dagger}\right)\left(\vec{S} \sigma_{j} \vec{S}^{\dagger}\right) & =\frac{5}{9} \delta_{i j} \mathbb{1}_{(4 \times 4)}+\frac{1}{3} i \varepsilon_{i j k}\left(\vec{S} \sigma_{k} \vec{S}^{\dagger}\right)-\frac{2}{3}\left(S_{i} S_{j}^{\dagger}+S_{j} S_{i}^{\dagger}-\frac{2}{3} \delta_{i j} \mathbb{1}_{(4 \times 4)}\right) \\
S_{i} \sigma_{j} & =-\frac{1}{2} i \varepsilon_{i j k} S_{k}+\frac{1}{2}\left(S_{i} \sigma_{j}+S_{j} \sigma_{i}\right) \\
\left(\vec{S} \sigma_{i} \vec{S}^{\dagger}\right) S_{j} & =\frac{5}{6} i \varepsilon_{i j k} S_{k}+\frac{1}{6}\left(S_{i} \sigma_{j}+S_{j} \sigma_{i}\right) .
\end{aligned}
$$

In the third identity, the symmetric traceless component in $\left(\vec{S} \sigma_{i} \vec{S}^{\dagger}\right)\left(\vec{S} \sigma_{j} \vec{S}^{\dagger}\right)$ was simplified with the help of

$$
\begin{array}{r}
\left(\vec{S} \sigma_{i} \vec{S}^{\dagger}\right)\left(\vec{S} \sigma_{j} \vec{S}^{\dagger}\right)+\left(\vec{S} \sigma_{j} \vec{S}^{\dagger}\right)\left(\vec{S} \sigma_{i} \vec{S}^{\dagger}\right)-\frac{2}{3} \delta_{i j}\left(\vec{S} \sigma_{k} \vec{S}^{\dagger}\right)\left(\vec{S} \sigma_{k} \vec{S}^{\dagger}\right) \\
=-\frac{4}{3}\left(S_{i} S_{j}^{\dagger}+S_{j} S_{i}^{\dagger}-\frac{2}{3} \delta_{i j} S_{k} S_{k}^{\dagger}\right)
\end{array}
$$




\section{Appendix G.}

\section{Operator Identities}

The one-body operators in (2.24) are used to organize the $1 / N_{c}$ expansion of baryonic matrix elements of quark operators. The operator reduction rule, stated in Section 2.3.2 helps to eliminate the irrelevant combinations of these one-body operators. This reduction rule is based on group theoretical relations between the one body operators. The derivation of these operator identities was done in [22] and is briefly reviewed in this chapter. In the second part of this chapter the application of this rule is done for all possible products of two one-body operators in an explicit and detailed form. Furthermore, in the last part the (ir)relevance of contractions of flavour indices on the one-body operators with certain combinations of $f$ and $d$ symbols of $S U(3)$ is discussed.

The identities in Table G.1 are collected in three different blocks according to their group theoretical structure. To discuss the derivation and the structure of these identities it is advantageous to use a uniformly normalised set of the $S U(6)$-generators:

$$
q^{\dagger} \Lambda^{A} q=\left\{J^{i} / \sqrt{3}, T^{a} / \sqrt{2}, \sqrt{2} G^{i a}\right\}, \quad A=1, \ldots 35,
$$

with

$$
\operatorname{tr}\left(q^{\dagger} \Lambda^{A} q q^{\dagger} \Lambda^{B} q\right)=\frac{1}{2} \delta^{A B}
$$

The first identity in Table G.1 relates two-body operators to the zero-body operator $\mathbb{1}$, the $S U(6)$ singlet. This singlet operator is nothing else but the quadratic Casimir operator $C_{2}$ of the symmetry group:

$$
q^{\dagger} \Lambda^{A} q q^{\dagger} \Lambda^{A} q=\frac{1}{2}\left\{q^{\dagger} \Lambda^{A} q, q^{\dagger} \Lambda^{A} q\right\}=C_{2} \mathbb{1} .
$$

The identities in the second block in Table G.1 relate two-body operators to one-body operators. These identities result from the cubic Casimir relations

$$
d_{A B C} q^{\dagger} \Lambda^{A} q q^{\dagger} \Lambda^{B} q q^{\dagger} \Lambda^{C} q=\frac{1}{2} d_{A B C} q^{\dagger} \Lambda^{A} q\left\{q^{\dagger} \Lambda^{B} q, q^{\dagger} \Lambda^{C} q\right\}=C_{3} \mathbb{1} .
$$

Based on this relation one derives ${ }^{1}$

$$
d^{A B C}\left\{q^{\dagger} \Lambda^{B} q, q^{\dagger} \Lambda^{C} q\right\}=2 \frac{C_{3}}{C_{2}} q^{\dagger} \Lambda^{A} q
$$

\footnotetext{
${ }^{1}$ The identity between (G.4) and (G.5) is shown by multiplying both sides in (G.5) by $q^{\dagger} \Lambda^{A} q$, summing over $A$ and using the relation for the quadratic Casimir operator.
} 


\begin{tabular}{|c|c|}
\hline $2\left\{J^{i}, J^{i}\right\}+3\left\{T^{a}, T^{a}\right\}+12\left\{G^{i a}, G^{i a}\right\}=5\left(N_{c}+6\right) \mathbb{1}$ & $(0,0)$ \\
\hline $\begin{array}{c}d^{a b c}\left\{G^{i a}, G^{i b}\right\}+\frac{2}{3}\left\{J^{i}, G^{i c}\right\}+\frac{1}{4} d^{a b c}\left\{T^{a}, T^{b}\right\}=\frac{2}{3}\left(N_{c}+3\right) T^{c} \\
\left\{T^{a}, G^{i a}\right\}=\frac{2}{3}\left(N_{c}+3\right) J^{i} \\
\frac{1}{3}\left\{J^{k}, T^{c}\right\}+d^{a b c}\left\{T^{a}, G^{k b}\right\}-\varepsilon^{i j k} f^{a b c}\left\{G^{i a}, G^{j b}\right\}=\frac{4}{3}\left(N_{c}+3\right) G^{k c}\end{array}$ & $\begin{array}{l}(0,8) \\
(1,0) \\
(1,8)\end{array}$ \\
\hline 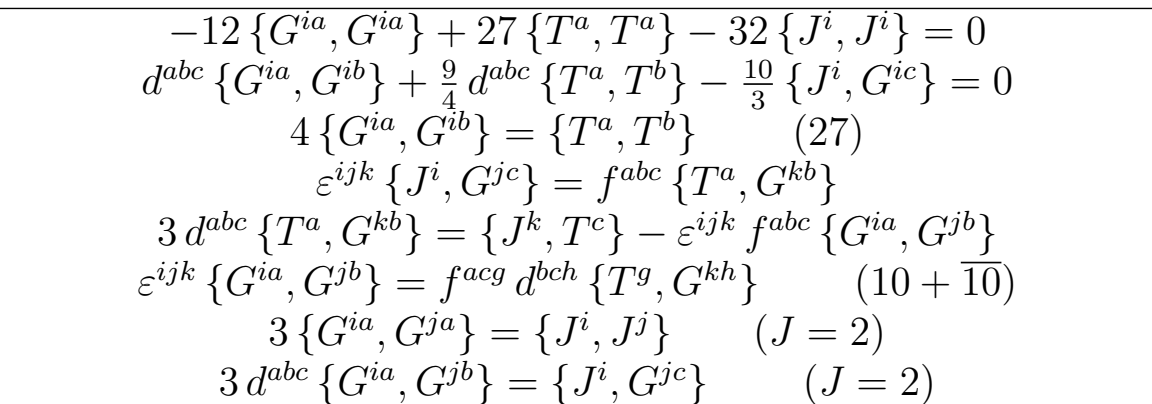 & $\begin{array}{c}(0,0) \\
(0,8) \\
(0,27) \\
(1,8) \\
(1,8) \\
(1,10+\overline{10}) \\
(2,0) \\
(2,8)\end{array}$ \\
\hline
\end{tabular}

Table G.1.: Operator identities for $N_{F}=3$. The second column gives the transformation properties of the expressions under $S U(2)_{\text {spin }} \otimes S U(3)_{\text {flavour }}$ symmetry.

The constants $C_{2}$ and $C_{3}$ for the completely symmetric $S U(6)$ baryon states were evaluated in [22]. One obtains:

$$
\begin{aligned}
\left\{q^{\dagger} \Lambda^{A} q, q^{\dagger} \Lambda^{A} q\right\} & =N_{c}\left(N_{c}+2 N_{F}\right)\left(1-1 / 2 N_{F}\right) \mathbb{1}, \\
d^{A B C}\left\{q^{\dagger} \Lambda^{B} q, q^{\dagger} \Lambda^{C} q\right\} & =2\left(N_{c}+N_{F}\right)\left(1-1 / N_{F}\right)\left\{q^{\dagger} \Lambda^{A} q\right\} .
\end{aligned}
$$

The product of two operators on the r.h.s of the second line in the above expression can be decomposed into three different components (flavour index only, spin index only and spin-flavour index) which transform according to different irreducible representations of $S U(2) \otimes S U(3)$. The three lines in the second block in Table G.1 correspond to this decomposition.

Finally, the identities in the third block are based on the observation, that antisymmetric combinations in the products of two one-body operators vanish when acting on totally symmetric baryonic states. The identities in the last block are obtained by projecting on antisymmetric parts in all possible products and setting them equal to zero.

A quark operator transforms as a 6 . Accordingly, the product of two quark operators decomposes to

$$
6 \otimes 6=21 \oplus \mathbf{1 5} .
$$

Due to the bosonic algebra of the spin-flavour quark operators, only the symmetric component, 21, survives in this decomposition. Therefore, the components in a normal ordered symmetric two-body operator are given by

$$
\overline{\mathbf{2 1}} \otimes \mathbf{2 1}=\mathbf{1} \oplus \mathbf{3 5} \oplus \mathbf{4 0 5} .
$$

On the other hand, the symmetric components in the product of two one-body operators, each of them transforming as the $S U(6)$-adjoint representation, $\mathbf{3 5}$, are:

$$
(35 \otimes 35)_{S}=1 \oplus 35 \oplus 189 \oplus 405 .
$$




\begin{tabular}{|c|c|c|}
\hline$\left\{J^{i}, J^{j}\right\}$ & $(0,0)(2,0)$ & $(0,0)(2,0)$ \\
$\left\{J^{i}, T^{a}\right\}$ & $(1,8)$ & $(1,8)$ \\
$\left\{J^{i}, G^{j a}\right\}$ & $(0,8)(1,8)(2,8)$ & $(0,8)(1,8)(2,8)$ \\
$\left\{T^{a}, T^{b}\right\}$ & $(0,0)\left(0,8_{S}\right)(0,27)$ & $(0,27)$ \\
$\left\{T^{a}, G^{i b}\right\}$ & $(1,0)\left(1,8_{S}\right)\left(1,8_{A}\right)(1,10+10)(1,27)$ & $(1,10+10)(1,27)$ \\
$\left\{G^{i a}, G^{j b}\right\}$ & $(0,0)\left(0,8_{S}\right)(0,27)\left(1,8_{A}\right)(1,10+10)$ & $(2,27)$ \\
& $(2,0)\left(2,8_{S}\right)(2,27)$ & \\
\hline
\end{tabular}

Table G.2.: Symmetric products of two one-body quark operators and their decomposition into the $S U(2)_{\text {spin }} \otimes S U(3)_{\text {flavour }}$ representations. The 3 rd column contains only the relevant components in these products.

It is the 189-dimensional representation in (G.9) that is missing in (G.8) ${ }^{2}$. Building of all possible products of two one-body operators, projecting onto the 189-plet and setting it equal to zero leads to the identities in the 3rd block in Table G.1.

In Table G.2 all possible symmetric products of two one-body operators are collected. Usually, a product of two operators contains components that transform according to different irreducible representation of $S U(2) \otimes S U(3)$. In total there are 22 different components, which are shown in the second column of that table. Twelve operator identities, listed in Table G.1, reduce the number of independent products to ten. Promoting the components in the 3rd column to be "relevant" allows to formulate the operator reduction rule, which was stated in Section 2.3.2. The results of this section are summarised in Tables G.1 and G.2. The operator reduction rule is another way to give the summary in a short form.

${ }^{2}$ The decomposition of the $S U(6)$ irreducible representations into $S U(2) \times S U(3)$ representations is:

$$
\begin{aligned}
1 & \rightarrow(0,0) \\
35 & \rightarrow(0,8),(1,0),(1,8) \\
189 & \rightarrow(0,0),(0,8),(0,27),(1,8),(1,8),(1, \overline{10}+10),(2,0),(2,8), \\
405 & \rightarrow(0,0),(0,8),(0,27),(1,8),(1,8),(1,27),(1, \overline{10}+10),(2,0),(2,8),(2,27) .
\end{aligned}
$$


In the following, the decomposition of all symmetric products of two one-body operators into their different spin-flavour multiplets is carried out with the help of the projectors given in Appendix B. The redundancy of those parts, which are not mentioned in the 3rd column of the Table G.2, is shown explicitly by applying the operator identities in Table G.1.

None of the parts in the products containing the $J$-operator is redundant ${ }^{3}$ :

$$
\begin{aligned}
\left\{J^{i}, J^{j}\right\} & =\frac{1}{3} \delta^{i j}\left\{J^{k}, J^{k}\right\}+\left(\left\{J^{i}, J^{j}\right\}-\frac{1}{3} \delta^{i j}\left\{J^{k}, J^{k}\right\}\right), \\
\left\{J^{i}, T^{a}\right\}, & \\
\epsilon_{i j k}\left\{J^{i}, G^{j a}\right\}, & \\
\left\{J^{i}, G^{j a}\right\} & =\frac{1}{3} \delta^{i j}\left\{J^{k}, G^{k a}\right\}+\frac{1}{2} \epsilon^{i j k} \epsilon^{k l m}\left\{J^{l}, G^{m a}\right\} \\
& +\frac{1}{2}\left(\left\{J^{i}, G^{j a}\right\}+\left\{J^{j}, G^{i a}\right\}-\frac{2}{3} \delta^{i j}\left\{J^{k}, G^{k a}\right\}\right) .
\end{aligned}
$$

Anticommutators with the $T$-operator are given by

$$
\begin{aligned}
\left\{T^{a}, T^{b}\right\} & =\frac{1}{8} \delta^{a b}\left\{T^{c}, T^{c}\right\}+\frac{3}{5} d^{a b c} d^{c g h}\left\{T^{g}, T^{h}\right\} \\
& +\left(\left\{T^{a}, T^{b}\right\}-\frac{1}{8} \delta^{a b}\left\{T^{c}, T^{c}\right\}-\frac{3}{5} d^{a b c} d^{c g h}\left\{T^{g}, T^{h}\right\}\right), \\
\left\{T^{a}, T^{a}\right\} & =\frac{1}{6}\left(N_{c}+6\right) \mathbb{1}+\left\{J^{k}, J^{k}\right\}, \\
d^{a b c}\left\{T^{a}, T^{b}\right\} & =-\frac{1}{3}\left(N_{c}+3\right) T^{c}+2\left\{J^{i}, G^{i c}\right\} .
\end{aligned}
$$

and

$$
\begin{aligned}
\left\{T^{a}, G^{i b}\right\} & =\frac{1}{2}\left(\left\{T^{a}, G^{i b}\right\}+\left\{T^{b}, G^{i a}\right\}\right)+\frac{1}{2}\left(\left\{T^{a}, G^{i b}\right\}-\left\{T^{b}, G^{i a}\right\}\right), \\
\left\{T^{a}, G^{i b}\right\}+\left\{T^{b}, G^{i a}\right\} & =\frac{1}{4} \delta^{a b}\left\{T^{c}, G^{i c}\right\}+\frac{6}{5} d^{a b c} d^{c g h}\left\{T^{g}, G^{i h}\right\} \\
& +\left(\left\{T^{a}, G^{i b}\right\}+\left\{T^{b}, G^{i a}\right\}-\frac{1}{4} \delta^{a b}\left\{T^{c}, G^{i c}\right\}-\frac{6}{5} d^{a b c} d^{c g h}\left\{T^{g}, G^{i h}\right\}\right), \\
\left\{T^{a}, G^{i b}\right\}-\left\{T^{b}, G^{i a}\right\} & =\frac{2}{3} f^{a b c} f^{c g h}\left\{T^{g}, G^{i h}\right\} \\
& +\left(\left\{T^{a}, G^{i b}\right\}-\left\{T^{b}, G^{i a}\right\}-\frac{2}{3} f^{a b c} f^{c g h}\left\{T^{g}, G^{i h}\right\}\right), \\
\left\{T^{a}, G^{i a}\right\} & =\frac{2}{3}\left(N_{c}+3\right) J^{i}, \\
d^{a b c}\left\{T^{a}, G^{i b}\right\} & =\frac{1}{3}\left(N_{c}+3\right) G^{i c}+\frac{1}{6}\left\{J^{i}, T^{c}\right\}, \\
f^{a b c}\left\{T^{a}, G^{i b}\right\} & =\epsilon^{i j k}\left\{J^{j}, G^{k c}\right\} .
\end{aligned}
$$

\footnotetext{
${ }^{3}$ Contractions with $\delta$ or $\epsilon$ in (G.11) are not in contradiction with the operator reduction rule since these contractions are not applied to the $G$-operator.
} 
The last symmetric product $\left\{G^{i a}, G^{j b}\right\}$ contains three different spin-combination:

$$
\begin{aligned}
\left\{G^{i a}, G^{j b}\right\} & =\frac{1}{3} \delta^{i j}\left\{G^{k a}, G^{k b}\right\}+\frac{1}{2}\left(\left\{G^{i a}, G^{j b}\right\}-\left\{G^{j a}, G^{i b}\right\}\right) \\
& +\underbrace{\frac{1}{2}\left(\left\{G^{i a}, G^{j b}\right\}+\left\{G^{j a}, G^{i b}\right\}-\frac{2}{3} \delta^{i j}\left\{G^{k a}, G^{k b}\right\}\right)}_{\equiv\left\{G^{i a}, G^{j b}\right\}^{(2)}},
\end{aligned}
$$

where $\left\{G^{i a}, G^{j b}\right\}^{(2)}$ abreviates the spin-2 component. We discuss the flavour decomposition of these three pieces separately. The spin- 0 part:

$$
\begin{aligned}
\left\{G^{k a}, G^{k b}\right\} & =\frac{1}{8} \delta^{a b}\left\{G^{k c}, G^{k c}\right\}+\frac{3}{5} d^{a b c} d^{c g h}\left\{G^{k g}, G^{k h}\right\} \\
& +\underbrace{\left(\left\{G^{k a}, G^{k b)}\right\}-\frac{1}{8} \delta^{a b}\left\{G^{k c}, G^{k c}\right\}-\frac{3}{5} d^{a b c} d^{c g h}\left\{G^{k g}, G^{k h}\right\}\right)}_{\equiv\left\{G^{k a}, G^{k b}\right\}}, \\
\left\{G^{k a}, G^{k a}\right\} & =\frac{3}{8}\left(N_{c}+6\right) \mathbb{1}-\frac{5}{12}\left\{J^{k}, J^{k}\right\}, \\
d^{a b c}\left\{G^{k a}, G^{k b}\right\} & =\frac{3}{4}\left(N_{c}+3\right) T^{c}-\frac{7}{6}\left\{J^{k}, G^{k c}\right\}, \\
\left\{G^{k a}, G^{k b}\right\}^{(0,27)} & =\frac{1}{4}\left(\left\{T^{a}, T^{b}\right\}-\frac{1}{8} \delta^{a b}\left\{T^{c}, T^{c}\right\}-\frac{3}{5} d^{a b c} d^{c g h}\left\{T^{g}, T^{h}\right\}\right) .
\end{aligned}
$$

The spin-1 part:

$$
\begin{aligned}
& \left\{G^{i a}, G^{j b}\right\}-\left\{G^{j a}, G^{i b}\right\}=\frac{1}{3} f^{a b c} f^{c g h}\left(\left\{G^{i g}, G^{j h}\right\}-\left\{G^{j g}, G^{i h}\right\}\right) \\
& +\left(\left\{G^{i a}, G^{j b}\right\}-\left\{G^{j a}, G^{i b}\right\}-\frac{1}{3} f^{a b c} f^{c g h}\left(\left\{G^{i g}, G^{j h}\right\}-\left\{G^{j g}, G^{i h}\right\}\right)\right) \\
& =\frac{1}{2} \epsilon^{i j k} \epsilon^{k l m}\left(\frac{1}{3} f^{a b c} f^{c g h}\left\{G^{l g}, G^{m h}\right\}+\left\{G^{l a}, G^{m b}\right\}-\frac{1}{3} f^{a b c} f^{c g h}\left\{G^{l g}, G^{m h}\right\}\right), \\
& \epsilon^{k l m} f^{c g h}\left\{G^{l g}, G^{m h}\right\}=-\left(N_{c}+3\right) G^{k c}+\frac{1}{2}\left\{J^{k}, T^{c}\right\}, \\
& 2 \epsilon^{k l m}\left(\left\{G^{l a}, G^{m b}\right\}-\frac{1}{3} f^{a b c} f^{c g h}\left\{G^{l g}, G^{m h}\right\}\right)=\chi_{-}^{k, a b}-\frac{1}{3} f^{a b c} f^{c g h} \chi_{-}^{k, g h},
\end{aligned}
$$

with

$$
\chi_{-}^{k, a b}=\left(f^{a c g} d^{b c h}-f^{b c g} d^{a c h}\right)\left\{T^{g}, G^{k h}\right\} .
$$

The spin-2 part:

$$
\begin{aligned}
\left\{G^{i a}, G^{j b}\right\}^{(2)} & =\frac{1}{8} \delta^{a b}\left\{G^{i c}, G^{j c}\right\}^{(2)}+\frac{3}{5} d^{a b c} d^{c g h}\left\{G^{i g}, G^{j h}\right\}^{(2)} \\
+ & \left(\left\{G^{i a}, G^{j b}\right\}^{(2)}-\frac{1}{8} \delta^{a b}\left\{G^{i c}, G^{j c}\right\}^{(2)}-\frac{3}{5} d^{a b c} d^{c g h}\left\{G^{i g}, G^{j h}\right\}^{(2)}\right) . \\
\left\{G^{i c}, G^{j c}\right\}^{(2)} & =\frac{1}{3}\left(\left\{J^{i}, J^{j}\right\}-\frac{1}{3} \delta^{i j}\left\{J^{k}, J^{k}\right\}\right), \\
d^{c g h}\left\{G^{i g}, G^{j h}\right\}^{(2)} & =\frac{1}{3}\left(\left\{J^{i}, G^{j c}\right\}+\left\{J^{j}, G^{i c}\right\}-\frac{2}{3} \delta^{i j}\left\{J^{k}, G^{k c}\right\}\right) .
\end{aligned}
$$


Given a product of two one-body operators, where each of them transforms as an $S U(3)$ flavour octet, different flavour multiplets in such a product, denoted by $\chi^{a b}$ in the following, are obtained by contracting the indices with $\delta^{a b}, d^{a b c}$ and $f^{a b c}$ of $S U(3)$. As it was demonstrated above, such contractions can be rewritten with the help of other operators and are redundant. This redundancy is the first part of the operator reduction rule. Whether contractions of two open flavour indices in the operator product with two different $d$ - and/or $f$-symbols, like in

$$
d^{a c g} d^{b c h} \chi^{g h}, \quad f^{a c g} d^{b c h} \chi^{g h}, \quad f^{a c g} f^{b c h} \chi^{g h},
$$

are redundant or not, is not obvious. In order to rewrite these contractions, following properties of $d$ - and $f$-symbols are required:

$$
\begin{aligned}
& d^{a a b}=0, \\
& f^{a b c} f^{a d e} d^{b d f}=\frac{3}{2} d^{c e f}, \\
& d^{a b c} d^{a b d}=\frac{5}{3} \delta^{c d}, \\
& f^{a b c} f^{a b d}=3 \delta^{c d}, \\
& d^{a b c} d^{a d e} f^{b d f}=\frac{5}{6} f^{c e f}, \\
& d^{a b c} d^{a d e} d^{b d f}=-\frac{1}{2} d^{c e f}, \\
& f^{a b c} f^{a d e} f^{b d f}=\frac{3}{2} f^{c e f} .
\end{aligned}
$$

We study the contraction in (G.21) for the symmetric and antisymmetric parts of $\chi^{a b}$ separately. Using the decomposition in (B.20), contractions in (G.21) for the $\overline{\mathbf{1 0}}+\mathbf{1 0}$ and $\mathbf{2 7}$-components in $\chi^{a b}$ given in [22] and the relations in (G.22), one obtains upon a short calculation:

$$
\begin{aligned}
d^{a c g} d^{b c h} \chi_{+}^{g h} & =\frac{5}{24} \delta^{a b} \chi_{1}-\frac{3}{10} d^{a b c} \chi_{8_{S}}^{c}+\frac{1}{3} \chi_{27}^{a b} \\
& =\frac{1}{6} \delta^{a b} \chi_{1}-\frac{1}{2} d^{a b c} \chi_{8_{S}}^{c}+\frac{1}{3} \chi_{+}^{a b}, \\
d^{a c g} d^{b c h} \chi_{-}^{g h} & =\frac{5}{18} f^{a b c} \chi_{8_{A}}^{c}-\frac{2}{3} \chi_{10+10}^{a b} \\
& =\frac{1}{2} f^{a b c} \chi_{8_{A}}^{c}-\frac{2}{3} \chi_{-}^{a b}, \\
f^{a c g} f^{b c h} \chi_{+}^{g h} & =\frac{3}{8} \delta^{a b} \chi_{1}+\frac{9}{10} d^{a b c} \chi_{8_{S}}^{c}-\chi_{27}^{a b} \\
& =\frac{1}{2} \delta^{a b} \chi_{1}+\frac{3}{2} d^{a b c} \chi_{8_{S}}^{c}-\chi_{+}^{a b}, \\
f^{a c g} f^{b c h} \chi_{-}^{g h} & =\frac{1}{2} f^{a b c} \chi_{8_{A}}^{c}, \\
f^{a c g} d^{b c h} \chi_{+}^{g h} & =\frac{1}{2} f^{a b c} \chi_{8_{S}}^{c}, \\
f^{a c g} d^{b c h} \chi_{-}^{g h} & =\frac{5}{6} d^{a b c} \chi_{8_{A}}^{c}+\chi_{i(10-\overline{10})}^{a b} .
\end{aligned}
$$

The above results can also be restated as

$$
\begin{aligned}
d^{a c g} d^{b c h}+d^{a c h} d^{b c g} & =\frac{1}{3}\left(\delta^{a b} \delta^{g h}+\delta^{a g} \delta^{b h}+\delta^{a h} \delta^{b g}\right)-d^{a b c} d^{c g h}, \\
d^{a c g} d^{b c h}-d^{a c h} d^{b c g} & =f^{a b c} f^{c g h}-\frac{2}{3}\left(\delta^{a g} \delta^{b h}-\delta^{a h} \delta^{b g}\right), \\
f^{a c g} f^{b c h}+f^{a c h} f^{b c g} & =\delta^{a b} \delta^{g h}-\delta^{a g} \delta^{b h}-\delta^{a h} \delta^{b g}+3 d^{a b c} d^{c g h},
\end{aligned}
$$




$$
\begin{aligned}
& f^{a c g} f^{b c h}-f^{a c h} f^{b c g}=f^{a b c} f^{c g h}, \\
& f^{a c g} d^{b c h}+f^{a c h} d^{b c g}=f^{a b c} d^{c g h} .
\end{aligned}
$$

Equations in (G.24) are, of course, the well known identities of $d$ and $f$ symbols. The last two, for example, being the Jacobi-identities. Relation of such type can be deduced from the commutation properties of the generators in (B.3) only (see e.g. [66] and the references to the older literature therein). Many of them are compiled in CORE [13]. It is interesting to note, that they can be deduced from the study of the multiplet decomposition of an arbitrary tensor $\chi^{a b}$ and the different contractions with $d$ and $f$ symbols on it.

The relations in (G.24) have an important consequence concerning the relevance of the contractions in (G.21) for the $1 / N_{c^{-}}$expansion in terms of effective quark operators, as discussed in Section 2.3.1 in detail. Symmetric and antisymmetric combinations in (G.24), where the two open flavour indices $a$ and $b$ are distributed among two different $d$ and/or $f$ symbols, are rewritten in terms of tensors where $a$ and $b$ are put on the same $d$ or $f$ symbols or on $\delta$. Such contractions can be eliminated in favour of other operators (first part of the operator reduction rule). However, there is no further identity for the antisymmetric combination

$$
f^{a c g} d^{b c h}-f^{a c h} d^{b c g} .
$$

Contractions of arbitrary products of two one-body operators with two open flavour indices with the above antisymmetric combination cannot be eliminated on the basis of other operators and must to be retained in the course of specifying a complete set of effective quark operators. This exception builds the second part of the operator reduction rule. 


\section{Appendix $\mathrm{H}$.}

\section{Spinors}

In this chapter the matrix elements of

$$
\Gamma \in 1, \gamma_{5}, \gamma^{\mu}, \gamma_{5} \gamma^{\mu}, \sigma^{\mu \nu}
$$

and their momentum dependence are studied for spin- $1 / 2$ and spin- $3 / 2$ spinors. In the Diracrepresentation of the $\gamma$-matrices, the elements of $\Gamma$ read:

$$
\gamma^{0}=\left(\begin{array}{cc}
\mathbb{1} & 0 \\
0 & -\mathbb{1}
\end{array}\right), \quad \gamma^{i}=\left(\begin{array}{cc}
0 & \sigma^{i} \\
-\sigma^{i} & 0
\end{array}\right), \quad \gamma_{5}=\gamma^{5}=i \gamma^{0} \gamma^{1} \gamma^{2} \gamma^{3}=\left(\begin{array}{ll}
0 & \mathbb{1} \\
\mathbb{1} & 0
\end{array}\right)
$$

and

$$
\begin{array}{cc}
\gamma_{5} \gamma^{0}=\left(\begin{array}{cc}
0 & -\mathbb{1} \\
\mathbb{1} & 0
\end{array}\right), & \gamma_{5} \gamma^{i}=\left(\begin{array}{cc}
-\sigma^{i} & 0 \\
0 & \sigma^{i}
\end{array}\right), \\
\sigma^{o j}=i\left(\begin{array}{cc}
0 & \sigma^{j} \\
\sigma^{j} & 0
\end{array}\right), & \sigma^{i j}=\varepsilon_{i j k}\left(\begin{array}{cc}
\sigma^{k} & 0 \\
0 & \sigma^{k}
\end{array}\right),
\end{array}
$$

where $\mathbb{1}$ is a $2 \times 2$-identity matrix. To settle further the notation and the convention used in this work, the construction of the spin-3/2 spinors is discussed first.

Spin-1/2 spinors are given by

$$
u(p, s)=N_{p}\left(\begin{array}{c}
\mathbb{1}_{(2 \times 2)} \\
\frac{\vec{\sigma} \cdot \vec{p}}{E_{p}+M}
\end{array}\right) \chi_{s}^{(1 / 2)}, \quad N_{p}=\sqrt{\frac{E_{p}+M}{2 M}}, \chi_{1}^{(1 / 2)}=\left(\begin{array}{l}
1 \\
0
\end{array}\right), \chi_{2}^{(1 / 2)}=\left(\begin{array}{l}
0 \\
1
\end{array}\right),
$$

with

$$
E_{p}=p^{0}=\sqrt{M^{2}+\vec{p}^{2}}, \quad \text { and } \quad M^{2}=p^{2} .
$$

Spin-3/2 spinors in the Rarita-Schwinger formalism are most economicaly obtained via the coupling of spin-1 polarisation vectors and spin-1/2 spinors $^{1}$ :

$$
u^{\mu}(p, s)=\sum_{\lambda, s^{\prime}} C\left(1 \lambda \frac{1}{2} s^{\prime} \mid \frac{3}{2} s\right) \varepsilon_{\lambda}^{\mu}(p) u_{s^{\prime}}(p), \quad s^{\prime}= \pm \frac{1}{2}, \quad \lambda=0, \pm 1 .
$$

${ }^{1}$ Phase convention for the Clebsch-Gordan coefficients, that is very common in the physical literature, is used here. The eigenvalues of the raising and lowering operators are real and positive

$$
J_{ \pm}|j, m\rangle=\sqrt{j(j+1)-(m(m \pm 1)}|j, \pm m\rangle
$$

and the coefficient for the "maximal coupling" of two states $\left|j_{1}, m_{1}\right\rangle$ and $\left|j_{2}, m_{2}\right\rangle$ to $\left|j_{1}+j_{2}, m_{1}+m_{2}\right\rangle$ ist 1:

$$
\left|j_{1}+j_{2}, m_{1}+m_{2}\right\rangle=\left|j_{1}, m_{1}\right\rangle \otimes\left|j_{2}, m_{2}\right\rangle
$$


To specify the spin- 1 polarisation vectors $\varepsilon^{\mu}(p, \lambda)$, we pick out a frame where $\vec{p}$ is along the $z$-axis, $p^{\mu}=(0,0,0,|\vec{p}|)$. In this special frame the spacelike polarisation vectors take a simple form:

$$
\varepsilon^{\mu}(p, 1)=\left(\begin{array}{l}
0 \\
1 \\
0 \\
0
\end{array}\right), \quad \varepsilon^{\mu}(p, 2)=\left(\begin{array}{l}
0 \\
0 \\
1 \\
0
\end{array}\right), \quad \varepsilon^{\mu}(p, 3)=\left(\begin{array}{c}
\frac{|\vec{p}|}{M} \\
0 \\
0 \\
\frac{p^{0}}{M}
\end{array}\right) .
$$

The basis in the Minkowski space is completed by the fourth timelike vector

$$
\varepsilon^{\mu}(p, 0)=\frac{p^{\mu}}{M}
$$

The polarisation vectors are subject to the constraints

$$
p_{\mu} \varepsilon^{\mu}(p, 0)=M, \quad p_{\mu} \varepsilon^{\mu}(p, \lambda)=0, \quad \lambda=1,2,3 .
$$

Furthermore, the orthonormality and completeness relations are

$$
\varepsilon_{\mu}^{*}\left(p, \lambda^{\prime}\right) \varepsilon^{\mu}(p, \lambda)=-\delta_{\lambda^{\prime} \lambda}, \quad \sum_{\lambda} \varepsilon^{\mu}(p, \lambda) \varepsilon^{\nu}(p, \lambda)=-g^{\mu \nu}+p^{\mu} p^{\nu} / m^{2} .
$$

In cartesian representation the eigenvectors of the third spin matrix for a vector field are given by the spherical basis vectors $\varepsilon_{\lambda}^{\mu}$ with $\lambda= \pm 1,0$ (see e.g. [76]):

$$
\varepsilon_{ \pm}^{\mu}(p)=\frac{1}{\sqrt{2}}\left(\mp \varepsilon^{\mu}(p, 1)-i \varepsilon^{\mu}(p, 2)\right), \quad \varepsilon_{0}^{\mu}(p)=\varepsilon^{\mu}(p, 3) .
$$

The explicit form of the vector-spinors is ${ }^{2}$ :

$$
\begin{aligned}
u^{\mu}(p, 1) & =\varepsilon_{+}^{\mu}(p) u(p, 1) \\
& =\frac{N_{p}}{\sqrt{2}}\left(\left(\begin{array}{c}
0 \\
0
\end{array}\right),-\left(\begin{array}{c}
\chi_{1} \\
\frac{\vec{\sigma} \cdot \vec{p}}{E_{p}+M} \chi_{1}
\end{array}\right),-i\left(\begin{array}{c}
\chi_{1} \\
\frac{\vec{\sigma} \cdot \vec{p}}{E_{p}+M} \chi_{1}
\end{array}\right),\left(\begin{array}{c}
0 \\
0
\end{array}\right)\right), \\
u^{\mu}(p, 2) & =\frac{1}{\sqrt{3}} \varepsilon_{+}^{\mu}(p) u(p, 2)+\sqrt{\frac{2}{3}} \varepsilon_{0}^{\mu}(p) u(p, 1) \\
& =\frac{N_{p}}{\sqrt{6}}\left(2 \frac{|\vec{p}|}{M}\left(\begin{array}{c}
\chi_{1} \\
\frac{\vec{\sigma} \cdot \vec{p}}{E_{p}+M} \chi_{1}
\end{array}\right),-\left(\begin{array}{c}
\chi_{2} \\
\frac{\vec{\sigma} \cdot \vec{p}}{E_{p}+M} \chi_{2}
\end{array}\right),-i\left(\begin{array}{c}
\chi_{2} \\
\frac{\vec{\sigma} \cdot \vec{p}}{E_{p}+M} \chi_{2}
\end{array}\right), 2 \frac{E_{p}}{M}\left(\begin{array}{c}
\chi_{1} \\
\frac{\vec{\sigma} \cdot \vec{p}}{E_{p}+M} \chi_{1}
\end{array}\right)\right), \\
u^{\mu}(p, 3) & =\frac{1}{\sqrt{3}} \varepsilon_{-}^{\mu}(p) u(p, 1)+\sqrt{\frac{2}{3}} \varepsilon_{0}^{\mu}(p) u(p, 2) \\
& =\frac{N_{p}}{\sqrt{6}}\left(2 \frac{|\vec{p}|}{M}\left(\begin{array}{c}
\chi_{2} \\
\frac{\vec{\sigma} \cdot \vec{p}}{E_{p}+M} \chi_{2}
\end{array}\right),\left(\begin{array}{c}
\chi_{1} \\
\frac{\vec{\sigma} \cdot \vec{p}}{E_{p}+M} \chi_{1}
\end{array}\right),-i\left(\begin{array}{c}
\chi_{1} \\
\frac{\vec{\sigma} \cdot \vec{p}}{E_{p}+M} \chi_{1}
\end{array}\right), 2 \frac{E_{p}}{M}\left(\begin{array}{c}
\chi_{2} \\
\frac{\vec{\sigma} \cdot \vec{p}}{E_{p}+M} \chi_{2}
\end{array}\right)\right), \\
u^{\mu}(p, 4) & =\frac{N_{-}^{\mu}(p) u(p, 2)}{\sqrt{2}}\left(\left(\begin{array}{c}
0 \\
0
\end{array}\right),\left(\begin{array}{c}
\chi_{2} \\
\frac{\vec{\sigma} \cdot \vec{p}}{E_{p}+M} \chi_{2}
\end{array}\right),-i\left(\begin{array}{c}
\chi_{2} \\
\frac{\vec{\sigma} \cdot \vec{p}}{E_{p}+M} \chi_{2}
\end{array}\right),\left(\begin{array}{c}
0 \\
0
\end{array}\right)\right) .
\end{aligned}
$$

\footnotetext{
${ }^{2}$ To simplify the notation, in the next few lines $\chi_{1}$ and $\chi_{2}$ are used for $\chi_{1}^{(1 / 2)}$ and $\chi_{2}^{(1 / 2)}$, respectively.
} 
Similar to (H.4), $u^{\mu}(p, s)$ can be written as

$$
u^{\mu}(p, s)=N_{p} \tilde{S}^{\mu \dagger}(p) \chi_{s}^{(3 / 2)}, \quad s=1, \ldots 4,
$$

with

$$
\chi_{1}^{(3 / 2)}=\left(\begin{array}{l}
1 \\
0 \\
0 \\
0
\end{array}\right), \quad \chi_{2}^{(3 / 2)}=\left(\begin{array}{l}
0 \\
1 \\
0 \\
0
\end{array}\right), \quad \chi_{3}^{(3 / 2)}=\left(\begin{array}{l}
0 \\
0 \\
1 \\
0
\end{array}\right), \quad \chi_{4}^{(3 / 2)}=\left(\begin{array}{l}
0 \\
0 \\
0 \\
1
\end{array}\right)
$$

and

$$
\begin{aligned}
& \tilde{S}^{0 \dagger}(p)=\frac{|\vec{p}|}{M}\left(\begin{array}{cccc}
0 & \sqrt{\frac{2}{3}} \chi_{1} & \sqrt{\frac{2}{3}} \chi_{2} & 0 \\
0 & \sqrt{\frac{2}{3}} \frac{\vec{\sigma} \cdot \vec{p}}{E_{p}+M} \chi_{1} & \sqrt{\frac{2}{3}} \frac{\vec{\sigma} \cdot \vec{p}}{E_{p}+M} \chi_{2} & 0
\end{array}\right), \\
& \tilde{S}^{1 \dagger}(p)=\left(\begin{array}{cccc}
-\frac{1}{\sqrt{2}} \chi_{1} & -\frac{1}{\sqrt{6}} \chi_{2} & \frac{1}{\sqrt{6}} \chi_{1} & \frac{1}{\sqrt{2}} \chi_{2} \\
-\frac{1}{\sqrt{2}} \frac{\vec{\sigma} \cdot \vec{p}}{E_{p}+M} \chi_{1} & -\frac{1}{\sqrt{6}} \frac{\vec{\sigma} \cdot \vec{p}}{E_{p}+M} \chi_{2} & \frac{1}{\sqrt{6}} \frac{\vec{\sigma} \cdot \vec{p}}{E_{p}+M} \chi_{1} & \frac{1}{\sqrt{2}} \frac{\vec{\sigma} \cdot \vec{p}}{E_{p}+M} \chi_{2}
\end{array}\right), \\
& \tilde{S}^{2 \dagger}(p)=\left(\begin{array}{cccc}
-\frac{i}{\sqrt{2}} \chi_{1} & -\frac{i}{\sqrt{6}} \chi_{2} & \frac{i}{\sqrt{6}} \chi_{1} & \frac{i}{\sqrt{2}} \chi_{2} \\
-\frac{i}{\sqrt{2}} \frac{\vec{\sigma} \cdot \vec{p}}{E_{p}+M} \chi_{1} & -\frac{i}{\sqrt{6}} \frac{\vec{\sigma} \cdot \vec{p}}{E_{p}+M} \chi_{2} & -\frac{i}{\sqrt{6}} \frac{\vec{\sigma} \cdot \vec{p}}{E_{p}+M} \chi_{1} & -\frac{i}{\sqrt{2}} \frac{\vec{\sigma} \cdot \vec{p}}{E_{p}+M} \chi_{2}
\end{array}\right), \\
& \tilde{S}^{3 \dagger}(p)=\frac{E_{p}}{M}\left(\begin{array}{cccc}
0 & \sqrt{\frac{2}{3}} \chi_{1} & \sqrt{\frac{2}{3}} \chi_{2} & 0 \\
0 & \sqrt{\frac{2}{3}} \frac{\vec{\sigma} \cdot \vec{p}}{E_{p}+M} \chi_{1} & \sqrt{\frac{2}{3}} \frac{\vec{\sigma} \cdot \vec{p}}{E_{p}+M} \chi_{2} & 0
\end{array}\right) .
\end{aligned}
$$

In the static limit the matrices $\tilde{S}$ reduce to ${ }^{3,4}$

$$
\tilde{S}^{\mu \dagger}=\left(0, \tilde{S}^{i \dagger}\right) .
$$

The $4 \times 4$ matrices $\tilde{S}^{\mu \dagger}(p)$ can be given in a more compact form:

$$
\tilde{S}^{\mu \dagger}(p)=\left(\begin{array}{c}
S^{\mu \dagger}(p) \\
\frac{\vec{\sigma} \cdot \vec{p}}{E_{p}+M} S^{\mu \dagger}(p)
\end{array}\right),
$$

with

$$
\begin{array}{rlrl}
S^{0 \dagger}(p) & =\frac{|\vec{p}|}{M}\left(\begin{array}{cccc}
0 & \sqrt{\frac{2}{3}} & 0 & 0 \\
0 & 0 & \sqrt{\frac{2}{3}} & 0
\end{array}\right), & S^{1 \dagger}(p)=\left(\begin{array}{cccc}
-\frac{1}{\sqrt{2}} & 0 & \frac{1}{\sqrt{6}} & 0 \\
0 & -\frac{1}{\sqrt{6}} & 0 & \frac{1}{\sqrt{2}}
\end{array}\right), \\
S^{2 \dagger}(p)=\left(\begin{array}{cccc}
-\frac{i}{\sqrt{2}} & 0 & \frac{-i}{\sqrt{6}} & 0 \\
0 & -\frac{-i}{\sqrt{6}} & 0 & \frac{-i}{\sqrt{2}}
\end{array}\right), & S^{3 \dagger}(p)=\frac{E_{p}}{M}\left(\begin{array}{cccc}
0 & \sqrt{\frac{2}{3}} & 0 & 0 \\
0 & 0 & \sqrt{\frac{2}{3}} & 0
\end{array}\right) .
\end{array}
$$

Using the matrices above, the spin-3/2 spinors can be given in a form similar to (H.4):

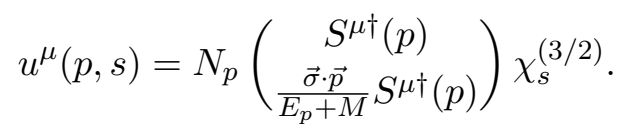

\footnotetext{
${ }^{3}$ In the following, by not writing out the explicit dependence of objects under consideration on the momentum $p$, the static case with $\vec{p}=0$ is meant.

${ }^{4}$ The vanishing of the 0 -th field component is common to all fields with spin $s \geqq 1$.
} 
Important properties of $\tilde{S}$ - and $S$ - matrices are obtained by considering the projection operator over the positive energy states:

$$
\Lambda_{+}^{\mu \nu}(p)=\sum_{s} u^{\mu}(p, s) \bar{u}^{\nu}(p, s)=-\frac{\not p+M}{2 M}\left(g^{\mu \nu}-\frac{1}{3} \gamma^{\mu} \gamma^{\nu}-\frac{2}{3} \frac{p^{\mu} p^{\nu}}{M^{2}}+\frac{p^{\mu} \gamma^{\nu}-p^{\nu} \gamma^{\mu}}{3 M}\right) .
$$

In the static limit only the space components survive:

$$
\Lambda_{+}^{00}=\Lambda_{+}^{0 i}=\Lambda_{+}^{i 0}=0, \quad \Lambda_{+}^{i j}=\frac{1}{2}\left(\gamma^{0}+1\right)\left(\delta^{i j}+\frac{1}{3} \gamma^{i} \gamma^{j}\right), \quad i, j=1,2,3 .
$$

Rewriting this spin-sum with the help of (H.21) leads to

$$
\begin{aligned}
\Lambda_{+}^{\mu \nu}(p) & =N_{p}^{2} \sum_{s} \tilde{S}^{\mu \dagger}(p) \chi_{s} \chi_{s}^{\dagger} \tilde{S}^{\nu}(p)=N_{p}^{2} \tilde{S}^{\mu \dagger}(p) \tilde{S}^{\nu}(p) \\
& =N_{p}^{2}\left(\begin{array}{cc}
S^{\mu \dagger}(p) S^{\nu}(p) & S^{\mu \dagger}(p) S^{\nu}(p) \frac{\vec{\sigma} \cdot \vec{p}}{E_{p}+M} \\
\frac{\vec{\sigma} \cdot \vec{p}}{E_{p}+M} S^{\mu \dagger}(p) S^{\nu}(p) & \frac{\vec{\sigma} \cdot \vec{p}}{E_{p}+M} S^{\mu \dagger}(p) S^{\nu}(p) \frac{\vec{\sigma} \cdot \vec{p}}{E_{p}+M}
\end{array}\right) .
\end{aligned}
$$

Comparing of (H.22) and (H.24) leads directly to

$$
\begin{aligned}
\tilde{S}^{\mu \dagger}(p) \tilde{S}^{\nu}(p) & =-\frac{\not p+M}{E_{p}+M}\left(g^{\mu \nu}-\frac{1}{3} \gamma^{\mu} \gamma^{\nu}-\frac{2}{3} \frac{p^{\mu} p^{\nu}}{M^{2}}+\frac{p^{\mu} \gamma^{\nu}-p^{\nu} \gamma^{\mu}}{3 M}\right), \\
S^{0 \dagger}(p) S^{0}(p) & =\frac{2}{3} \frac{\vec{p}^{2}}{m^{2}} \mathbb{1}_{2 \times 2},
\end{aligned}
$$

and for the non-vanishing space-components at $\vec{p}=0$ to

$$
\tilde{S}^{i \dagger} \tilde{S}^{j}=\frac{1}{2}\left(\gamma^{0}+1\right)\left(\delta^{i j}+\frac{1}{3} \gamma^{i} \gamma^{j}\right), \quad S^{i \dagger} S^{j}=\delta^{i j}-\frac{1}{3} \sigma^{i} \sigma^{j} .
$$

Using the explicit form of spinors in (H.4) and in (H.21), the matrix elements of $\Gamma$ can be written down with the help of $\chi_{s}^{(1 / 2)}$ and $\chi_{s}^{(3 / 2)}$ :

$$
\begin{aligned}
\bar{u}\left(p^{\prime}, s^{\prime}\right) \Gamma u(p, s) & =\chi_{s^{\prime}}^{\dagger(1 / 2)} \tilde{\Gamma} \chi_{s}^{(1 / 2)}, \\
\bar{u}^{\mu}\left(p^{\prime}, s^{\prime}\right) \Gamma u^{\nu}(p, s) & =\chi_{s^{\prime}}^{\dagger(3 / 2)}\left(S^{\mu}\left(p^{\prime}\right) \tilde{\Gamma} S^{\nu \dagger}(p)\right) \chi_{s}^{(3 / 2)}, \\
\bar{u}^{\mu}\left(p^{\prime}, s^{\prime}\right) \Gamma u(p, s) & =\chi_{s^{\prime}}^{\dagger(3 / 2)}\left(S^{\mu}\left(p^{\prime}\right) \tilde{\Gamma}\right) \chi_{s}^{(1 / 2)} .
\end{aligned}
$$

The structures $\tilde{\Gamma}$ for the specific form of the elements of $\Gamma$ in the Dirac-representation and their non-relativistic expansions are collected in Table H.1. Products $S^{i} S^{j \dagger}$ and $S^{i} \sigma^{j}$, which occur during the evaluation of (H.27), can be decomposed further into invariant subspaces. This decomposition and some other properties of $S$ - and $\sigma$-matrices, that are frequently used in this work, are compiled in Chapter F.

The normalisation of spinors follows directly from (H.27) with $\Gamma=\mathbb{1}$ :

$$
\bar{u}\left(p, s^{\prime}\right) u(p, s)=\delta_{s^{\prime} s}, \quad \bar{u}_{\mu}\left(p, s^{\prime}\right) u^{\mu}(p, s)=-\delta_{s^{\prime} s},
$$

where for the spin- $3 / 2$ spinors the relation

$$
S_{\mu}(p) S^{\mu \dagger}(p)=-\mathbb{1}_{4 \times 4}
$$

was used ${ }^{5}$. This relation can be proven by using the explicit form of the matrices in (H.20).

\footnotetext{
${ }^{5}$ The normalisation of the spin-3/2 spinors can alternatively be checked with the help of the definition in (H.8), the completeness relation in and the orthogonality properties of the Clebsh-Gordan-coefficients.
} 


\begin{tabular}{|c|c|c|}
\hline$\Gamma$ & $\tilde{\Gamma}$ & $\tilde{\Gamma}$, n.r.e. \\
\hline $\mathbb{1}$ & $N_{p^{\prime}} N_{p}\left(1-\frac{\vec{\sigma} \cdot \vec{p}^{\prime}}{E_{p^{\prime}}+M} \frac{\vec{\sigma} \cdot \vec{p}}{E_{p}+M}\right)$ & 1 \\
$\gamma_{5}$ & $N_{p^{\prime}} N_{p}\left(\frac{\vec{\sigma} \cdot \vec{p}}{E_{p}+M}-\frac{\vec{\sigma} \cdot \vec{p}^{\prime}}{E_{p^{\prime}}+M}\right)$ & $\frac{1}{2 M} \vec{\sigma} \cdot\left(\vec{p}-\vec{p}^{\prime}\right)$ \\
$\gamma^{0}$ & $N_{p^{\prime}} N_{p}\left(1+\frac{\vec{\sigma} \cdot \vec{p}^{\prime}}{E_{p^{\prime}}+M} \frac{\vec{\sigma} \cdot \vec{p}}{E_{p}+M}\right)$ & 1 \\
$\vec{\gamma}$ & $N_{p^{\prime}} N_{p}\left(\vec{\sigma} \frac{\vec{\sigma} \cdot \vec{p}}{E_{p}+M}+\frac{\vec{\sigma} \cdot \vec{p}^{\prime}}{E_{p^{\prime}}+M} \vec{\sigma}\right)$ & $\frac{1}{2 M}\left(\left(\vec{p}+\vec{p}^{\prime}\right)+i\left(\vec{p}-\vec{p}^{\prime}\right) \times \vec{\sigma}\right)$ \\
$\gamma^{0} \gamma_{5}$ & $N_{p^{\prime}} N_{p}\left(\frac{\vec{\sigma} \cdot \vec{p}^{\prime}}{E_{p^{\prime}}+M}+\frac{\vec{\sigma} \cdot \vec{p}}{E_{p}+M}\right)$ & $\frac{1}{2 M} \vec{\sigma} \cdot\left(\vec{p}+\vec{p}^{\prime}\right)$ \\
$\vec{\gamma} \gamma_{5}$ & $N_{p^{\prime}} N_{p}\left(\vec{\sigma}+\frac{\vec{\sigma} \cdot \vec{p}^{\prime}}{E_{p^{\prime}}+M} \vec{\sigma} \frac{\vec{\sigma} \cdot \vec{p}}{E_{p}+M}\right)$ & $\vec{\sigma}$ \\
$\sigma^{0 j}$ & $N_{p^{\prime}} N_{p} i\left(\sigma^{j} \frac{\vec{\sigma} \cdot \vec{p}}{E_{p}+M}-\frac{\vec{\sigma} \cdot \vec{p}^{\prime}}{E_{p^{\prime}}+M} \sigma^{j}\right)$ & $\frac{i}{2 M}\left(\left(p^{j}-p^{\prime j}\right)+i \epsilon^{j k l}\left(p^{k}+p^{\prime k}\right) \sigma^{l}\right)$ \\
$\sigma^{i j}$ & $N_{p^{\prime}} N_{p} \varepsilon^{i j k}\left(\sigma^{k}-\frac{\vec{\sigma} \cdot \vec{p}^{\prime}}{E_{p}^{\prime}+M} \sigma^{k} \frac{\vec{\sigma} \cdot \vec{p}}{E_{p}+M}\right)$ & $\varepsilon^{i j k} \sigma^{k}$ \\
\hline
\end{tabular}

Table H.1.: $\tilde{\Gamma}$, as definied in (H.27) and the non-relativistic expansion (n.r.e.) of it for $M^{\prime}=M$ up to the order $\mathcal{O}\left(Q^{2} / M^{2}\right)$ with $|\vec{p}|,\left|\vec{p}^{\prime}\right|=\mathcal{O}(Q)$. 


\section{Bibliography}

[1] S. Aoki et al. 2+1 Flavor Lattice QCD toward the Physical Point. Phys. Rev., D79:034503, 2009.

[2] S. Aoki et al. Physical Point Simulation in 2+1 Flavor Lattice QCD. 2009.

[3] T. Appelquist and J. Carazzone. Infrared Singularities and Massive Fields. Phys. Rev., D11:2856, 1975.

[4] C. Aubin et al. Light hadrons with improved staggered quarks: Approaching the continuum limit. Phys. Rev., D70:094505, 2004.

[5] W. A. Bardeen. Anomalous Ward identities in spinor field theories. Phys. Rev., 184:1848-1857, 1969.

[6] T. Becher and H. Leutwyler. Baryon chiral perturbation theory in manifestly lorentz invariant form. Eur. Phys. J. C, 9:643, 1999.

[7] Paulo F. Bedaque and Markus A. Luty. Baryon masses at second order in large- $N$ chiral perturbation theory. Phys. Rev., D54:2317-2327, 1996.

[8] V. Bernard, T. R. Hemmert, and U.-G. Meiner. Infrared regularization with spin-3/2 fields. Phys. Lett. B, 565:137, 2003.

[9] J. Bijnens, G. Ecker, and J. Gasser. Chiral perturbation theory. 1994.

[10] B. Borasoy. Baryon axial currents. Phys. Rev., D59:054021, 1999.

[11] B. Borasoy, B. R. Holstein, R. Lewis, and P. P. A. Ouimet. Long distance regularization in chiral perturbation theory with decuplet fields. Phys. Rev., D66:094020, 2002.

[12] B. Borasoy and Ulf-G. Meissner. Chiral expansion of baryon masses and sigma-terms. Annals Phys., 254:192-232, 1997.

[13] V. I. Borodulin, R. N. Rogalev, and S. R. Slabospitsky. CORE: COmpendium of RElations: Version 2.1. 1995. 
[14] M. Bourquin et al. Measurements of hyperon semileptonic decays at the cern super proton synchrotron. 1 . the sigma- $\rightarrow$ lambda e- anti-neutrino decay mode. Zeit. Phys., C12:307, 1982.

[15] M. N. Butler, M. J. Savage, and R. P. Springer. Strong and elektromagnetic decays of the baryon decuplet. Nuclear Physics B, 399:69-85, 1993.

[16] C. G. Callan, S. Colleman, J. Wess, and B. Zumino. Structure of phenomenological lagrangians. ii. Phys. Rev., 177:2247, 1969.

[17] S. Coleman. The Invariance of the Vacuum is the Invariance of the World. J. Math. Phys., 7:787, 1966.

[18] S. Coleman. Aspects of symmetry. Cambridge University Press, 1988.

[19] S. Colleman, J. Wess, and B. Zumino. Structure of phenomenological lagrangians. i. Phys. Rev., 177:2239, 1969.

[20] R. F. Dashen. Chiral SU(3) x SU(3) as a symmetry of the strong interactions. Phys. Rev., 183:1245-1260, 1969.

[21] R. F. Dashen, E. E. Jenkins, and A. V. Manohar. The 1/n(c) expansion for baryons. Phys. Rev., D49:4713-4738, 1994.

[22] R. F. Dashen, E. E. Jenkins, and A. V. Manohar. Spin flavor structure of large n(c) baryons. Phys. Rev., D51:3697-3727, 1995.

[23] R. F. Dashen and M. Weinstein. Soft pions, chiral symmetry, and phenomenological lagrangians. Phys. Rev., 183:1261-1291, 1969.

[24] P. Dittner. Invariant tensors in su(3). Commun. Math. Phys., 22:238-252, 1971.

[25] J. F. Donoghue and B. R. Holstein. Improved treatment of loop diagrams in su(3) baryon chiral perturbation theory. Phys. Lett. B, 436:331, 1998.

[26] J. F. Donoghue, B. R. Holstein, and B Borasoy. SU(3) baryon chiral perturbation theory and long distance regularization. Phys. Rev., D59:036002, 1999.

[27] S. Durr et al. Ab-Initio Determination of Light Hadron Masses. Science, 322:12241227, 2008.

[28] P. J. Ellis and H.-B. Tang. Pion-nucleon scattering in a new approach to chiral perturbation theory. Phys. Rev. C, 57:3356, 1998. 
[29] S. Eidelman et al. Review of particle physics. Phys. Lett. B, 592:1, 2004.

[30] M. Frink and U.-G. Meissner. Chiral extrapolations of baryon masses for unquenched three-flavor lattice simulations. JHEP, 07:028, 2004.

[31] M. Frink and U.-G. Meissner. On the chiral effective meson-baryon Lagrangian at third order. Eur. Phys. J., A29:255-260, 2006.

[32] T. Fuchs, J. Gegelia, G. Japaridze, and S. Scherer. Renormalization of relativistic baryon chiral perturbation theory and power counting. Phys. Rev. D, 68:056005, 2003.

[33] S. Gasiorowicz. Elementary particle physics. Jon Wiley and Sons, Inc., 1966.

[34] J. Gasser and H. Leutwyler. Quark Masses. Phys. Rept., 87:77-169, 1982.

[35] J. Gasser and H. Leutwyler. Low-Energy Theorems as Precision Tests of QCD. Phys. Lett., B125:325, 1983.

[36] J. Gasser and H. Leutwyler. On the low-energy structure of qcd. Phys. Lett., B125:321, 1983.

[37] J. Gasser and H. Leutwyler. Chiral Perturbation Theory to One Loop. Ann. Phys., 158:142, 1984.

[38] J. Gasser and H. Leutwyler. Chiral Perturbation Theory: Expansions in the Mass of the Strange Quark. Nucl. Phys., B250:465, 1985.

[39] J. Gasser, M. E. Sainio, and A. Svarc. Nucleons with chiral loops. Nucl. Phys. B, 307:779, 1988.

[40] J. Gegelia and G. Japaridze. Matching heavy particle approach to relativistic theory. Phys. Rev., D60:114038, 1999.

[41] J. Gegelia, G. Japaridze, and X. Q. Wang. Is heavy baryon approach necessary? J. Phys., G29:2303-2309, 2003.

[42] M. Gell-Mann and Y. Ne'eman. The Eightfold Way. Benjamin, New York, 1964.

[43] J. L. Gervais and B. Sakita. Large- $n$ qcd baryon dynamics ${ }^{-}$exact results from its relation to the static strong-coupling theory. Phys. Rev. Lett., 52(2):87-89, Jan 1984.

[44] W. Greiner and B Müller. Quantenmechanik: Symmetrien. Verlag Harri Deutsch, 2005. 
[45] R. Haag. Quantum field theories with composite particles and asymptotic conditions. Phys. Rev., 112:669-673, 1958.

[46] H. Hamber and G. Parisi. Numerical Estimates of Hadronic Masses in a Pure SU(3) Gauge Theory. Phys. Rev. Lett., 47:1792, 1981.

[47] C. Itzykson and J.-B. Zuber. Quantum Field Theory. McGRAW-Hill, 1980.

[48] B. Sakita J.-L. Gervais. Large-n baryonic soliton and quarks. Phys. Rev. D, 30(8):1795-1804, 1984.

[49] R. L. Jaffe and A. V. Manohar. The G(1) Problem: Fact and Fantasy on the Spin of the Proton. Nucl. Phys., B337:509-546, 1990.

[50] E. E. Jenkins and R. F. Lebed. Baryon mass splittings in the $1 / \mathrm{n}(\mathrm{c})$ expansion. Phys. Rev., D52:282-294, 1995.

[51] E. E. Jenkins and A. V. Manohar. Baryon chiral perturbation theory using a heavy fermion lagrangian. Phys. Lett. B, 255:558, 1991.

[52] E. E. Jenkins and A. V. Manohar. Chiral corrections to the baryon axial currents. Phys. Lett., B259:353-358, 1991.

[53] E. E. Jenkins and A. V. Manohar. 1/N(c) expansion for exotic baryons. JHEP, 06:039, 2004.

[54] E. E. Jenkins, A. V. Manohar, J. W. Negele, and A. Walker-Loud. A Lattice Test of 1 over Nc Baryon Mass Relations. Phys. Rev., D81:014502, 2010.

[55] S. Kamefuchi, L. O'Raifeartaigh, and A. Salam. Change of variables and equivalence theorems in quantum field theories. Nucl. Phys., 28:529, 1961.

[56] G. Karl and H. J. Lipkin. Flavor symmetry in the large N(c) limit. Phys. Lett., B279:347-351, 1992.

[57] A. Krause. Baryon matrix elements of the vector current in chiral perturbation theory. Helv. Phys. Acta, 63:3-70, 1990.

[58] H. Krebs, E. Epelbaum, and U. G. Meissner. Redundancy of the off-shell parameters in chiral effective field theory with explicit spin-3/2 degrees of freedom. 2009.

[59] T.D. Lee. Particle Physics and Introduction to Field Theory.

[60] B. C. Lehnhart, J. Gegelia, and S. Scherer. Baryon masses and nucleon sigma terms 
in manifestly Lorentz-invariant baryon chiral perturbation theory. J. Phys., G31:89$104,2005$.

[61] H. Leutwyler. Light quark masses. 2009.

[62] H.-W. Lin et al. First results from $2+1$ dynamical quark flavors on an anisotropic lattice: light-hadron spectroscopy and setting the strange-quark mass. Phys. Rev., D79:034502, 2009.

[63] M. A. Luty and J. March-Russell. Baryons from quarks in the $1 / \mathrm{n}$ expansion. Nucl. Phys., B426:71-93, 1994.

[64] M. F. M. Lutz and E. E. Kolomeitsev. Relativistic chiral su(3) symmetry, large $n_{c}$ sum rules and meson-baryon scattering. Nucl. Phys. A, 700:193, 2002.

[65] M. F. M. Lutz and C. L. Korpa. Self consistent propagation of hyperons and antikaons in nuclear matter based on relativistic chiral su(3) dynamics. Nucl. Phys. A, 700:309, 2002.

[66] A. J. MacFarlane, Anthony Sudbery, and P. H. Weisz. On Gell-Mann's gamma matrices, d tensors and f tensors, octets, and parametrizations of $\mathrm{SU}(3)$. Commun. Math. Phys., 11:77-90, 1968.

[67] Y. Makeenko. Large-N gauge theories. 1999.

[68] A. V. Manohar. Large n qcd. hep-ph/9802419, 1998.

[69] L. M. Nath, B. Etemadi, and J. D. Kimel. Uniqueness of the interaction involving spin 3/2 particles. Phys. Rev., D3:2153-2161, 1971.

[70] Y.-S. Oh and W. Weise. Baryon masses in large N(c) chiral perturbation theory. Eur. Phys. J., A4:363-380, 1999.

[71] J. A. Oller, M. Verbeni, and J. Prades. Meson-baryon effective chiral lagrangians to $\mathrm{O}\left(\mathrm{q}^{* *} 3\right)$. JHEP, 09:079, 2006.

[72] J. A. Oller, M. Verbeni, and J. Prades. Meson - baryon effective chiral Lagrangian at $\mathrm{O}\left(\mathrm{q}^{* * 3}\right)$ revisited. 2007.

[73] B. A. Ovrut and H. J. Schnitzer. Decoupling theorems for effective field theories. Phys. Rev., D22:2518, 1980.

[74] V. Pascalutsa. Correspondence of consistent and inconsistent spin-3/2 couplings via the equivalence theorem. Phys. Lett., B503:85-90, 2001. 
[75] M. E. Peskin and D. V. Schroeder. An Introduction to QFT. Westview Press, 1995.

[76] M. E. Rose. Elementary theory of angular momentum. John Wiley Sons Inc, 1957.

[77] L. H. Ryder. Quantum Field Theory. Cambridge University Press, 1985.

[78] S. Scherer. Introduction to chiral perturbation theory. Adv. Nucl. Phys., 27:277, 2003.

[79] A. Semke and M. F. M. Lutz. Baryon self energies in the chiral loop expansion. Nucl. Phys., A778:153-180, 2006.

[80] A. Semke and M. F. M. Lutz. On the possibility of a discontinuous quark-mass dependence of baryon octet and decuplet masses. Nucl. Phys., A789:251-259, 2007.

[81] H. E. Stanley. Spherical model as the limit of infinite spin dimensionality. Phys. Rev., 176:718-722, 1968.

[82] G. 't Hooft. A planar diagram theory for strong interactions. Nucl. Phys., B72:461, 1974.

[83] G. 't Hooft. A Two-Dimensional Model for Mesons. Nucl. Phys., B75:461, 1974.

[84] G. 't Hooft. Symmetry breaking through Bell-Jackiw anomalies. Phys. Rev. Lett., $37: 8-11,1976$.

[85] G. 't Hooft. Large N. 2002.

[86] H.-B. Tang and P. J. Ellis. Redundance of Delta isobar parameters in effective field theories. Phys. Lett., B387:9-13, 1996.

[87] B. C. Tiburzi and A. Walker-Loud. Decuplet baryon masses in partially quenched chiral perturbation theory. Nucl. Phys., A748:513-536, 2005.

[88] A. Walker-Loud et al. Light hadron spectroscopy using domain wall valence quarks on an Asqtad sea. 2008.

[89] S. Weinberg. Nonlinear realizations of chiral symmetry. Phys. Rev., 166:1568, 1968.

[90] S. Weinberg. Phenomenological lagrangians. Physica A, 96:327, 1979.

[91] S. Weinberg. Quantum Theory of Fields, II. Cambridge University Press, 1996. 
[92] J. Wess and B. Zumino. Consequences of anomalous Ward identities. Phys. Lett., B37:95, 1971.

[93] K. G. Wilson. Quantum field theory models in less than four- dimensions. Phys. Rev., D7:2911-2926, 1973.

[94] K. G. Wilson. The renormalization group and critical phenomena. Rev. Mod. Phys., 55(3):583-600, Jul 1983.

[95] E. Witten. Baryons in the 1/n(c) expansion. Nucl. Phys., B160:57, 1979.

[96] A. Zee. Quantum Field Theory in a Nutshell. Princeton University Press, 2003. 



\section{Danksagung}

An erster Stelle möchte ich mich bei meinem Doktorvater Matthias Lutz für die Betreuung in den letzten Jahren bedanken. Die teilweise sehr emotionalen, aber immer fruchtbaren Disskussionen und seine hohen Ansprüche an die Qualität in der Wissenschaft haben in mir das Bestreben nach der Korrektheit und Stränge einer physikalischen Darstellung geweckt und gestärkt. Ein besonderer Dank gilt ihm auch für seine Bereitschaft, bei Problemen auch außerhalb der "betrieblichen Arbeitszeiten" zur Seite zu stehen.

Desweiteren möchte ich mich bei Jochen Wambach bedanken, dessen Vorlesungen an der Universität in Darmstadt mein Interesse an theoretischer Physik bereits im frühen Studium geweckt haben und der mir die Möglichkeit gegeben hat, an der GSI zu promovieren.

Mein Dank gilt Julian Hofmann, Ashot Gasparyan und Evgenij Kolomeitsev für die vielen Diskussionen rund um die Arbeit. Julian Hofmann, meinem jahrelangen Zimmernachbarn danke ich auch für die zahlreichen Diskussionen ausserhalb der Physik.

Und schließlich möchte ich die angenehme und produktive Arbeitsatmosphere in der Theoriegruppe der GSI hervorheben und allen Kollegen dafür herzlich danken. 


\section{Lebenslauf}

\section{Alexander Semke}

geb. am 16.01.1977

in Nikopol (Ukraine)

unverheiratet, ein Kind

09.1984 - 06.1994 Gymnasium in Ordzhonikidze (Ukraine)

Abschluß: anerkannt als Mittlere Reife (Gesamtnote: sehr gut)

09.1994 - 12.1994 Studium der Physik an der Staatlichen Universität Dnipropetrovsk (Ukraine)

09.1996 - 06.1998 Staatliches Aufbaugymnasium in Alzey

Sonderlehrgang zum Erwerb der allgemeinen Hochschulreife für Spätaussiedler

Abschluß: Allgemeine Hochschulreife (Gesamtnote: sehr gut)

08.1999 - $06.2000 \quad$ Zivildienst am Institut für Arbeitswissenschaft der Technischen Universität Darmstadt

10.1998 - 09.2005 Studium der Physik an der Technischen Universität Darmstadt

Diplomarbeit bei der Gesellschaft für Schwerionenforschung zum Thema "Baryonmassen in der chiralen Störungstheorie" Abschluß: Dipl.-Physiker (Gesamtnote: sehr gut)

10.2005 - 01.2010 Wissenschaftlicher Mitarbeiter bei der Gesellschaft für Schwerionenforschung und Anfertigung der Dissertation 


\section{Eidestattliche Erklärung}

Ich versichere, dass ich die vorliegende Dissertation selbständig, ohne unerlaubte Hilfsmittel und nur unter Verwendung der in der Arbeit angegebenen Literatur angefertigt habe.

Die Arbeit wurde bisher in gleicher oder ähnlicher Form keiner anderen Prüfungsbehörde vorgelegt und auch nicht veröffentlicht.

Ort

Datum

Unterschrift 